\title{
Total Synthesis of Euonymine and Euonyminol Octaacetate
}

Yinghua Wang, Toshiya Nagai, Itsuki Watanabe, Koichi Hagiwara, and Masayuki Inoue*

Graduate School of Pharmaceutical Sciences

The University of Tokyo, Hongo, Bunkyo-ku, Tokyo 113-0013, Japan

Fax: $(+81) 3-5841-0568$

Email:inoue@mol.f.u-tokyo.ac.jp

Supporting Information

123 Pages

Contents:

1. Experimental Procedures

2. Comparison of ${ }^{1} \mathrm{H}$ and ${ }^{13} \mathrm{C}\left\{{ }^{1} \mathrm{H}\right\} \mathrm{NMR}$ data of natural, reported, and synthetic $\mathbf{1}$ and $2 \mathrm{~S} 40$

3. X-ray structures and crystallographic data of 56 and 63

4. Determination of enantiopurity of alcohol $\mathbf{1 3}$ and enone $\mathbf{1 8}$

5. Structural assignment of Diels-Alder adducts $\mathbf{1 5}$

6. Determination of the $\mathrm{C} 8$-configuration of enone $\mathbf{1 8}$

7. Investigation of C-ring construction

8. References

9. NMR charts 


\section{Experimental Procedures}

General methods: All reactions sensitive to air or moisture were carried out under argon atmosphere in dry solvents unless otherwise noted. $\mathrm{CH}_{2} \mathrm{Cl}_{2}, \mathrm{DMF}, \mathrm{Et}_{2} \mathrm{O}, \mathrm{THF}$, and toluene were purified by Glass Contour solvent dispensing system (Nikko Hansen \& Co., Ltd.). All other reagents were used as supplied unless otherwise noted. All reactions under heating conditions were performed in an oil bath. The microwave irradiation experiments were performed at Initiator System from Biotage Corporation using standard Pyrex vessels (capacity $20 \mathrm{~mL}$ ) sealed with a vial cap. The temperature profiles of the solvents (power control) were monitored using calibrated infrared temperature control mounted underneath the reaction vessel. Analytical thin-layer chromatography (TLC) was performed using E. Merck Silica gel $60 \mathrm{~F}_{254}$ pre-coated plates $(0.25 \mathrm{~mm})$. Preparative thin-layer chromatography (PTLC) was performed using E. Merck Silica gel $60 \mathrm{~F}_{254}$ pre-coated plates $(0.5 \mathrm{~mm})$. Flash column chromatography was performed using 40-50 $\mu \mathrm{m}$ Silica Gel 60N (Kanto Chemical Co., Inc.) or 32-53 $\mu \mathrm{m}$ Silica-gel BW-300 (Fuji Silysia Chemical Ltd.). Automated flash chromatography was conducted with a Yamazen Smart Flash EPCLC-AI-580S system using the prepacked silica gel columns. Unless otherwise noted, Silica-gel BW-300 was used for the purification. High-performance liquid chromatography (HPLC) was equipped with a JASCO HPLC system (pump: JASCO PU 2086 Plus x2, detector: JASCO MX-2080-32, degasser: ERC Inc. EC3325, data analysis by JASCO ChromNAV 1.5.2.). Melting points were measured on Yanaco MP-J3 micro melting point apparatus, and were uncorrected. Optical rotations were measured on JASCO P-2200 polarimeter at room temperature using sodium D line. Infrared (IR) spectra were recorded on JASCO FT/IR-4100 spectrometer as a thin film on $\mathrm{KBr}$ or $\mathrm{CaF}_{2} .{ }^{1} \mathrm{H}$ and ${ }^{13} \mathrm{C}\left\{{ }^{1} \mathrm{H}\right\}$ NMR spectra were recorded on JEOL JNM-ECS-400, JNM-ECX-500, or JNM-ECZ-500 spectrometer. Chemical shifts were reported in ppm on the $\delta$ scale relative to $\mathrm{CHCl}_{3}$ ( $\delta 7.26$ for ${ }^{1} \mathrm{H} \mathrm{NMR}$ ), $\mathrm{CDCl}_{3}$ ( $\delta 77.0$ for ${ }^{13} \mathrm{C}\left\{{ }^{1} \mathrm{H}\right\} \mathrm{NMR}$ ), $\mathrm{C}_{6} \mathrm{D}_{5} \mathrm{H}\left(\delta 7.16\right.$ for ${ }^{1} \mathrm{H}$ NMR), $\mathrm{C}_{6} \mathrm{D}_{6}$ ( $\delta 128.0$ for ${ }^{13} \mathrm{C}\left\{{ }^{1} \mathrm{H}\right\}$ NMR $), \mathrm{CD}_{2} \mathrm{HOD}\left(\delta 3.31\right.$ for ${ }^{1} \mathrm{H}$ NMR $), \mathrm{CD}_{3} \mathrm{OD}\left(\delta 49.0\right.$ for ${ }^{13} \mathrm{C}\left\{{ }^{1} \mathrm{H}\right\}$ $\mathrm{NMR}), \mathrm{CO}\left(\mathrm{CD}_{3}\right)\left(\mathrm{CD}_{2} \mathrm{H}\right)\left(\delta 2.05\right.$ for $\left.{ }^{1} \mathrm{H} \mathrm{NMR}\right)$, and $\mathrm{CO}\left(\mathrm{CD}_{3}\right)_{2}\left(\delta 206.3\right.$ for $\left.{ }^{13} \mathrm{C}\left\{{ }^{1} \mathrm{H}\right\} \mathrm{NMR}\right)$ as internal references. Signal patterns are indicated as s, singlet; d, doublet; t, triplet; q, quartet; m, multiplet; br, broaden peak. The carbon numbering of compounds corresponds to that of euonymine (1) and euonyminol octaacetate (2) unless otherwise noted (Figure S1). High resolution mass spectra were measured on JEOL JMS-T100LP (ESI-TOF) or BRUKER DALTONICS microTOF II (ESI-TOF). Xray crystallographic analysis was conducted using Inorganic Fine Crystal Structural Diffractometer VariMax Dual (Rigaku Co., Ltd.) with Mo radiation $(\lambda=0.71073 \AA$ ) . 


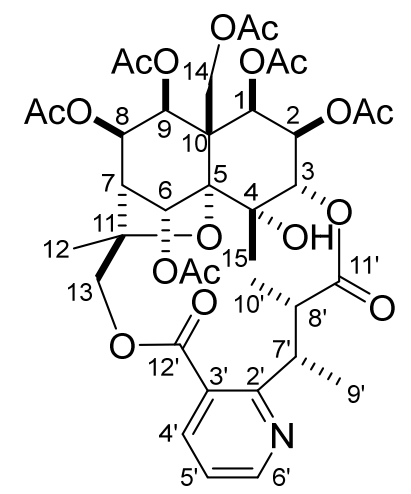

euonymine (1)

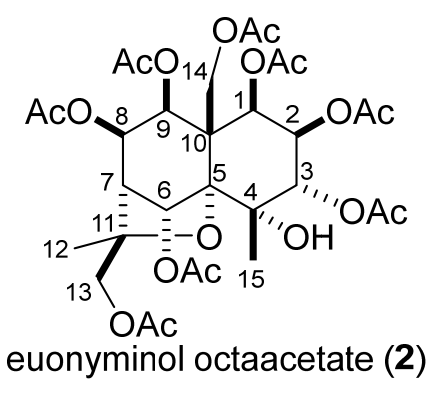

Figure S1. Numbering system of euonymine (1) and euonyminol octaacetate (2)

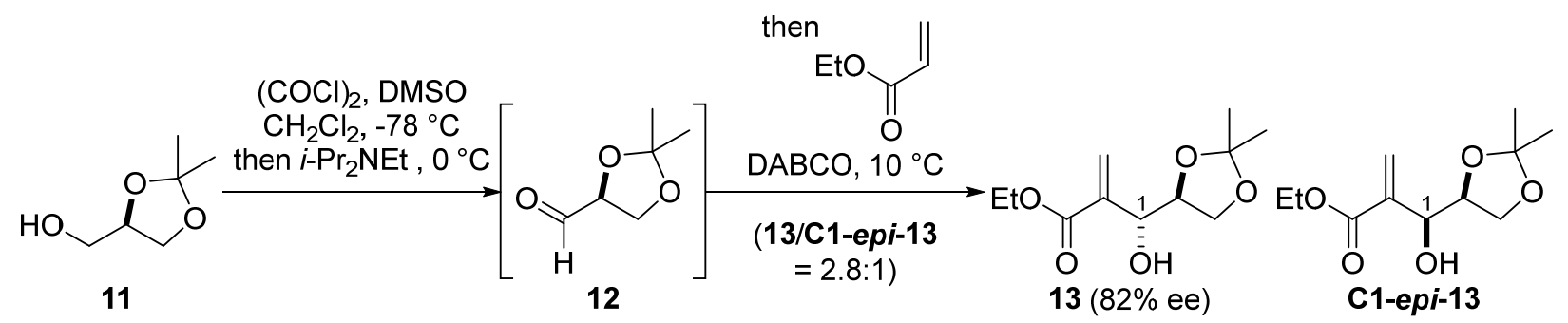

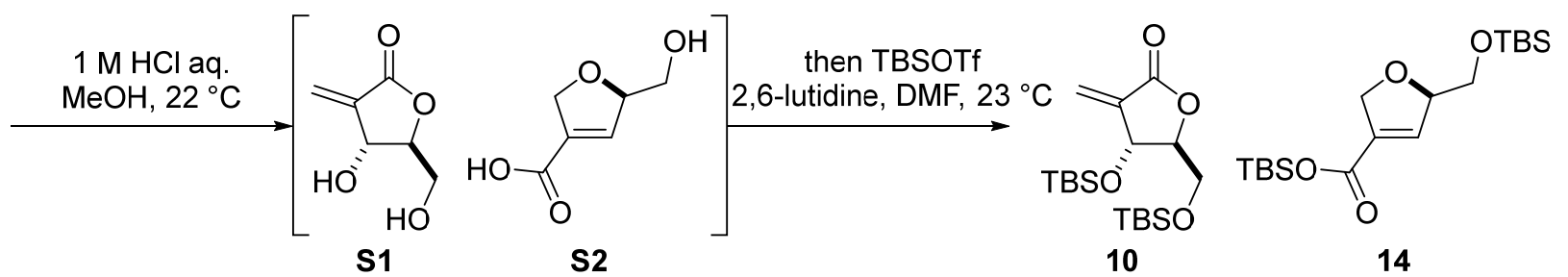

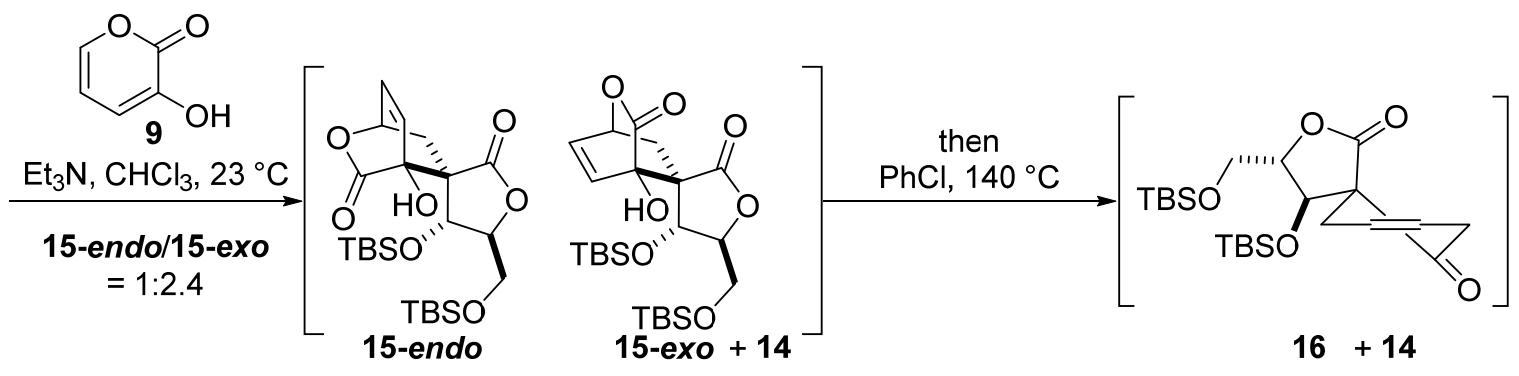

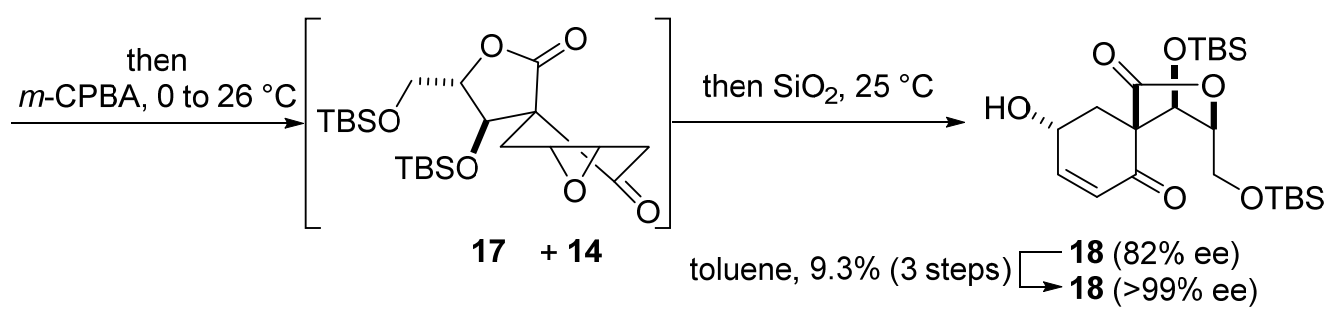

Enone 18 from $(\boldsymbol{R})$-glycerol acetonide (11). A solution of dimethyl sulfoxide (DMSO, $43.0 \mathrm{~mL}, 605$ mmol) in $\mathrm{CH}_{2} \mathrm{Cl}_{2}(70.0 \mathrm{~mL})$ at $-20{ }^{\circ} \mathrm{C}$ was added to a solution of $(\mathrm{COCl})_{2}(26.0 \mathrm{~mL}, 303 \mathrm{mmol})$ in $\mathrm{CH}_{2} \mathrm{Cl}_{2}(140 \mathrm{~mL})$ at $-66^{\circ} \mathrm{C}$ (internal temperature) over $50 \mathrm{~min}$. After the mixture was stirred at -78 ${ }^{\circ} \mathrm{C}$ for $20 \mathrm{~min},(R)$-glycerol acetonide (11) $(32.5 \mathrm{~g}, 246 \mathrm{mmol})$ in $\mathrm{CH}_{2} \mathrm{Cl}_{2}(70.0 \mathrm{~mL})$ at $-20{ }^{\circ} \mathrm{C}$ was added to the mixture at $-66{ }^{\circ} \mathrm{C}$ (internal temperature) over $20 \mathrm{~min}$. After the reaction mixture was 
stirred at $-78^{\circ} \mathrm{C}$ for $40 \mathrm{~min}, i$-Pr2 $\mathrm{NEt}(200 \mathrm{~mL}, 1.16 \mathrm{~mol})$ was added to the reaction mixture at $-78^{\circ} \mathrm{C}$ over $10 \mathrm{~min}$. After being stirred at $0{ }^{\circ} \mathrm{C}$ for $40 \mathrm{~min}$, the reaction mixture was concentrated $\left(10{ }^{\circ} \mathrm{C}, 121\right.$ $\mathrm{mmHg}$ ) to afford the crude aldehyde 12, which was used in the next reaction without further purification.

1,4-Diazabicyclo[2.2.2] octane (DABCO, $17.6 \mathrm{~g}, 157 \mathrm{mmol}$ ) was added to the above crude aldehyde 12 in ethyl acrylate $(80.0 \mathrm{~mL}, 751 \mathrm{mmol})$ at $0{ }^{\circ} \mathrm{C}$. After being stirred at $10{ }^{\circ} \mathrm{C}$ for $6 \mathrm{~d}$, the reaction mixture was passed through a pad of silica gel (Kanto, $750 \mathrm{~g}$, hexane/EtOAc $=10 / 1$ ) to afford the crude 2.8:1 mixture of $\mathbf{1 3}$ and C1-epi-13 (16.6 g, Page S59), which was used in the next reaction without further purification. To collect the analytical data, $\mathbf{1 3}$ and $\mathbf{C 1 - e p i - 1 3}$ were partially purified by flash column chromatography on silica gel (Kanto, hexane/EtOAc $=10 / 1)$. The stereochemistry of 13 was determined by converting 13 to dienophile 10 (Page S5). The enantiomeric excess was determined to be $82 \%$ by the chiral HPLC analysis [CHIRALCEL OD-H (Daicel), hexane/ $i-\mathrm{PrOH}=$ 98/2, $1.0 \mathrm{~mL} / \mathrm{min}, 220 \mathrm{~nm}, t_{\mathrm{R}} 8.42 \mathrm{~min}\left(\right.$ ent-13), $t_{\mathrm{R}} 9.39 \mathrm{~min}$ (13)] (Page S51):

13: colorless oil. $[\alpha]_{\mathrm{D}}{ }^{26}-2.75$ (c 1.10, $\mathrm{CHCl}_{3}$ ). IR (film): 3473, 2987, 2953, 2891, 1720, 1632, 1441, 1373, 1214, $1065 \mathrm{~cm}^{-1} .{ }^{1} \mathrm{H}$ NMR (500 MHz, CDCl $)$ : $\delta 6.35$ (1H, s, H9a), 5.97 (1H, s, H9b), $4.51(1 \mathrm{H}$, $\mathrm{d}, J=5.2 \mathrm{~Hz}, \mathrm{H} 1), 4.34(1 \mathrm{H}, \mathrm{ddd}, J=6.3,6.3,5.2 \mathrm{~Hz}, \mathrm{H} 2), 4.23\left(2 \mathrm{H}, \mathrm{q}, J=7.5 \mathrm{~Hz}, \mathrm{OCH}_{2} \mathrm{CH}_{3}\right), 3.95-$ $3.90(2 \mathrm{H}, \mathrm{m}, \mathrm{H} 3), 2.99(1 \mathrm{H}, \mathrm{br} \mathrm{s}, \mathrm{OH}), 1.43\left(3 \mathrm{H}, \mathrm{s}, \mathrm{CH}_{3}\right.$ of acetonide), 1.34 (3H, s, $\mathrm{CH}_{3}$ of acetonide), $1.31\left(3 \mathrm{H}, \mathrm{t}, J=7.5 \mathrm{~Hz}, \mathrm{OCH}_{2} \mathrm{CH}_{3}\right) .{ }^{13} \mathrm{C}\left\{{ }^{1} \mathrm{H}\right\} \mathrm{NMR}\left(125 \mathrm{MHz}, \mathrm{CDCl}_{3}\right): \delta 166.1,138.1,127.4,109.7$, 76.5, 71.2, 65.1, 61.0, 26.5, 25.0, 14.1. HRMS (ESI-TOF) $[\mathrm{M}+\mathrm{Na}]^{+} \mathrm{m} / \mathrm{z}$ : Calcd for $\mathrm{C}_{11} \mathrm{H}_{18} \mathrm{O}_{5} \mathrm{Na}$ 253.1052; Found 253.1041.

C1-epi-13: colorless oil. $[\alpha]_{\mathrm{D}}{ }^{23}+25.7$ ( $0.480, \mathrm{CHCl}_{3}$ ). IR (film): 3476, 2985, 2935, 1712, 1631, 1376, 1259, 1156, $1064 \mathrm{~cm}^{-1} .{ }^{1} \mathrm{H}$ NMR (400 MHz, $\left.\mathrm{CDCl}_{3}\right): \delta 6.38(1 \mathrm{H}, \mathrm{s}, \mathrm{H} 9 \mathrm{a}), 5.96(1 \mathrm{H}, \mathrm{d}, J=1.4$ Hz, H9b), 4.47 (1H, d, $J=4.1 \mathrm{~Hz}, \mathrm{H} 1), 4.30$ (1H, ddd, $J=7.8,6.9,4.1 \mathrm{~Hz}, \mathrm{H} 2), 4.23$ (2H, q, $J=7.3$ $\left.\mathrm{Hz}, \mathrm{OCH}_{2} \mathrm{CH}_{3}\right), 4.02(1 \mathrm{H}, \mathrm{dd}, J=8.7,7.8 \mathrm{~Hz}, \mathrm{H3a}), 3.87(1 \mathrm{H}, \mathrm{dd}, J=8.7,6.9 \mathrm{~Hz}, \mathrm{H} 3 \mathrm{~b}), 2.75(1 \mathrm{H}, \mathrm{br}$ s, $\mathrm{OH}), 1.46\left(3 \mathrm{H}, \mathrm{s}, \mathrm{CH}_{3}\right.$ of acetonide), $1.36\left(3 \mathrm{H}, \mathrm{s}, \mathrm{CH}_{3}\right.$ of acetonide), $1.31(3 \mathrm{H}, \mathrm{t}, J=7.3 \mathrm{~Hz}$, $\left.\mathrm{OCH}_{2} \mathrm{CH}_{3}\right) .{ }^{13} \mathrm{C}\left\{{ }^{1} \mathrm{H}\right\}$ NMR $\left(100 \mathrm{MHz}, \mathrm{CDCl}_{3}\right): \delta 166.0,139.5,126.9,109.8,77.6,70.8,66.3,61.0$, 26.4, 25.1, 14.1. HRMS (ESI-TOF) $[\mathrm{M}+\mathrm{Na}]^{+} \mathrm{m} / \mathrm{z}$ : Calcd for $\mathrm{C}_{11} \mathrm{H}_{18} \mathrm{O}_{5} \mathrm{Na} 253.1052$; Found 253.1062. $\mathrm{HCl}$ (1 $\mathrm{M}$ aqueous solution, $36.0 \mathrm{~mL}, 36.0 \mathrm{mmol}$ ) was added to a solution of the above crude mixture of 13 and C1-epi-13 (16.6 g) in $\mathrm{MeOH}(180 \mathrm{~mL})$. After being stirred at $22{ }^{\circ} \mathrm{C}$ for $18 \mathrm{~h}$, the reaction mixture was concentrated to afford the crude mixture of $\mathbf{S 1}$ and $\mathbf{S 2}$, which was used in the next reaction without further purification.

tert-Butyldimethylsilyl trilfluoromethanesulfonate (TBSOTf, $30.0 \mathrm{~mL}, 131 \mathrm{mmol}$ ) was added to a solution of the above crude mixture of $\mathbf{S 1}$ and $\mathbf{S 2}$, and 2,6-lutidine (31.0 mL, $268 \mathrm{mmol})$ in DMF (180 $\mathrm{mL})$ at $0{ }^{\circ} \mathrm{C}$. After the reaction mixture was stirred at $23{ }^{\circ} \mathrm{C}$ for $3.5 \mathrm{~h}$, saturated aqueous $\mathrm{NH}_{4} \mathrm{Cl}(100$ $\mathrm{mL}$ ) was added to the mixture at $0{ }^{\circ} \mathrm{C}$. The resultant mixture was extracted with $\mathrm{Et}_{2} \mathrm{O}(500 \mathrm{~mL}$ x3). 
The combined organic layers were dried over $\mathrm{Na}_{2} \mathrm{SO}_{4}$, filtered, and concentrated. The residue was passed through a pad of silica gel (Kanto, $400 \mathrm{~g}$, hexane/EtOAc $=20 / 1)$ to afford a crude mixture of dienophile 10 and 14 (14.2 g), which was used in the next reaction without further purification. To collect the analytical data, $\mathbf{1 0}$ and $\mathbf{1 4}$ were partially purified by flash column chromatography on silica gel (Kanto, hexane/EtOAc $=20 / 1)$. The stereochemistry of $\mathbf{1 0}$ was confirmed by comparing ${ }^{1} \mathrm{H}$ and ${ }^{13} \mathrm{C}\left\{{ }^{1} \mathrm{H}\right\}$ NMR data of $\mathbf{1 0}$ and the reported ent-10. ${ }^{\mathrm{S} 1}$

10: white solid. m.p. $68-69^{\circ} \mathrm{C}$. $[\alpha]_{\mathrm{D}}{ }^{26}-13.9$ (c 0.425, $\left.\mathrm{CHCl}_{3}\right)$. IR (film): 2955, 2929, 2858, 1780, 1715 , 1641, 1471, 1257, $1096 \mathrm{~cm}^{-1} .{ }^{1} \mathrm{H}$ NMR $\left(500 \mathrm{MHz}, \mathrm{CDCl}_{3}\right): \delta 6.34(1 \mathrm{H}, \mathrm{d}, J=2.3 \mathrm{~Hz}, \mathrm{H} 9 \mathrm{a}), 5.77(1 \mathrm{H}$, $\mathrm{d}, J=2.3 \mathrm{~Hz}, \mathrm{H} 9 \mathrm{~b}), 4.89$ (1H, ddd, $J=4.0,2.3,2.3 \mathrm{~Hz}, \mathrm{H} 1), 4.22(1 \mathrm{H}, \mathrm{ddd}, J=4.0,3.5,2.9 \mathrm{~Hz}, \mathrm{H} 2)$, $3.86(1 \mathrm{H}, \mathrm{dd}, J=12.0,3.5 \mathrm{~Hz}, \mathrm{H} 3 \mathrm{a}), 3.79(1 \mathrm{H}, \mathrm{dd}, J=12.0,2.9 \mathrm{~Hz}, \mathrm{H} 3 \mathrm{~b}), 0.91$ (9H, s, $t$-Bu of TBS), $0.86\left(9 \mathrm{H}, \mathrm{s}, t-\mathrm{Bu}\right.$ of TBS), $0.14\left(3 \mathrm{H}, \mathrm{s}, \mathrm{CH} 3\right.$ of TBS), $0.13\left(3 \mathrm{H}, \mathrm{s}, \mathrm{CH} 3\right.$ of TBS), 0.07 (3H, s, $\mathrm{CH}_{3}$ of TBS), 0.05 (3H, s, $\mathrm{CH}_{3}$ of TBS). ${ }^{13} \mathrm{C}\left\{{ }^{1} \mathrm{H}\right\} \mathrm{NMR}\left(125 \mathrm{MHz}, \mathrm{CDCl}_{3}\right): \delta 168.8,139.5,124.3,85.1,69.2$, 61.4, 25.7 (3C), 25.6 (3C), 18.2, 17.9, -4.4, -4.6, -5.5, -5.6. HRMS (ESI-TOF) $[\mathrm{M}+\mathrm{Na}]^{+} \mathrm{m} / \mathrm{z}$ : Calcd for $\mathrm{C}_{18} \mathrm{H}_{36} \mathrm{O}_{4} \mathrm{Si}_{2} \mathrm{Na} 395.2050$; Found 395.2064.

14: colorless oil. $[\alpha]_{\mathrm{D}}{ }^{26}+1.2\left(c 0.31, \mathrm{CHCl}_{3}\right)$. IR (film): 2956, 2930, 2858, 1715, 1472, 1388, 1306, 1259, 1176, $1096 \mathrm{~cm}^{-1} .{ }^{1} \mathrm{H}$ NMR (400 MHz, C6 $\left.\mathrm{D}_{6}\right): \delta 6.86(1 \mathrm{H}, \mathrm{s}, \mathrm{H} 1), 4.46-4.35$ (3H, m, H2, H9), $3.40-3.32$ (2H, m, H3), 0.93 (9H, s, $t$-Bu of TBS), 0.89 (9H, s, $t$-Bu of TBS), -0.02 (9H, s, CH3 of TBS $\mathrm{x} 3),-0.03\left(3 \mathrm{H}, \mathrm{s}, \mathrm{CH} 3\right.$ of TBS). ${ }^{13} \mathrm{C}\left\{{ }^{1} \mathrm{H}\right\}$ NMR $\left(125 \mathrm{MHz}, \mathrm{CDCl}_{3}\right): \delta 171.9,146.3,135.8,82.1,63.1$, 58.2, 25.8 (3C), 25.7 (3C), 18.23, 18.15, -5.5 (3C), -5.6. HRMS (ESI-TOF) M+Na $]^{+} \mathrm{m} / \mathrm{z}$ : Calcd for $\mathrm{C}_{18} \mathrm{H}_{36} \mathrm{O}_{4} \mathrm{Si}_{2} \mathrm{Na}$ 395.2050; Found 395.2047.

$\mathrm{Et}_{3} \mathrm{~N}(5.30 \mathrm{~mL}, 38.0 \mathrm{mmol})$ was added to a solution of the above crude mixture of dienophile $\mathbf{1 0}$ and 14 (14.2 g), and diene 9 (4.11 g, $36.7 \mathrm{mmol})$ in $\mathrm{CHCl}_{3}(70.0 \mathrm{~mL})$ at $23{ }^{\circ} \mathrm{C}$. After being stirred at 23 ${ }^{\circ} \mathrm{C}$ for $16 \mathrm{~h}$, the reaction mixture was concentrated to afford the crude mixture of $\mathbf{1 5}$-endo and 15-exo (15-endo/15-exo = 1:2.4, Page S64), and 14 (15.4 g), which was used in the next reaction without further purification. To collect the analytical data, 15-endo and 15-exo were partially purified by flash column chromatography on silica gel (Kanto, hexane/EtOAc $=20 / 1)$. The stereochemistries of 15-exo and 15-endo were determined as shown in Page S53.

15-endo: white amorphous. $[\alpha]_{\mathrm{D}}{ }^{24}-22.8\left(c 0.600, \mathrm{CHCl}_{3}\right)$. IR (film): 3454, 2933, 2858, 1760, 1467 , 1360, 1255, 1133, 837, $779 \mathrm{~cm}^{-1} .{ }^{1} \mathrm{H} \mathrm{NMR}\left(400 \mathrm{MHz}, \mathrm{CDCl}_{3}\right): \delta 6.60(1 \mathrm{H}, \mathrm{dd}, J=7.8,1.8 \mathrm{~Hz}, \mathrm{H} 6)$, $6.53(1 \mathrm{H}, \mathrm{dd}, J=7.8,5.0 \mathrm{~Hz}, \mathrm{H} 7), 5.33(1 \mathrm{H}, \mathrm{dddd}, J=5.0,4.6,1.8,1.4 \mathrm{~Hz}, \mathrm{H} 8), 4.73(1 \mathrm{H}, \mathrm{d}, J=6.4$ Hz, H1), 4.05 (1H, ddd, $J=6.4,3.6,2.3 \mathrm{~Hz}, \mathrm{H} 2), 3.99$ (1H, dd, $J=11.9,2.3 \mathrm{~Hz}, \mathrm{H} 3 \mathrm{a}), 3.89$ (1H, dd, $J=11.9,3.6 \mathrm{~Hz}, \mathrm{H} 3 \mathrm{~b}), 3.83(1 \mathrm{H}, \mathrm{s}, \mathrm{OH}), 3.17$ (1H, dd, $J=13.3,4.6 \mathrm{~Hz}, \mathrm{H} 9 \mathrm{a}), 1.80(1 \mathrm{H}, \mathrm{dd}, J=13.3$, $1.4 \mathrm{~Hz}, \mathrm{H} 9 \mathrm{~b}), 0.92$ (9H, s, $t$-Bu of TBS), 0.90 (9H, s, $t$-Bu of TBS), 0.133 (3H, s, CH3 of TBS), 0.126 (3H, s, $\mathrm{CH}_{3}$ of TBS), 0.102 (3H, s, $\mathrm{CH}_{3}$ of TBS), 0.096 (3H, s, $\mathrm{CH}_{3}$ of TBS). ${ }^{13} \mathrm{C}\left\{{ }^{1} \mathrm{H}\right\} \mathrm{NMR}(100 \mathrm{MHz}$, $\left.\mathrm{CDCl}_{3}\right): \delta 174.5,172.9,136.9,130.0,84.5,76.1,74.0,67.5,60.8,51.9,32.5,25.9$ (3C), $25.8(3 \mathrm{C})$, 
18.3, 18.1, -4.1, -5.2, -5.3, -5.5. HRMS (ESI-TOF) $[\mathrm{M}+\mathrm{Na}]^{+} \mathrm{m} / \mathrm{z}$ : Calcd for $\mathrm{C}_{23} \mathrm{H}_{40} \mathrm{O}_{7} \mathrm{Si}_{2} \mathrm{Na}$ 507.2205; Found 507.2205.

15-exo: white solid. m.p. $135-138{ }^{\circ} \mathrm{C}$. $[\alpha]_{\mathrm{D}}{ }^{27}-27.7$ (c 0.700, $\mathrm{CHCl}_{3}$ ). IR (film): 3493, 2934, 2858, 1778, 1466, 1332, 1255, 1138, 837, $778 \mathrm{~cm}^{-1} .{ }^{1} \mathrm{H}$ NMR (400 MHz, $\left.\mathrm{CDCl}_{3}\right): \delta 6.50(1 \mathrm{H}, \mathrm{dd}, J=7.8$, $5.0 \mathrm{~Hz}, \mathrm{H} 7), 6.34$ (1H, dd, $J=7.8,1.8 \mathrm{~Hz}, \mathrm{H} 6), 5.34$ (1H, dddd, $J=5.0,3.6,1.8,1.4$ Hz, H8), 4.74 $(1 \mathrm{H}, \mathrm{d}, J=5.5 \mathrm{~Hz}, \mathrm{H} 1), 4.07$ (1H, ddd, $J=5.5,4.6,4.1 \mathrm{~Hz}, \mathrm{H} 2), 3.98$ (1H, dd, $J=11.9,4.1 \mathrm{~Hz}, \mathrm{H} 3 \mathrm{a})$, $3.847(1 \mathrm{H}, \mathrm{dd}, J=11.9,4.6 \mathrm{~Hz}, \mathrm{H} 3 \mathrm{~b}), 3.846(1 \mathrm{H}, \mathrm{s}, \mathrm{OH}), 2.68(1 \mathrm{H}, \mathrm{dd}, J=13.7,1.4 \mathrm{~Hz}, \mathrm{H} 9 \mathrm{a}), 2.35$ $(1 \mathrm{H}, \mathrm{dd}, J=13.7,3.6 \mathrm{~Hz}, \mathrm{H} 9 \mathrm{~b}), 0.91$ (9H, s, $t$-Bu of TBS), $0.86(9 \mathrm{H}, \mathrm{s}, t$-Bu of TBS), 0.15 (3H, s, CH3 of TBS), $0.09\left(6 \mathrm{H}, \mathrm{s}, \mathrm{CH}_{3}\right.$ of TBS x2), $0.08\left(3 \mathrm{H}, \mathrm{s}, \mathrm{CH}\right.$ of TBS). ${ }^{13} \mathrm{C}\left\{{ }^{1} \mathrm{H}\right\} \mathrm{NMR}\left(100 \mathrm{MHz}, \mathrm{CDCl}_{3}\right)$ : $\delta 176.2,173.1,135.3,132.5,85.2,76.4,72.9,69.0,60.8,53.0,32.5,25.80$ (3C), 25.75 (3C), 18.3, 17.9, $-4.1,-4.4,-5.35,-5.42$. HRMS (ESI-TOF) $[\mathrm{M}+\mathrm{Na}]^{+} \mathrm{m} / \mathrm{z}$ : Calcd for $\mathrm{C}_{23} \mathrm{H}_{40} \mathrm{O}_{7} \mathrm{Si}_{2} \mathrm{Na}$ 507.2205; Found 507.2183.

Argon was bubbled through a solution of the above crude 15-endo, 15-exo, and 14 (15.4 g) in $\mathrm{PhCl}$ $\left(49.0 \mathrm{~mL}\right.$ ) at $26^{\circ} \mathrm{C}$ for $30 \mathrm{~min}$ to remove the residual $\mathrm{O}_{2}$. After the reaction mixture was stirred at 140 ${ }^{\circ} \mathrm{C}$ for $3 \mathrm{~h}$ under argon flow, the reaction mixture was cooled to $0{ }^{\circ} \mathrm{C}$ and 3-chloroperoxybenzoic acid ( $m$-CPBA, 77\% purity, $11.8 \mathrm{~g}, 52.7 \mathrm{mmol}$ ) was added to the reaction mixture. After the reaction mixture was stirred at $26^{\circ} \mathrm{C}$ for $18 \mathrm{~h}$, saturated aqueous $\mathrm{NaHCO}_{3}(200 \mathrm{~mL})$ and saturated aqueous $\mathrm{Na}_{2} \mathrm{~S}_{2} \mathrm{O}_{3}(200 \mathrm{~mL})$ were successively added to the mixture at $0{ }^{\circ} \mathrm{C}$. The resultant mixture was extracted with $\mathrm{CH}_{2} \mathrm{Cl}_{2}(500 \mathrm{~mL} \times 3)$. The combined organic layers were dried over $\mathrm{Na}_{2} \mathrm{SO}_{4}$, filtered, and concentrated. The residue was treated with silica gel (Kanto, $500 \mathrm{~g}$ ) at $25{ }^{\circ} \mathrm{C}$ for $13 \mathrm{~h}$ and eluted with hexane/EtOAc (4/1 to 0/1). Concentration of the eluents afforded the crude enone 18 (14.2 g, 82\% ee, Page S52). Recrystallization from toluene (12 mL) afforded enone 18 (10.4 g, 22.8 mmol) in 9.3\% yield over 3 steps. The enantiomeric excess was determined to be $>99 \%$ by the chiral HPLC analysis [CHIRALPAK AS-RH (Daicel), $\mathrm{MeCN} / \mathrm{H}_{2} \mathrm{O}=45 / 55,1.0 \mathrm{~mL} / \mathrm{min}, 254 \mathrm{~nm}, t_{\mathrm{R}} 17.75 \mathrm{~min}(\mathbf{1 8}), t_{\mathrm{R}}$ $21.93 \min ($ ent-18)] (Page S52). The C8-stereochemistry of 18 was determined by the modified Mosher method (Page S55): white crystal. m.p. $127-128^{\circ} \mathrm{C} .[\alpha]_{\mathrm{D}}{ }^{23}+1.87$ (c 1.03, $\mathrm{CHCl}_{3}$ ). IR (film): 3464, 2954, 2931, 2887, 2859, 1776, 1684, 1256, 1142, $839 \mathrm{~cm}^{-1} .{ }^{1} \mathrm{H}$ NMR (400 MHz, $\left.\mathrm{CDCl}_{3}\right): \delta 7.07$ $(1 \mathrm{H}, \mathrm{ddd}, J=9.6,1.8,1.8 \mathrm{~Hz}, \mathrm{H} 7), 6.14(1 \mathrm{H}, \mathrm{dd}, J=9.6,2.3 \mathrm{~Hz}, \mathrm{H} 6), 5.38(1 \mathrm{H}, \mathrm{d}, J=7.8 \mathrm{~Hz}, \mathrm{H} 1)$, 4.98 (1H, m, H8), 4.17 (1H, ddd, $J=7.8,3.2,1.8 \mathrm{~Hz}, \mathrm{H} 2), 4.01$ (1H, dd, $J=12.4,1.8 \mathrm{~Hz}, \mathrm{H} 3 \mathrm{a}), 3.80$ (1H, dd, $J=12.4,3.2 \mathrm{~Hz}, \mathrm{H} 3 \mathrm{~b}), 2.42$ (1H, ddd, $J=13.7,5.0,1.8 \mathrm{~Hz}, \mathrm{H} 9 \mathrm{a}), 2.18$ (1H, dd, $J=13.7$, $11.0 \mathrm{~Hz}, \mathrm{H} 9 \mathrm{~b}), 1.97(1 \mathrm{H}, \mathrm{d}, J=6.4 \mathrm{~Hz}, \mathrm{OH}), 0.91$ (9H, s, $t$-Bu of TBS), 0.86 (9H, s, $t$-Bu of TBS), 0.12 (3H, s, $\mathrm{CH}_{3}$ of TBS), 0.09 (3H, s, $\mathrm{CH}_{3}$ of TBS), 0.07 (3H, s, $\mathrm{CH}_{3}$ of TBS), -0.03 (3H, s, $\mathrm{CH}_{3}$ of TBS). ${ }^{13} \mathrm{C}\left\{{ }^{1} \mathrm{H}\right\}$ NMR (100 MHz, $\left.\mathrm{CDCl}_{3}\right): \delta$ 192.0, 171.7, 154.8, 128.2, 83.3, 68.6, 64.5, 60.1, 58.4, 33.1, 25.7 (3C), 25.6 (3C), 18.2, 17.8, -4.5, -5.3, -5.4, -5.5. HRMS (ESI-TOF) $[\mathrm{M}+\mathrm{Na}]^{+} \mathrm{m} / \mathrm{z}$ : Calcd for $\mathrm{C}_{22} \mathrm{H}_{40} \mathrm{O}_{6} \mathrm{Si}_{2} \mathrm{Na} 479.2256$; Found 479.2238. 

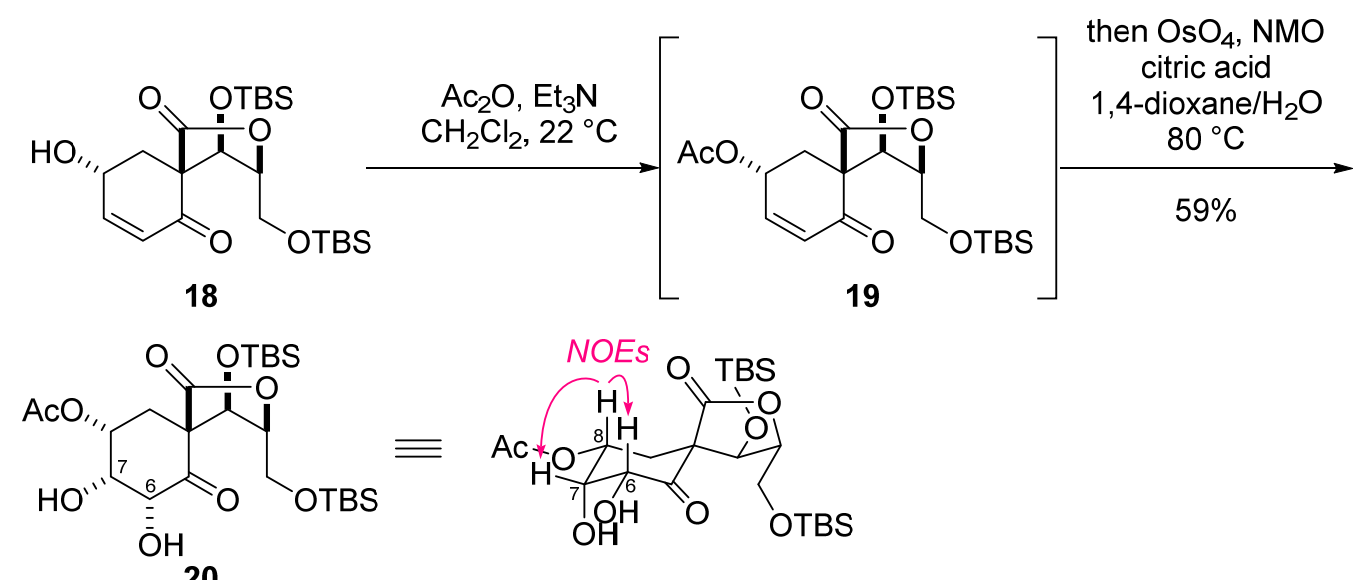

20

Diol 20. $\mathrm{Ac}_{2} \mathrm{O}(6.00 \mathrm{~mL}, 63.5 \mathrm{mmol})$ was added to a solution of enone 18 (9.72 g, $\left.21.3 \mathrm{mmol}\right)$ and $\mathrm{Et}_{3} \mathrm{~N}(8.90 \mathrm{~mL}, 64.0 \mathrm{mmol})$ in $\mathrm{CH}_{2} \mathrm{Cl}_{2}(210 \mathrm{~mL})$ at $22{ }^{\circ} \mathrm{C}$. After being stirred at $22{ }^{\circ} \mathrm{C}$ for $14 \mathrm{~h}$, the reaction mixture was concentrated to afford the crude enone $19(11.3 \mathrm{~g})$, which was used in the next reaction without further purification. To collect the analytical data, $\mathbf{1 9}$ was partially purified by flash column chromatography on silica gel (Kanto, hexane/EtOAc $=5 / 1)$ : white solid. m.p. $116-120{ }^{\circ} \mathrm{C}$. $[\alpha]_{\mathrm{D}^{21}}-19.9$ (c 1.01, $\mathrm{CHCl}_{3}$ ). IR (film): 2930, 2858, 1778, 1747, 1683, 1231, 1150, 1060, 837, $779 \mathrm{~cm}^{-}$ ${ }^{1} .{ }^{1} \mathrm{H}$ NMR $\left(400 \mathrm{MHz}, \mathrm{CDCl}_{3}\right): \delta 6.96(1 \mathrm{H}, \mathrm{ddd}, J=10.5,2.3,1.8 \mathrm{~Hz}, \mathrm{H} 7), 6.21(1 \mathrm{H}, \mathrm{dd}, J=10.5,2.3$ Hz, H6), 5.92 (1H, dddd, $J=11.0,5.5,2.3,1.8 \mathrm{~Hz}, \mathrm{H} 8), 5.42(1 \mathrm{H}, \mathrm{d}, J=8.2 \mathrm{~Hz}, \mathrm{H} 1), 4.14(1 \mathrm{H}$, ddd, $J=8.2,3.2,1.8 \mathrm{~Hz}, \mathrm{H} 2), 4.01(1 \mathrm{H}, \mathrm{dd}, J=12.4,1.8 \mathrm{~Hz}, \mathrm{H} 3 \mathrm{a}), 3.79(1 \mathrm{H}, \mathrm{dd}, J=12.4,3.2 \mathrm{~Hz}, \mathrm{H} 3 \mathrm{~b})$, $2.48(1 \mathrm{H}, \mathrm{ddd}, J=13.7,5.5,2.3 \mathrm{~Hz}, \mathrm{H} 9 \mathrm{a}), 2.21(1 \mathrm{H}, \mathrm{dd}, J=13.7,11.0 \mathrm{~Hz}, \mathrm{H} 9 \mathrm{~b}), 2.13\left(3 \mathrm{H}, \mathrm{s}, \mathrm{CH}_{3}\right.$ of Ac), 0.91 (9H, s, $t$-Bu of TBS), 0.86 (9H, s, $t$-Bu of TBS), $0.12\left(3 \mathrm{H}, \mathrm{s}, \mathrm{CH}_{3}\right.$ of TBS), 0.08 (3H, s, $\mathrm{CH}_{3}$ of TBS), $0.06\left(3 \mathrm{H}, \mathrm{s}, \mathrm{CH}_{3}\right.$ of TBS), $-0.03\left(3 \mathrm{H}, \mathrm{s}, \mathrm{CH} 3\right.$ of TBS). ${ }^{13} \mathrm{C}\left\{{ }^{1} \mathrm{H}\right\} \mathrm{NMR}\left(100 \mathrm{MHz}, \mathrm{CDCl}_{3}\right)$ : $\delta 191.5,171.1,169.9,150.4,129.5,83.1,68.4,67.2,59.9,58.2,29.7,25.7$ (3C), 25.6 (3C), 21.0, 18.2, 17.8, -4.6, -5.3, -5.4, -5.5. HRMS (ESI-TOF) $[\mathrm{M}+\mathrm{Na}]^{+} \mathrm{m} / \mathrm{z}$ : Calcd for $\mathrm{C}_{24} \mathrm{H}_{42} \mathrm{O}_{7} \mathrm{Si}_{2} \mathrm{Na} 521.2361$; Found 521.2360.

Citric acid (12.4 g, $64.6 \mathrm{mmol}$ ), $N$-methylmorpholine $N$-oxide (NMO, $7.52 \mathrm{~g}, 64.3 \mathrm{mmol}$ ), and $\mathrm{OsO}_{4}$ (0.16 M aqueous solution, $13.0 \mathrm{~mL}, 2.08 \mathrm{mmol}$ ) were successively added to a solution of the above crude enone $19(11.3 \mathrm{~g})$ in 1,4-dioxane $(110 \mathrm{~mL})$ and $\mathrm{H}_{2} \mathrm{O}(110 \mathrm{~mL})$ at $22{ }^{\circ} \mathrm{C}$. After the reaction mixture was stirred at $80{ }^{\circ} \mathrm{C}$ for $6.5 \mathrm{~h}$, saturated aqueous $\mathrm{NaHCO}_{3}(80 \mathrm{~mL})$ and saturated aqueous $\mathrm{Na}_{2} \mathrm{~S}_{2} \mathrm{O}_{3}(80 \mathrm{~mL})$ were successively added to the mixture at $22{ }^{\circ} \mathrm{C}$. The resultant mixture was extracted with EtOAc $(300 \mathrm{~mL} \times 3)$. The combined organic layers were dried over $\mathrm{Na}_{2} \mathrm{SO}_{4}$, filtered, and concentrated. The residue was purified by flash column chromatography on silica gel (Kanto, $200 \mathrm{~g}$, hexane/EtOAc $=5 / 1$ to 3/1) to afford diol $20(6.69 \mathrm{~g}, 12.6 \mathrm{mmol})$ in 59\% yield. The C6,7stereochemistries of $\mathbf{2 0}$ were determined by the NOE experiment (Page S71): pale yellow amorphous. $[\alpha]_{\mathrm{D}}^{30}-58.7\left(c 1.06, \mathrm{CHCl}_{3}\right)$. IR (film): 3481, 2930, 2858, 1771, 1730, 1254, 1146, 922, 838, $781 \mathrm{~cm}^{-}$ ${ }^{1} .{ }^{1} \mathrm{H}$ NMR (400 MHz, $\left.\mathrm{CDCl}_{3}\right): \delta 5.63(1 \mathrm{H}, \mathrm{m}, \mathrm{H} 8), 5.38(1 \mathrm{H}, \mathrm{d}, J=8.2 \mathrm{~Hz}, \mathrm{H} 1), 5.01(1 \mathrm{H}, \mathrm{d}, J=1.8$ 
Hz, H6), 4.53 (1H, br s, H7), $4.13(1 \mathrm{H}, \mathrm{d}, J=8.2 \mathrm{~Hz}, \mathrm{H} 2), 3.98(1 \mathrm{H}, \mathrm{d}, J=12.4 \mathrm{~Hz}, \mathrm{H} 3 \mathrm{a}), 3.78(1 \mathrm{H}$, dd, $J=12.4,2.8 \mathrm{~Hz}, \mathrm{H} 3 \mathrm{~b}), 3.57(1 \mathrm{H}$, br s, OH), $2.64(1 \mathrm{H}, \mathrm{dd}, J=13.3,12.8 \mathrm{~Hz}, \mathrm{H} 9 \mathrm{a}), 2.61$ (1H, br s, $\mathrm{OH}), 2.13\left(3 \mathrm{H}, \mathrm{s}, \mathrm{CH}_{3}\right.$ of Ac), $2.11(1 \mathrm{H}, \mathrm{m}, \mathrm{H} 9 \mathrm{~b}), 0.86(18 \mathrm{H}, \mathrm{s}, t$-Bu of TBS x2), 0.17 (3H, s, CH3 of TBS), 0.07 (3H, s, CH3 of TBS), $0.03\left(6 \mathrm{H}, \mathrm{s}, \mathrm{CH} 3\right.$ of TBS x2). ${ }^{13} \mathrm{C}\left\{{ }^{1} \mathrm{H}\right\} \mathrm{NMR}\left(100 \mathrm{MHz}, \mathrm{CDCl}_{3}\right): \delta$ 202.8, 171.3, 170.0, 83.5, 75.2, 73.1, 67.7, 66.8, 59.6, 59.0, 26.5, 25.7 (3C), 25.6 (3C), 21.1, 18.1, 17.9, $-4.6,-5.3,-5.47,-5.54$. HRMS (ESI-TOF) $[\mathrm{M}+\mathrm{Na}]^{+} \mathrm{m} / \mathrm{z}$ : Calcd for $\mathrm{C}_{24} \mathrm{H}_{44} \mathrm{O}_{9} \mathrm{Si}_{2} \mathrm{Na} 555.2416$; Found 555.2404 .
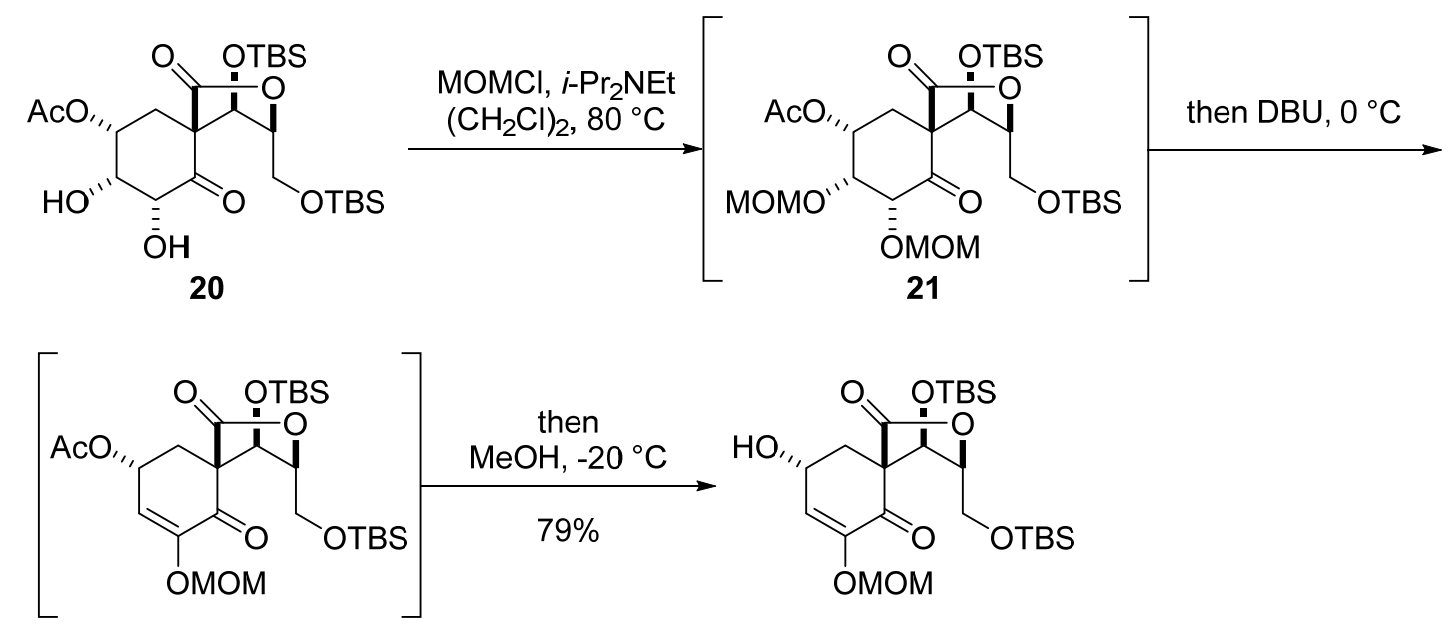

22

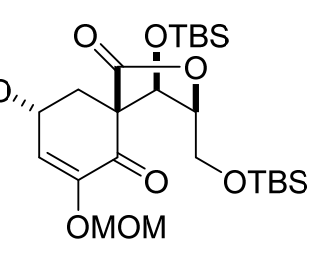

8

Enone 8. Chloromethyl methyl ether (MOMCl, $5.60 \mathrm{~mL}, 74.4 \mathrm{mmol}$ ) was added to a solution of diol 20 (5.65 g, $10.6 \mathrm{mmol})$ and $i$ - $\operatorname{Pr}_{2} \mathrm{NEt}(18.5 \mathrm{~mL}, 106 \mathrm{mmol})$ in 1,2-dichloroethane $(53.0 \mathrm{~mL})$ at $23{ }^{\circ} \mathrm{C}$. After the reaction mixture was stirred at $80{ }^{\circ} \mathrm{C}$ for $10 \mathrm{~h}, 1,8$-diazabicyclo[5.4.0]undec-7-ene (DBU, $16.0 \mathrm{~mL}, 107 \mathrm{mmol}$ ) was added to the mixture at $0{ }^{\circ} \mathrm{C}$. After the reaction mixture was stirred at $0{ }^{\circ} \mathrm{C}$ for $12 \mathrm{~h}, \mathrm{MeOH}(53.0 \mathrm{~mL})$ was added to the mixture at $-20^{\circ} \mathrm{C}$. After the reaction mixture was stirred at $-20{ }^{\circ} \mathrm{C}$ for $3.5 \mathrm{~h}$, saturated aqueous $\mathrm{NH}_{4} \mathrm{Cl}(100 \mathrm{~mL})$ was added to the mixture. After $\mathrm{H}_{2} \mathrm{O}(40 \mathrm{~mL})$ was added to the mixture, the resultant mixture was extracted with $\mathrm{CH}_{2} \mathrm{Cl}_{2}(150 \mathrm{~mL}$ x3). The combined organic layers were dried over $\mathrm{Na}_{2} \mathrm{SO}_{4}$, filtered, and concentrated. The residue was purified by flash column chromatography on silica gel (Kanto, $130 \mathrm{~g}$, hexane/EtOAc $=5 / 1$ to 2/1) to afford enone 8 (4.33 g, $8.39 \mathrm{mmol})$ in $79 \%$ yield: yellow amorphous. $[\alpha]_{\mathrm{D}}{ }^{18}-0.265$ (c 2.30, $\left.\mathrm{CHCl}_{3}\right)$. IR (film): 3503 , 2930, 2858, 1779, 1692, 1628, 1472, 1256, 1156, $1009 \mathrm{~cm}^{-1} .{ }^{1} \mathrm{H}$ NMR $\left(400 \mathrm{MHz}, \mathrm{CDCl}_{3}\right): \delta 6.43(1 \mathrm{H}$, $\mathrm{dd}, J=2.3,1.8 \mathrm{~Hz}, \mathrm{H} 7), 5.45(1 \mathrm{H}, \mathrm{d}, J=8.2 \mathrm{~Hz}, \mathrm{H} 1), 5.10\left(1 \mathrm{H}, \mathrm{d}, J=6.9 \mathrm{~Hz}, \mathrm{OCHaHbOCH}_{3}\right), 5.06$ $\left(1 \mathrm{H}, \mathrm{d}, J=6.9 \mathrm{~Hz}, \mathrm{OCHaHbOCH}_{3}\right), 4.98(1 \mathrm{H}, \mathrm{dddd}, J=10.5,6.9,5.5,2.3 \mathrm{~Hz}, \mathrm{H} 8), 4.16(1 \mathrm{H}, \mathrm{ddd}, J$ $=8.2,3.6,1.8 \mathrm{~Hz}, \mathrm{H} 2), 4.02(1 \mathrm{H}, \mathrm{dd}, J=12.4,1.8 \mathrm{~Hz}, \mathrm{H} 3 \mathrm{a}), 3.81(1 \mathrm{H}, \mathrm{dd}, J=12.4,3.6 \mathrm{~Hz}, \mathrm{H} 3 \mathrm{~b})$, $3.43\left(3 \mathrm{H}, \mathrm{s}, \mathrm{OCH}_{2} \mathrm{OCH}_{3}\right), 2.40(1 \mathrm{H}, \mathrm{ddd}, J=13.7,5.5,1.8 \mathrm{~Hz}, \mathrm{H} 9 \mathrm{a}), 2.14(1 \mathrm{H}, \mathrm{dd}, J=13.7,10.5 \mathrm{~Hz}$, H9b), 2.09 (1H, d, $J=6.9 \mathrm{~Hz}, \mathrm{OH}), 0.90$ (9H, s, $t$-Bu of TBS), 0.86 (9H, s, $t$-Bu of TBS), 0.12 (3H, s, $\mathrm{CH}_{3}$ of TBS), 0.08 (3H, s, $\mathrm{CH}_{3}$ of TBS), 0.06 (3H, s, $\mathrm{CH}_{3}$ of TBS), -0.03 (3H, s, $\mathrm{CH}_{3}$ of TBS). ${ }^{13} \mathrm{C}\left\{{ }^{1} \mathrm{H}\right\}$ 
NMR (100 MHz, $\left.\mathrm{CDCl}_{3}\right): \delta$ 187.6, 171.2, 147.7, 127.2, 94.3, 83.1, 68.8, 63.6, 59.8, 58.5, 56.3, 33.0, 25.7 (3C), 25.6 (3C), 18.1, 17.8, -4.6, -5.3, -5.4, -5.5. HRMS (ESI-TOF) $[\mathrm{M}+\mathrm{Na}]^{+} \mathrm{m} / \mathrm{z}$ : Calcd for $\mathrm{C}_{24} \mathrm{H}_{44} \mathrm{O}_{8} \mathrm{Si}_{2} \mathrm{Na} 539.2467$; Found 539.2462.

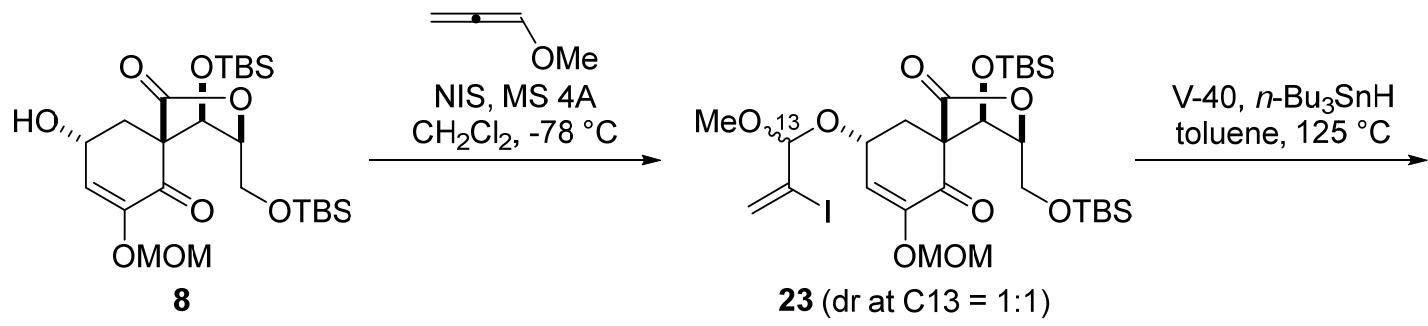

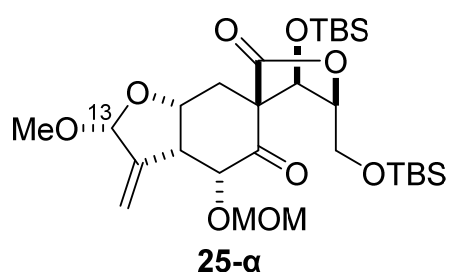

$29 \%$ (2 steps)

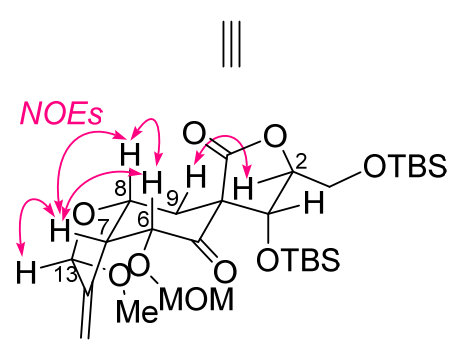

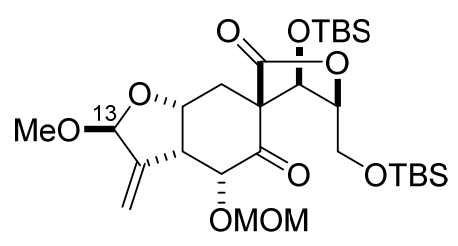

25- $\beta$

$30 \%$ (2 steps)

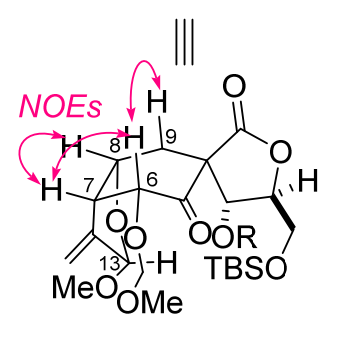

$\mathrm{R}=\mathrm{TBS}$

Acetal 25- $\alpha$ and 25- $\beta$. $N$-iodosuccinimide (NIS, recrystallized from $\mathrm{CCl}_{4}$ and 1,4-dioxane, ${ }^{\mathrm{S} 2} 4.92 \mathrm{~g}$, $21.9 \mathrm{mmol})$ and methoxyallene $(1.90 \mathrm{~mL}, 22.5 \mathrm{mmol})$ were successively added to a suspension of alcohol 8 (3.73 g, $7.23 \mathrm{mmol})$ and molecular sieves 4A (MS 4A, $5.72 \mathrm{~g}$ ) in $\mathrm{CH}_{2} \mathrm{Cl}_{2}(72.0 \mathrm{~mL})$ at $78{ }^{\circ} \mathrm{C}$. After the reaction mixture was stirred at $-78{ }^{\circ} \mathrm{C}$ for $2 \mathrm{~h}$, NIS $(1.68 \mathrm{~g}, 7.47 \mathrm{mmol})$ and methoxyallene $(620 \mu \mathrm{L}, 7.35 \mathrm{mmol})$ were successively added to the mixture at $-78{ }^{\circ} \mathrm{C}$. After the reaction mixture was stirred at $-78{ }^{\circ} \mathrm{C}$ for $1 \mathrm{~h}$, NIS $(1.58 \mathrm{~g}, 7.02 \mathrm{mmol})$ and methoxyallene $(600 \mu \mathrm{L}$, $7.11 \mathrm{mmol}$ ) were successively added to the mixture at $-78^{\circ} \mathrm{C}$. After the reaction mixture was stirred at $-78^{\circ} \mathrm{C}$ for $1 \mathrm{~h}, 5 \mathrm{wt} \%$ aqueous $\mathrm{KOH}(100 \mathrm{~mL})$ was added to the mixture. The resultant mixture was extracted with $\mathrm{CH}_{2} \mathrm{Cl}_{2}(100 \mathrm{~mL} \times 3)$. The combined organic layers were dried over $\mathrm{Na}_{2} \mathrm{SO}_{4}$ and filtered. After toluene $(100 \mathrm{~mL})$ was added to the filtrate, the solution was concentrated to remove $\mathrm{CH}_{2} \mathrm{Cl}_{2}$. The resultant toluene solution was passed through a pad of silica gel $(200 \mathrm{~g}$, hexane/EtOAc $=15 / 1$ to 9/1) to afford the crude vinyl iodide 23 (4.18 g, 1:1 diastereomeric mixture at C13, Page S73), which was used in the next reaction without further purification: ${ }^{1} \mathrm{H} \mathrm{NMR}\left(400 \mathrm{MHz}, \mathrm{CDCl}_{3}\right): \delta 6.63(1 \mathrm{H}$ x1/2, dd, $J=2.3,0.9 \mathrm{~Hz}, \mathrm{H} 12 \mathrm{a}), 6.62(1 \mathrm{H} \mathrm{x} 1 / 2, \mathrm{dd}, J=2.3,0.9 \mathrm{~Hz}, \mathrm{H} 12 \mathrm{a}), 6.52(1 \mathrm{H} \mathrm{x} 1 / 2, \mathrm{dd}, J=2.3$, $1.8 \mathrm{~Hz}, \mathrm{H} 7), 6.45$ (1H x1/2, dd, $J=2.3,1.8 \mathrm{~Hz}, \mathrm{H} 7), 6.13(1 \mathrm{H} \mathrm{x1} / 2, \mathrm{~d}, J=1.8 \mathrm{~Hz}, \mathrm{H} 12 \mathrm{~b}), 6.11(1 \mathrm{H}$ 
$\mathrm{x} 1 / 2, \mathrm{~d}, J=1.8 \mathrm{~Hz}, \mathrm{H} 12 \mathrm{~b}), 5.45$ (1H, m, H1), 5.11-5.07 (2H, m, $\left.\mathrm{OCH}_{2} \mathrm{OCH}_{3}\right), 4.86(1 \mathrm{H}, \mathrm{m}, \mathrm{H} 8), 4.64$ (1H x1/2, s, H13), 4.60 (1H x1/2, s, H13), 4.18 (1H, m, H2), 4.02 (1H x1/2, dd, J=12.4, $1.8 \mathrm{~Hz}, \mathrm{H} 3 \mathrm{a})$, $4.01(1 \mathrm{H} \mathrm{x} 1 / 2$, dd, $J=12.4,1.8 \mathrm{~Hz}, \mathrm{H} 3 \mathrm{a}), 3.81(1 \mathrm{H}, \mathrm{dd}, J=12.4,3.2 \mathrm{~Hz}, \mathrm{H} 3 \mathrm{~b}), 3.43\left(3 \mathrm{H}, \mathrm{s}, \mathrm{OCH}_{3}\right)$, $3.39\left(3 \mathrm{H} \mathrm{x1} / 2, \mathrm{~s}, \mathrm{OCH}_{3}\right), 3.35\left(3 \mathrm{H} \mathrm{x1} / 2, \mathrm{~s}, \mathrm{OCH}_{3}\right), 2.47(1 \mathrm{H} \mathrm{x} 1 / 2$, ddd, $J=14.2,5.0,1.8 \mathrm{~Hz}, \mathrm{H} 9 \mathrm{a})$, 2.39 (1H x1/2, ddd, $J=14.1,5.0,1.8 \mathrm{~Hz}, \mathrm{H} 9 \mathrm{a}), 2.27$ (1H, m, H9b), 0.91 (9H, s, $t$-Bu of TBS), 0.86 (9H, s, $t$-Bu of TBS), 0.12 (3H, s, CH3 of TBS), 0.09 (3H, s, CH3 of TBS), 0.07 (3H, s, CH3 of TBS), -0.03 (3H, s, $\mathrm{CH}_{3}$ of TBS). HRMS (ESI-TOF) $[\mathrm{M}+\mathrm{Na}]^{+} \mathrm{m} / \mathrm{z}$ : Calcd for $\mathrm{C}_{28} \mathrm{H}_{49} \mathrm{O}_{9} \mathrm{ISi}{ }_{2} \mathrm{Na} 735.1852$; Found 735.1843.

Argon was bubbled through toluene at $26^{\circ} \mathrm{C}$ for $1 \mathrm{~h}$ to remove the residual $\mathrm{O}_{2}$. A solution of the above crude vinyl iodide 23 (4.18 g) and 1,1'-azobis(cyclohexane-1-carbonitrile) (V-40, $143 \mathrm{mg}, 586 \mu \mathrm{mol}$ ) in toluene $(280 \mathrm{~mL})$ was stirred at $125^{\circ} \mathrm{C}$. A solution of $n$-Bu3 $\mathrm{SnH}(4.70 \mathrm{~mL}, 17.4 \mathrm{mmol})$ and V-40 $(148 \mathrm{mg}, 607 \mu \mathrm{mol})$ in toluene $(14.0 \mathrm{~mL})$ was added to the mixture via a syringe pump over $30 \mathrm{~min}$. After the addition was completed, the reaction mixture was stirred at $125{ }^{\circ} \mathrm{C}$ for $1 \mathrm{~h}$. After being cooled to $23{ }^{\circ} \mathrm{C}$, the reaction mixture was directly passed through a column (consecutively packed with silica gel $50 \mathrm{~g}$ and $10 \mathrm{wt} \% \mathrm{KF}$ contained silica gel $100 \mathrm{~g}$, hexane/EtOAc $=20 / 1$ to $1 / 1$ ) and concentrated. The residue was purified by flash column chromatography (a column consecutively packed with silica gel $150 \mathrm{~g}$ and $10 \mathrm{wt} \% \mathrm{KF}$ contained silica gel $50 \mathrm{~g}$, hexane/EtOAc = 15/1) to afford acetals $25-\boldsymbol{\alpha}(1.22 \mathrm{~g}, 2.08 \mathrm{mmol})$ and $\mathbf{2 5 - \beta}(1.27 \mathrm{~g}, 2.17 \mathrm{mmol})$ in $29 \%$ and $30 \%$ yields over 2 steps,

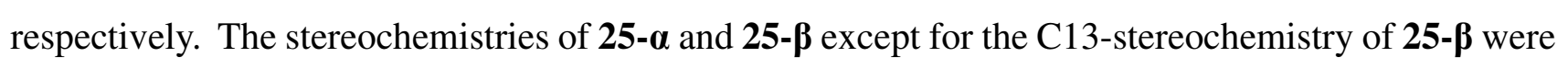
determined by the NOESY experiments, respectively (Page S75, S77). The C13-stereochemistry of 25- $\boldsymbol{\beta}$ was determined after the conversion to 7- $\boldsymbol{\beta}$ (Page S12).

25- $\alpha$ : pale yellow oil. $[\alpha]_{\mathrm{D}}{ }^{19}-25.4$ (c 1.10, $\mathrm{CHCl}_{3}$ ). IR (film): 2953, 2932, 2893, 2859, 1771, 1734 , 1468, 1256, 1120, $1010 \mathrm{~cm}^{-1} .{ }^{1} \mathrm{H}$ NMR (500 MHz, C6 $\left.\mathrm{D}_{6}\right): \delta 6.08(1 \mathrm{H}, \mathrm{br}$ s, H12a), $5.74(1 \mathrm{H}, \mathrm{d}, J=6.9$ Hz, H6), 5.45 (1H, br s, H12b), $5.44(1 \mathrm{H}, \mathrm{d}, J=8.0 \mathrm{~Hz}, \mathrm{H} 1), 5.13(1 \mathrm{H}, \mathrm{s}, \mathrm{H} 13), 4.85$ (1H, ddd, $J=$ 10.9, 5.8, $5.2 \mathrm{~Hz}, \mathrm{H} 8), 4.62\left(1 \mathrm{H}, \mathrm{d}, J=6.3 \mathrm{~Hz}, \mathrm{OCHaHbOCH}_{3}\right), 4.56(1 \mathrm{H}, \mathrm{d}, J=6.3 \mathrm{~Hz}$, $\left.\mathrm{OCHaHbOCH}_{3}\right), 3.81(1 \mathrm{H}, \mathrm{m}, \mathrm{H} 2), 3.71(1 \mathrm{H}, \mathrm{d}, J=12.0 \mathrm{~Hz}, \mathrm{H} 3 \mathrm{a}), 3.58$ (1H, m, H7), 3.53 (1H, dd, $J$ $=12.0,2.3 \mathrm{~Hz}, \mathrm{H} 3 \mathrm{~b}), 3.24\left(3 \mathrm{H}, \mathrm{s}, \mathrm{OCH}_{3}\right), 3.18\left(3 \mathrm{H}, \mathrm{s}, \mathrm{OCH}_{2} \mathrm{OCH}_{3}\right), 2.59(1 \mathrm{H}, \mathrm{dd}, J=14.9,10.9 \mathrm{~Hz}$, H9a), 2.02 (1H, dd, $J=14.9,5.8 \mathrm{~Hz}, \mathrm{H} 9 \mathrm{~b}), 0.94$ (9H, s, $t$-Bu of TBS), 0.93 (9H, s, $t$-Bu of TBS), 0.190 (3H, s, $\mathrm{CH}_{3}$ of TBS), 0.186 (3H, s, $\mathrm{CH}_{3}$ of TBS), 0.04 (3H, s, $\mathrm{CH}_{3}$ of TBS), 0.03 (3H, s, CH3 of TBS). ${ }^{13} \mathrm{C}\left\{{ }^{1} \mathrm{H}\right\}$ NMR $\left(125 \mathrm{MHz}, \mathrm{C}_{6} \mathrm{D}_{6}\right): \delta 199.5,172.4,144.7,114.9,105.2,96.2,84.3,76.1,73.7,67.1,60.4$, 60.1, 56.0, 55.7, 49.5, 31.4, 25.7 (3C), 25.6 (3C), 18.2, 17.8, -4.4, -5.2, -5.4, -5.5. HRMS (ESI-TOF) $[\mathrm{M}+\mathrm{Na}]^{+} \mathrm{m} / \mathrm{z}$ : Calcd for $\mathrm{C}_{28} \mathrm{H}_{50} \mathrm{O}_{9} \mathrm{Si}_{2} \mathrm{Na}$ 609.2886; Found 609.2872.

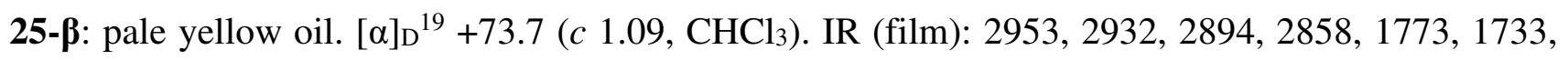
1468, 1255, 1125, $839 \mathrm{~cm}^{-1} .{ }^{1} \mathrm{H}$ NMR (500 MHz, $\left.\mathrm{CDCl}_{3}\right): \delta 5.48(1 \mathrm{H}, \mathrm{s}, \mathrm{H} 12 \mathrm{a}), 5.41(1 \mathrm{H}, \mathrm{s}, \mathrm{H} 12 \mathrm{~b})$, $5.02(1 \mathrm{H}, \mathrm{d}, J=7.5 \mathrm{~Hz}, \mathrm{H} 6), 5.01(1 \mathrm{H}, \mathrm{s}, \mathrm{H} 13), 4.75(1 \mathrm{H}, \mathrm{d}, J=2.3 \mathrm{~Hz}, \mathrm{H} 1), 4.68(1 \mathrm{H}, \mathrm{d}, J=6.9 \mathrm{~Hz}$, 
$\left.\mathrm{OCHaHbOCH}_{3}\right), 4.63(1 \mathrm{H}, \mathrm{ddd}, J=7.5,5.2,2.3 \mathrm{~Hz}, \mathrm{H} 8), 4.54\left(1 \mathrm{H}, \mathrm{d}, J=6.9 \mathrm{~Hz}, \mathrm{OCHaHbOCH}_{3}\right)$, $4.32(1 \mathrm{H}, \mathrm{ddd}, J=6.9,5.8,2.3 \mathrm{~Hz}, \mathrm{H} 2), 3.95(1 \mathrm{H}, \mathrm{dd}, J=10.9,6.9 \mathrm{~Hz}, \mathrm{H} 3 \mathrm{a}), 3.80$ (1H, dd, $J=10.9$, $5.8 \mathrm{~Hz}, \mathrm{H} 3 \mathrm{~b}), 3.68(1 \mathrm{H}, \mathrm{dd}, J=7.5,7.5 \mathrm{~Hz}, \mathrm{H} 7), 3.36\left(3 \mathrm{H}, \mathrm{s}, \mathrm{OCH}_{2} \mathrm{OCH}_{3}\right), 3.35\left(3 \mathrm{H}, \mathrm{s}, \mathrm{OCH}_{3}\right), 2.90$ $(1 \mathrm{H}, \mathrm{dd}, J=15.5,5.2 \mathrm{~Hz}, \mathrm{H} 9 \mathrm{a}), 2.55(1 \mathrm{H}, \mathrm{dd}, J=15.5,2.3 \mathrm{~Hz}, \mathrm{H} 9 \mathrm{~b}), 0.904$ (9H, s, $t$-Bu of TBS), 0.895 (9H, s, $t$-Bu of TBS), 0.15 (3H, s, $\mathrm{CH}_{3}$ of TBS), 0.11 (3H, s, $\mathrm{CH}_{3}$ of TBS), 0.09 (3H, s, $\mathrm{CH}_{3}$ of TBS), $0.08\left(3 \mathrm{H}, \mathrm{s}, \mathrm{CH} 3\right.$ of TBS). ${ }^{13} \mathrm{C}\left\{{ }^{1} \mathrm{H}\right\} \mathrm{NMR}\left(125 \mathrm{MHz}, \mathrm{CDCl}_{3}\right): \delta 201.7,172.9,144.3,115.9$, 104.3, 95.7, 88.0, 74.4, 74.2, 71.6, 62.5, 61.5, 56.1, 55.0, 47.6, 28.4, 25.9 (3C), 25.8 (3C), 18.4, 18.0, $-4.6,-5.2,-5.3,-5.4$. HRMS (ESI-TOF) $[\mathrm{M}+\mathrm{Na}]^{+} \mathrm{m} / \mathrm{z}$ : Calcd for $\mathrm{C}_{28} \mathrm{H}_{50} \mathrm{O}_{9} \mathrm{Si}_{2} \mathrm{Na}$ 609.2886; Found 609.2862 .
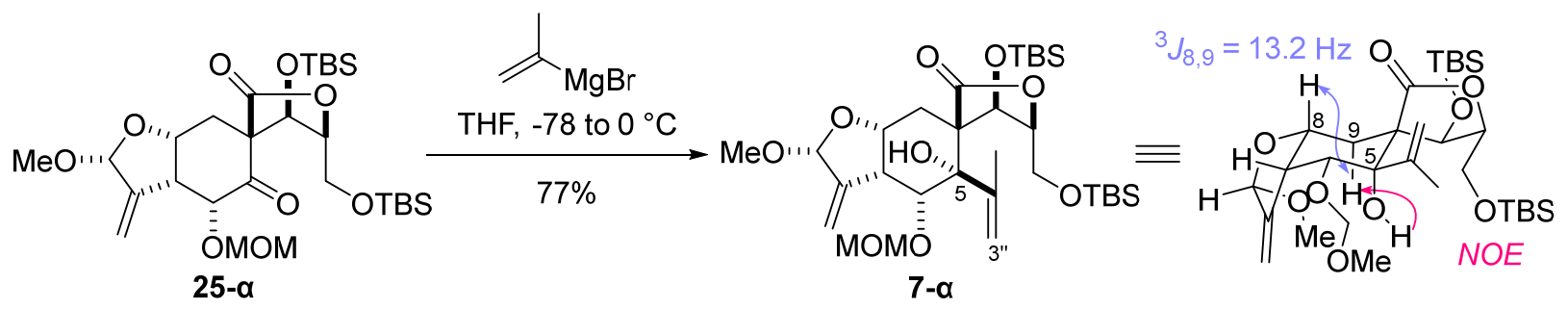

Alcohol 7-a. Isopropenylmagnesium bromide (0.5 M THF solution, $12.0 \mathrm{~mL}, 6.00 \mathrm{mmol}$ ) was added to a solution of acetal $\mathbf{2 5 - \alpha}(1.22 \mathrm{~g}, 2.08 \mathrm{mmol})$ in THF $(21.0 \mathrm{~mL})$ at $-78{ }^{\circ} \mathrm{C}$ over $5 \mathrm{~min}$. After the reaction mixture was stirred at $-78{ }^{\circ} \mathrm{C}$ for $1.5 \mathrm{~h}$ and at $0{ }^{\circ} \mathrm{C}$ for $10 \mathrm{~min}$, saturated aqueous $\mathrm{NH}_{4} \mathrm{Cl}(30$ $\mathrm{mL})$ was added to the mixture. The resultant mixture was extracted with EtOAc $(30 \mathrm{~mL} \times 3)$. The combined organic layers were dried over $\mathrm{Na}_{2} \mathrm{SO}_{4}$, filtered, and concentrated. The residue was purified by flash column chromatography on silica gel (40 g, hexane/EtOAc $=25 / 1$ to $12 / 1)$ to afford alcohol 7- $\boldsymbol{\alpha}(1.01 \mathrm{~g}, 1.61 \mathrm{mmol})$ in $77 \%$ yield. The C5-stereochemistry of 7- $\boldsymbol{\alpha}$ was determined by the NOE experiment in $\mathrm{CO}\left(\mathrm{CD}_{3}\right)_{2}$ (Page S79): white amorphous. [ $\left.\alpha\right]_{\mathrm{D}}{ }^{20}-70.5$ (c 1.07, $\mathrm{CHCl}_{3}$ ). IR (film): 3574 , 2931, 2858, 1766, 1466, 1255, 1134, 1032, 839, $780 \mathrm{~cm}^{-1} .{ }^{1} \mathrm{H} \mathrm{NMR}\left(400 \mathrm{MHz}, \mathrm{CDCl}_{3}\right) \delta 5.87(1 \mathrm{H}, \mathrm{s}$, H12a), 5.41 (1H, s, H3”a), 5.35 (1H, d, J=3.2 Hz, H12b), 5.25 (1H, s, H13), 5.15 (1H, br s, H3”b), $5.00(1 \mathrm{H}, \mathrm{d}, J=7.3 \mathrm{~Hz}, \mathrm{H} 6), 4.77\left(1 \mathrm{H}, \mathrm{d}, J=6.8 \mathrm{~Hz}, \mathrm{OCHaHbOCH}_{3}\right), 4.67-4.58(3 \mathrm{H}, \mathrm{m}, \mathrm{H} 1, \mathrm{H} 8$, $\left.\mathrm{OCHaHbOCH}_{3}\right), 3.99$ (1H, ddd, $\left.J=7.3,3.2,2.3 \mathrm{~Hz}, \mathrm{H} 2\right), 3.92(1 \mathrm{H}, \mathrm{dd}, J=11.9,2.3 \mathrm{~Hz}, \mathrm{H} 3 \mathrm{a}), 3.73$ $(1 \mathrm{H}, \mathrm{dd}, J=11.9,3.2 \mathrm{~Hz}, \mathrm{H} 3 \mathrm{~b}), 3.53(1 \mathrm{H}, \mathrm{m}, \mathrm{H} 7), 3.41\left(3 \mathrm{H}, \mathrm{s}, \mathrm{OCH}_{2} \mathrm{OCH}_{3}\right), 3.38\left(3 \mathrm{H}, \mathrm{s}, \mathrm{OCH}_{3}\right), 2.61$ $(1 \mathrm{H}, \mathrm{s}, \mathrm{OH}), 2.57(1 \mathrm{H}, \mathrm{dd}, J=15.1,11.9 \mathrm{~Hz}, \mathrm{H} 9 \mathrm{a}), 1.77(1 \mathrm{H}, \mathrm{dd}, J=15.1,5.5 \mathrm{~Hz}, \mathrm{H} 9 \mathrm{~b}), 1.75(3 \mathrm{H}, \mathrm{s}$, H15), 0.89 (9H, s, $t$-Bu of TBS), 0.86 (9H, s, $t$-Bu of TBS), 0.10 (3H, s, CH3 of TBS), 0.07 (3H, s, $\mathrm{CH}_{3}$ of TBS), 0.06 (3H, s, CH3 of TBS), 0.04 (3H, s, CH3 of TBS). ${ }^{1} \mathrm{H}$ NMR (500 MHz, $\left.\mathrm{CO}\left(\mathrm{CD}_{3}\right)_{2}\right): \delta 5.83$ (1H, s, H12a), 5.42 (1H, s, H3"'a), 5.26 (1H, br s, H12b), 5.23 (1H, s, H13), 5.15 (1H, s, H3”b), 4.96 $(1 \mathrm{H}, \mathrm{d}, J=6.9 \mathrm{~Hz}, \mathrm{H} 6), 4.81\left(1 \mathrm{H}, \mathrm{d}, J=6.9 \mathrm{~Hz}, \mathrm{OCHaHbOCH}_{3}\right), 4.62-4.61(2 \mathrm{H}, \mathrm{m}, \mathrm{H} 1$, $\left.\mathrm{OCHaHbOCH}_{3}\right), 4.50$ (1H, ddd, $\left.J=13.2,6.3,5.2 \mathrm{~Hz}, \mathrm{H} 8\right), 4.11(1 \mathrm{H}, \mathrm{m}, \mathrm{H} 2), 4.00(1 \mathrm{H}, \mathrm{d}, J=12.0 \mathrm{~Hz}$, H3a), $3.81(1 \mathrm{H}, \mathrm{dd}, J=12.0,3.4 \mathrm{~Hz}, \mathrm{H} 3 \mathrm{~b}), 3.51(1 \mathrm{H}, \mathrm{m}, \mathrm{H} 7), 3.38\left(3 \mathrm{H}, \mathrm{s}, \mathrm{OCH}_{3}\right), 3.36\left(3 \mathrm{H}, \mathrm{s}, \mathrm{OCH}_{3}\right)$, 
$3.07(1 \mathrm{H}, \mathrm{s}, \mathrm{OH}), 2.62(1 \mathrm{H}, \mathrm{dd}, J=14.3,13.2 \mathrm{~Hz}, \mathrm{H} 9 \mathrm{a}), 1.78$ (3H, s, H15), $1.74(1 \mathrm{H}, \mathrm{dd}, J=14.3,5.2$ $\mathrm{Hz}, \mathrm{H} 9 \mathrm{~b}), 0.92$ (9H, s, $t$-Bu of TBS), 0.89 (9H, s, $t$-Bu of TBS), 0.15 (3H, s, CH3 of TBS), 0.11 (3H, s, $\mathrm{CH}_{3}$ of TBS), $0.10\left(6 \mathrm{H}, \mathrm{s}, \mathrm{CH}_{3}\right.$ of TBS x2). ${ }^{13} \mathrm{C}\left\{{ }^{1} \mathrm{H}\right\} \mathrm{NMR}\left(100 \mathrm{MHz}, \mathrm{CDCl}_{3}\right): \delta 176.7,149.4,145.0$, 117.3, 113.5, 105.8, 95.7, 84.4, 80.2, 75.6, 74.1, 70.0, 60.7, 56.2, 55.1, 54.3, 43.0, 27.9, 25.8 (3C), 25.7 (3C), 21.4, 18.4, 18.1, -4.5, -4.6, -5.48, -5.52. HRMS (ESI-TOF) $[\mathrm{M}+\mathrm{Na}]^{+} \mathrm{m} / \mathrm{z}$ : Calcd for $\mathrm{C}_{31} \mathrm{H}_{56} \mathrm{O}_{9} \mathrm{Si}_{2} \mathrm{Na}$ 651.3355; Found 651.3341.

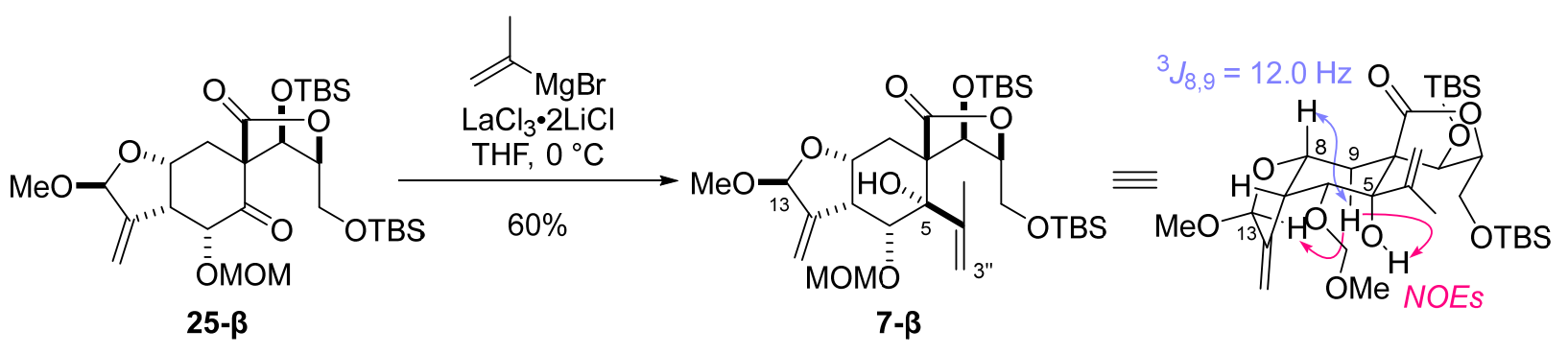

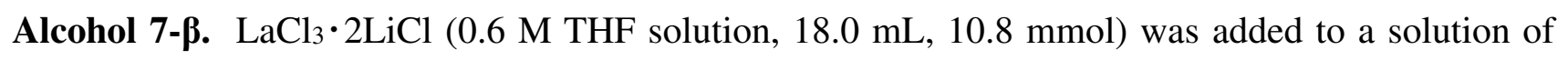
acetal $25-\boldsymbol{\beta}(1.27 \mathrm{~g}, 2.17 \mathrm{mmol})$ in THF $(43.0 \mathrm{~mL})$ at $0{ }^{\circ} \mathrm{C}$. After the mixture was stirred at $0{ }^{\circ} \mathrm{C}$ for 1 $\mathrm{h}$, isopropenylmagnesium bromide $(0.5 \mathrm{M}$ THF solution, $21.0 \mathrm{~mL}, 10.5 \mathrm{mmol})$ was added to the mixture at $0{ }^{\circ} \mathrm{C}$. After the reaction mixture was stirred at $0{ }^{\circ} \mathrm{C}$ for $10 \mathrm{~min}$, saturated aqueous potassium sodium tartrate $(50 \mathrm{~mL})$ was added to the mixture. The resultant mixture was extracted with EtOAc (50 mL x3). The combined organic layers were dried over $\mathrm{Na}_{2} \mathrm{SO}_{4}$, filtered, and concentrated. The residue was purified by flash column chromatography on silica gel $(50 \mathrm{~g}$, hexane/EtOAc $=10 / 1)$ to

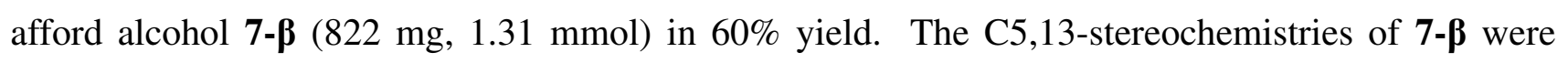
determined by the NOE experiment (Page S81): white amorphous. $[\alpha]_{\mathrm{D}}^{26}+2.22\left(c 1.96, \mathrm{CHCl}_{3}\right)$. IR (film): 3578, 2931, 2858, 1766, 1467, 1254, 1136, 1035, 839, $780 \mathrm{~cm}^{-1} .{ }^{1} \mathrm{H}$ NMR (500 MHz, $\left.\mathrm{C}_{6} \mathrm{D}_{6}\right): \delta$ 5.59 (1H, dd, $J=1.8,1.2 \mathrm{~Hz}, \mathrm{H} 12 \mathrm{a}), 5.52$ (1H, s, H13), 5.45 (1H, d, $J=1.7 \mathrm{~Hz}, \mathrm{H} 3$ " a), 5.26 (1H, d, $J$ $=1.8 \mathrm{~Hz}, \mathrm{H} 12 \mathrm{~b}), 5.18(1 \mathrm{H}, \mathrm{d}, J=7.4 \mathrm{~Hz}, \mathrm{H} 6), 5.10(1 \mathrm{H}, \mathrm{s}, \mathrm{H} 3$ ”'b), $5.01(1 \mathrm{H}, \mathrm{m}, \mathrm{H} 8), 4.72(1 \mathrm{H}, \mathrm{d}, J=$ $7.5 \mathrm{~Hz}, \mathrm{H1}), 4.48\left(1 \mathrm{H}, \mathrm{d}, J=6.9 \mathrm{~Hz}, \mathrm{OCHaHbOCH}_{3}\right), 4.34\left(1 \mathrm{H}, \mathrm{d}, J=6.9 \mathrm{~Hz}, \mathrm{OCHaHbOCH}_{3}\right), 3.74$ $(1 \mathrm{H}, \mathrm{dd}, J=12.0,2.3 \mathrm{~Hz}, \mathrm{H} 3 \mathrm{a}), 3.71(1 \mathrm{H}, \mathrm{ddd}, J=7.5,3.4,2.3 \mathrm{~Hz}, \mathrm{H} 2), 3.64(1 \mathrm{H}, \mathrm{m}, \mathrm{H} 7), 3.61(1 \mathrm{H}$, $\mathrm{dd}, J=12.0,3.4 \mathrm{~Hz}, \mathrm{H} 3 \mathrm{~b}), 3.26\left(3 \mathrm{H}, \mathrm{s}, \mathrm{OCH}_{3}\right), 3.11\left(3 \mathrm{H}, \mathrm{s}, \mathrm{OCH}_{2} \mathrm{OCH}_{3}\right), 2.51(1 \mathrm{H}, \mathrm{s}, \mathrm{OH}), 2.28(1 \mathrm{H}$, dd, $J=14.3,12.0 \mathrm{~Hz}, \mathrm{H} 9 \mathrm{a}), 1.89-1.85$ (4H, m, H9b, H15), 0.95 (9H, s, $t$-Bu of TBS), 0.94 (9H, s, $t$-Bu of TBS), 0.24 (3H, s, CH3 of TBS), $0.12\left(3 \mathrm{H}, \mathrm{s}, \mathrm{CH}\right.$ of TBS), $0.04\left(6 \mathrm{H}, \mathrm{s}, \mathrm{CH}\right.$ of TBS x2). ${ }^{13} \mathrm{C}\left\{{ }^{1} \mathrm{H}\right\}$ NMR (100 MHz, C6 6 6): $\delta$ 175.8, 149.5, 146.3, 117.3, 111.6, 105.1, 95.5, 83.9, 80.5, 75.2, 74.2, 70.5, 60.8, 56.0, 54.2, 52.9, 42.3, 28.5, 26.1 (3C), 26.0 (3C), 22.0, 18.54, 18.46, -4.1, -4.6, -5.3, -5.5. HRMS (ESI-TOF) [M+Na] $]^{+}$m/z: Calcd for $\mathrm{C}_{31} \mathrm{H}_{56} \mathrm{O}_{9} \mathrm{Si}_{2} \mathrm{Na}$ 651.3355; Found 651.3380. 


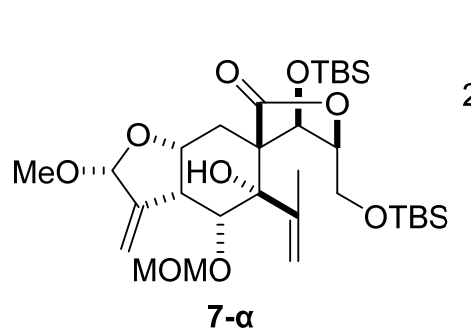

1. $\mathrm{Sc}(\mathrm{OTf})_{3}, \mathrm{Zn}(\mathrm{OTf})_{2}$ $\mathrm{MeCN} / \mathrm{H}_{2} \mathrm{O},-5^{\circ} \mathrm{C}$ (2 cycles)

2. $\mathrm{NaBH}_{4}, \mathrm{CeCl}_{3} \cdot 7 \mathrm{H}_{2} \mathrm{O}$ $\mathrm{MeOH} / \mathrm{CH}_{2} \mathrm{Cl}_{2}, 0$ to $20^{\circ} \mathrm{C}$

$51 \%(2$ steps $)$

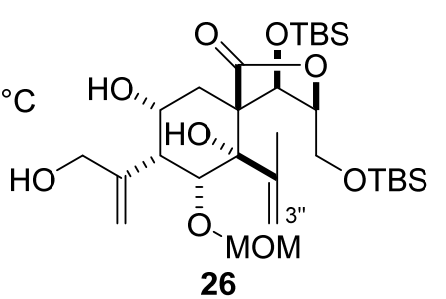

Triol 26 from 7-a. $\mathrm{Zn}(\mathrm{OTf})_{2}(2.93 \mathrm{~g}, 8.05 \mathrm{mmol})$ and $\mathrm{Sc}(\mathrm{OTf})_{3}(79.0 \mathrm{mg}, 161 \mu \mathrm{mol})$ were successively added to a solution of alcohol 7- $\alpha(1.01 \mathrm{~g}, 1.61 \mathrm{mmol})$ in $\mathrm{MeCN}(80.0 \mathrm{~mL})$ and $\mathrm{H}_{2} \mathrm{O}(4.00$ $\mathrm{mL}$ ) at $-5^{\circ} \mathrm{C}$. After the reaction mixture was stirred at $-5^{\circ} \mathrm{C}$ for $3 \mathrm{~h}$, saturated aqueous potassium sodium tartrate $(80 \mathrm{~mL})$ was added to the mixture. The resultant mixture was extracted with $\mathrm{Et}_{2} \mathrm{O}(80$ $\mathrm{mL} \times 3)$. The combined organic layers were washed with brine $(40 \mathrm{~mL})$, dried over $\mathrm{Na}_{2} \mathrm{SO}_{4}$, filtered, and concentrated. The residue was passed through a pad of silica gel ( $40 \mathrm{~g}$, hexane/Et $2 \mathrm{O}=4 / 1$ to $1 / 1$ ) to afford the crude lactol $(425 \mathrm{mg})$ and alcohol 7- $\alpha(516 \mathrm{mg}, 822 \mu \mathrm{mol})$. The procedure was repeated by using the above 7- $\alpha(516 \mathrm{mg}, 822 \mu \mathrm{mol}), \mathrm{Zn}(\mathrm{OTf})_{2}(1.49 \mathrm{~g}, 4.10 \mathrm{mmol})$, and $\mathrm{Sc}(\mathrm{OTf})_{3}(40.2 \mathrm{mg}$, $81.7 \mu \mathrm{mol})$ in $\mathrm{MeCN}(40.0 \mathrm{~mL})$ and $\mathrm{H}_{2} \mathrm{O}(2.00 \mathrm{~mL})$ to afford the crude lactol $(225 \mathrm{mg})$ and alcohol 7 $\boldsymbol{\alpha}(208 \mathrm{mg}, 331 \mu \mathrm{mol})$ in $21 \%$ yield. The combined crude lactol $(650 \mathrm{mg})$ was used in the next reaction without further purification. HRMS (ESI-TOF) $[\mathrm{M}+\mathrm{Na}]^{+} \mathrm{m} / \mathrm{z}$ : Calcd for $\mathrm{C}_{30} \mathrm{H}_{54} \mathrm{O}_{9} \mathrm{Si}_{2} \mathrm{Na}$ 637.3199; Found 637.3226.

$\mathrm{MeOH}(10.0 \mathrm{~mL}), \mathrm{CeCl}_{3} \cdot 7 \mathrm{H}_{2} \mathrm{O}(786 \mathrm{mg}, 2.11 \mathrm{mmol})$, and $\mathrm{NaBH}_{4}(202 \mathrm{mg}, 5.32 \mathrm{mmol})$ were successively added to a solution of the above crude lactol $(650 \mathrm{mg})$ in $\mathrm{CH}_{2} \mathrm{Cl}_{2}(10.0 \mathrm{~mL})$ at $0{ }^{\circ} \mathrm{C}$. After the reaction mixture was stirred at $20^{\circ} \mathrm{C}$ for $2 \mathrm{~h}$, saturated aqueous potassium sodium tartrate $(20 \mathrm{~mL})$ was added to the mixture. After $\mathrm{H}_{2} \mathrm{O}(20 \mathrm{~mL})$ was added to the mixture, the resultant mixture was extracted with $\mathrm{CH}_{2} \mathrm{Cl}_{2}(40 \mathrm{~mL} \times 3)$. The combined organic layers were dried over $\mathrm{Na}_{2} \mathrm{SO}_{4}$, filtered, and concentrated. The residue was purified by flash column chromatography on silica gel (20 g, hexane $/ \mathrm{Et}_{2} \mathrm{O}=1 / 1$ to hexane/EtOAc $\left.=1 / 2\right)$ to afford triol $26(506 \mathrm{mg}, 821 \mu \mathrm{mol})$ in $51 \%$ yield over 2 steps: white amorphous. $[\alpha]_{\mathrm{D}}^{22}-71.9\left(c 1.08, \mathrm{CHCl}_{3}\right)$. IR (film): 3398, 2953, 2930, 2857, 1765, 1467, 1254, 1135, 1032, $838 \mathrm{~cm}^{-1} .{ }^{1} \mathrm{H}$ NMR (400 MHz, $\left.\mathrm{C}_{6} \mathrm{D}_{6}\right): \delta 5.43(1 \mathrm{H}, \mathrm{d}, J=1.8 \mathrm{~Hz}, \mathrm{H} 12 \mathrm{a}), 5.38(1 \mathrm{H}$, d, $J=1.4 \mathrm{~Hz}, \mathrm{H} 3$ ”' a), 5.25 (1H, d, $J=1.8 \mathrm{~Hz}, \mathrm{H} 12 \mathrm{~b}), 5.10$ (1H, dd, $J=1.4,0.9 \mathrm{~Hz}, \mathrm{H} 3$ ”'b), 4.89 (1H, d, $J=7.8 \mathrm{~Hz}, \mathrm{H} 6), 4.79(1 \mathrm{H}, \mathrm{d}, J=7.8 \mathrm{~Hz}, \mathrm{H} 1), 4.56(1 \mathrm{H}, \mathrm{m}, \mathrm{H} 8), 4.42(1 \mathrm{H}, \mathrm{d}, J=6.4 \mathrm{~Hz}$, $\left.\mathrm{OCHaHbOCH}_{3}\right), 4.29\left(1 \mathrm{H}, \mathrm{d}, J=6.4 \mathrm{~Hz}, \mathrm{OCHaHbOCH}_{3}\right), 4.19(1 \mathrm{H}, \mathrm{d}, J=12.8 \mathrm{~Hz}, \mathrm{H} 13 \mathrm{a}), 3.97(1 \mathrm{H}$, d, $J=12.8 \mathrm{~Hz}, \mathrm{H} 13 \mathrm{~b}), 3.76(1 \mathrm{H}, \mathrm{dd}, J=11.9,2.3 \mathrm{~Hz}, \mathrm{H} 3 \mathrm{a}), 3.74(1 \mathrm{H}, \mathrm{ddd}, J=7.8,2.8,2.3 \mathrm{~Hz}, \mathrm{H} 2)$, $3.64(1 \mathrm{H}, \mathrm{dd}, J=11.9,2.8 \mathrm{~Hz}, \mathrm{H} 3 \mathrm{~b}), 3.41(1 \mathrm{H}, \mathrm{dd}, J=7.8,5.9 \mathrm{~Hz}, \mathrm{H} 7), 3.37(1 \mathrm{H}, \mathrm{s}, \mathrm{OH}), 3.06(3 \mathrm{H}$, s, $\left.\mathrm{OCH}_{2} \mathrm{OCH}_{3}\right), 2.85(1 \mathrm{H}, \mathrm{m}, \mathrm{OH}), 2.73(1 \mathrm{H}, \mathrm{dd}, J=15.1,10.5 \mathrm{~Hz}, \mathrm{H} 9 \mathrm{a}), 2.34(1 \mathrm{H}, \mathrm{m}, \mathrm{OH}), 1.93(3 \mathrm{H}$, $\mathrm{d}, J=0.9 \mathrm{~Hz}, \mathrm{H} 15), 1.70(1 \mathrm{H}, \mathrm{dd}, J=15.1,5.5 \mathrm{~Hz}, \mathrm{H} 9 \mathrm{~b}), 0.97$ (9H, s, $t$-Bu of TBS), 0.95 (9H, s, $t$-Bu of TBS), 0.25 (3H, s, CH3 of TBS), 0.12 (3H, s, $\mathrm{CH}_{3}$ of TBS), 0.054 (3H, s, $\mathrm{CH}_{3}$ of TBS), 0.050 (3H, 
s, $\mathrm{CH}_{3}$ of TBS). ${ }^{13} \mathrm{C}\left\{{ }^{1} \mathrm{H}\right\}$ NMR (125 MHz, $\left.\mathrm{C}_{6} \mathrm{D}_{6}\right): \delta 176.3,147.0,145.8,119.0,117.7,96.3,83.6,79.4$, 76.2, 70.3, 68.0, 65.3, 60.7, 56.2, 54.1, 47.4, 30.1, 26.2 (3C), 26.0 (3C), 22.2, 18.52, 18.46, -3.7, -4.5, -5.3, -5.4. HRMS (ESI-TOF) $[\mathrm{M}+\mathrm{Na}]^{+} \mathrm{m} / \mathrm{z}$ : Calcd for $\mathrm{C}_{30} \mathrm{H}_{56} \mathrm{O}_{9} \mathrm{Si}_{2} \mathrm{Na}$ 639.3355; Found 639.3331.

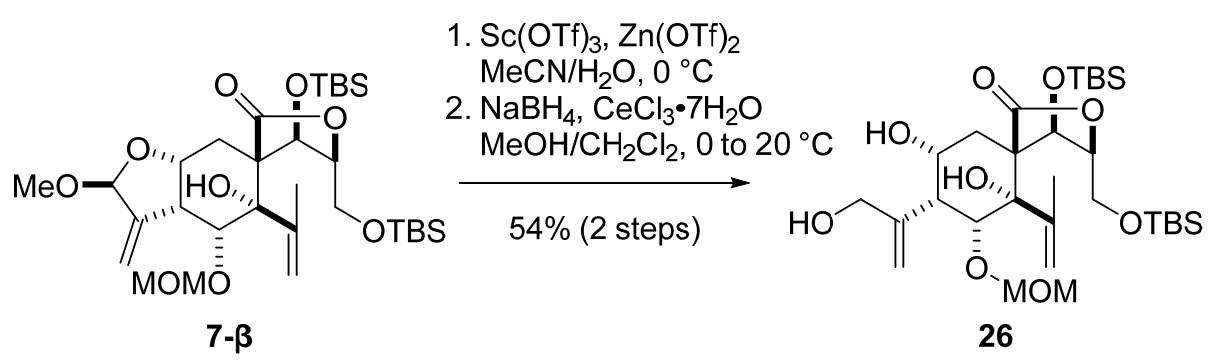

Triol 26 from 7- $\beta$. $\mathrm{Zn}(\mathrm{OTf})_{2}(2.35 \mathrm{~g}, 6.46 \mathrm{mmol})$ and $\mathrm{Sc}(\mathrm{OTf})_{3}(64.4 \mathrm{mg}, 131 \mu \mathrm{mol})$ were successively added to a solution of alcohol 7- $\beta(822 \mathrm{mg}, 1.31 \mathrm{mmol})$ in $\mathrm{MeCN}(65.0 \mathrm{~mL})$ and $\mathrm{H}_{2} \mathrm{O}$ $(6.50 \mathrm{~mL})$ at $0{ }^{\circ} \mathrm{C}$. After the reaction mixture was stirred at $0{ }^{\circ} \mathrm{C}$ for $2.5 \mathrm{~h}$, saturated aqueous potassium sodium tartrate $(70 \mathrm{~mL})$ was added to the mixture. The resultant mixture was extracted with $\mathrm{Et}_{2} \mathrm{O}$ (70 $\mathrm{mL} x 3)$. The combined organic layers were washed with brine $(30 \mathrm{~mL})$, dried over $\mathrm{Na}_{2} \mathrm{SO}_{4}$, filtered, and concentrated. The residue was passed through a pad of silica gel (30 g, hexane/Et ${ }_{2} \mathrm{O}=3 / 1$ to 1/1) to afford the crude lactol $(529 \mathrm{mg})$ and alcohol $7-\boldsymbol{\beta}(103 \mathrm{mg}, 164 \mu \mathrm{mol})$ in $13 \%$ yield. The crude lactol was used in the next reaction without further purification.

$\mathrm{MeOH}(8.50 \mathrm{~mL}), \mathrm{CeCl}_{3} \cdot 7 \mathrm{H}_{2} \mathrm{O}(651 \mathrm{mg}, 1.75 \mathrm{mmol})$, and $\mathrm{NaBH}_{4}(162 \mathrm{mg}, 4.26 \mathrm{mmol})$ were successively added to a solution of the above crude lactol $(529 \mathrm{mg})$ in $\mathrm{CH}_{2} \mathrm{Cl}_{2}(8.50 \mathrm{~mL})$ at $0{ }^{\circ} \mathrm{C}$. After the reaction mixture was stirred at $20^{\circ} \mathrm{C}$ for $2 \mathrm{~h}$, saturated aqueous potassium sodium tartrate $(20 \mathrm{~mL})$ was added to the mixture. After $\mathrm{H}_{2} \mathrm{O}(20 \mathrm{~mL})$ was added to the mixture, the resultant mixture was extracted with $\mathrm{CH}_{2} \mathrm{Cl}_{2}$ (40 mL x3). The combined organic layers were dried over $\mathrm{Na}_{2} \mathrm{SO}_{4}$, filtered, and concentrated. The residue was purified by flash column chromatography on silica gel (15 g, hexane $/ \mathrm{Et}_{2} \mathrm{O}=1 / 1$ to hexane/EtOAc $\left.=1 / 2\right)$ to afford triol $26(433 \mathrm{mg}, 703 \mu \mathrm{mol})$ in $54 \%$ yield over 2 steps. 


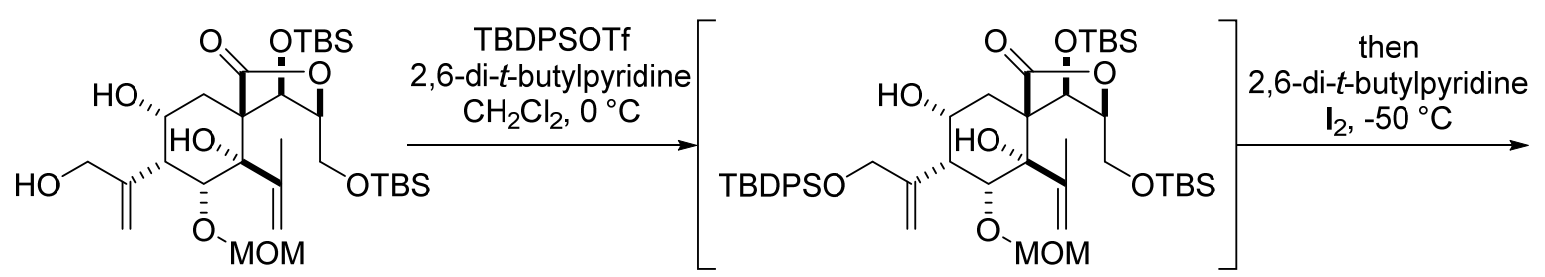

26

27

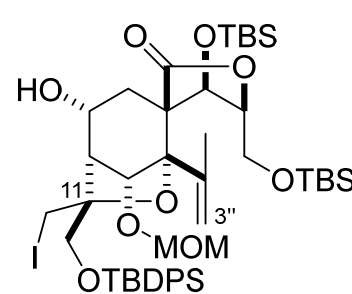

29

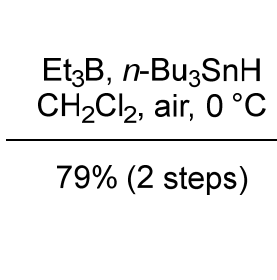

$\mathrm{Et}_{3} \mathrm{~B}, n-\mathrm{Bu}_{3} \mathrm{SnH}$ ${ }_{2} \mathrm{Cl}_{2}$, air, $0^{\circ} \mathrm{C}$

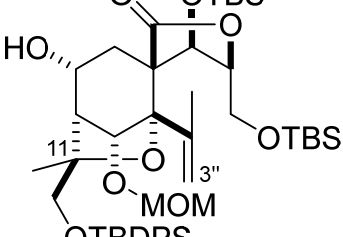

OTBDPS

30

C-ring 30. 2,6-Di-tert-butylpyridine $(830 \mu \mathrm{L}, 3.69 \mathrm{mmol})$ and tert-butyldiphenylsilyl trifluoromethanesulfonate (TBDPSOTf, $540 \mu \mathrm{L}, 1.75 \mathrm{mmol}$ ) were successively added to a solution of triol 26 (909 mg, $1.48 \mathrm{mmol})$ in $\mathrm{CH}_{2} \mathrm{Cl}_{2}(30.0 \mathrm{~mL})$ at $0{ }^{\circ} \mathrm{C}$. After the reaction mixture was stirred at $0{ }^{\circ} \mathrm{C}$ for $10 \mathrm{~min}, 2,6$-di-tert-butylpyridine $(1.20 \mathrm{~mL}, 5.33 \mathrm{mmol})$ and $\mathrm{I}_{2}(1.12 \mathrm{~g}, 4.41 \mathrm{mmol})$ were successively added to the mixture at $-50{ }^{\circ} \mathrm{C}$. After the reaction mixture was stirred at $-50{ }^{\circ} \mathrm{C}$ for $17 \mathrm{~h}$, a 1:1 mixture of saturated aqueous $\mathrm{NaHCO}_{3}$ and saturated aqueous $\mathrm{Na}_{2} \mathrm{~S}_{2} \mathrm{O}_{3}(30 \mathrm{~mL})$ was added to the mixture. The resultant mixture was extracted with $\mathrm{CH}_{2} \mathrm{Cl}_{2}(30 \mathrm{~mL}$ x3). The combined organic layers were dried over $\mathrm{Na}_{2} \mathrm{SO}_{4}$, filtered, and concentrated to afford the crude iodide $\mathbf{2 9}$, which was used in the next reaction without further purification. To collect the analytical data, $\mathbf{2 9}$ was partially purified by PTLC $\left(10 \mathrm{~cm} \mathrm{x} 10 \mathrm{~cm}, \mathrm{CH}_{2} \mathrm{Cl}_{2} / \mathrm{EtOAc}=100 / 1\right.$, developed twice $)$. The C11-stereochemistry of 29 was determined after the conversion to 30 (Page S16): white amorphous. $[\alpha]_{\mathrm{D}}{ }^{20}-43.6\left(c 0.133, \mathrm{CHCl}_{3}\right)$. IR (film): 3481, 2929, 2857, 1764, 1468, 1255, 1134, 1110, 1071, $1035 \mathrm{~cm}^{-1} .{ }^{1} \mathrm{H}$ NMR (400 MHz, $\left.\mathrm{C}_{6} \mathrm{D}_{6}\right): \delta$ 8.00-7.98 (2H, m, aromatic), 7.92-7.90 (2H, m, aromatic), 7.28-7.23 (6H, m, aromatic), 5.76 (1H, d, $J=2.3 \mathrm{~Hz}, \mathrm{H} 3$ ”' a), 5.57 (1H, s, H6), 5.06 (1H, s, H3”b), 4.85 (1H, d, J = 10.5 Hz, H13a), 4.73 $(1 \mathrm{H}, \mathrm{d}, J=6.9 \mathrm{~Hz}, \mathrm{H} 1), 4.46(1 \mathrm{H}, \mathrm{d}, J=8.7 \mathrm{~Hz}, \mathrm{H} 12 \mathrm{a}), 4.29\left(1 \mathrm{H}, \mathrm{d}, J=6.8 \mathrm{~Hz}, \mathrm{OCHaHbOCH}_{3}\right), 4.27$ $(1 \mathrm{H}, \mathrm{d}, J=10.5 \mathrm{~Hz}, \mathrm{H} 13 \mathrm{a}), 4.23-4.21$ (2H, m, H12b, OCHaHbOCH$), 4.09$ (1H, m, H8), $3.72(1 \mathrm{H}, \mathrm{dd}$, $J=11.9,1.8 \mathrm{~Hz}, \mathrm{H} 3 \mathrm{~b}), 3.62-3.60(2 \mathrm{H}, \mathrm{m}, \mathrm{H} 2, \mathrm{H} 3 \mathrm{a}), 2.93\left(3 \mathrm{H}, \mathrm{s}, \mathrm{OCH}_{2} \mathrm{OCH}_{3}\right), 2.70(1 \mathrm{H}, \mathrm{dd}, J=14.1$, $11.4 \mathrm{~Hz}, \mathrm{H} 9 \mathrm{a}), 2.53$ (1H, d, $J=2.7 \mathrm{~Hz}, \mathrm{H} 7), 1.75$ (3H, s, H15), 1.55 (1H, dd, J = 14.1, $6.9 \mathrm{~Hz}, \mathrm{H} 9 \mathrm{~b})$, $1.33(9 \mathrm{H}, \mathrm{s}, t$-Bu of TBDPS), $1.25(1 \mathrm{H}, \mathrm{d}, J=3.2 \mathrm{~Hz}, \mathrm{OH}), 0.93(9 \mathrm{H}, \mathrm{s}, t$-Bu of TBS), 0.91 (9H, s, $t$ Bu of TBS), 0.39 (3H, s, CH3 of TBS), $0.10\left(3 \mathrm{H}, \mathrm{s}, \mathrm{CH}_{3}\right.$ of TBS), 0.04 (3H, s, $\mathrm{CH}_{3}$ of TBS), 0.03 (3H, s, $\mathrm{CH}_{3}$ of TBS). ${ }^{13} \mathrm{C}\left\{{ }^{1} \mathrm{H}\right\}$ NMR (100 MHz, $\left.\mathrm{C}_{6} \mathrm{D}_{6}\right): \delta$ 176.8, 141.7, 136.7 (2C), 136.6 (2C), 134.05, 133.98, 130.0, 129.8, 128 (4C, deduced from HMQC), 117.0, 97.0, 94.6, 88.1, 82.2, 80.3, 69.7, 69.5, 67.2, 60.0, 56.0, 52.8, 51.1, 30.3, 27.4 (3C), 26.1 (3C), 26.0 (3C), 21.3, 19.6, 18.5, 18.3, 7.5, -2.6, 4.4, -5.3, -5.4. HRMS (ESI-TOF) $[\mathrm{M}+\mathrm{Na}]^{+} \mathrm{m} / \mathrm{z}$ : Calcd for $\mathrm{C}_{46} \mathrm{H}_{73} \mathrm{IO}_{9} \mathrm{Si}_{3} \mathrm{Na}$ 1003.3499; Found 1003.3488. 
$n$-Bu $\mathrm{Bu}_{3} \mathrm{SnH}(1.20 \mathrm{~mL}, 4.45 \mathrm{mmol})$ and $\mathrm{Et}_{3} \mathrm{~B}$ (0.99 M hexane solution, $\left.3.00 \mathrm{~mL}, 2.97 \mathrm{mmol}\right)$ were successively added to a solution of the above crude 29 in $\mathrm{CH}_{2} \mathrm{Cl}_{2}(15.0 \mathrm{~mL})$ at $0{ }^{\circ} \mathrm{C}$ under air. After being stirred at $0{ }^{\circ} \mathrm{C}$ for 10 min under air, the reaction mixture was directly passed through a column (consecutively packed with silica gel $15 \mathrm{~g}$ and $10 \mathrm{wt} \% \mathrm{KF}$ contained silica gel $30 \mathrm{~g}$, hexane/EtOAc = $20 / 1$ to $1 / 2$ ) and concentrated. The residue was purified by flash column chromatography (a column consecutively packed with silica gel $30 \mathrm{~g}$ and $10 \mathrm{wt} \% \mathrm{KF}$ contained silica gel $10 \mathrm{~g}$, hexane/EtOAc $=$ 10/1 to 5/1) to afford C-ring $30(1.00 \mathrm{~g}, 1.17 \mathrm{mmol})$ in $79 \%$ yield over 2 steps. The C11stereochemistry of $\mathbf{3 0}$ was determined by the NOE experiment (Page S85): white amorphous. $[\alpha]_{\mathrm{D}}{ }^{26}-$ 76.1 (c 1.00, $\mathrm{CHCl}_{3}$ ). IR (film): 3486, 2932, 2859, 1763, 1467, 1256, 1111, 1076, 1034, $837 \mathrm{~cm}^{-1} .{ }^{1} \mathrm{H}$ NMR (400 MHz, $\mathrm{C}_{6} \mathrm{D}_{6}, 1: 0.08$ conformational mixture) peaks of the major isomer: $\delta 7.84-7.80(4 \mathrm{H}$, m, aromatic), 7.22-7.20 (6H, m, aromatic), 5.77 (1H, d, J=2.8 Hz, H3"'a), 5.66 (1H, s, H6), 5.11 (1H, $\mathrm{dd}, J=2.8,1.4 \mathrm{~Hz}, \mathrm{H} 3$ ”' $), 4.75(1 \mathrm{H}, \mathrm{d}, J=7.8 \mathrm{~Hz}, \mathrm{H} 1), 4.57\left(1 \mathrm{H}, \mathrm{d}, J=6.8 \mathrm{~Hz}, \mathrm{OCHaHbOCH}_{3}\right)$, $4.44\left(1 \mathrm{H}, \mathrm{d}, J=6.8 \mathrm{~Hz}, \mathrm{OCHaHbOCH}_{3}\right), 4.19-4.12$ (2H, m, H8, H13a), 4.07 (1H, d, $\left.J=9.6 \mathrm{~Hz}, \mathrm{H} 13 \mathrm{~b}\right)$, $3.77(1 \mathrm{H}, \mathrm{dd}, J=11.9,1.8 \mathrm{~Hz}, \mathrm{H} 3 \mathrm{a}), 3.67(1 \mathrm{H}, \mathrm{ddd}, J=7.8,2.3,1.8 \mathrm{~Hz}, \mathrm{H} 2), 3.64(1 \mathrm{H}, \mathrm{dd}, J=11.9$, $2.3 \mathrm{~Hz}, \mathrm{H} 3 \mathrm{~b}), 3.12\left(3 \mathrm{H}, \mathrm{s}, \mathrm{OCH}_{2} \mathrm{OCH}_{3}\right), 2.85(1 \mathrm{H}, \mathrm{dd}, J=14.2,11.9 \mathrm{~Hz}, \mathrm{H} 9 \mathrm{a}), 2.62(1 \mathrm{H}, \mathrm{d}, J=2.7$ Hz, H7), 1.95 (3H, s, H12), 1.85 (3H, s, H15), 1.50 (1H, dd, $J=14.2,6.8 \mathrm{~Hz}, \mathrm{H} 9 \mathrm{~b}), 1.24$ (9H, s, $t$-Bu of TBDPS), 0.95 (9H, s, $t$-Bu of TBS), 0.94 (9H, s, $t$-Bu of TBS), 0.34 (3H, s, CH3 of TBS), 0.10 (3H, s, $\mathrm{CH}_{3}$ of TBS), 0.054 (3H, s, $\mathrm{CH}_{3}$ of TBS), 0.046 (3H, s, CH3 of TBS). ${ }^{13} \mathrm{C}\left\{{ }^{1} \mathrm{H}\right\} \mathrm{NMR}(100 \mathrm{MHz}$, $\mathrm{C}_{6} \mathrm{D}_{6}$ ) peaks of the major isomer: $\delta 177.0,142.2,136.3$ (2C), 136.1 (2C), 134.4, 134.1, 129.8 (2C), 128 (4C, deduced from HMQC), 116.6, 96.4, 93.2, 86.2, 82.1, 80.0, 70.7, 69.8, 69.5, 60.0, 56.0, 53.0, 50.7, 31.1, 27.2 (3C), 26.1 (3C), 26.0 (3C), 21.6, 21.4, 19.6, 18.6, 18.3, -2.8, -4.4, -5.3, -5.4. HRMS (ESI-TOF) $[\mathrm{M}+\mathrm{Na}]^{+} \mathrm{m} / \mathrm{z}$ : Calcd for $\mathrm{C}_{46} \mathrm{H}_{74} \mathrm{O} 9 \mathrm{Si}_{3} \mathrm{Na}$ 877.4533; Found 877.4541.

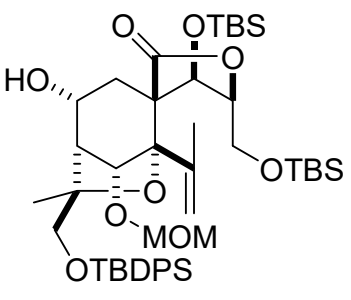

30

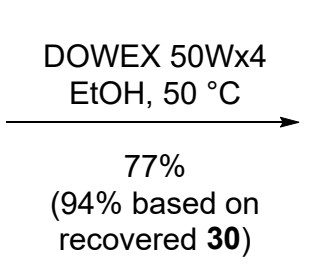

$(94 \%$ based on
recovered 30 )

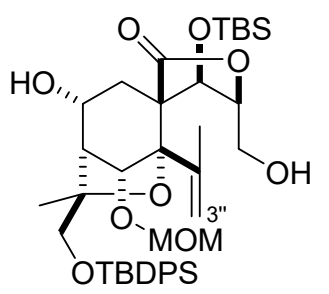

31

Diol 31. DOWEX 50Wx4 (3.01 g) was added to a solution of C-ring 30 (1.00 g, $1.17 \mathrm{mmol})$ in EtOH $(12.0 \mathrm{~mL})$ at $23^{\circ} \mathrm{C}$. After the reaction mixture was stirred at $50{ }^{\circ} \mathrm{C}$ for $24 \mathrm{~h}, \mathrm{CHCl}_{3}(60 \mathrm{~mL})$ was added to the mixture at $23{ }^{\circ} \mathrm{C}$. The resultant mixture was directly passed through a pad of silica gel (20 g, $\left.\mathrm{CHCl}_{3} / \mathrm{MeOH}=5 / 1\right)$ and concentrated. The residue was purified by flash column chromatography on silica gel (30 g, hexane/EtOAc $=20 / 1$ to $4 / 1$ to $2 / 1)$ to afford diol $31(668 \mathrm{mg}, 903 \mu \mathrm{mol})$ and C-ring $30(175 \mathrm{mg}, 205 \mu \mathrm{mol})$ in $77 \%$ and $18 \%$ yields, respectively: white amorphous. $[\alpha]_{\mathrm{D}}{ }^{20}-80.5(c 1.15$, $\mathrm{CHCl}_{3}$ ). IR (film): 3447, 2930, 2857, 1747, 1472, 1259, 1153, 1082, 1036, $837 \mathrm{~cm}^{-1} .{ }^{1} \mathrm{H}$ NMR (400 
$\mathrm{MHz}, \mathrm{C}_{6} \mathrm{D}_{6}, 1: 0.09$ conformational mixture) peaks of the major isomer: $\delta 7.83-7.79(4 \mathrm{H}$, m, aromatic), 7.22-7.18 (6H, m, aromatic), 5.73 (1H, d, $J=2.7 \mathrm{~Hz}, \mathrm{H} 3$ ”a), 5.62 (1H, s, H6), 5.04 (1H, m, H3”b), $4.62(1 \mathrm{H}, \mathrm{d}, J=7.8 \mathrm{~Hz}, \mathrm{H} 1), 4.57\left(1 \mathrm{H}, \mathrm{d}, J=6.4 \mathrm{~Hz}, \mathrm{OCHaHbOCH}_{3}\right), 4.44(1 \mathrm{H}, \mathrm{d}, J=6.4 \mathrm{~Hz}$, $\left.\mathrm{OCHaHbOCH}_{3}\right), 4.19-4.10$ (2H, m, H8, H13a), 4.03 (1H, d, $\left.J=9.6 \mathrm{~Hz}, \mathrm{H} 13 \mathrm{~b}\right), 3.63$ (1H, ddd, $J=7.8$, 3.2, $3.2 \mathrm{~Hz}, \mathrm{H} 2), 3.45(1 \mathrm{H}, \mathrm{m}, \mathrm{H} 3 \mathrm{a}), 3.36(1 \mathrm{H}, \mathrm{m}, \mathrm{H} 3 \mathrm{~b}), 3.13\left(3 \mathrm{H}, \mathrm{s}, \mathrm{OCH}_{2} \mathrm{OCH}_{3}\right), 2.81(1 \mathrm{H}, \mathrm{dd}, J=$ 14.6, 11.9 Hz, H9a), 2.63 (1H, d, J = 2.7 Hz, H7), 1.93 (3H, s, H12), 1.75 (3H, s, H15), 1.47 (1H, dd, $J=14.6,6.4 \mathrm{~Hz}, \mathrm{H} 9 \mathrm{~b}), 1.23$ (9H, s, $t$-Bu of TBDPS), 0.91 (9H, s, $t$-Bu of TBS), 0.29 (3H, s, CH3 of TBS), 0.03 (3H, s, $\mathrm{CH}_{3}$ of TBS). ${ }^{13} \mathrm{C}\left\{{ }^{1} \mathrm{H}\right\}$ NMR (125 MHz, $\left.\mathrm{C}_{6} \mathrm{D}_{6}\right)$ peaks of the major isomer: $\delta 177.7$, 141.9, 136.3 (2C), 136.1 (2C), 134.4, 134.1, 129.8 (2C), 128 (4C, deduced from HMQC), 117.0, 96.3, 93.2, 86.3, 82.8, 79.9, 70.7, 70.2, 69.5, 59.6, 56.0, 53.2, 50.7, 31.3, 27.2 (3C), 26.1 (3C), 21.4, 20.9, 19.6, 18.3, -2.9, -4.5. HRMS (ESI-TOF) $[\mathrm{M}+\mathrm{Na}]^{+} \mathrm{m} / \mathrm{z}$ : Calcd for $\mathrm{C}_{40} \mathrm{H}_{60} \mathrm{O}_{9} \mathrm{Si}_{2} \mathrm{Na} 763.3668$; Found 763.3646.
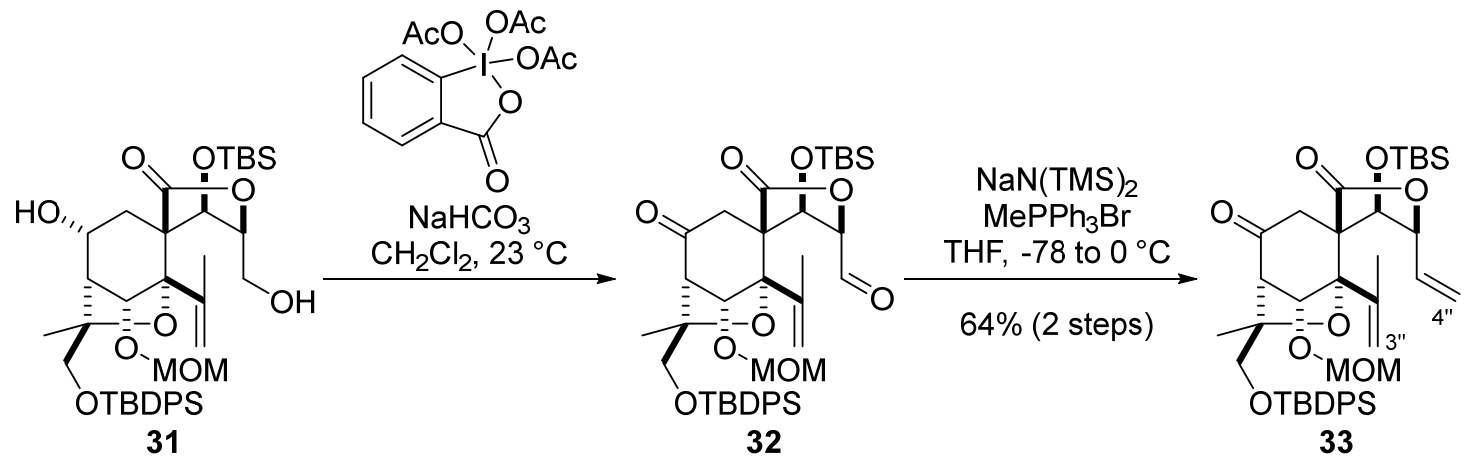

Diene 33. Dess-Martin periodinane (293 mg, $691 \mu \mathrm{mol}$ ) was added to a suspension of diol 31 (202 $\mathrm{mg}, 273 \mu \mathrm{mol})$ and $\mathrm{NaHCO}_{3}(117 \mathrm{mg}, 1.39 \mathrm{mmol})$ in $\mathrm{CH}_{2} \mathrm{Cl}_{2}(6.80 \mathrm{~mL})$ at $0{ }^{\circ} \mathrm{C}$. After the reaction mixture was stirred at $23{ }^{\circ} \mathrm{C}$ for $1 \mathrm{~h}$, saturated aqueous $\mathrm{NaHCO}_{3}(5 \mathrm{~mL})$ and saturated aqueous $\mathrm{Na}_{2} \mathrm{~S}_{2} \mathrm{O}_{3}$ $(5 \mathrm{~mL})$ were successively added to the mixture at $0{ }^{\circ} \mathrm{C}$. The resultant mixture was extracted with $\mathrm{CH}_{2} \mathrm{Cl}_{2}$ (10 mL x4). The combined organic layers were dried over $\mathrm{Na}_{2} \mathrm{SO}_{4}$, filtered, and concentrated to afford the crude aldehyde $32(271 \mathrm{mg})$, which was used in the next reaction without further purification. HRMS (ESI-TOF) $[\mathrm{M}+\mathrm{MeOH}+\mathrm{Na}]^{+} \mathrm{m} / \mathrm{z}$ : Calcd for $\mathrm{C}_{41} \mathrm{H}_{60} \mathrm{O}_{10} \mathrm{Si}_{2} \mathrm{Na}$ 791.3617; Found 791.3593.

Sodium bis(trimethylsilyl)amide (NaN(TMS)2, THF solution, $1.40 \mathrm{~mL}, 1.40 \mathrm{mmol}$ ) was added dropwise to a suspension of $\mathrm{MePPh}_{3} \mathrm{Br}$ (dried over $\mathrm{P}_{2} \mathrm{O}_{5}, 538 \mathrm{mg}, 1.51 \mathrm{mmol}$ ) in THF (6.50 mL) at $0{ }^{\circ} \mathrm{C}$. After the mixture was stirred at $23{ }^{\circ} \mathrm{C}$ for $30 \mathrm{~min}$, a solution of the above crude aldehyde 32 $(271 \mathrm{mg})$ in THF $(7.00 \mathrm{~mL})$ was added dropwise to the mixture at $-78{ }^{\circ} \mathrm{C}$ via cannula. After the reaction mixture was stirred at $-78^{\circ} \mathrm{C}$ for $30 \mathrm{~min}$ and at $0{ }^{\circ} \mathrm{C}$ for $30 \mathrm{~min}$, saturated aqueous $\mathrm{NH}_{4} \mathrm{Cl}(15$ $\mathrm{mL})$ was added to the mixture. The resultant mixture was extracted with EtOAc $(15 \mathrm{~mL} \times 3)$. The combined organic layers were dried over $\mathrm{Na}_{2} \mathrm{SO}_{4}$, filtered, and concentrated. The residue was purified 
by automated flash column chromatography using the prepacked silica gel $(16 \mathrm{~g} \mathrm{x} 2$, hexane/EtOAc $=$ 96/4 to 75/25) to afford diene 33 (129 mg, $176 \mu \mathrm{mol})$ in 64\% yield over 2 steps: white amorphous. $[\alpha]_{\mathrm{D}}^{23}-32.8$ (c 0.500, $\left.\mathrm{CHCl}_{3}\right)$. IR (film): 2954, 2925, 2854, 1769, 1729, 1470, 1428, 1257, 1153, 1110 $\mathrm{cm}^{-1} .{ }^{1} \mathrm{H}$ NMR (400 MHz, C6 6 ): $\delta$ 7.76-7.71 (4H, m, aromatic), 7.20-7.16 (6H, m, aromatic), 5.96 (1H, s, H6), 5.64 (1H, m, H3”'a), 5.60 (1H, ddd, $J=17.4,9.6,7.3 \mathrm{~Hz}, \mathrm{H} 3), 5.18$ (1H, dd, $J=17.4,0.9$ Hz, H4”a), 5.03-5.00 (2H, m, H3”b, H4”b), 4.39 (1H, d, $J=6.4$ Hz, H1), 4.31-4.27 (2H, m, $\left.\mathrm{OCH}_{2} \mathrm{OCH}_{3}\right), 4.07-4.00(2 \mathrm{H}, \mathrm{m}, \mathrm{H} 13), 3.95(1 \mathrm{H}, \mathrm{dd}, J=7.3,6.4 \mathrm{~Hz}, \mathrm{H} 2), 3.44(1 \mathrm{H}, \mathrm{s}, \mathrm{H} 7), 3.40(1 \mathrm{H}$, d, $J=18.3 \mathrm{~Hz}, \mathrm{H} 9 \mathrm{a}), 2.96\left(3 \mathrm{H}, \mathrm{s}, \mathrm{OCH}_{2} \mathrm{OCH}_{3}\right), 2.38(1 \mathrm{H}, \mathrm{d}, J=18.3 \mathrm{~Hz}, \mathrm{H} 9 \mathrm{~b}), 1.73$ (3H, s, H15), 1.54 (3H, s, H12), 1.17 (9H, s, $t$-Bu of TBDPS), 0.86 (9H, s, $t$-Bu of TBS), 0.17 (3H, s, $\mathrm{CH}_{3}$ of TBS), -0.06 (3H, s, $\mathrm{CH}_{3}$ of TBS). ${ }^{13} \mathrm{C}\left\{{ }^{1} \mathrm{H}\right\}$ NMR $\left(125 \mathrm{MHz}, \mathrm{C}_{6} \mathrm{D}_{6}\right): \delta 203.5,176.5,141.7,136.2(2 \mathrm{C}), 136.0$ (2C), 133.9, 133.7, 133.6, 130.0 (2C), 128.5 (4C), 120.9, 117.9, 96.9, 94.5, 85.9, 84.2, 79.0, 75.2, 69.7, 62.7, 56.1, 53.4, 40.1, 27.1 (3C), 26.0 (3C), 21.3, 20.9, 19.5, 18.1, -3.40, -3.43. HRMS (ESI-TOF) $[\mathrm{M}+\mathrm{Na}]^{+} \mathrm{m} / \mathrm{z}$ : Calcd for $\mathrm{C}_{41} \mathrm{H}_{58} \mathrm{O}_{8} \mathrm{Si}_{2} \mathrm{Na} 757.3562$; Found 757.3576 .

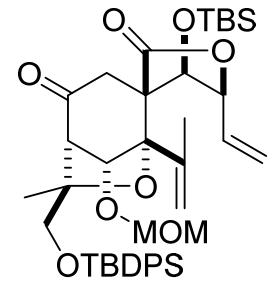

33

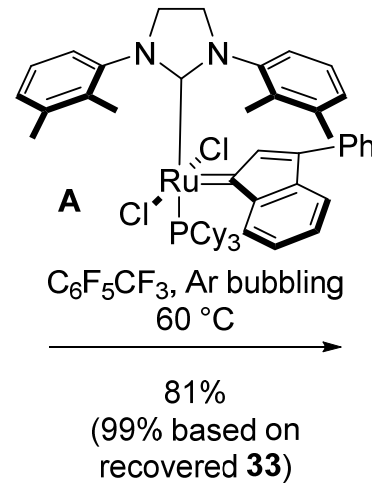

recovered 33)

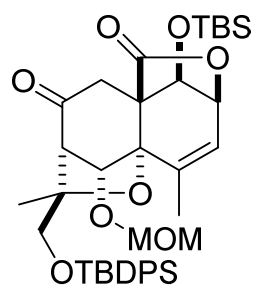

6

A-ring 6. Argon was bubbled through a solution of diene $33(169 \mathrm{mg}, 230 \mu \mathrm{mol})$ in $\mathrm{C}_{6} \mathrm{~F}_{5} \mathrm{CF}_{3}(5.80$ $\mathrm{mL})$ at $25{ }^{\circ} \mathrm{C}$ for $30 \mathrm{~min}$ to remove the residual $\mathrm{O}_{2}$. Ru-catalyst $\mathbf{A}(5.3 \mathrm{mg}, 5.7 \mu \mathrm{mol})$ was added to the mixture. Argon bubbling was continued during the reaction. After the reaction mixture was stirred at $60{ }^{\circ} \mathrm{C}$ for $2 \mathrm{~h}$, Ru-catalyst $\mathbf{A}(5.3 \mathrm{mg}, 5.7 \mu \mathrm{mol})$ was added to the mixture at $25^{\circ} \mathrm{C}$. After the reaction mixture was stirred at $60{ }^{\circ} \mathrm{C}$ for $2 \mathrm{~h}$, Ru-catalyst $\mathbf{A}(5.3 \mathrm{mg}, 5.7 \mu \mathrm{mol})$ was added to the mixture at $25{ }^{\circ} \mathrm{C}$. After the reaction mixture was stirred at $60{ }^{\circ} \mathrm{C}$ for $2 \mathrm{~h}$, Ru-catalyst $\mathbf{A}(5.3 \mathrm{mg}, 5.7 \mu \mathrm{mol})$ was added to the mixture at $25^{\circ} \mathrm{C}$. The reaction mixture was stirred at $60{ }^{\circ} \mathrm{C}$ for $19 \mathrm{~h}$. After being cooled to $25^{\circ} \mathrm{C}$, the reaction mixture was directly passed through a column (consecutively packed with silica gel $6 \mathrm{~g}$ and $10 \mathrm{wt} \% \mathrm{KF}$ contained silica gel $2 \mathrm{~g}$, hexane/EtOAc $=5 / 1)$ and concentrated. The residue was purified by automated flash column chromatography using the prepacked silica gel (40 g x2, hexane/EtOAc $=96 / 4$ to $75 / 25)$ to afford pure A-ring $6(111 \mathrm{mg}, 157 \mu \mathrm{mol})$, along with a mixture of A-ring $\mathbf{6}$ and diene 33 (33.4 mg), and pure diene 33 (19.6 mg, $26.7 \mu \mathrm{mol})$. The mixture of $\mathbf{6}$ and 33 (33.4 mg) was purified by automated flash column chromatography using the prepacked silica gel (16 
$\mathrm{g} \mathrm{x} 2$, hexane/EtOAc $=96 / 4$ to 75/25) to afford A-ring $6(20.5 \mathrm{mg}, 29.0 \mu \mathrm{mol})$ and diene $33(10.5 \mathrm{mg}$, $14.3 \mu \mathrm{mol})$. A-ring 6 (131 mg, $186 \mu \mathrm{mol})$ and diene 33 (30.1 mg, $41.0 \mu \mathrm{mol})$ were obtained in 81\% and $18 \%$ yields, respectively: white amorphous. $[\alpha]_{\mathrm{D}}{ }^{25}+44.4\left(c 1.20, \mathrm{CHCl}_{3}\right)$. IR (film): 2932, 2890, 2858, 1774, 1727, 1469, 1257, 1106, 1011, $989 \mathrm{~cm}^{-1} .{ }^{1} \mathrm{H}$ NMR (500 MHz, C6 6$): \delta 7.73-7.70$ (4H, m, aromatic), 7.22-7.17 (6H, m, aromatic), $5.62(1 \mathrm{H}, \mathrm{dd}, J=6.3,1.2 \mathrm{~Hz}, \mathrm{H} 3), 5.06(1 \mathrm{H}, \mathrm{s}, \mathrm{H} 6), 4.45(1 \mathrm{H}$, s, H1), $4.28(1 \mathrm{H}, \mathrm{d}, J=9.8 \mathrm{~Hz}, \mathrm{H} 13 \mathrm{a}), 4.16(1 \mathrm{H}, \mathrm{d}, J=6.3 \mathrm{~Hz}, \mathrm{H} 2), 4.09(1 \mathrm{H}, \mathrm{d}, J=6.3 \mathrm{~Hz}$, $\left.\mathrm{OCHaHbOCH}_{3}\right), 4.07\left(1 \mathrm{H}, \mathrm{d}, J=6.3 \mathrm{~Hz}, \mathrm{OCHaHbOCH}_{3}\right), 3.78(1 \mathrm{H}, \mathrm{d}, J=9.8 \mathrm{~Hz}, \mathrm{H} 13 \mathrm{~b}), 3.50(1 \mathrm{H}$, s, H7), $3.18(1 \mathrm{H}, \mathrm{d}, J=18.3 \mathrm{~Hz}, \mathrm{H} 9 \mathrm{a}), 2.92\left(3 \mathrm{H}, \mathrm{s}, \mathrm{OCH}_{2} \mathrm{OCH}_{3}\right), 2.71(1 \mathrm{H}, \mathrm{d}, J=18.3 \mathrm{~Hz}, \mathrm{H} 9 \mathrm{~b}), 1.77$ $(3 \mathrm{H}, \mathrm{d}, J=1.2 \mathrm{~Hz}, \mathrm{H} 15), 1.50$ (3H, s, H12), 1.14 (9H, s, $t$-Bu of TBDPS), 0.79 (9H, s, $t$-Bu of TBS), $0.13\left(3 \mathrm{H}, \mathrm{s}, \mathrm{CH}_{3}\right.$ of TBS), -0.15 (3H, s, $\mathrm{CH}_{3}$ of TBS). ${ }^{13} \mathrm{C}\left\{{ }^{1} \mathrm{H}\right\} \mathrm{NMR}\left(125 \mathrm{MHz}, \mathrm{C}_{6} \mathrm{D}_{6}\right): \delta 202.6,178.1$, 140.2, 136.1 (2C), 136.0 (2C), 133.62, 133.57, 130.10, 130.05, 128.7, 128.5 (4C), 96.0, 88.4, 86.3, 82.3, 78.1, 77.6, 69.1, 62.7, 56.7, 55.7, 40.2, 27.0 (3C), 25.6 (3C), 20.8, 19.5, 19.4, 18.0, -5.0, -5.1. HRMS (ESI-TOF) $[\mathrm{M}+\mathrm{Na}]^{+} \mathrm{m} / \mathrm{z}$ : Calcd for $\mathrm{C}_{39} \mathrm{H}_{54} \mathrm{O}_{8} \mathrm{Si}_{2} \mathrm{Na} 729.3249$; Found 729.3421.

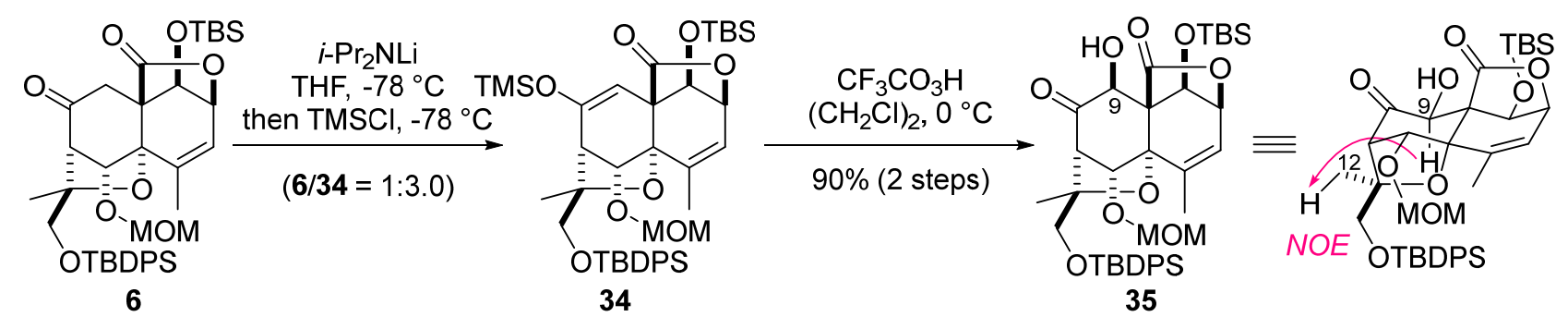

Hydroxy Ketone 35. $n$-BuLi (2.64 M hexane solution, $1.00 \mathrm{~mL}, 2.64 \mathrm{mmol}$ ) was added to a solution of $i-\operatorname{Pr}_{2} \mathrm{NH}(390 \mu \mathrm{L}, 2.78 \mathrm{mmol})$ in THF $(1.20 \mathrm{~mL})$ at $-78^{\circ} \mathrm{C}$. The mixture was stirred at $0{ }^{\circ} \mathrm{C}$ for 30 min, and then cooled to $-78^{\circ} \mathrm{C}$ to give $i-\operatorname{Pr} 2 \mathrm{NLi}(1.0 \mathrm{M}$ THF/hexane solution), which was used directly in the next reaction.

$i$-Pr2 $\mathrm{NLi}$ (1.0 M THF/hexane solution, $2.59 \mathrm{~mL}, 2.64 \mathrm{mmol})$ was added to a solution of A-ring 6 (193 $\mathrm{mg}, 273 \mu \mathrm{mol})$ and chlorotrimethylsilane (TMSCl, $380 \mu \mathrm{L}, 3.00 \mathrm{mmol})$ in THF $(14.0 \mathrm{~mL})$ at $-78^{\circ} \mathrm{C}$ via cannula. After the reaction mixture was stirred at $-78^{\circ} \mathrm{C}$ for $3 \mathrm{~h}, \mathrm{pH} 7$ phosphate buffer $(14 \mathrm{~mL})$ was added to the mixture. The resultant mixture was extracted with EtOAc $(15 \mathrm{~mL} \mathrm{x3})$. The combined organic layers were dried over $\mathrm{Na}_{2} \mathrm{SO}_{4}$, filtered, and concentrated. The residue was passed through a pad of florisil ( $5 \mathrm{~g}$, hexane/EtOAc $=5 / 1)$ to afford a crude silyl enol ether $34(215 \mathrm{mg})$, which was used in the next reaction without further purification. HRMS (ESI-TOF) $[\mathrm{M}+\mathrm{Na}]^{+} \mathrm{m} / \mathrm{z}$ : Calcd for $\mathrm{C}_{42} \mathrm{H}_{62} \mathrm{O}_{8} \mathrm{Si}_{3} \mathrm{Na}$ 801.3645; Found 801.3662.

Trifluoroacetic anhydride $(770 \mu \mathrm{L}, 5.54 \mathrm{mmol})$ was added to a suspension of urea hydrogen peroxide $(470 \mathrm{mg}, 5.00 \mathrm{mmol})$ in 1,2-dichloroethane $(5.00 \mathrm{~mL})$ at $0^{\circ} \mathrm{C}$. After the mixture was vigorously stirred at $0{ }^{\circ} \mathrm{C}$ for $1 \mathrm{~h}$ and at $25^{\circ} \mathrm{C}$ for $1 \mathrm{~h}$, the stirring was stopped. The supernatant $\mathrm{CF}_{3} \mathrm{CO}_{3} \mathrm{H}$ (ca. $1 \mathrm{M} \mathrm{1,2-}$ dichloroethane solution) was directly used in the next reaction. 
The above freshly prepared $\mathrm{CF}_{3} \mathrm{CO}_{3} \mathrm{H}$ (ca. $1 \mathrm{M}$ 1,2-dichloroethane solution, $2.70 \mathrm{~mL}, 2.70 \mathrm{mmol}$ ) was added to a solution of the above crude silyl enol ether $\mathbf{3 4}(215 \mathrm{mg})$ in 1,2-dichloroethane $(5.50 \mathrm{~mL})$ at $0{ }^{\circ} \mathrm{C}$. After the reaction mixture was stirred at $0{ }^{\circ} \mathrm{C}$ for $10 \mathrm{~min}$, saturated aqueous $\mathrm{NaHCO}_{3}(6 \mathrm{~mL})$ and saturated aqueous $\mathrm{Na}_{2} \mathrm{~S}_{2} \mathrm{O}_{3}(6 \mathrm{~mL})$ were successively added to the mixture. The resultant mixture was extracted with $\mathrm{CH}_{2} \mathrm{Cl}_{2}\left(12 \mathrm{~mL}\right.$ x5). The combined organic layers were dried over $\mathrm{Na}_{2} \mathrm{SO}_{4}$, filtered, and concentrated. The residue was purified by automated flash column chromatography using the prepacked silica gel (40 g, hexane/EtOAc $=94 / 6$ to 73/27) to afford hydroxyl ketone 35 (177 mg, 245 $\mu \mathrm{mol}$ ) in $90 \%$ yields over 2 steps. The C9-stereochemistry of $\mathbf{3 5}$ was determined by the NOE experiment (Page S91): white amorphous. $[\alpha]_{\mathrm{D}}^{22}+37.2$ ( $\left.c 0.875, \mathrm{CHCl}_{3}\right)$. IR (film): 3518, 3067, 2936, 2860, 1773, 1730, 1466, 1256, 1196, $1106 \mathrm{~cm}^{-1} .{ }^{1} \mathrm{H}$ NMR (500 MHz, C6 $\left.\mathrm{D}_{6}\right): \delta$ 7.69-7.66 (4H, m, aromatic), 7.20-7.16 (6H, m, aromatic), $5.56(1 \mathrm{H}, \mathrm{dd}, J=6.3,1.2 \mathrm{~Hz}, \mathrm{H} 3), 5.04(1 \mathrm{H}, \mathrm{s}, \mathrm{H} 6), 4.73(1 \mathrm{H}$, d, $J=5.7 \mathrm{~Hz}, \mathrm{H} 9), 4.68$ (1H, s, H1), 4.26 (1H, d, $J=9.2 \mathrm{~Hz}, \mathrm{H} 13 \mathrm{a}), 4.18$ (1H, d, $J=6.3 \mathrm{~Hz}, \mathrm{H} 2), 4.09$ $\left(1 \mathrm{H}, \mathrm{d}, J=6.3 \mathrm{~Hz}, \mathrm{OCHaHbOCH}_{3}\right), 4.04\left(1 \mathrm{H}, \mathrm{d}, J=6.3 \mathrm{~Hz}, \mathrm{OCHaHbOCH}_{3}\right), 3.68(1 \mathrm{H}, \mathrm{s}, \mathrm{H} 7), 3.67$ $(1 \mathrm{H}, \mathrm{d}, J=9.2 \mathrm{~Hz}, \mathrm{H} 13 \mathrm{~b}), 3.54(1 \mathrm{H}, \mathrm{d}, J=5.7 \mathrm{~Hz}, \mathrm{OH}), 2.91\left(3 \mathrm{H}, \mathrm{s}, \mathrm{OCH}_{2} \mathrm{OCH}_{3}\right), 1.68(3 \mathrm{H}, \mathrm{d}, J=$ $1.2 \mathrm{~Hz}, \mathrm{H} 15), 1.36$ (3H, s, H12), 1.11 (9H, s, $t$-Bu of TBDPS), 0.97 (9H, s, $t$-Bu of TBS), 0.09 (3H, s, $\mathrm{CH}_{3}$ of TBS), $-0.02\left(3 \mathrm{H}, \mathrm{s}, \mathrm{CH}_{3}\right.$ of TBS). ${ }^{13} \mathrm{C}\left\{{ }^{1} \mathrm{H}\right\} \mathrm{NMR}\left(125 \mathrm{MHz}, \mathrm{C}_{6} \mathrm{D}_{6}\right): \delta 203.9,173.9,139.6$, 136.1 (2C), 136.0 (2C), 133.5, 133.4, 130.14, 130.09, 129.3, 128 (4C, deduced from HMQC), 96.0, 89.3, 86.2, 83.2, 79.3, 78.1, 74.0, 68.9, 62.1, 61.2, 55.7, 27.0 (3C), 25.8 (3C), 20.0, 19.4, 19.1, 18.2, 4.85, -4.92. HRMS (ESI-TOF) $[\mathrm{M}+\mathrm{Na}]^{+} \mathrm{m} / \mathrm{z}$ : Calcd for $\mathrm{C}_{39} \mathrm{H}_{54} \mathrm{O}_{9} \mathrm{Si}_{2} \mathrm{Na}$ 745.3199; Found 745.3191.

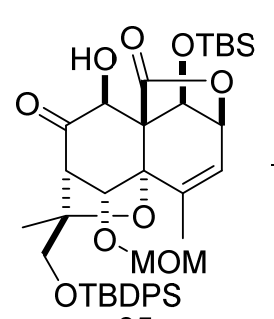

35

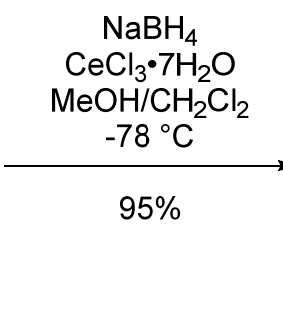

$\mathrm{NaBH}_{4}$ $\mathrm{CeCl}_{3} \cdot 7 \mathrm{H}_{2} \mathrm{O}$ $-78{ }^{\circ} \mathrm{C}$ $95 \%$

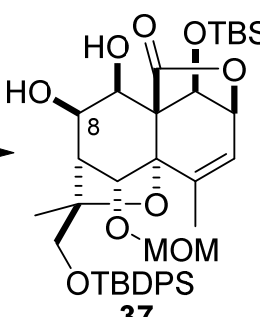

37

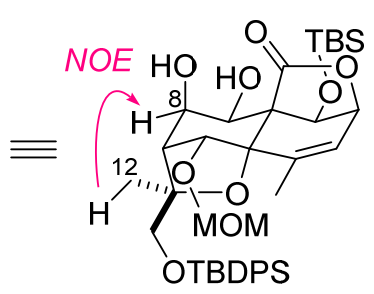

OTBDPS

Diol 37. $\mathrm{NaBH}_{4}(47.7 \mathrm{mg}, 1.26 \mathrm{mmol})$ was added to a solution of hydroxyl ketone 35 (177 mg, 245 $\mu \mathrm{mol})$ and $\mathrm{CeCl}_{3} \cdot 7 \mathrm{H}_{2} \mathrm{O}(187 \mathrm{mg}, 501 \mu \mathrm{mol})$ in $\mathrm{MeOH}(4.50 \mathrm{~mL})$ and $\mathrm{CH}_{2} \mathrm{Cl}_{2}(450 \mu \mathrm{L})$ at $-78^{\circ} \mathrm{C}$. After the reaction mixture was stirred at $-78^{\circ} \mathrm{C}$ for $15 \mathrm{~min}$, saturated aqueous potassium sodium tartrate ( 9 $\mathrm{mL})$ was added to the mixture. After $\mathrm{H}_{2} \mathrm{O}(9 \mathrm{~mL})$ was added to the mixture, the resultant mixture was extracted with EtOAc (18 mL x4). The combined organic layers were dried over $\mathrm{Na}_{2} \mathrm{SO}_{4}$, filtered, and concentrated. The residue was purified by flash column chromatography on silica gel (7.5 g, hexane/EtOAc $=10 / 1$ to $5 / 1)$ to afford diol $37(169 \mathrm{mg}, 233 \mu \mathrm{mol})$ in 95\% yield. The C8stereochemistry of $\mathbf{3 7}$ was determined by the NOE experiment (Page S93): colorless oil. $[\alpha]_{\mathrm{D}^{22}}-3.44$ (c $\left.0.715, \mathrm{CHCl}_{3}\right)$. IR (film) 3529, 2935, 2859, 1766, 1466, 1257, 1104, 1023, $831 \mathrm{~cm}^{-1}$. ${ }^{1} \mathrm{H}$ NMR (500 
$\left.\mathrm{MHz}, \mathrm{CO}\left(\mathrm{CD}_{3}\right)_{2}\right) \delta$ 7.72-7.69 (4H, m, aromatic), 7.49-7.43 (6H, m, aromatic), $6.10(1 \mathrm{H}, \mathrm{dd}, J=6.9$, $1.2 \mathrm{~Hz}, \mathrm{H} 3), 5.00(1 \mathrm{H}, \mathrm{s}, \mathrm{H} 6), 4.62(1 \mathrm{H}, \mathrm{s}, \mathrm{H} 1), 4.61(1 \mathrm{H}, \mathrm{d}, J=6.9 \mathrm{~Hz}, \mathrm{H} 2), 4.56(1 \mathrm{H}, \mathrm{d}, J=6.3 \mathrm{~Hz}$, $\left.\mathrm{OCHaHbOCH}_{3}\right), 4.37(1 \mathrm{H}, \mathrm{dd}, J=5.2,4.0 \mathrm{~Hz}, \mathrm{H} 9), 4.35\left(1 \mathrm{H}, \mathrm{d}, J=6.3 \mathrm{~Hz}, \mathrm{OCHaHbOCH}_{3}\right), 4.32$ $(1 \mathrm{H}, \mathrm{d}, J=9.2 \mathrm{~Hz}, \mathrm{H} 13 \mathrm{a}), 4.29(1 \mathrm{H}, \mathrm{ddd}, J=5.2,4.6,4.0 \mathrm{~Hz}, \mathrm{H} 8), 4.17(1 \mathrm{H}, \mathrm{d}, J=4.6 \mathrm{~Hz}, \mathrm{OH}), 3.46$ $(1 \mathrm{H}, \mathrm{d}, J=9.2 \mathrm{~Hz}, \mathrm{H} 13 \mathrm{~b}), 3.27(1 \mathrm{H}, \mathrm{d}, J=4.0 \mathrm{~Hz}, \mathrm{OH}), 3.22\left(3 \mathrm{H}, \mathrm{s}, \mathrm{OCH}_{2} \mathrm{OCH}_{3}\right), 2.85(1 \mathrm{H}, \mathrm{d}, J=$ $4.0 \mathrm{~Hz}, \mathrm{H} 7), 1.73$ (3H, s, H15), 1.49 (3H, s, H12), 1.08 (9H, s, $t$-Bu of TBDPS), 0.93 (9H, s, $t$-Bu of TBS), 0.22 (3H, s, $\mathrm{CH}_{3}$ of TBS), 0.21 (3H, s, CH3 of TBS). ${ }^{13} \mathrm{C}\left\{{ }^{1} \mathrm{H}\right\} \mathrm{NMR}\left(125 \mathrm{MHz}, \mathrm{CO}\left(\mathrm{CD}_{3}\right)_{2}\right) \delta$ 176.4, 141.0, 136.65 (2C), 136.62 (2C), 134.34, 134.32, 130.88, 130.86, 129.1, 128.8 (4C), 96.1, 87.6, 86.1, 81.8, 81.0, 79.0, 71.1, 70.3, 68.1, 64.7, 56.0, 52.0, 27.4 (3C), 26.2 (3C), 20.0, 19.8, 19.4, 18.8, 4.5, -4.7. HRMS (ESI-TOF) $[\mathrm{M}+\mathrm{Na}]^{+} \mathrm{m} / \mathrm{z}$ : Calcd for $\mathrm{C}_{39} \mathrm{H}_{56} \mathrm{O}_{9} \mathrm{Si}_{2} \mathrm{Na}$ 747.3355; Found 747.3364.
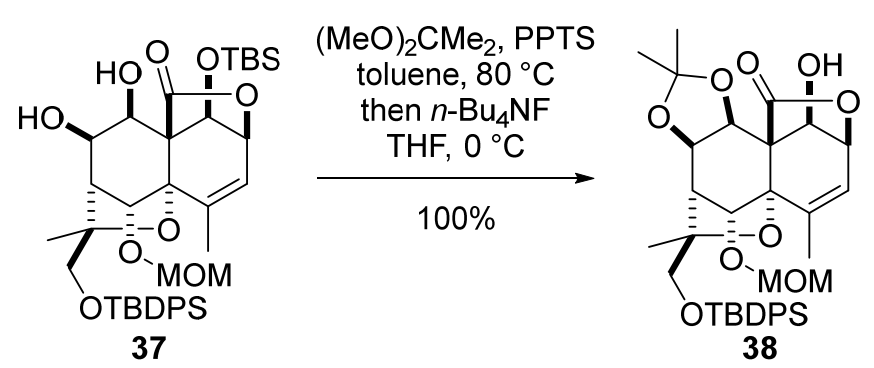

Alcohol 38. Pyridinium p-toluenesulfonate (PPTS, $6.7 \mathrm{mg}, 27 \mu \mathrm{mol}$ ) was added to a solution of diol $37(200 \mathrm{mg}, 276 \mu \mathrm{mol})$ and 2,2-dimethoxypropane $(170 \mu \mathrm{L}, 1.39 \mathrm{mmol})$ in toluene $(5.50 \mathrm{~mL})$ at $25^{\circ} \mathrm{C}$. After the reaction mixture was stirred at $80^{\circ} \mathrm{C}$ for $2 \mathrm{~h}, n$-Bu4NF (1 M THF solution, $2.80 \mathrm{~mL}, 2.80$ mmol) was added dropwise to the mixture at $0{ }^{\circ} \mathrm{C}$. After the reaction mixture was stirred at $0{ }^{\circ} \mathrm{C}$ for $45 \mathrm{~min}$, saturated aqueous $\mathrm{NH}_{4} \mathrm{Cl}(10 \mathrm{~mL})$ was added to the mixture. The resultant mixture was extracted with EtOAc $(10 \mathrm{~mL} x 4)$. The combined organic layers were dried over $\mathrm{Na}_{2} \mathrm{SO}_{4}$, filtered, and concentrated. The residue was purified by flash column chromatography on silica gel (7.5 g, hexane/EtOAc $=10 / 1$ to $1 / 1)$ to afford alcohol $38(179 \mathrm{mg}, 275 \mu \mathrm{mol})$ in $100 \%$ yield: white amorphous. $[\alpha]_{\mathrm{D}}^{25}-3.09$ (c 0.925, $\mathrm{CHCl}_{3}$ ). IR (film): 3441, 2934, 1778, 1466, 1379, 1259, 1212, 1106, $915 \mathrm{~cm}^{-1}$. ${ }^{1} \mathrm{H}$ NMR (500 MHz, $\mathrm{CDCl}_{3}$ ): $\delta$ 7.65-7.62 (4H, m, aromatic), 7.44-7.35 (6H, m, aromatic), $5.95(1 \mathrm{H}$, $\mathrm{dd}, J=6.3,1.2 \mathrm{~Hz}, \mathrm{H} 3), 5.18(1 \mathrm{H}, \mathrm{s}, \mathrm{H} 6), 4.71(1 \mathrm{H}, \mathrm{dd}, J=6.9,2.9 \mathrm{~Hz}, \mathrm{H} 8), 4.54(1 \mathrm{H}, \mathrm{d}, J=6.3 \mathrm{~Hz}$, $\left.\mathrm{OCHaHbOCH}_{3}\right), 4.51(1 \mathrm{H}, \mathrm{d}, J=6.9 \mathrm{~Hz}, \mathrm{H} 9), 4.49(1 \mathrm{H}, \mathrm{d}, J=6.3 \mathrm{~Hz}, \mathrm{H} 2), 4.43(1 \mathrm{H}, \mathrm{s}, \mathrm{H} 1), 4.39$ $\left(1 \mathrm{H}, \mathrm{d}, J=6.3 \mathrm{~Hz}, \mathrm{OCHaHbOCH}_{3}\right), 4.29(1 \mathrm{H}, \mathrm{d}, J=9.2 \mathrm{~Hz}, \mathrm{H} 13 \mathrm{a}), 3.42(1 \mathrm{H}, \mathrm{d}, J=9.2 \mathrm{~Hz}, \mathrm{H} 13 \mathrm{~b})$, $3.26\left(3 \mathrm{H}, \mathrm{s}, \mathrm{OCH}_{2} \mathrm{OCH}_{3}\right), 3.05(1 \mathrm{H}, \mathrm{d}, J=2.9 \mathrm{~Hz}, \mathrm{H} 7), 1.70(3 \mathrm{H}, \mathrm{s}, \mathrm{CH} 3$ of acetonide), 1.69 (3H, d, $J$ $=1.2 \mathrm{~Hz}, \mathrm{H} 15), 1.44(3 \mathrm{H}, \mathrm{s}, \mathrm{H} 12), 1.41(3 \mathrm{H}, \mathrm{s}, \mathrm{CH} 3$ of acetonide), 1.07 (9H, s, $t$-Bu of TBDPS). ${ }^{13} \mathrm{C}\left\{{ }^{1} \mathrm{H}\right\} \operatorname{NMR}\left(125 \mathrm{MHz}, \mathrm{CDCl}_{3}\right): \delta 172.5,140.1,135.7$ (2C), 135.6 (2C), 133.3, 133.2, 129.74, 129.70, 127.9, 127.64 (2C), 127.61 (2C), 111.8, 95.8, 87.2, 85.6, 80.8, 76.8, 76.5, 76.3, 72.3, 68.9, 
63.6, 56.0, 46.1, 26.9 (3C), 25.3, 24.9, 21.2, 19.2, 19.0. HRMS (ESI-TOF) $[\mathrm{M}+\mathrm{Na}]^{+} \mathrm{m} / \mathrm{z}$ : Calcd for $\mathrm{C}_{36} \mathrm{H}_{46} \mathrm{O} 9 \mathrm{SiNa}$ 673.2803; Found 673.2833.

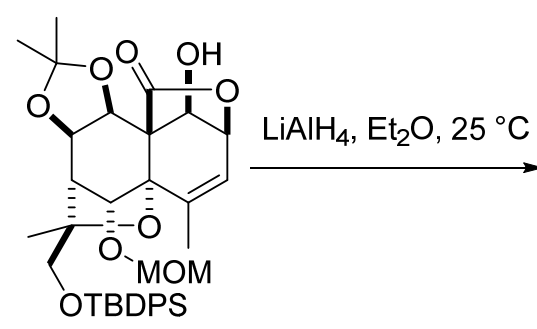

38

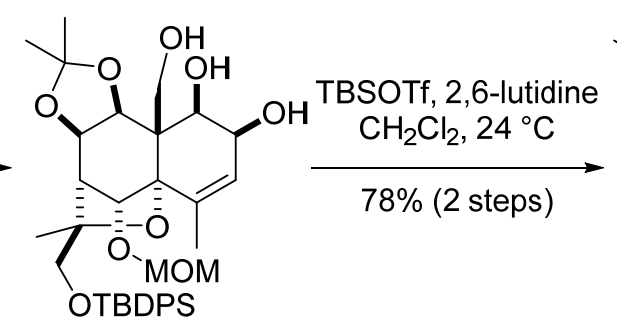

39

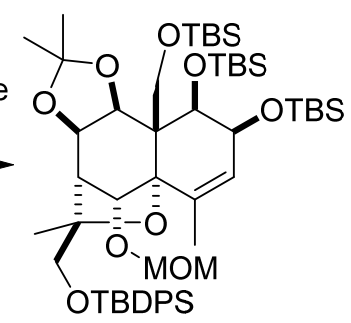

40

Tris TBS-ether 40. $\mathrm{LiAlH}_{4}(1 \mathrm{M} \mathrm{Et} 2 \mathrm{O}$ solution, $1.70 \mathrm{~mL}, 1.70 \mathrm{mmol})$ was added to a solution of alcohol $38(114 \mathrm{mg}, 175 \mu \mathrm{mol})$ in $\mathrm{Et}_{2} \mathrm{O}(17.0 \mathrm{~mL})$ at $25^{\circ} \mathrm{C}$. After the reaction mixture was stirred at $25{ }^{\circ} \mathrm{C}$ for $1 \mathrm{~h}$, saturated aqueous potassium sodium tartrate $(20 \mathrm{~mL})$ and EtOAc $(15 \mathrm{~mL})$ were successively added to the mixture at $0{ }^{\circ} \mathrm{C}$. After the mixture was vigorously stirred at $25^{\circ} \mathrm{C}$ for $12 \mathrm{~h}$, the resultant mixture was extracted with EtOAc $(20 \mathrm{~mL} \mathrm{x8})$. The combined organic layers were dried over $\mathrm{Na}_{2} \mathrm{SO}_{4}$, filtered, and concentrated to afford the crude triol 39 (114 mg), which was used in the next reaction without further purification: ${ }^{1} \mathrm{H} \mathrm{NMR}\left(400 \mathrm{MHz}, \mathrm{CDCl}_{3}\right): \delta 7.67-7.59(4 \mathrm{H}, \mathrm{m}$, aromatic), 7.45-7.32 (6H, m, aromatic), $5.73(1 \mathrm{H}, \mathrm{s}, \mathrm{H} 3), 4.67(1 \mathrm{H}, \mathrm{dd}, J=6.8,3.2 \mathrm{~Hz}, \mathrm{H} 8), 4.60(1 \mathrm{H}, \mathrm{d}, J=6.8$ $\mathrm{Hz}, \mathrm{H} 9), 4.55\left(1 \mathrm{H}, \mathrm{d}, J=6.9 \mathrm{~Hz}, \mathrm{OCHaHbOCH}_{3}\right), 4.50(1 \mathrm{H}, \mathrm{s}, \mathrm{H} 6), 4.33(1 \mathrm{H}, \mathrm{d}, J=6.9 \mathrm{~Hz}$, $\left.\mathrm{OCHaHbOCH}_{3}\right), 4.28$ (1H, d, J = 9.2 Hz, H13a), 4.21 (2H, s, H1, H2), 4.03 (1H, d, J=12.4 Hz, H14a), $3.97(1 \mathrm{H}, \mathrm{d}, J=12.4 \mathrm{~Hz}, \mathrm{H} 14 \mathrm{~b}), 3.34(1 \mathrm{H}, \mathrm{d}, J=9.2 \mathrm{~Hz}, \mathrm{H} 13 \mathrm{~b}), 3.29\left(3 \mathrm{H}, \mathrm{s}, \mathrm{OCH}_{2} \mathrm{OCH}_{3}\right), 3.06(1 \mathrm{H}$, d, $J=3.2 \mathrm{~Hz}, \mathrm{H} 7), 1.79$ (3H, s, H15), $1.64\left(3 \mathrm{H}, \mathrm{s}, \mathrm{CH}_{3}\right.$ of acetonide), 1.48 (3H, s, $\mathrm{CH}_{3}$ of acetonide), $1.39(3 \mathrm{H}, \mathrm{s}, \mathrm{H} 12), 1.06$ (9H, s, $t$-Bu of TBDPS). ${ }^{13} \mathrm{C}\left\{{ }^{1} \mathrm{H}\right\} \mathrm{NMR}\left(125 \mathrm{MHz}, \mathrm{CDCl}_{3}\right): \delta 136.4,135.7$ (2C), 135.6 (2C), 133.4, 133.1, 129.73, 129.67, 129.2, 127.62 (2C), 127.58 (2C), 109.8, 95.1, 87.8, 83.0, 78.9, 78.2, 77.3, 73.2, 69.2, 66.7, 65.4, 56.1, 51.9, 46.6, 26.9 (3C), 25.9, 24.9, 21.6, 21.3, 19.2. HRMS (ESI-TOF) $[\mathrm{M}+\mathrm{Na}]^{+} \mathrm{m} / \mathrm{z}$ : Calcd for $\mathrm{C}_{36} \mathrm{H}_{50} \mathrm{O} 9 \mathrm{SiNa}$ 677.3116; Found 677.3123.

TBSOTf $(600 \mu \mathrm{L}, 2.61 \mathrm{mmol})$ was added to a solution of the above crude triol $39(114 \mathrm{mg})$ and 2,6lutidine $(600 \mu \mathrm{L}, 5.21 \mathrm{mmol})$ in $\mathrm{CH}_{2} \mathrm{Cl}_{2}(8.80 \mathrm{~mL})$ at $24{ }^{\circ} \mathrm{C}$. After the reaction mixture was stirred at $24{ }^{\circ} \mathrm{C}$ for $1.5 \mathrm{~h}$, pH 7 phosphate buffer $(10 \mathrm{~mL})$ was added to the mixture. The resultant mixture was extracted with $\mathrm{CH}_{2} \mathrm{Cl}_{2}(10 \mathrm{~mL} \times 3)$. The combined organic layers were dried over $\mathrm{Na}_{2} \mathrm{SO}_{4}$, filtered, and concentrated. The residue was purified by automated flash column chromatography using the prepacked silica gel $(16 \mathrm{~g}$, hexane/EtOAc $=100 / 0$ to 95/5) to afford tris TBS-ether $\mathbf{4 0}(137 \mathrm{mg}, 137$ $\mu \mathrm{mol})$ in $78 \%$ yield over 2 steps: white amorphous. $[\alpha]_{\mathrm{D}}{ }^{24}+35.8$ (c 1.08, $\left.\mathrm{CHCl}_{3}\right)$. IR (film): 2932, 2890, 2857, 1468, 1366, 1255, 1112, 1000, 886, $837 \mathrm{~cm}^{-1} .{ }^{1} \mathrm{H}$ NMR (400 MHz, $\left.\mathrm{CDCl}_{3}\right): \delta$ 7.67-7.60 (4H, m, aromatic), 7.43-7.31 (6H, m, aromatic), $5.54(1 \mathrm{H}, \mathrm{dd}, J=3.2,1.4 \mathrm{~Hz}, \mathrm{H} 3), 5.46$ (1H, s, H6), 4.59-4.54 $\left(3 \mathrm{H}, \mathrm{m}, \mathrm{H} 1, \mathrm{OCH}_{2} \mathrm{OCH}_{3}\right), 4.33(1 \mathrm{H}, \mathrm{d}, J=9.2 \mathrm{~Hz}, \mathrm{H} 13 \mathrm{a}), 4.21-4.17$ (3H, m, H2, H8, H9), 4.13 (1H, 
d, $J=11.4 \mathrm{~Hz}, \mathrm{H} 14 \mathrm{a}), 4.04(1 \mathrm{H}, \mathrm{d}, J=11.4 \mathrm{~Hz}, \mathrm{H} 14 \mathrm{~b}), 3.36(1 \mathrm{H}, \mathrm{d}, J=9.2 \mathrm{~Hz}, \mathrm{H} 13 \mathrm{~b}), 3.27$ (3H, s, $\left.\mathrm{OCH}_{2} \mathrm{OCH}_{3}\right), 2.96(1 \mathrm{H}, \mathrm{d}, J=2.3 \mathrm{~Hz}, \mathrm{H} 7), 1.85$ (3H, s, H15), 1.56 (3H, s, $\mathrm{CH}_{3}$ of acetonide), 1.314 (3H, s, $\mathrm{CH}_{3}$ of acetonide), 1.306 (3H, s, H12), 1.05 (9H, s, $t$-Bu of TBDPS), 0.92 (9H, s, $t$-Bu of TBS), 0.89 (9H, s, $t$-Bu of TBS), 0.88 (9H, s, $t$-Bu of TBS), 0.079 (3H, s, CH3 of TBS), 0.075 (6H, s, CH3 of TBS x2), 0.05 (3H, s, CH3 of TBS), $0.02\left(6 \mathrm{H}, \mathrm{s}, \mathrm{CH}\right.$ of TBS x2). ${ }^{13} \mathrm{C}\left\{{ }^{1} \mathrm{H}\right\}$ NMR $\left(125 \mathrm{MHz}, \mathrm{CDCl}_{3}\right)$ : $\delta 135.9$ (2C), 135.8 (2C), 135.2, 133.7, 133.5, 130.0, 129.6, 129.5, 127.5 (4C), 108.1, 96.7, 89.1, 81.5, 81.3, 76.2, 75.1, 72.6, 69.8, 67.7, 62.5, 56.3, 54.4, 47.4, 27.0 (6C), 26.5 (3C), 26.3, 26.2 (3C), 23.8, 22.5, 22.0, 19.3, 19.1, 18.4, 18.0, -2.4, -2.6, -4.5 (2C), -4.8, -5.2. HRMS (ESI-TOF) $[\mathrm{M}+\mathrm{Na}]^{+} \mathrm{m} / \mathrm{z}$ : Calcd for $\mathrm{C}_{54} \mathrm{H}_{92} \mathrm{O}_{9} \mathrm{Si}_{4} \mathrm{Na}$ 1019.5711; Found 1019.5726.
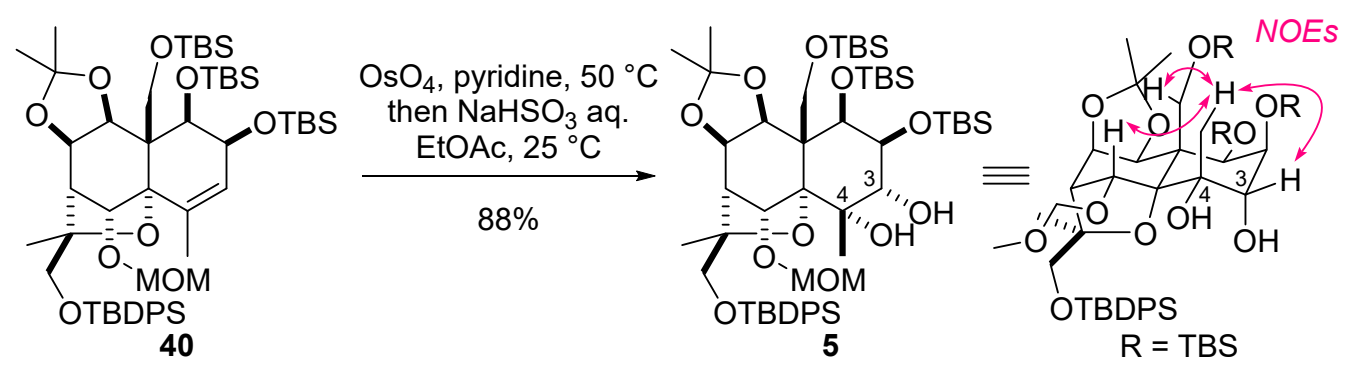

Protected euonyminol 5. $\mathrm{OsO}_{4}(0.49 \mathrm{M}$ pyridine solution, $1.80 \mathrm{~mL}, 882 \mu \mathrm{mol})$ was added to a solution of tris TBS-ether $\mathbf{4 0}(171 \mathrm{mg}, 172 \mu \mathrm{mol})$ in pyridine $(8.50 \mathrm{~mL})$ at $24{ }^{\circ} \mathrm{C}$. After the reaction mixture was stirred at $50{ }^{\circ} \mathrm{C}$ for $25 \mathrm{~h}$, saturated aqueous $\mathrm{NaHSO}_{3}(35.0 \mathrm{~mL})$ and EtOAc $(8.50 \mathrm{~mL})$ were successively added to the mixture at $25^{\circ} \mathrm{C}$. After the reaction mixture was stirred at $25^{\circ} \mathrm{C}$ for $24 \mathrm{~h}$, the resultant mixture was extracted with EtOAc $(40 \mathrm{~mL} \mathrm{x} 4)$. The combined organic layers were dried over $\mathrm{Na}_{2} \mathrm{SO}_{4}$, filtered, and concentrated. The residue was purified by flash column chromatography on silica gel $(7.5 \mathrm{~g}$, hexane/EtOAc $=50 / 1$ to $15 / 1)$ to afford protected euonyminol 5 (156 mg, $151 \mu \mathrm{mol})$ in $88 \%$ yield. The C3,4-stereochemistries of 5 were determined by the NOESY experiment (Page S98): white amorphous. $[\alpha]_{\mathrm{D}}{ }^{25}-15.3$ ( $c$ 1.00, $\mathrm{CHCl}_{3}$ ). IR (film): 3527, 2933, 2858, 1468, 1367, 1256, 1109, 1004, 883, $837 \mathrm{~cm}^{-1} .{ }^{1} \mathrm{H}$ NMR (400 MHz, $\left.\mathrm{CDCl}_{3}\right): \delta$ 7.66-7.64 (4H, m, aromatic), 7.44-7.33 (6H, m, aromatic), $5.54(1 \mathrm{H}, \mathrm{s}, \mathrm{H} 6), 4.68(1 \mathrm{H}, \mathrm{d}, J=12.4 \mathrm{~Hz}, \mathrm{H} 14 \mathrm{a}), 4.57-4.51$ $\left(3 \mathrm{H}, \mathrm{m}, \mathrm{H} 8, \mathrm{OCH}_{2} \mathrm{OCH}_{3}\right), 4.46(1 \mathrm{H}, \mathrm{d}, J=7.8 \mathrm{~Hz}, \mathrm{OH}), 4.19(1 \mathrm{H}, \mathrm{d}, J=4.1 \mathrm{~Hz}, \mathrm{H} 1), 4.11(1 \mathrm{H}, \mathrm{d}, J=$ $12.4 \mathrm{~Hz}, \mathrm{H} 14 \mathrm{~b}), 4.05$ (1H, dd, $J=4.1,2.3 \mathrm{~Hz}, \mathrm{H} 2), 3.94(1 \mathrm{H}, \mathrm{d}, J=7.8 \mathrm{~Hz}, \mathrm{H} 9), 3.92$ (1H, d, $J=10.0$ Hz, H13a), 3.82 (1H, d, $J=10.0 \mathrm{~Hz}, \mathrm{H} 13 \mathrm{~b}), 3.77(1 \mathrm{H}, \mathrm{s}, \mathrm{OH}), 3.61(1 \mathrm{H}, \mathrm{dd}, J=7.8,2.3 \mathrm{~Hz}, \mathrm{H} 3), 3.07$ (3H, s, $\left.\mathrm{OCH}_{2} \mathrm{OCH}_{3}\right), 2.72(1 \mathrm{H}, \mathrm{d}, J=2.3 \mathrm{~Hz}, \mathrm{H} 7), 1.64(3 \mathrm{H}, \mathrm{s}, \mathrm{H} 15), 1.53$ (3H, s, CH3 of acetonide), $1.37(3 \mathrm{H}, \mathrm{s}, \mathrm{H} 12), 1.29\left(3 \mathrm{H}, \mathrm{s}, \mathrm{CH}_{3}\right.$ of acetonide), $1.08(9 \mathrm{H}, \mathrm{s}, t$-Bu of TBDPS), $0.920(18 \mathrm{H}, \mathrm{s}, t$-Bu of TBS x2), 0.917 (9H, s, $t$-Bu of TBS), 0.15 (3H, s, CH3 of TBS), 0.13 (3H, s, CH3 of TBS), 0.11 (3H, s, $\mathrm{CH}_{3}$ of TBS), 0.09 (3H, s, CH3 of TBS), 0.07 (3H, s, $\mathrm{CH}_{3}$ of TBS), $0.04\left(3 \mathrm{H}, \mathrm{s}, \mathrm{CH}_{3}\right.$ of TBS). ${ }^{13} \mathrm{C}\left\{{ }^{1} \mathrm{H}\right\}$ NMR (125 MHz, $\mathrm{CDCl}_{3}$ ): $\delta 135.9$ (2C), 135.8 (2C), 133.4, 133.2, 129.7 (2C), 127.6 (4C), 108.4, 97.2, 
95.5, 84.0, 83.1, 80.0, 77.9 (2C), 75.9, 74.1, 71.5, 68.5, 59.9, 56.5, 55.9, 45.7, 27.0 (6C), 26.9, 26.4 (6C), 25.3, 23.7, 21.2, 19.2, 18.5, 18.4, 18.1, -2.3, -4.06, -4.10, -4.3, -4.4, -4.5. HRMS (ESI-TOF) $[\mathrm{M}+\mathrm{Na}]^{+} \mathrm{m} / \mathrm{z}$ : Calcd for $\mathrm{C}_{54} \mathrm{H}_{94} \mathrm{O}_{11} \mathrm{Si}_{4} \mathrm{Na}$ 1053.5765; Found 1053.5795.

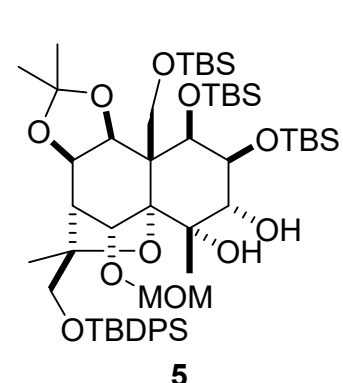

5

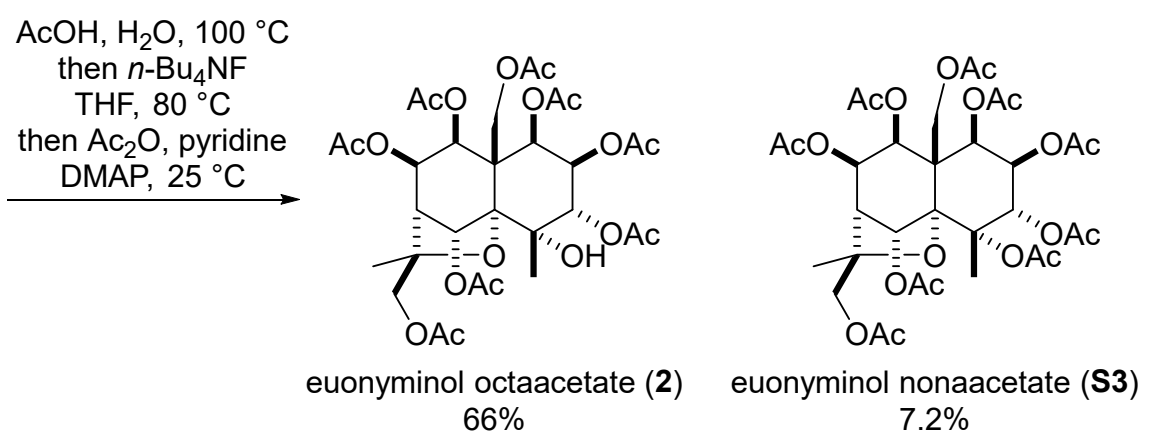

Euonyminol Octaacetate (2) [CAS: 33492-70-5]. A solution of protected euonyminol 5 (10.6 mg, $10.3 \mu \mathrm{mol})$ in $\mathrm{AcOH}(3.90 \mathrm{~mL})$ and $\mathrm{H}_{2} \mathrm{O}(1.30 \mathrm{~mL})$ was stirred at $100{ }^{\circ} \mathrm{C}$ for $12 \mathrm{~h}$. After being cooled to $24{ }^{\circ} \mathrm{C}$, the reaction mixture was diluted with toluene $(6 \mathrm{~mL})$ and concentrated. The residue was dissolved in THF (3.40 mL). $n$-Bu4NF (1 M THF solution, $310 \mu \mathrm{L}, 310 \mu \mathrm{mol}$ ) was added to the solution at $24{ }^{\circ} \mathrm{C}$. After the reaction mixture was stirred at $80{ }^{\circ} \mathrm{C}$ for $3 \mathrm{~h}$, pyridine $(2.20 \mathrm{~mL}), \mathrm{Ac}_{2} \mathrm{O}$ $(1.10 \mathrm{~mL})$, and 4-dimethylaminopyridine (DMAP, $24.2 \mathrm{mg}, 0.198 \mathrm{mmol}$ ) were successively added to the mixture at $25{ }^{\circ} \mathrm{C}$. After the reaction mixture was stirred at $25^{\circ} \mathrm{C}$ for $17 \mathrm{~h}$, saturated aqueous $\mathrm{NaHCO}_{3}(10 \mathrm{~mL})$ was added to the mixture. The resultant mixture was extracted with EtOAc $(10 \mathrm{~mL}$ x6). The combined organic layers were dried over $\mathrm{Na}_{2} \mathrm{SO}_{4}$, filtered, and concentrated. The residue was passed through a pad of silica gel $(2.5 \mathrm{~g}$, hexane/EtOAc $=1 / 1)$ and concentrated. The residue was purified by PTLC $(20 \mathrm{~cm} \times 10 \mathrm{~cm}$, hexane $/ t$-BuOMe $=1 / 4$, developed five times $)$ to afford euonyminol octaacetate (2) $(4.76 \mathrm{mg}, 6.78 \mu \mathrm{mol})$ and euonyminol nonaacetate $(\mathbf{S 3})(0.55 \mathrm{mg}, 0.74 \mu \mathrm{mol})$ in $66 \%$ and $7.2 \%$ yields, respectively:

2: white amorphous. $[\alpha]_{\mathrm{D}}{ }^{28}-10.4\left(c 0.238, \mathrm{CHCl}_{3}\right)$. IR (film): 3461, 2924, 1744, 1370, 1234, $1045 \mathrm{~cm}^{-}$ ${ }^{1} .{ }^{1} \mathrm{H}$ NMR (400 MHz, $\left.\mathrm{CDCl}_{3}\right): \delta 6.77(1 \mathrm{H}, \mathrm{s}, \mathrm{H} 6), 5.58(1 \mathrm{H}, \mathrm{d}, J=3.7 \mathrm{~Hz}, \mathrm{H} 1), 5.48(1 \mathrm{H}, \mathrm{dd}, J=6.0$, $3.7 \mathrm{~Hz}, \mathrm{H} 8), 5.34(1 \mathrm{H}, \mathrm{d}, J=6.0 \mathrm{~Hz}, \mathrm{H} 9), 5.27(1 \mathrm{H}, \mathrm{dd}, J=3.7,2.8 \mathrm{~Hz}, \mathrm{H} 2), 5.20(1 \mathrm{H}, \mathrm{d}, J=13.3 \mathrm{~Hz}$, H14a), 4.90 (1H, d, $J=11.9 \mathrm{~Hz}, \mathrm{H} 13 \mathrm{a}), 4.82(1 \mathrm{H}, \mathrm{d}, J=2.8 \mathrm{~Hz}, \mathrm{H} 3), 4.42$ (1H, d, $J=13.3 \mathrm{~Hz}, \mathrm{H} 14 \mathrm{~b})$, $4.20(1 \mathrm{H}, \mathrm{d}, J=1.4 \mathrm{~Hz}, \mathrm{OH}), 3.93(1 \mathrm{H}, \mathrm{d}, J=11.9 \mathrm{~Hz}, \mathrm{H} 13 \mathrm{~b}), 2.32(1 \mathrm{H}, \mathrm{d}, J=3.7 \mathrm{~Hz}, \mathrm{H} 7), 2.25(3 \mathrm{H}$, s, $\mathrm{CH}_{3}$ of $\left.\mathrm{Ac}\right), 2.164\left(3 \mathrm{H}, \mathrm{s}, \mathrm{CH}_{3}\right.$ of $\left.\mathrm{Ac}\right), 2.158\left(3 \mathrm{H}, \mathrm{s}, \mathrm{CH}_{3}\right.$ of $\left.\mathrm{Ac}\right), 2.13\left(3 \mathrm{H}, \mathrm{s}, \mathrm{CH}_{3}\right.$ of $\left.\mathrm{Ac}\right), 2.12(3 \mathrm{H}$, s, $\mathrm{CH}_{3}$ of Ac), $2.11\left(3 \mathrm{H}, \mathrm{s}, \mathrm{CH}_{3}\right.$ of $\left.\mathrm{Ac}\right), 1.98\left(3 \mathrm{H}, \mathrm{s}, \mathrm{CH}_{3}\right.$ of $\left.\mathrm{Ac}\right), 1.88\left(3 \mathrm{H}, \mathrm{s}, \mathrm{CH}_{3}\right.$ of $\left.\mathrm{Ac}\right), 1.57$ (3H, s, $\mathrm{H} 12), 1.47(3 \mathrm{H}, \mathrm{d}, J=1.4 \mathrm{~Hz}, \mathrm{H} 15) .{ }^{13} \mathrm{C}\left\{{ }^{1} \mathrm{H}\right\} \mathrm{NMR}\left(125 \mathrm{MHz}, \mathrm{CDCl}_{3}\right): \delta 170.8,170.13,170.08,169.8$, 169.6, 169.3, 169.0, 168.7, 92.9, 84.1, 75.8, 73.9, 73.1, 70.9, 69.4, 69.3, 69.0, 68.9, 60.3, 51.9, 50.7, 23.2, 21.5, 21.2, 21.0 (2C), 20.9, 20.8, 20.52, 20.48, 18.3. HRMS (ESI-TOF) $[\mathrm{M}+\mathrm{Na}]^{+} \mathrm{m} / \mathrm{z}$ : Calcd for $\mathrm{C}_{31} \mathrm{H}_{42} \mathrm{O}_{18} \mathrm{Na}$ 725.2263; Found 725.2267. 
S3: colorless oil. $[\alpha]_{\mathrm{D}}^{24}-9.20$ (c 0.049, $\mathrm{CHCl}_{3}$ ). IR (film): 2922, 2851, 1744, 1371, 1235, $1047 \mathrm{~cm}^{-1}$. ${ }^{1} \mathrm{H}$ NMR (400 MHz, CDCl $): \delta 6.91(1 \mathrm{H}, \mathrm{s}, \mathrm{H} 6), 5.68(1 \mathrm{H}, \mathrm{d}, J=4.1 \mathrm{~Hz}, \mathrm{H} 1), 5.67(1 \mathrm{H}, \mathrm{d}, J=2.8 \mathrm{~Hz}$, H3), $5.47(1 \mathrm{H}, \mathrm{dd}, J=6.4,3.7 \mathrm{~Hz}, \mathrm{H} 8), 5.40(1 \mathrm{H}, \mathrm{d}, J=6.4 \mathrm{~Hz}, \mathrm{H} 9), 5.35$ (1H, dd, $J=4.1,2.8 \mathrm{~Hz}$, H2), 5.07 (1H, d, $J=13.8 \mathrm{~Hz}, \mathrm{H} 14 \mathrm{a}), 5.04(1 \mathrm{H}, \mathrm{d}, J=11.5 \mathrm{~Hz}, \mathrm{H} 13 \mathrm{a}), 4.50$ (1H, d, $J=13.8 \mathrm{~Hz}, \mathrm{H} 14 \mathrm{~b})$, $3.76(1 \mathrm{H}, \mathrm{d}, J=11.5 \mathrm{~Hz}, \mathrm{H} 13 \mathrm{~b}), 2.49(1 \mathrm{H}, \mathrm{d}, J=3.7 \mathrm{~Hz}, \mathrm{H} 7), 2.27$ (3H, s, CH3 of Ac), 2.16 (3H, s, $\mathrm{CH}_{3}$ of $\left.\mathrm{Ac}\right), 2.15\left(3 \mathrm{H}, \mathrm{s}, \mathrm{CH}_{3}\right.$ of $\left.\mathrm{Ac}\right), 2.10\left(3 \mathrm{H}, \mathrm{s}, \mathrm{CH}_{3}\right.$ of $\left.\mathrm{Ac}\right), 2.07\left(6 \mathrm{H}, \mathrm{s}, \mathrm{CH}_{3}\right.$ of $\left.\mathrm{Ac} \times 2\right), 2.06(3 \mathrm{H}, \mathrm{s}$, $\mathrm{CH}_{3}$ of $\left.\mathrm{Ac}\right), 1.99$ (3H, s, $\mathrm{CH}_{3}$ of $\left.\mathrm{Ac}\right), 1.87$ (3H, s, $\mathrm{CH}_{3}$ of $\left.\mathrm{Ac}\right), 1.76(3 \mathrm{H}, \mathrm{s}, \mathrm{H} 15), 1.59$ (3H, s, H12). ${ }^{13} \mathrm{C}\left\{{ }^{1} \mathrm{H}\right\}$ NMR $\left(125 \mathrm{MHz}, \mathrm{CDCl}_{3}\right): \delta 170.6,170.09,170.06,169.4,169.1,168.9,168.8(2 \mathrm{C}), 168.4$, 91.6, 82.7, 78.4, 74.6, 73.0, 72.9, 71.1, 69.2, 69.1, 68.1, 59.8, 52.5, 49.0, 21.8, 21.31, 21.28, 21.03, 20.96, 20.9, 20.7, 20.49, 20.45, 20.4, 20.0. [M+Na] ${ }^{+}$HRMS (ESI-TOF) m/z: Calcd for $\mathrm{C}_{33} \mathrm{H}_{44} \mathrm{O}_{19} \mathrm{Na}$ 767.2369; Found 767.2392.

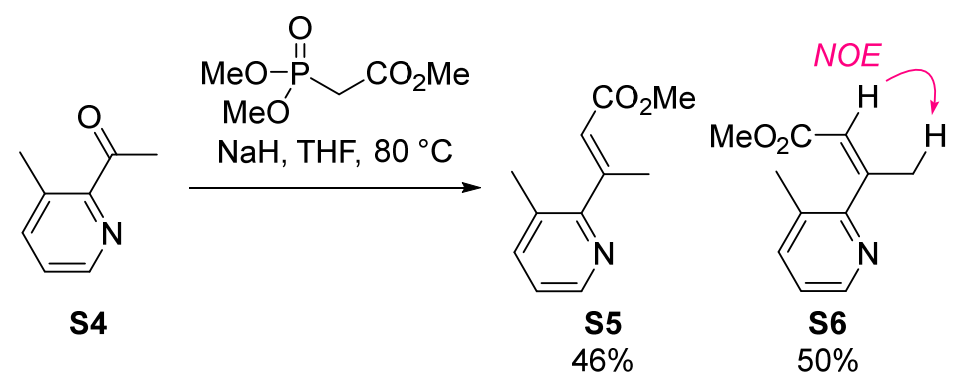

Alkenes S5 and S6. Methyl dimethylphosphonoacetate $(1.70 \mathrm{~mL}, 11.9 \mathrm{mmol})$ was added dropwise to a suspension of $\mathrm{NaH}(50-70 \mathrm{wt} \%$ in mineral oil, $479 \mathrm{mg}, 9.98-14.0 \mathrm{mmol})$ in $\mathrm{THF}(25.0 \mathrm{~mL})$ at $0{ }^{\circ} \mathrm{C}$. After the mixture was stirred at $0{ }^{\circ} \mathrm{C}$ for $30 \mathrm{~min}$, ketone $\mathbf{S 4}(1.30 \mathrm{~mL}, 10.0 \mathrm{mmol})$ was added to the mixture. After the reaction mixture was stirred at $80{ }^{\circ} \mathrm{C}$ for $12 \mathrm{~h}$, saturated aqueous $\mathrm{NH}_{4} \mathrm{Cl}(25 \mathrm{~mL})$ and $\mathrm{H}_{2} \mathrm{O}(10 \mathrm{~mL})$ were successively added to the mixture at $23^{\circ} \mathrm{C}$. The resultant mixture was extracted with EtOAc $(25 \mathrm{~mL} x 3)$. The combined organic layers were washed with brine $(25 \mathrm{~mL})$, dried over $\mathrm{Na}_{2} \mathrm{SO}_{4}$, filtered, and concentrated. The residue was purified by automated flash column chromatography using the prepacked silica gel (40 g, hexane/EtOAc $=73 / 37$ to $52 / 48$ ) to afford alkenes S5 (887 mg, $4.64 \mathrm{mmol}$ ) and S6 (946 mg, $4.95 \mathrm{mmol})$ in 46\% and 50\% yields, respectively. The stereochemistry of S6 was determined by the NOE experiment (Page S103).

S5: colorless oil. IR (film): 2950, 2927, 1720, 1646, 1436, 1284, 1196, $1170 \mathrm{~cm}^{-1} .{ }^{1} \mathrm{H}$ NMR (400 MHz, $\left.\mathrm{CDCl}_{3}\right): \delta 8.36\left(1 \mathrm{H}, \mathrm{d}, J=4.6 \mathrm{~Hz}, \mathrm{H}^{\prime}{ }^{\prime}\right), 7.46\left(1 \mathrm{H}, \mathrm{d}, J=7.8 \mathrm{~Hz}, \mathrm{H} 4{ }^{\prime}\right), 7.08(1 \mathrm{H}, \mathrm{dd}, J=7.8,4.6 \mathrm{~Hz}$, $\mathrm{H}^{\prime}$ '), $5.80\left(1 \mathrm{H}, \mathrm{s}, \mathrm{H} 8\right.$ ') $, 3.68\left(3 \mathrm{H}, \mathrm{s}, \mathrm{CO}_{2} \mathrm{CH}_{3}\right), 2.46\left(3 \mathrm{H}, \mathrm{s}, \mathrm{H} 12\right.$ '), $2.26\left(3 \mathrm{H}, \mathrm{s}, \mathrm{H} 9\right.$ ') ${ }^{13} \mathrm{C}\left\{{ }^{1} \mathrm{H}\right\} \mathrm{NMR}$ $\left(100 \mathrm{MHz}, \mathrm{CDCl}_{3}\right): \delta 166.7,159.8,155.9,146.5,138.4,129.4,122.5,119.9,51.0,19.01,18.95$. HRMS (ESI-TOF) $[\mathrm{M}+\mathrm{Na}]^{+} \mathrm{m} / \mathrm{z}$ : Calcd for $\mathrm{C}_{11} \mathrm{H}_{13} \mathrm{NO}_{2} \mathrm{Na} 214.0839$; Found 214.0848.

S6: colorless oil. IR (film): 2980, 2951, 1727, 1654, 1448, 1254, 1195, 1163, 1045, $793 \mathrm{~cm}^{-1}$. ${ }^{1} \mathrm{H}$ NMR (500 MHz, C6 $\left.\mathrm{D}_{6}\right): \delta 8.41$ (1H, d, $J=5.2 \mathrm{~Hz}, \mathrm{H6}$ ') 7.00 (1H, d, $J=8.0 \mathrm{~Hz}, \mathrm{H} 4$ ') 6.65 (1H, dd, $J=8.0$, 
$5.2 \mathrm{~Hz}, \mathrm{H} 5$ '), 5.96 (1H, s, H8'), 3.19 (3H, s, $\left.\mathrm{CO}_{2} \mathrm{CH}_{3}\right), 2.01$ (3H, s, H12'), 1.90 (3H, s, H9'). $\left.{ }^{13} \mathrm{C}^{1}{ }^{1} \mathrm{H}\right\}$ NMR (100 MHz, $\left.\mathrm{CDCl}_{3}\right): \delta$ 165.2, 158.6, 155.3, 146.3, 137.0, 129.2, 121.9, 118.3, 50.8, 24.7, 17.8 . HRMS (ESI-TOF) $[\mathrm{M}+\mathrm{Na}]^{+} \mathrm{m} / \mathrm{z}$ : Calcd for $\mathrm{C}_{11} \mathrm{H}_{13} \mathrm{NO}_{2} \mathrm{Na}$ 214.0839; Found 214.0847.

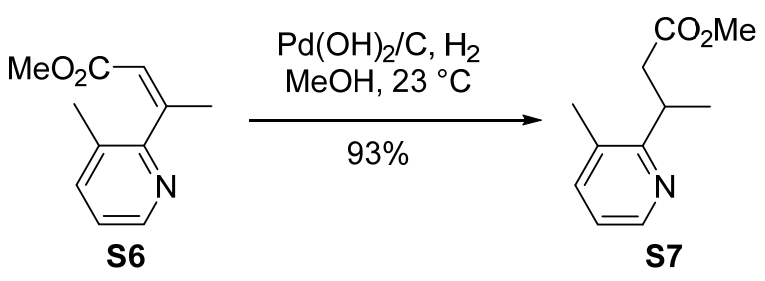

Saturated ester S7. $\mathrm{Pd}(\mathrm{OH})_{2} / \mathrm{C}(20 \mathrm{wt} \%$ loading, $57.4 \mathrm{mg}$ ) was added to a solution of alkene S6 (287 $\mathrm{mg}, 1.50 \mathrm{mmol})$ in $\mathrm{MeOH}(7.50 \mathrm{~mL})$ at $23{ }^{\circ} \mathrm{C}$. The reaction mixture was stirred at $23{ }^{\circ} \mathrm{C}$ for $3 \mathrm{~h}$ under $\mathrm{H}_{2}$ atmosphere (1 atm). The reaction mixture was filtered through a pad of Celite with EtOAc, and the filtrate was concentrated. The residue was purified by flash column chromatography on silica gel ( 5 $\mathrm{g}$, hexane/EtOAc $=4 / 1)$ to afford saturated ester $\mathbf{S 7}(270 \mathrm{mg}, 1.40 \mathrm{mmol})$ in $93 \%$ yield: colorless oil. IR (film): 2958, 1736, 1578, 1441, 1364, 1284, 1170, 1095, 1002, $791 \mathrm{~cm}^{-1} .{ }^{1} \mathrm{H}$ NMR (500 MHz, $\left.\mathrm{CDCl}_{3}\right): \delta 8.37(1 \mathrm{H}, \mathrm{d}, J=4.6 \mathrm{~Hz}, \mathrm{H} 6$ ') $7.40(1 \mathrm{H}, \mathrm{d}, J=7.5 \mathrm{~Hz}, \mathrm{H} 4$ ') $7.00(1 \mathrm{H}, \mathrm{dd}, J=7.5,4.6 \mathrm{~Hz}$, H5'), 3.61 (1H, m, H7'), 3.59 (3H, s, $\mathrm{CO}_{2} \mathrm{CH}_{3}$ ), 3.00 (1H, dd, $J=16.1,8.1 \mathrm{~Hz}, \mathrm{H} 8$ 'a), 2.63 (1H, dd, $J$ = 16.1, $\left.6.3 \mathrm{~Hz}, \mathrm{H} 8^{\prime} \mathrm{b}\right), 2.38$ (3H, s, H12'), 1.24 (3H, d, $\left.J=6.9 \mathrm{~Hz}, \mathrm{H} 9{ }^{\prime}\right) .{ }^{13} \mathrm{C}\left\{{ }^{1} \mathrm{H}\right\} \mathrm{NMR}(100 \mathrm{MHz}$, $\left.\mathrm{CDCl}_{3}\right): \delta 173.6,162.5,146.7,137.8,130.4,121.2,51.4,39.9,33.1,20.2,18.6$. HRMS (ESI-TOF) $[\mathrm{M}+\mathrm{Na}]^{+} \mathrm{m} / \mathrm{z}$ : Calcd for $\mathrm{C}_{11} \mathrm{H}_{15} \mathrm{NO}_{2} \mathrm{Na} 216.0995$; Found 216.1000 .
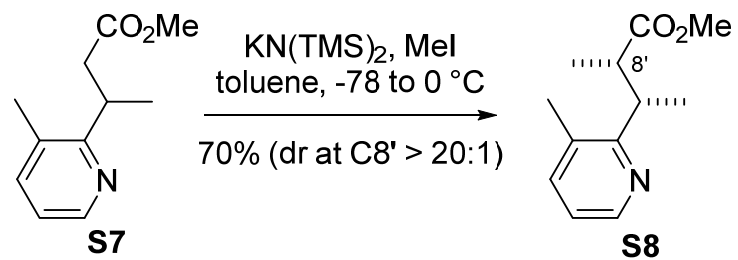

Methyl ester S8. KN(TMS) 2 (0.5 M toluene solution, $6.10 \mathrm{~mL}, 3.05 \mathrm{mmol})$ was added dropwise to a solution of saturated ester $\mathbf{S} 7(559 \mathrm{mg}, 2.90 \mathrm{mmol})$ in toluene $(10.0 \mathrm{~mL})$ at $-78^{\circ} \mathrm{C}$. After the reaction mixture was stirred at $-78^{\circ} \mathrm{C}$ for $2 \mathrm{~h}$, MeI $(380 \mu \mathrm{L}, 6.10 \mathrm{mmol})$ was added to the mixture. After the reaction mixture was stirred at $-78{ }^{\circ} \mathrm{C}$ for $19 \mathrm{~h}$ and at $0{ }^{\circ} \mathrm{C}$ for $10 \mathrm{~min}, \mathrm{pH} 7$ phosphate buffer $(8 \mathrm{~mL})$ and saturated aqueous $\mathrm{Na}_{2} \mathrm{~S}_{2} \mathrm{O}_{3}(2 \mathrm{~mL})$ were successively added to the mixture. The resultant mixture was extracted with EtOAc $\left(10 \mathrm{~mL}\right.$ x3). The combined organic layers were dried over $\mathrm{Na}_{2} \mathrm{SO}_{4}$, filtered, and concentrated. The residue was purified by automated flash column chromatography using the prepacked silica gel (16 g, hexane/EtOAc $=85 / 15$ to 64/36) to afford methyl ester S8 (290 mg, 1.40 mmol, dr at C8' > 20:1) and impure S8 (191 mg). The impure S8 was purified by automated flash column chromatography using the prepacked silica gel (16 g, hexane/EtOAc $=85 / 15$ to $64 / 36)$ to afford methyl ester S8 (133 mg, $643 \mu \mathrm{mol}$, dr at C8' > 20:1). Methyl ester S8 (423 mg, 2.04 mmol, dr 
at C8'> 20:1) was obtained in 70\% yield: colorless oil. IR (film): 2974, 2952, 1735, 1574, 1452, 1434, 1364, 1255, 1196, $1165 \mathrm{~cm}^{-1} .{ }^{1} \mathrm{H}$ NMR (400 MHz, $\left.\mathrm{CDCl}_{3}\right): \delta 8.35(1 \mathrm{H}, \mathrm{d}, J=5.0 \mathrm{~Hz}, \mathrm{H} 6$ '), $7.38(1 \mathrm{H}$, d, $J=7.8 \mathrm{~Hz}, \mathrm{H} 4$ '), 6.98 (1H, dd, $J=7.8,5.0 \mathrm{~Hz}, \mathrm{H} 5$ '), 3.45 (3H, s, $\mathrm{CO}_{2} \mathrm{CH}_{3}$ ), 3.34 (1H, m, H7'), 3.09 (1H, m, H8'), 2.36 (3H, s, H12'), 1.28 (1H, d, $J=7.3 \mathrm{~Hz}, \mathrm{H} 10 '), 1.22\left(3 \mathrm{H}, \mathrm{d}, J=7.8 \mathrm{~Hz}, \mathrm{H} 9\right.$ '). ${ }^{13} \mathrm{C}\left\{{ }^{1} \mathrm{H}\right\}$ NMR (125 MHz, $\left.\mathrm{CDCl}_{3}\right): \delta 177.0,163.0,146.5,137.7,130.4,121.0,51.3,39.2,18.7,17.1,15.3$. HRMS (ESI-TOF) $[\mathrm{M}+\mathrm{Na}]^{+} \mathrm{m} / \mathrm{z}$ : Calcd for $\mathrm{C}_{12} \mathrm{H}_{17} \mathrm{NO}_{2} \mathrm{Na} 230.1152$; Found 230.1151.
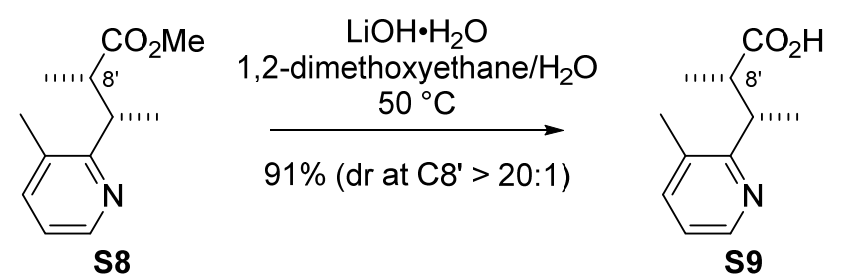

Carboxylic acid S9. $\mathrm{LiOH} \cdot \mathrm{H}_{2} \mathrm{O}(173 \mathrm{mg}, 4.12 \mathrm{mmol})$ was added to a solution of methyl ester $\mathbf{S 8}$ (418 mg, $2.02 \mathrm{mmol}$, dr at C8'> 20:1) in 1,2-dimethoxyethane (10.0 mL) and $\mathrm{H}_{2} \mathrm{O}(2.00 \mathrm{~mL})$ at $25^{\circ} \mathrm{C}$. After the reaction mixture was stirred at $50{ }^{\circ} \mathrm{C}$ for $24 \mathrm{~h}, 1 \mathrm{M}$ aqueous $\mathrm{HCl}$ was added to the mixture at $25{ }^{\circ} \mathrm{C}$ to reach $\mathrm{pH}$ 7. After the resultant mixture was concentrated, the residue was passed through a pad of silica gel $\left(5 \mathrm{~g}, \mathrm{CHCl}_{3} / \mathrm{MeOH}=1 / 0\right.$ to $\left.10 / 1\right)$ to afford the crude $\mathbf{S 9}$. The crude $\mathbf{S 9}$ was purified by automated flash column chromatography using the prepacked silica gel $\left(16 \mathrm{~g}, \mathrm{CHCl}_{3} / \mathrm{MeOH}=\right.$ $100 / 0$ to $95 / 5 ; 16 \mathrm{~g}, \mathrm{CHCl}_{3} / \mathrm{MeOH}=100 / 0$ to $95 / 5$ ) to afford carboxylic acid $\mathbf{S 9}$ (354 mg, $1.83 \mathrm{mmol}$, dr at C8' > 20:1) in 91\% yield: colorless oil. IR (film): 3730, 2969, 2926, 2875, 1700, 1653, 1565, 1454, 1412, $1023 \mathrm{~cm}^{-1} .{ }^{1} \mathrm{H}$ NMR (400 MHz, $\left.\mathrm{CDCl}_{3}\right): \delta 8.34(1 \mathrm{H}, \mathrm{dd}, J=5.0,0.9 \mathrm{~Hz}, \mathrm{H6}$ '), $7.65(1 \mathrm{H}$, dd, $J=7.8,0.9 \mathrm{~Hz}, \mathrm{H} 4$ ') 7.25 (1H, dd, $J=7.8,5.0 \mathrm{~Hz}, \mathrm{H} 5$ '), 3.32 (1H, td, $J=7.3,6.0 \mathrm{~Hz}, \mathrm{H} 7$ '), 3.09 (1H, td, $J=7.3,6.0$ Hz, H8'), 2.39 (3H, s, H12'), 1.39 (3H, d, $J=7.3 \mathrm{~Hz}, \mathrm{H} 9$ '), 1.13 (3H, d, $J=7.3$ $\mathrm{Hz}, \mathrm{H} 10$ '). ${ }^{13} \mathrm{C}\left\{{ }^{1} \mathrm{H}\right\}$ NMR (125 MHz, $\left.\mathrm{CDCl}_{3}\right): \delta 177.2,160.3,143.4,140.7,132.8,122.5,47.6,38.4$, 19.2, 18.7, 16.8. HRMS (ESI-TOF) $[\mathrm{M}-\mathrm{H}]^{-} \mathrm{m} / \mathrm{z}$ : Calcd for $\mathrm{C}_{11} \mathrm{H}_{14} \mathrm{NO}_{2}$ 192.1030; Found 192.1029.
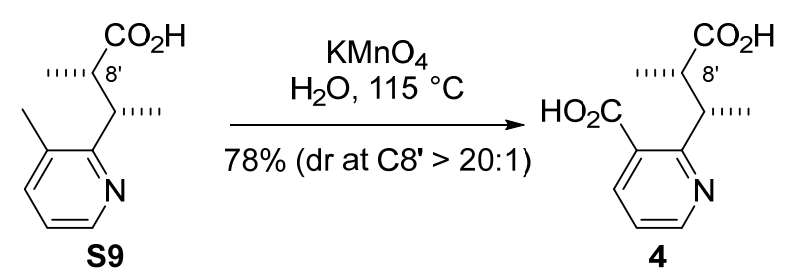

Racemic evoninic acid 4. $\mathrm{KMnO}_{4}(651 \mathrm{mg}, 4.12 \mathrm{mmol})$ was added to a solution of carboxylic acid S9 (329 mg, $1.70 \mathrm{mmol}$, dr at C8'> 20:1) in $\mathrm{H}_{2} \mathrm{O}(8.50 \mathrm{~mL})$ at $25^{\circ} \mathrm{C}$. After the reaction mixture was stirred at $115{ }^{\circ} \mathrm{C}$ for $10 \mathrm{~h}, \mathrm{KMnO}_{4}(641 \mathrm{mg}, 4.06 \mathrm{mmol})$ was added to the mixture at $25^{\circ} \mathrm{C}$. After the reaction mixture was stirred at $115{ }^{\circ} \mathrm{C}$ for $5 \mathrm{~h}, \mathrm{KMnO}_{4}(132 \mathrm{mg}, 835 \mu \mathrm{mol})$ was added to the mixture at $25^{\circ} \mathrm{C}$. After the reaction mixture was stirred at $115^{\circ} \mathrm{C}$ for $2 \mathrm{~h}, 1 \mathrm{M}$ aqueous $\mathrm{HCl}$ was added to the 
mixture at $25^{\circ} \mathrm{C}$ to reach $\mathrm{pH}$ 6. After $\mathrm{MeOH}(13 \mathrm{~mL})$ was added to the mixture, the resultant mixture was passed through a pad of Celite with $\mathrm{MeOH}$. After the resultant solution was concentrated, the residue was passed through a pad of silica gel $\left(8 \mathrm{~g}, \mathrm{CHCl}_{3} / \mathrm{MeOH}=5 / 1\right.$ to $\left.1 / 1\right)$ to afford a mixture of 4 and inorganic salt. Inorganic salt was removed by cotton filtration with $\mathrm{CHCl}_{3} / \mathrm{MeOH}(5 / 1)$ to afford racemic evoninic acid 4 (295 mg, $1.32 \mathrm{mmol}$, dr at C8'> 20:1) in 78\% yield: white amorphous. IR (film): 3749, 3674, 3650, 2975, 2930, 1702, 1562, 1541, 1456, $1390 \mathrm{~cm}^{-1} .{ }^{1} \mathrm{H}$ NMR (400 MHz, $\left.\mathrm{CD}_{3} \mathrm{OD}\right): \delta 8.52\left(1 \mathrm{H}, \mathrm{dd}, J=5.0,1.8 \mathrm{~Hz}, \mathrm{H6}{ }^{\prime}\right), 8.08\left(1 \mathrm{H}, \mathrm{dd}, J=7.8,1.8 \mathrm{~Hz}, \mathrm{H} 4^{\prime}\right), 7.32(1 \mathrm{H}, \mathrm{dd}, J=$ 7.8, 5.2 Hz, H5'), 3.99 (1H, dq, $J=15.1,7.3$ Hz, H7'), 3.02 (1H, dq, J = 15.1, 7.3 Hz, H8'), 1.32 (3H, $\mathrm{d}, J=7.3 \mathrm{~Hz}, \mathrm{H} 9 '), 1.24\left(3 \mathrm{H}, \mathrm{d}, J=7.3 \mathrm{~Hz}, \mathrm{H} 10^{\prime}\right) .{ }^{13} \mathrm{C}\left\{{ }^{1} \mathrm{H}\right\} \mathrm{NMR}\left(125 \mathrm{MHz}, \mathrm{CD}_{3} \mathrm{OD}\right): \delta 180.7,172$ (deduced from HMBC), 163.8, 149.7, 139.4, 133 (deduced from HMBC), 122.8, 47.1, 41.0, 18.8, 16.4. HRMS (ESI-TOF) $[\mathrm{M}-\mathrm{H}]^{-} \mathrm{m} / \mathrm{z}$ : Calcd for $\mathrm{C}_{11} \mathrm{H}_{12} \mathrm{NO}_{4}$ 222.0772; Found 222.0768.
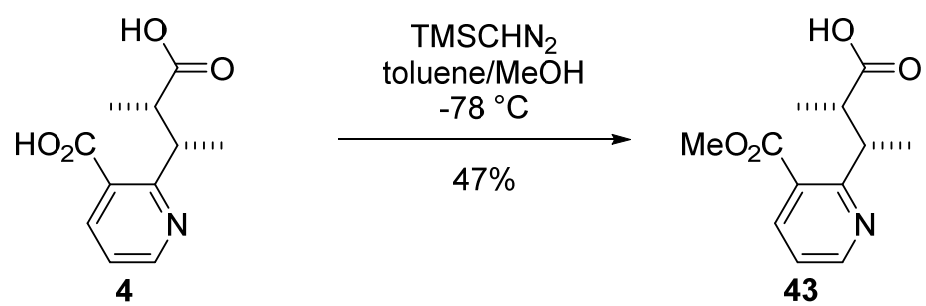

Evoninic acid methyl ester 43. $\mathrm{TMSCHN}_{2}(0.6 \mathrm{M}$ hexane solution, $160 \mu \mathrm{L}, 96.0 \mu \mathrm{mol})$ was added to a solution of racemic evoninic acid $4(19.6 \mathrm{mg}, 87.9 \mu \mathrm{mol}$, dr at C8'> 20:1) in toluene $(440 \mu \mathrm{L})$ and $\mathrm{MeOH}(440 \mu \mathrm{L})$ at $-78{ }^{\circ} \mathrm{C}$. After the reaction mixture was stirred at $-78{ }^{\circ} \mathrm{C}$ for $1 \mathrm{~h}, \mathrm{AcOH}(\mathrm{ca} .10 \mu \mathrm{L})$ was added to the mixture. The resultant mixture was warmed to $25^{\circ} \mathrm{C}$ and concentrated. The residue was passed through a pad of silica gel $\left(0.5 \mathrm{~g}, \mathrm{CHCl}_{3} / \mathrm{MeOH}=100 / 1\right.$ to $\left.1 / 1\right)$ and concentrated. The residue was purified by PTLC (20 cm x $\left.10 \mathrm{~cm}, \mathrm{CHCl}_{3} / \mathrm{MeOH}=10 / 1\right)$ to afford 43 (9.70 $\mathrm{mg}, 40.9$ $\mu \mathrm{mol}$, dr at C8' = 13:1) in 47\% yield: white amorphous. IR (film): 2972, 1724, 1577, 1439, 1270, 1201, 1130, 1082, 789, $740 \mathrm{~cm}^{-1}$. ${ }^{1} \mathrm{H}$ NMR (500 MHz, $\left.\mathrm{CDCl}_{3}\right): \delta 8.64(1 \mathrm{H}, \mathrm{dd}, J=5.2,1.8 \mathrm{~Hz}, \mathrm{H} 6$ '), 8.33 (1H, dd, $J=8.1,1.8 \mathrm{~Hz}, \mathrm{H} 4^{\prime}$ ), 7.42 (1H, dd, $\left.J=8.1,5.2 \mathrm{~Hz}, \mathrm{H} 5^{\prime}\right), 4.12$ (1H, dq, $J=7.5,7.5$ Hz, H7'), $3.97\left(3 \mathrm{H}, \mathrm{s}, \mathrm{CO}_{2} \mathrm{CH}_{3}\right), 3.10$ (1H, dq, $J=7.5,7.5 \mathrm{~Hz}, \mathrm{H} 8$ '), 1.45 (3H, d, $J=7.5 \mathrm{~Hz}, \mathrm{H} 9$ '), 1.12 (3H, d, $J=7.5 \mathrm{~Hz}, \mathrm{H} 10 ') .{ }^{13} \mathrm{C}\left\{{ }^{1} \mathrm{H}\right\} \mathrm{NMR}\left(125 \mathrm{MHz}, \mathrm{CDCl}_{3}\right): \delta 177.6,166.1,163.8,149.5,140.2,126.9,121.9$, 52.8, 46.6, 39.0, 19.6, 16.1. HRMS (ESI-TOF) $[\mathrm{M}-\mathrm{H}+2 \mathrm{Na}]^{+} \mathrm{m} / \mathrm{z}$ : Calcd for $\mathrm{C}_{12} \mathrm{H}_{14} \mathrm{NO}_{4} \mathrm{Na}_{2} 282.0713$; Found 282.0719. 


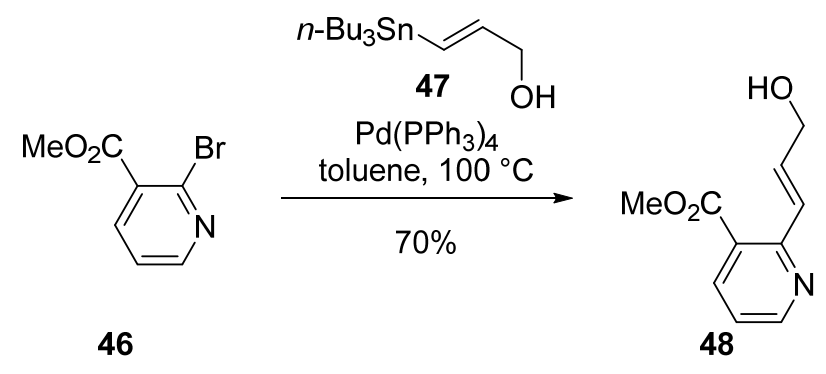

Alcohol 48. $\mathrm{Pd}\left(\mathrm{PPh}_{3}\right)_{4}(284 \mathrm{mg}, 246 \mu \mathrm{mol})$ was added to a solution of bromide 46 (1.06 g, $\left.4.91 \mathrm{mmol}\right)$ and stannane $47(2.05 \mathrm{~g}, 5.91 \mathrm{mmol})$ in toluene $(14.0 \mathrm{~mL})$ at $25^{\circ} \mathrm{C}$. The mixture was degassed by freeze-thaw procedure (x3). The reaction mixture was stirred at $100{ }^{\circ} \mathrm{C}$ for $26 \mathrm{~h}$. After being cooled to $25^{\circ} \mathrm{C}$, the reaction mixture was directly passed through a column (consecutively packed with silica gel $15 \mathrm{~g}$ and $10 \mathrm{wt} \% \mathrm{KF}$ contained silica gel $7.5 \mathrm{~g}$, hexane/EtOAc $=10 / 1$ to $0 / 1$ ) and concentrated. The residue was purified by automated flash column chromatography using the prepacked silica gel (40 g x2, $\mathrm{CHCl}_{3} / \mathrm{MeOH}=100 / 0$ to $\left.94 / 6\right)$ to afford alcohol 48 (661 mg, $3.42 \mathrm{mmol}$ ) in $70 \%$ yield: light brown solid. m.p. 40-41 ${ }^{\circ} \mathrm{C}$. IR (film) 3347, 1722, 1650, 1580, 1562, 1429, 1289, 1264, 1137, 1084 $\mathrm{cm}^{-1} .{ }^{1} \mathrm{H}$ NMR $\left(400 \mathrm{MHz}, \mathrm{CDCl}_{3}\right) \delta 8.70(1 \mathrm{H}, \mathrm{dd}, J=4.6,1.8 \mathrm{~Hz}, \mathrm{H6}$ ') $8.21(1 \mathrm{H}, \mathrm{dd}, J=8.2,1.8 \mathrm{~Hz}$, H4'), 7.56 (1H, ddd, $J=15.6,1.8,1.8 \mathrm{~Hz}, \mathrm{H} 7$ ') 7.27 (1H, dd, $J=8.2,4.6 \mathrm{~Hz}, \mathrm{H} 5$ '), 7.21 (1H, ddd, $J$ $\left.=15.6,5.0,5.0 \mathrm{~Hz}, \mathrm{H} 8^{\prime}\right), 4.45\left(2 \mathrm{H}, \mathrm{d}, J=5.0,1.8 \mathrm{~Hz}, \mathrm{H} 11^{\prime}\right), 3.94\left(3 \mathrm{H}, \mathrm{s}, \mathrm{CO}_{2} \mathrm{CH}_{3}\right) .{ }^{13} \mathrm{C}\left\{{ }^{1} \mathrm{H}\right\} \mathrm{NMR}$ $\left(100 \mathrm{MHz} \mathrm{CDCl}_{3}\right) \delta 166.6,154.7,151.6,138.4,137.7,125.9,123.7,121.3,62.6,52.2$. HRMS (ESITOF) $[\mathrm{M}+\mathrm{Na}]^{+} \mathrm{m} / \mathrm{z}$ : Calcd for $\mathrm{C}_{10} \mathrm{H}_{11} \mathrm{NO}_{3} \mathrm{Na} 216.0631$; Found 216.0638.
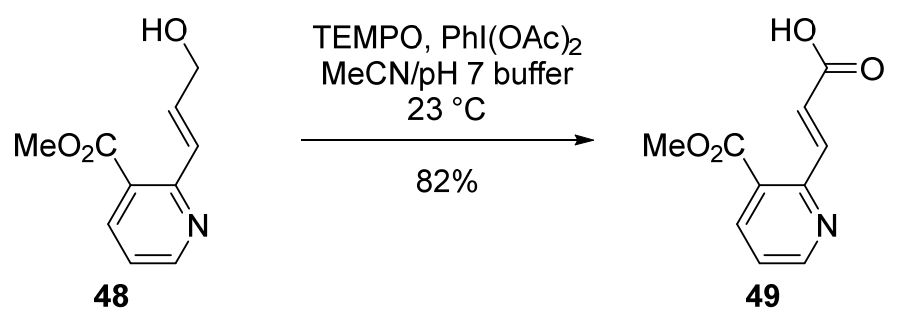

Carboxylic acid 49. 2,2,6,6-Tetramethylpiperidine 1-oxyl (TEMPO, $108 \mathrm{mg}, 694 \mu \mathrm{mol}$ ) and $\mathrm{PhI}(\mathrm{OAc})_{2}(5.60 \mathrm{~g}, 17.4 \mathrm{mmol})$ were successively added to a solution of alcohol 48 (671 $\mathrm{mg}, 3.47$ $\mathrm{mmol})$ in $\mathrm{MeCN}(8.70 \mathrm{~mL})$ and $\mathrm{pH} 7$ phosphate buffer $(8.70 \mathrm{~mL})$ at $23{ }^{\circ} \mathrm{C}$. After the reaction mixture was stirred at $23{ }^{\circ} \mathrm{C}$ for $24 \mathrm{~h}$, saturated aqueous $\mathrm{Na}_{2} \mathrm{~S}_{2} \mathrm{O}_{3}(17 \mathrm{~mL})$ was added to the mixture at $0{ }^{\circ} \mathrm{C}$. The resultant mixture was extracted with EtOAc $(20 \mathrm{~mL}$ x8). The combined organic layers were dried over $\mathrm{Na}_{2} \mathrm{SO}_{4}$, filtered, and concentrated. The residue was purified by flash column chromatography on silica gel ( $50 \mathrm{~g}, \mathrm{CHCl}_{3} / \mathrm{MeOH}=100 / 1$ to 5/1) to afford carboxylic acid 49 (587 $\left.\mathrm{mg}, 2.83 \mathrm{mmol}\right)$ in $82 \%$ yield. white solid. m.p. 190-191 ${ }^{\circ} \mathrm{C}$. IR (film) 2941, 2365, 2328, 1716, 1565, 1256, 1194, 1135 , 1056, $1037 \mathrm{~cm}^{-1} .{ }^{1} \mathrm{H}$ NMR (400 MHz, CD $\left.3 \mathrm{OD}\right) \delta 8.74(1 \mathrm{H}, \mathrm{dd}, J=4.6,1.8 \mathrm{~Hz}, \mathrm{H} 6$ '), 8.47 (1H, d, $J=$ $15.6 \mathrm{~Hz}, \mathrm{H} 7$ '), 8.30 (1H, dd, $J=8.2,1.8 \mathrm{~Hz}, \mathrm{H} 4$ ') 7.49 (1H, dd, $J=8.2,4.6 \mathrm{~Hz}, \mathrm{H} 5$ ') 7.00 (1H, d, $J$ 
$\left.=15.6 \mathrm{~Hz}, \mathrm{H} 8^{\prime}\right), 3.96\left(3 \mathrm{H}, \mathrm{s}, \mathrm{CO}_{2} \mathrm{CH}_{3}\right) .{ }^{13} \mathrm{C}\left\{{ }^{1} \mathrm{H}\right\} \mathrm{NMR}\left(100 \mathrm{MHz}, \mathrm{CD}_{3} \mathrm{OD}\right) \delta 169.9,167.5,153.6$, 153.4, 141.9, 140.0, 127.7, 126.3, 125.3, 53.3. HRMS (ESI-TOF) $[\mathrm{M}-\mathrm{H}+2 \mathrm{Na}]^{+} \mathrm{m} / \mathrm{z}$ : Calcd for $\mathrm{C}_{10} \mathrm{H}_{8} \mathrm{NO}_{4} \mathrm{Na}_{2} 252.0243$; Found 252.0254.
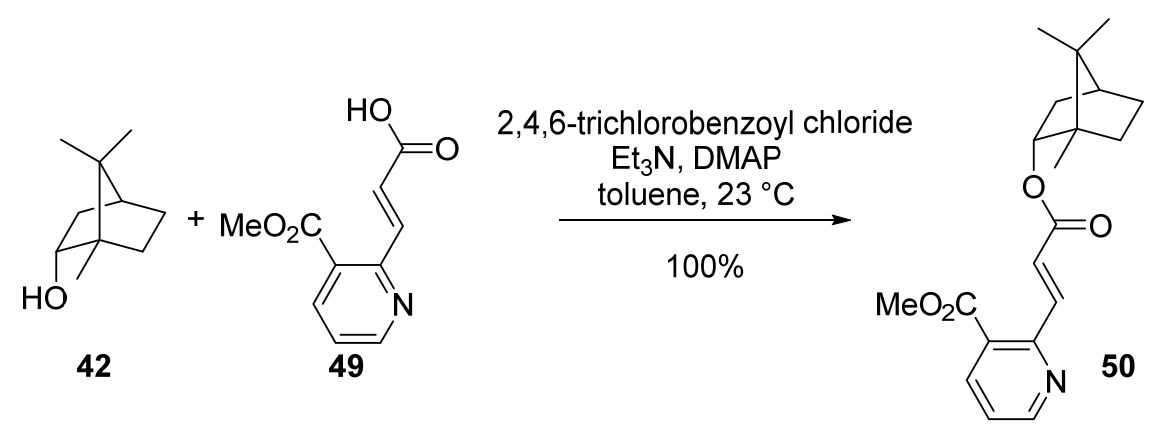

Ester 50. 2,4,6-Trichlorobenzoyl chloride $(7.3 \mu \mathrm{L}, 47 \mu \mathrm{mol})$ was added to a solution of borneol 42 (2.45 mg, $15.9 \mu \mathrm{mol})$, carboxylic acid 49 (6.4 mg, $31 \mu \mathrm{mol}), \mathrm{Et}_{3} \mathrm{~N}(8.7 \mu \mathrm{L}, 63 \mu \mathrm{mol})$, and DMAP (5.7 $\mathrm{mg}, 47 \mu \mathrm{mol})$ in toluene $(390 \mu \mathrm{L})$ at $23{ }^{\circ} \mathrm{C}$. After the reaction mixture was stirred at $23{ }^{\circ} \mathrm{C}$ for $1 \mathrm{~h}$, saturated aqueous $\mathrm{NH}_{4} \mathrm{Cl}(0.4 \mathrm{~mL})$ was added to the mixture. The resultant mixture was extracted with EtOAc $(1 \mathrm{~mL} \times 3)$. The combined organic layers were dried over $\mathrm{Na}_{2} \mathrm{SO}_{4}$, filtered, and concentrated. The residue was purified by flash column chromatography on silica gel (0.5 g, hexane/EtOAc $=10 / 1$ to $3 / 1)$ to afford ester $\mathbf{5 0}(5.46 \mathrm{mg}, 15.9 \mu \mathrm{mol})$ in $100 \%$ yield: colorless oil. $[\alpha]_{\mathrm{D}}^{22}-30.3\left(c\right.$ 0.585, $\left.\mathrm{CHCl}_{3}\right)$. IR (film) 2954, 2880, 1721, 1434, 1300, 1275, 1190, 1075, $1029 \mathrm{~cm}^{-1}$. ${ }^{1} \mathrm{H}$ NMR (500 MHz, $\left.\mathrm{CDCl}_{3}\right) \delta 8.75(1 \mathrm{H}, \mathrm{dd}, J=4.6,1.7 \mathrm{~Hz}, \mathrm{H6}$ '), 8.48 (1H, d, $J=15.5 \mathrm{~Hz}, \mathrm{H} 7$ '), 8.23 $(1 \mathrm{H}, \mathrm{dd}, J=8.0,1.7 \mathrm{~Hz}, \mathrm{H} 4$ ') 7.36 (1H, dd, $J=8.0,4.6 \mathrm{~Hz}, \mathrm{H} 5$ '), 7.14 (1H, d, $J=15.5 \mathrm{~Hz}, \mathrm{H} 8$ '), 5.02 $(1 \mathrm{H}, \mathrm{ddd}, J=10.3,3.5,2.3 \mathrm{~Hz}), 3.98\left(3 \mathrm{H}, \mathrm{s}, \mathrm{CO}_{2} \mathrm{CH}_{3}\right), 2.43(1 \mathrm{H}, \mathrm{m}), 2.07(1 \mathrm{H}, \mathrm{ddd}, J=13.2,9.2,4.0$ $\mathrm{Hz}), 1.77(1 \mathrm{H}, \mathrm{m}), 1.71(1 \mathrm{H}, \mathrm{dd}, J=4.6,4.6 \mathrm{~Hz}), 1.35(1 \mathrm{H}, \mathrm{m}), 1.27(1 \mathrm{H}, \mathrm{m}), 1.07(1 \mathrm{H}, \mathrm{dd}, J=13.8$, $3.5 \mathrm{~Hz}), 0.94(3 \mathrm{H}, \mathrm{s}), 0.90(3 \mathrm{H}, \mathrm{s}), 0.88(3 \mathrm{H}, \mathrm{s}) .{ }^{13} \mathrm{C}\left\{{ }^{1} \mathrm{H}\right\} \mathrm{NMR}\left(125 \mathrm{MHz}, \mathrm{CDCl}_{3}\right) \delta$ 166.9, 166.4, 152.7, 152.2, 140.2, 138.7, 126.2, 125.9, 123.5, 80.2, 52.8, 48.9, 47.8, 44.9, 36.9, 28.0, 27.1, 19.7, 18.9, 13.5. HRMS (ESI-TOF) $[\mathrm{M}+\mathrm{Na}]^{+} \mathrm{m} / \mathrm{z}$ : Calcd for $\mathrm{C}_{20} \mathrm{H}_{25} \mathrm{NO}_{4} \mathrm{Na}$ 366.1676; Found 366.1685. 


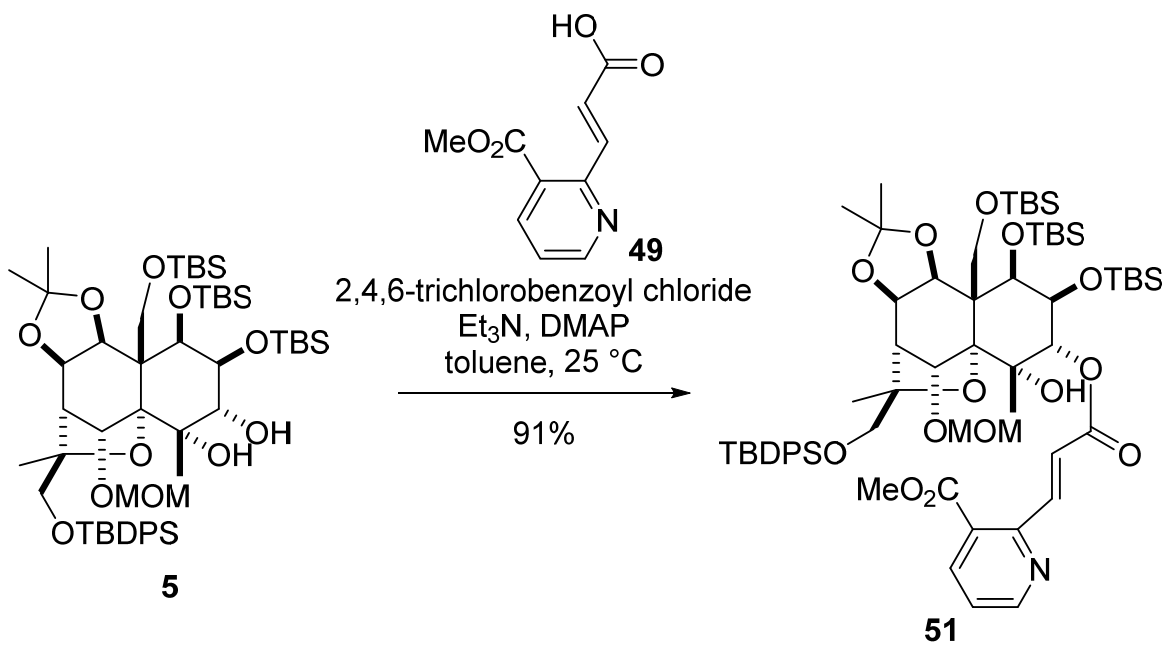

Ester 51. 2,4,6-Trichlorobenzoyl chloride (138 $\mu \mathrm{L}, 882 \mu \mathrm{mol})$ was dissolved in toluene $(600 \mu \mathrm{L})$. The above solution of 2,4,6-trichlorobenzoyl chloride $(1.20 \mathrm{M}, 369 \mu \mathrm{L}, 443 \mu \mathrm{mol})$ was added to a solution of protected euonyminol $5(65.2 \mathrm{mg}, 63.2 \mu \mathrm{mol})$, carboxylic acid 49 (91.8 mg, $443 \mu \mathrm{mol})$, $\mathrm{Et}_{3} \mathrm{~N}(106 \mu \mathrm{L}, 762 \mu \mathrm{mol})$, and DMAP $(54.2 \mathrm{mg}, 444 \mu \mathrm{mol})$ in toluene $(1.30 \mathrm{~mL})$ at $25^{\circ} \mathrm{C}$ via syringe pump over $6 \mathrm{~h}$. After the addition was completed, the reaction mixture was stirred at $25^{\circ} \mathrm{C}$ for $15 \mathrm{~h}$, and then saturated aqueous $\mathrm{NH}_{4} \mathrm{Cl}(2 \mathrm{~mL})$ was added to the mixture at $0{ }^{\circ} \mathrm{C}$. The resultant mixture was extracted with EtOAc $(2 \mathrm{~mL} \times 3)$. The combined organic layers were directly passed through a pad of silica gel $(0.5 \mathrm{~g}$, EtOAc) and concentrated. The residue was purified by automated flash column chromatography using the prepacked silica gel ( $7 \mathrm{~g}$, hexane/EtOAc $=97 / 3$ to 76/24) to afford ester 51 (70.1 mg, $57.4 \mu \mathrm{mol})$ in 91\% yield: white amorphous. $[\alpha]_{\mathrm{D}}{ }^{25}+7.03$ (c 1.12, $\mathrm{CHCl}_{3}$ ). IR (film): 2943 , 2860, 1723, 1262, 1082, 881, 836, $778 \mathrm{~cm}^{-1} .{ }^{1} \mathrm{H}$ NMR (400 MHz, $\left.\mathrm{CDCl}_{3}\right): \delta 8.71(1 \mathrm{H}, \mathrm{dd}, J=4.6,1.8$ Hz, H6'), 8.50 (1H, d, $J=15.6 \mathrm{~Hz}, \mathrm{H}^{\prime}$ '), 8.22 (1H, dd, $J=7.8,1.8 \mathrm{~Hz}, \mathrm{H} 4$ '), 7.58-7.53 (4H, m, aromatic), $7.35\left(1 \mathrm{H}, \mathrm{dd}, J=7.8,4.6 \mathrm{~Hz}, \mathrm{H} 5{ }^{\prime}\right), 7.31-7.28$ (2H, m, aromatic), 7.24-7.17 (4H, m, aromatic), $7.06(1 \mathrm{H}, \mathrm{d}, J=15.6 \mathrm{~Hz}, \mathrm{H} 8$ ') $5.60(1 \mathrm{H}, \mathrm{s}, \mathrm{H} 6), 5.07(1 \mathrm{H}, \mathrm{d}, J=1.8 \mathrm{~Hz}, \mathrm{H} 3), 4.78(1 \mathrm{H}, \mathrm{d}$, $J=12.4 \mathrm{~Hz}, \mathrm{H} 14 \mathrm{a}), 4.57-4.51\left(3 \mathrm{H}, \mathrm{m}, \mathrm{H} 8, \mathrm{OCH}_{2} \mathrm{OCH}_{3}\right), 4.25(1 \mathrm{H}, \mathrm{d}, J=4.1 \mathrm{~Hz}, \mathrm{H} 1), 4.16(1 \mathrm{H}, \mathrm{d}, J$ $=12.4 \mathrm{~Hz}, \mathrm{H} 14 \mathrm{~b}), 4.03(1 \mathrm{H}, \mathrm{d}, J=7.8 \mathrm{~Hz}, \mathrm{H} 9), 3.99(1 \mathrm{H}, \mathrm{d}, J=10.0 \mathrm{~Hz}, \mathrm{H} 13 \mathrm{a}), 3.91\left(3 \mathrm{H}, \mathrm{s}, \mathrm{CO}_{2} \mathrm{CH}_{3}\right)$, $3.87(1 \mathrm{H}, \mathrm{dd}, J=4.1,1.8 \mathrm{~Hz}, \mathrm{H} 2), 3.79(1 \mathrm{H}, \mathrm{d}, J=10.0 \mathrm{~Hz}, \mathrm{H} 13 \mathrm{~b}), 3.53(1 \mathrm{H}, \mathrm{s}, \mathrm{OH}), 3.06$ (3H, s, $\left.\mathrm{OCH}_{2} \mathrm{OCH}_{3}\right), 2.65(1 \mathrm{H}, \mathrm{d}, J=2.3 \mathrm{~Hz}, \mathrm{H} 7), 1.69(3 \mathrm{H}, \mathrm{s}, \mathrm{H} 15), 1.55$ (3H, s, $\mathrm{CH}_{3}$ of acetonide), 1.37 (3H, s, H12), 1.31 (3H, s, CH3 of acetonide), 0.97 (9H, s, $t$-Bu of TBS), 0.94 (9H, s, $t$-Bu of TBS), 0.93 (9H, s, $t$-Bu of TBS), 0.89 (9H, s, $t$-Bu of TBDPS), $0.213\left(3 \mathrm{H}, \mathrm{s}, \mathrm{CH}_{3}\right.$ of TBS), 0.207 (3H, s, $\mathrm{CH}_{3}$ of TBS), 0.13 (3H, s, CH3 of TBS), 0.11 (3H, s, $\mathrm{CH}_{3}$ of TBS), 0.07 (3H, s, CH3 of TBS), 0.03 (3H, s, $\mathrm{CH}_{3}$ of TBS). ${ }^{13} \mathrm{C}\left\{{ }^{1} \mathrm{H}\right\}$ NMR (100 MHz, $\left.\mathrm{CDCl}_{3}\right): \delta 165.8,165.7,153.0,152.1,140.8,138.5,135.75$ (2C), 135.69 (2C), 133.5, 133.2, 129.44, 129.37, 127.44 (2C), 127.35 (2C), 126.0, 125.7, 123.3, 108.3, 97.2, $93.2,83.5,82.2,80.2,78.0,75.9,75.5,74.3,70.5,68.8,60.1,56.2,56.0,52.7,46.6,27.2$ (3C), 27.0, 
26.8 (3C), 26.5 (3C), 26.4 (3C), 23.9, 23.8, 21.3, 19.1, 18.6, 18.5, 18.0, -2.7, -3.8, -4.3, -4.4 (2C), 4.5. HRMS (ESI-TOF) $[\mathrm{M}+\mathrm{Na}]^{+} \mathrm{m} / \mathrm{z}$ : Calcd for $\mathrm{C}_{64} \mathrm{H}_{101} \mathrm{NO}_{14} \mathrm{Si}_{4} \mathrm{Na} 1242.6191$; Found 1242.6217.
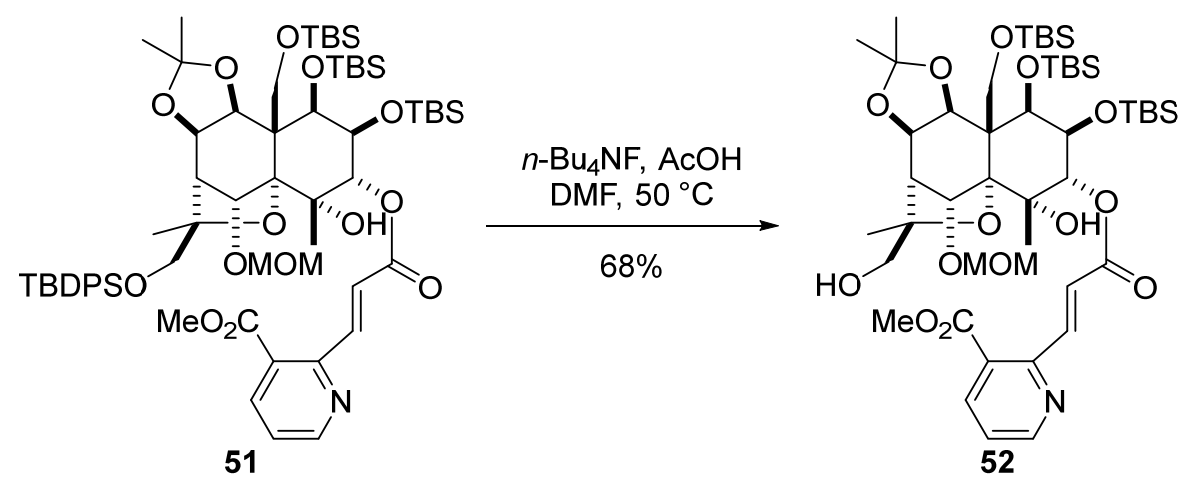

Alcohol 52. $n$-Bu4NF (1 M THF solution, $350 \mu \mathrm{L}, 350 \mu \mathrm{mol})$ was added to a solution of ester 51 (10.7 $\mathrm{mg}, 8.76 \mu \mathrm{mol})$ and $\mathrm{AcOH}(20.0 \mu \mathrm{L}, 350 \mu \mathrm{mol})$ in DMF $(880 \mu \mathrm{L})$ at $25^{\circ} \mathrm{C}$. After the reaction mixture was stirred at $50{ }^{\circ} \mathrm{C}$ for $24 \mathrm{~h}, \mathrm{pH} 7$ phosphate buffer $(2 \mathrm{~mL})$ was added to the mixture at $0{ }^{\circ} \mathrm{C}$. The resultant mixture was extracted with $\mathrm{Et}_{2} \mathrm{O}(2 \mathrm{~mL} \times 5)$. The combined organic layers were washed with $\mathrm{H}_{2} \mathrm{O}$ (2 mL x2), dried over $\mathrm{Na}_{2} \mathrm{SO}_{4}$, filtered, and concentrated. The residue was purified by PTLC (20 $\mathrm{cm} \times 10 \mathrm{~cm}$, hexane/EtOAc $=1 / 2)$ to afford alcohol $\mathbf{5 2}(5.86 \mathrm{mg}, 5.97 \mu \mathrm{mol})$ in $68 \%$ yield: white amorphous. $[\alpha]_{\mathrm{D}}{ }^{25}+26.6$ (c 0.293, $\mathrm{CHCl}_{3}$ ). IR (film): 3513, 2942, 2860, 1723, 1373, 1263, 1110, 880, 840, $778 \mathrm{~cm}^{-1} .{ }^{1} \mathrm{H}$ NMR $\left(500 \mathrm{MHz}, \mathrm{CDCl}_{3}\right): \delta 8.75(1 \mathrm{H}, \mathrm{dd}, J=4.6,1.7 \mathrm{~Hz}, \mathrm{H6}$ '), $8.59(1 \mathrm{H}, \mathrm{d}, J=$ 15.5 Hz, H7'), 8.20 (1H, dd, $J=8.0,1.7$ Hz, H4'), 7.33 (1H, dd, J = 8.0, 4.6 Hz, H5'), 7.11 (1H, d, $J$ $=15.5 \mathrm{~Hz}, \mathrm{H} 8$ '), $5.62(1 \mathrm{H}, \mathrm{s}, \mathrm{H} 6), 5.23(1 \mathrm{H}, \mathrm{d}, J=2.3 \mathrm{~Hz}, \mathrm{H} 3), 4.76\left(1 \mathrm{H}, \mathrm{d}, J=6.3 \mathrm{~Hz}, \mathrm{OCHaHbOCH}_{3}\right)$, $4.73(1 \mathrm{H}, \mathrm{d}, J=12.6 \mathrm{~Hz}, \mathrm{H14a}), 4.69\left(1 \mathrm{H}, \mathrm{d}, J=6.3 \mathrm{~Hz}, \mathrm{OCHaHbOCH}_{3}\right), 4.65(1 \mathrm{H}, \mathrm{dd}, J=7.5,2.3$ $\mathrm{Hz}, \mathrm{H} 8), 4.37(1 \mathrm{H}, \mathrm{d}, J=4.0 \mathrm{~Hz}, \mathrm{H} 1), 4.18(1 \mathrm{H}, \mathrm{d}, J=10.9 \mathrm{~Hz}, \mathrm{H} 13 \mathrm{a}), 4.15(1 \mathrm{H}, \mathrm{d}, J=12.6 \mathrm{~Hz}$, H14b), $4.03(1 \mathrm{H}, \mathrm{d}, J=7.5 \mathrm{~Hz}, \mathrm{H} 9), 3.92\left(3 \mathrm{H}, \mathrm{s}, \mathrm{CO}_{2} \mathrm{CH}_{3}\right), 3.91(1 \mathrm{H}, \mathrm{m}, \mathrm{H} 2), 3.56(1 \mathrm{H}, \mathrm{s}, \mathrm{OH}), 3.35$ $\left(3 \mathrm{H}, \mathrm{s}, \mathrm{OCH}_{2} \mathrm{OCH}_{3}\right), 3.28(1 \mathrm{H}, \mathrm{m}, \mathrm{OH}), 3.13(1 \mathrm{H}, \mathrm{m}, \mathrm{H} 13 \mathrm{~b}), 2.60(1 \mathrm{H}, \mathrm{d}, J=2.3 \mathrm{~Hz}, \mathrm{H} 7), 1.73(3 \mathrm{H}$, s, H15), 1.55 (3H, s, CH3 of acetonide), 1.41 (3H, s, H12), 1.31 (3H, s, $\mathrm{CH}_{3}$ of acetonide), 0.97 (9H, s, $t$-Bu of TBS), 0.95 (9H, s, $t$-Bu of TBS), 0.93 (9H, s, $t$-Bu of TBS), 0.22 (3H, s, CH3 of TBS), 0.21 (3H, s, $\mathrm{CH}_{3}$ of TBS), 0.14 (3H, s, CH3 of TBS), 0.11 (3H, s, $\mathrm{CH}_{3}$ of TBS), 0.069 (3H, s, $\mathrm{CH}_{3}$ of TBS), 0.065 (3H, s, $\mathrm{CH}_{3}$ of TBS). ${ }^{13} \mathrm{C}\left\{{ }^{1} \mathrm{H}\right\}$ NMR (125 MHz, $\left.\mathrm{CDCl}_{3}\right): \delta 166.0,165.7,153.0,152.3,141.6,138.5$, 126.1, 124.9, 123.5, 108.3, 98.0, 92.2, 84.0, 82.6, 78.8, 78.0, 75.9, 75.6, 74.7, 71.7, 65.5, 59.9, 56.9, 56.0, 52.7, 47.1, 27.00, 26.97 (3C), 26.43 (3C), 26.42 (3C), 25.5, 23.7, 20.8, 18.5, 18.4, 18.1, -2.5, $4.1,-4.26,-4.30,-4.6$ (2C). HRMS (ESI-TOF) $[\mathrm{M}+\mathrm{Na}]^{+} \mathrm{m} / \mathrm{z}$ : Calcd for $\mathrm{C}_{48} \mathrm{H}_{83} \mathrm{NO}_{14} \mathrm{Si}_{3} \mathrm{Na}$ 1004.5014; Found 1004.4990. 


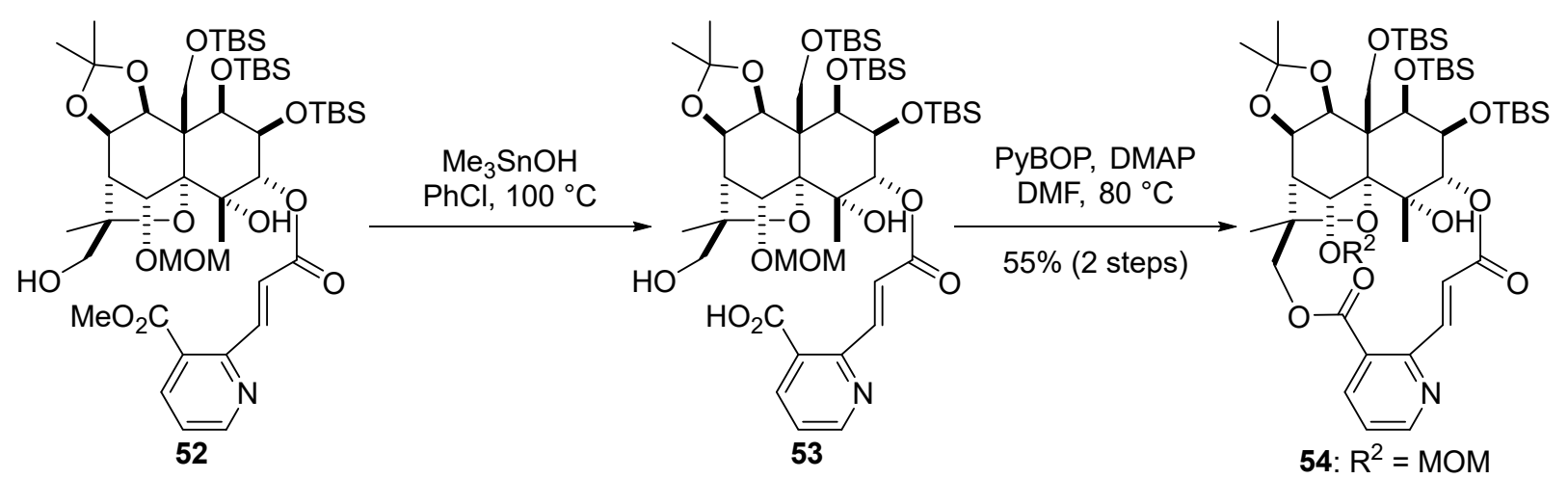

Bislactone 54. $\mathrm{Me}_{3} \mathrm{SnOH}(86.4 \mathrm{mg}, 477 \mu \mathrm{mol})$ was added to a solution of alcohol 52 (5.86 mg, 5.97 $\mu \mathrm{mol})$ in $\mathrm{PhCl}(1.20 \mathrm{~mL})$ at $23{ }^{\circ} \mathrm{C}$. The reaction mixture was stirred at $100{ }^{\circ} \mathrm{C}$ for $20 \mathrm{~h}$. After being cooled to $23{ }^{\circ} \mathrm{C}$, the reaction mixture was directly passed through a pad of silica gel $\left(4 \mathrm{~g}, \mathrm{CHCl}_{3} / \mathrm{MeOH}\right.$ $=1 / 0$ to 20/1) and concentrated to afford the crude seco acid $\mathbf{5 3}(6.45 \mathrm{mg})$, which was used in the next reaction without further purification. HRMS (ESI-TOF) $[\mathrm{M}-\mathrm{H}]^{-} \mathrm{m} / \mathrm{z}$ : Calcd for $\mathrm{C}_{47} \mathrm{H}_{80} \mathrm{NO}_{14} \mathrm{Si}_{3}$ 966.4892; Found 966.4864.

The above crude $53(6.45 \mathrm{mg})$ was azeotroped with toluene $(2 \mathrm{~mL}$ x3) and dissolved in DMF (1.20 $\mathrm{mL})$ DMAP $(4.4 \mathrm{mg}, \quad 36 \mu \mathrm{mol})$ and (benzotriazol-1-yloxy)tripyrrolidinophosphonium hexafluorophosphate (PyBOP, $9.3 \mathrm{mg}, 18 \mu \mathrm{mol}$ ) were successively added to the solution of $\mathbf{5 3}$ in DMF at $23{ }^{\circ} \mathrm{C}$. After the reaction mixture was stirred at $80{ }^{\circ} \mathrm{C}$ for $6 \mathrm{~h}$, saturated aqueous $\mathrm{NH}_{4} \mathrm{Cl}(2 \mathrm{~mL})$ was added to the mixture at $24{ }^{\circ} \mathrm{C}$. The resultant mixture was extracted with $\mathrm{Et}_{2} \mathrm{O}(2 \mathrm{~mL} \times 5)$. The combined organic layers were washed with $\mathrm{H}_{2} \mathrm{O}(2 \mathrm{~mL} x 2)$, passed through a pad of silica gel $\left(0.5 \mathrm{~g}, \mathrm{Et}_{2} \mathrm{O}\right)$, and concentrated. The residue was purified by PTLC $(10 \mathrm{~cm}$ x $10 \mathrm{~cm}$, hexane/EtOAc = 2/1) to afford bislactone $54(3.11 \mathrm{mg}, 3.27 \mu \mathrm{mol})$ in $55 \%$ yield over 2 steps: white amorphous. $[\alpha]_{\mathrm{D}}{ }^{24}-10.5(c 0.132$, $\mathrm{CHCl}_{3}$ ). IR (film): 2940, 2859, 1717, 1376, 1259, 1116, 1054, 879, 839, $776 \mathrm{~cm}^{-1} .{ }^{1} \mathrm{H}$ NMR (400 MHz, $\left.\mathrm{CDCl}_{3}\right): \delta 9.20(1 \mathrm{H}, \mathrm{d}, J=15.6 \mathrm{~Hz}, \mathrm{H} 7$ '), $8.79(1 \mathrm{H}, \mathrm{d}, J=4.6 \mathrm{~Hz}, \mathrm{H} 6$ '), $8.39(1 \mathrm{H}, \mathrm{dd}, J=8.2,0.9 \mathrm{~Hz}$, H4'), 7.41 (1H, dd, $J=8.2,4.6 \mathrm{~Hz}, \mathrm{H} 5$ '), 6.77 (1H, d, $J=15.6 \mathrm{~Hz}, \mathrm{H} 8$ '), 5.69 (1H, s, H6), 5.06 (1H, $\mathrm{d}, J=11.4 \mathrm{~Hz}, \mathrm{H} 13 \mathrm{a}), 4.97(1 \mathrm{H}, \mathrm{s}, \mathrm{H} 3), 4.84(1 \mathrm{H}, \mathrm{d}, J=12.8 \mathrm{~Hz}, \mathrm{H} 14 \mathrm{a}), 4.81(1 \mathrm{H}, \mathrm{d}, J=6.4 \mathrm{~Hz}$, $\left.\mathrm{OCHaHbOCH}_{3}\right), 4.79(1 \mathrm{H}, \mathrm{d}, J=11.4 \mathrm{~Hz}, \mathrm{H} 13 \mathrm{~b}), 4.74\left(1 \mathrm{H}, \mathrm{d}, J=6.4 \mathrm{~Hz}, \mathrm{OCHaHbOCH}_{3}\right), 4.64(1 \mathrm{H}$, m, H8), 4.23 (1H, d, $J=4.1 \mathrm{~Hz}, \mathrm{H} 1), 4.17$ (1H, d, $J=12.8 \mathrm{~Hz}, \mathrm{H} 14 \mathrm{~b}), 4.01-3.99$ (2H, m, H2, H9), $3.40\left(3 \mathrm{H}, \mathrm{s}, \mathrm{OCH}_{2} \mathrm{OCH}_{3}\right), 3.36(1 \mathrm{H}, \mathrm{s}, \mathrm{OH}), 2.82(1 \mathrm{H}, \mathrm{d}, J=1.8 \mathrm{~Hz}, \mathrm{H} 7), 1.68(3 \mathrm{H}, \mathrm{s}, \mathrm{H} 15), 1.57(3 \mathrm{H}$, $\mathrm{s}, \mathrm{CH}_{3}$ of acetonide), 1.36 (3H, s, H12), 1.31 (3H, s, $\mathrm{CH}_{3}$ of acetonide), 0.98 (9H, s, $t$-Bu of TBS), 0.96 (9H, s, $t$-Bu of TBS), 0.92 (9H, s, $t$-Bu of TBS), 0.26 (3H, s, CH3 of TBS), 0.20 (3H, s, CH3 of TBS), $0.13\left(3 \mathrm{H}, \mathrm{s}, \mathrm{CH}\right.$ of TBS), 0.08 (6H, s, CH3 of TBS x2), 0.05 (3H, s, CH3 of TBS). ${ }^{13} \mathrm{C}\left\{{ }^{1} \mathrm{H}\right\}$ NMR (125 $\left.\mathrm{MHz}_{\mathrm{CDCl}}\right): \delta 166.2,165.9,153.2,152.9,144.2,140.0,125.9,124.6,123.7,108.7,98.3,94.5,83.4$, 82.6, 80.7, 77.3, 75.5, 75.3, 74.1, 71.7, 69.2, 59.7, 56.8, 56.1, 47.8, 27.00 (3C), 26.97, 26.5 (3C), 26.4 (3C), 24.3, 23.8, 19.9, 18.5, 18.3, 18.1, -2.3, -3.9, -4.0, -4.3, -4.6, -4.9. HRMS (ESI-TOF) $[\mathrm{M}+\mathrm{Na}]^{+}$ m/z: Calcd for $\mathrm{C}_{47} \mathrm{H}_{79} \mathrm{NO}_{13} \mathrm{Si}_{3} \mathrm{Na} 972.4751$; Found 972.4730 . 

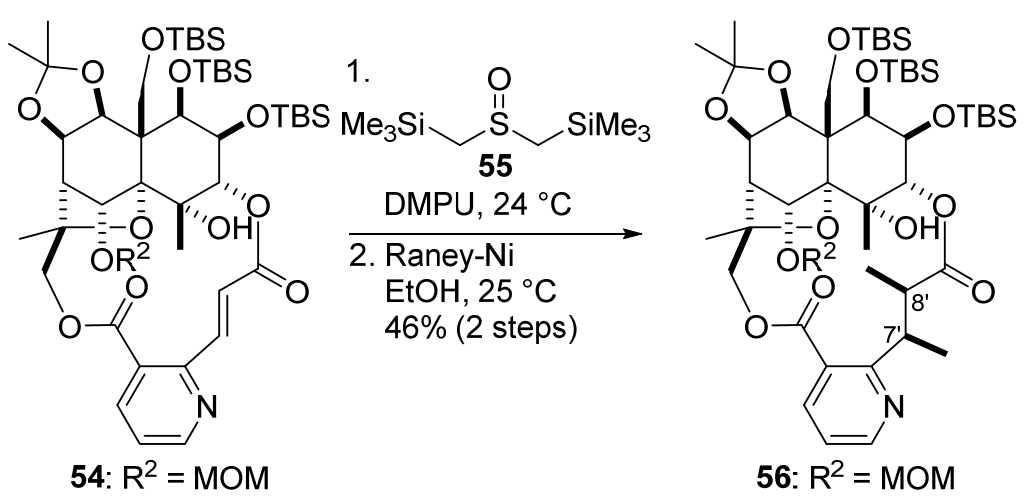

Bislactone 56. A solution of $m$-CPBA (77\% purity, $1.06 \mathrm{~g}, 4.72 \mathrm{mmol})$ in $\mathrm{CH}_{2} \mathrm{Cl}_{2}(10.0 \mathrm{~mL})$ was added dropwise with the dropping funnel to a solution of bis(trimethylsilylmethyl) sulfide (972 $\mathrm{mg}$, $4.71 \mathrm{mmol})$ in $\mathrm{CH}_{2} \mathrm{Cl}_{2}(20.0 \mathrm{~mL})$ at $-78^{\circ} \mathrm{C}$ over $20 \mathrm{~min}$. After the addition was completed, the reaction mixture was warmed to $0{ }^{\circ} \mathrm{C}$ and stirred for $10 \mathrm{~min}$. Then, ice-cold water $(25 \mathrm{~mL})$ was added to the reaction mixture at $0{ }^{\circ} \mathrm{C}$. The organic layer was separated, and the aqueous layer was extracted with $\mathrm{CH}_{2} \mathrm{Cl}_{2}(25 \mathrm{~mL} \times 2)$. The combined organic layers were washed with saturated aqueous $\mathrm{NaHCO}_{3}(25$ $\mathrm{mL} \times 3)$ and bride $(25 \mathrm{~mL})$, dried over $\mathrm{Na}_{2} \mathrm{SO}_{4}$, filtered, and concentrated at $0{ }^{\circ} \mathrm{C}$ to afford bis(trimethylsilylmethyl) sulfoxide (55) (1.04 g, $4.68 \mathrm{mmol})$ as colorless oil, which was used in the next reaction without further purification.

Argon was bubbled through hexane and EtOAc at $24{ }^{\circ} \mathrm{C}$ for $30 \mathrm{~min}$ to remove the residual $\mathrm{O}_{2}$. The solution of the above bis(trimethylsilylmethyl) sulfoxide (55) $(75.1 \mathrm{mg}, 338 \mu \mathrm{mol})$ in DMPU (150 $\mu \mathrm{L})$ was added to a solution of bislactone $54(3.11 \mathrm{mg}, 3.27 \mu \mathrm{mol})$ in DMPU $(400 \mu \mathrm{L})$ at $0{ }^{\circ} \mathrm{C}$. After the reaction mixture was stirred at $24{ }^{\circ} \mathrm{C}$ for $1 \mathrm{~h}, \mathrm{H}_{2} \mathrm{O}(1 \mathrm{~mL})$ was added to the mixture. The resultant mixture was extracted with $\mathrm{Et}_{2} \mathrm{O}(1 \mathrm{~mL} \times 5)$. The combined organic layers were washed with $\mathrm{H}_{2} \mathrm{O}(1$ $\mathrm{mL} \times 2)$, passed through a pad of silica gel $\left(0.5 \mathrm{~g}, \mathrm{Et}_{2} \mathrm{O}\right)$ under argon atmosphere, and concentrated. The residue was purified by flash column chromatography silica gel $(0.5 \mathrm{~g}$, hexane/EtOAc $=80 / 1$ to $3 / 1)$ to afford the crude tetrahydrothiophene $(9.04 \mathrm{mg})$, which was used in the next reaction without further purification. HRMS (ESI-TOF) $[\mathrm{M}+\mathrm{Na}]^{+} \mathrm{m} / \mathrm{z}$ : Calcd for $\mathrm{C}_{49} \mathrm{H}_{83} \mathrm{NO}_{13} \mathrm{SSi}_{3} \mathrm{Na} 1032.4785$; Found 1032.4783.

Raney-Nickel alloy powder (50\% for $\mathrm{Ni}, 3.67 \mathrm{~g})$ was added to $6.2 \mathrm{M}$ aqueous $\mathrm{NaOH}(18.5 \mathrm{~mL})$ at $0{ }^{\circ} \mathrm{C}$ in six separated portions. After the addition was completed, the resultant suspension was stirred at $0{ }^{\circ} \mathrm{C}$ for $30 \mathrm{~min}$, and at $24{ }^{\circ} \mathrm{C}$ for $30 \mathrm{~min}$, and at $75{ }^{\circ} \mathrm{C}$ for $2 \mathrm{~h}$. After the suspension was cooled to $24{ }^{\circ} \mathrm{C}$, the supernatant was removed by decantation. The resultant wet Ni powder was washed with $\mathrm{H}_{2} \mathrm{O}(20 \mathrm{~mL} \times 15)$, EtOH $(99.5 \%, 15 \mathrm{~mL} \times 5)$, and absolute EtOH (15 mL x5). After the final decantation, absolute EtOH ( $5 \mathrm{~mL})$ was added to give a suspension of an activated Raney-Ni.

The above suspension of Raney-Ni in EtOH $(1 \mathrm{~mL})$ was added to the above crude tetrahydrothiophene $\left(9.04 \mathrm{mg}\right.$ ) at $25^{\circ} \mathrm{C}$. After being stirred at $25^{\circ} \mathrm{C}$ for $10 \mathrm{~h}$, the reaction mixture was filtered through a pad of Celite $(0.5 \mathrm{~g})$ with EtOAc $(10 \mathrm{~mL})$ and concentrated. The residue was purified by PTLC (10 
$\mathrm{cm} \times 10 \mathrm{~cm}$, hexane/EtOAc = 2/1) to afford bislactone $\mathbf{5 6}(1.46 \mathrm{mg}, 1.49 \mu \mathrm{mol})$ in $46 \%$ yield over 2 steps. The C7'- and C8'-stereochemistries of $\mathbf{5 6}$ were determined by the X-ray crystallographic analysis (Figure S6, Page S49): colorless plate crystal (recrystallized from hexane/ $\mathrm{CH}_{2} \mathrm{Cl}_{2}$ ). m.p. 208$209{ }^{\circ} \mathrm{C} .[\alpha]_{\mathrm{D}}^{26}-29.3$ (c 0.0730, $\left.\mathrm{CHCl}_{3}\right)$. IR (film): 2937, 2859, 1725, 1374, 1254, 1115, 1064, 882, 839, $774 \mathrm{~cm}^{-1} .{ }^{1} \mathrm{H}$ NMR (400 MHz, $\left.\mathrm{CDCl}_{3}\right): \delta 8.67(1 \mathrm{H}, \mathrm{d}, J=4.6 \mathrm{~Hz}, \mathrm{H} 6$ '), $8.14(1 \mathrm{H}, \mathrm{d}, J=7.3 \mathrm{~Hz}$, H4'), 7.20 (1H, dd, $J=7.3,4.6$ Hz, H5'), 5.67 (1H, s, H6), 5.59 (1H, d, $J=11.5$ Hz, H13a), 4.82-4.74 $\left(4 \mathrm{H}, \mathrm{m}, \mathrm{H} 14 \mathrm{a}, \mathrm{H} 7\right.$ ', $\left.\mathrm{OCH}_{2} \mathrm{OCH}_{3}\right), 4.71(1 \mathrm{H}, \mathrm{d}, J=1.4 \mathrm{~Hz}, \mathrm{H} 3), 4.66(1 \mathrm{H}, \mathrm{d}, J=6.8 \mathrm{~Hz}, \mathrm{H} 8), 4.21(1 \mathrm{H}$, $\mathrm{d}, J=3.7 \mathrm{~Hz}, \mathrm{H1}), 4.14(1 \mathrm{H}, \mathrm{d}, J=12.8 \mathrm{~Hz}, \mathrm{H} 14 \mathrm{~b}), 4.00(1 \mathrm{H}, \mathrm{d}, J=11.5 \mathrm{~Hz}, \mathrm{H} 13 \mathrm{~b}), 3.99(1 \mathrm{H}, \mathrm{d}, J=$ $6.8 \mathrm{~Hz}, \mathrm{H} 9), 3.81(1 \mathrm{H}, \mathrm{m}, \mathrm{H} 2), 3.53(1 \mathrm{H}, \mathrm{s}, \mathrm{OH}), 3.46\left(3 \mathrm{H}, \mathrm{s}, \mathrm{OCH}_{2} \mathrm{OCH}_{3}\right), 3.35(1 \mathrm{H}, \mathrm{qd}, J=7.3,7.3$ Hz, H8'), 2.81 (1H, s, H7), 1.59 (3H, s, H15), 1.57 (3H, s, CH3 of acetonide), 1.51 (3H, s, H12), 1.32 (3H, s, $\mathrm{CH}_{3}$ of acetonide), 1.29 (3H, d, $J=7.3 \mathrm{~Hz}, \mathrm{H} 10$ '), 1.22 (3H, d, $J=7.3 \mathrm{~Hz}, \mathrm{H} 9$ '), 0.94 (9H, s, $t$ Bu of TBS), 0.93 (18H, s, $t$-Bu of TBS x2), 0.18 (3H, s, CH3 of TBS), 0.13 (3H, s, CH3 of TBS), 0.12 (3H, s, CH3 of TBS), 0.10 (3H, s, $\mathrm{CH}_{3}$ of TBS), 0.06 (3H, s, CH3 of TBS), 0.05 (3H, s, $\mathrm{CH}_{3}$ of TBS). ${ }^{13} \mathrm{C}\left\{{ }^{1} \mathrm{H}\right\}$ NMR $\left(125 \mathrm{MHz}, \mathrm{CDCl}_{3}\right): \delta 174.2,167.9,163.8,151.7,139.3,125.8,121.2,108.5,98.5,94.0$, 83.6, 81.1, 79.6, 77.9, 75.6, 75.0, 74.5, 70.8, 69.5, 59.8, 56.9, 56.2, 48.5, 44.2, 38.5, 27.0 (4C), 26.4 (3C), 26.3 (3C), 23.7 (2C), 20.8, 18.5, 18.4, 18.0, 17.5, 14.6, -2.2, -4.0, -4.3, -4.4, -4.6, -4.8. HRMS $(\mathrm{ESI}-\mathrm{TOF})[\mathrm{M}+\mathrm{Na}]^{+} \mathrm{m} / \mathrm{z}$ : Calcd for $\mathrm{C}_{49} \mathrm{H}_{85} \mathrm{NO}_{13} \mathrm{Si}_{3} \mathrm{Na}$ 1002.5221; Found 1002.5218.

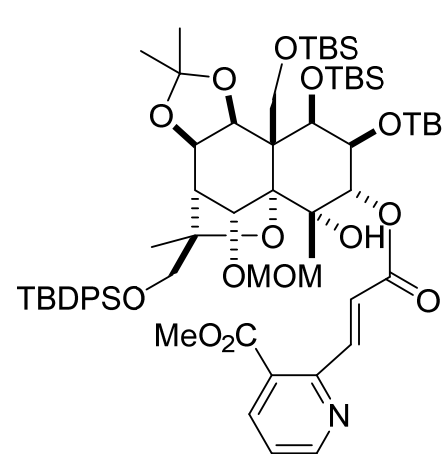

51

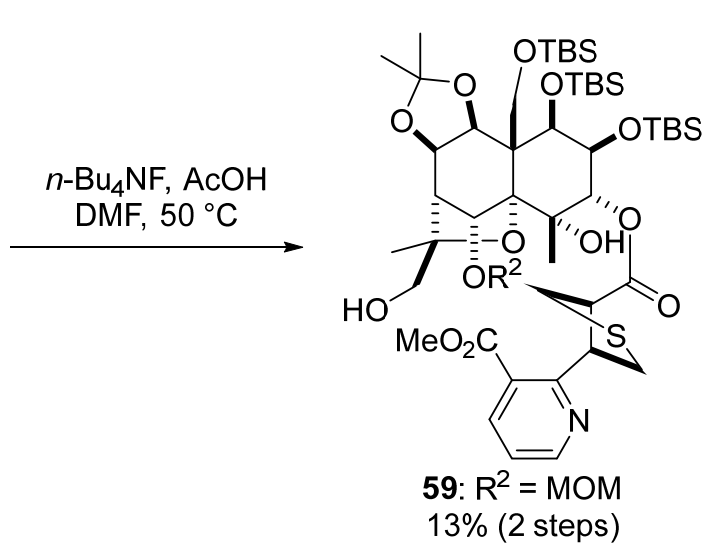

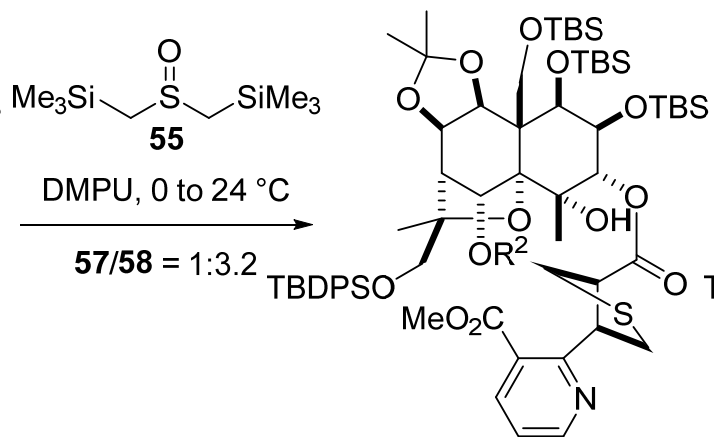

57: $\mathrm{R}^{2}=\mathrm{MOM}$

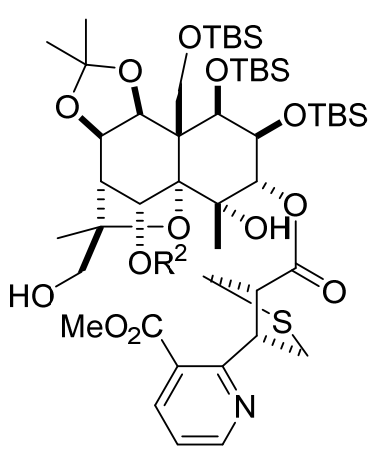

60: $\mathrm{R}^{2}=\mathrm{MOM}$ $48 \%$ (2 steps)

Alcohols 59 and 60. The solution of bis(trimethylsilylmethyl) sulfoxide (55) (Page S34, 263 mg, 1.18 mmol) in DMPU $(600 \mu \mathrm{L})$ was added to a solution of ester $\mathbf{5 1}(70.1 \mathrm{mg}, 57.4 \mu \mathrm{mol})$ in DMPU (1.50 
$\mathrm{mL})$ at $0{ }^{\circ} \mathrm{C}$. After the reaction mixture was stirred at $24{ }^{\circ} \mathrm{C}$ for $1 \mathrm{~h}, \mathrm{H}_{2} \mathrm{O}(2 \mathrm{~mL})$ was added to the mixture. The resultant mixture was extracted with $\mathrm{Et}_{2} \mathrm{O}(2 \mathrm{~mL} \times 5)$. The combined organic layers were washed with $\mathrm{H}_{2} \mathrm{O}$ ( $2 \mathrm{~mL}$ x2), dried over $\mathrm{Na}_{2} \mathrm{SO}_{4}$, filtered, and concentrated. The residue was purified by automated flash column chromatography using the prepacked silica gel $(7 \mathrm{~g}$, hexane/EtOAc $=99 / 1$ to 78/22) to afford a 1:3.2 diastereomeric mixture of the crude esters $\mathbf{5 7}$ and $\mathbf{5 8}$ (74.0 $\mathrm{mg}$, Page S116), which was used in the next reaction without further purification. HRMS (ESI-TOF) $[\mathrm{M}+\mathrm{Na}]^{+} \mathrm{m} / \mathrm{z}$ : Calcd for $\mathrm{C}_{66} \mathrm{H}_{105} \mathrm{NO}_{14} \mathrm{SSi}_{4} \mathrm{Na} 1302.6225$; Found 1302.6248.

Argon was bubbled through DMF, hexane, and EtOAc at $25^{\circ} \mathrm{C}$ for $30 \mathrm{~min}$ to remove the residual $\mathrm{O}_{2}$. $n$-Bu4NF (1 M THF solution) was degassed by freeze-thaw procedure (x3). A mixture of AcOH (36.0 $\mu \mathrm{L}, 0.630 \mathrm{mmol}$ ) and $n$-Bu4NF (1 M THF solution, $570 \mu \mathrm{L}, 570 \mu \mathrm{mol})$ in DMF (400 $\mu \mathrm{L}$ ) was added to a solution of the above crude esters 57 and $\mathbf{5 8}(74.0 \mathrm{mg})$ in DMF $(1.00 \mathrm{~mL})$ at $25{ }^{\circ} \mathrm{C}$ via cannula. After the reaction mixture was stirred at $50{ }^{\circ} \mathrm{C}$ for $24 \mathrm{~h}, \mathrm{pH} 7$ phosphate buffer $(1.5 \mathrm{~mL})$ was added to the mixture at $0{ }^{\circ} \mathrm{C}$. The resultant mixture was extracted with $\mathrm{Et}_{2} \mathrm{O}(3 \mathrm{~mL}$ x5). The combined organic layers were washed with $\mathrm{H}_{2} \mathrm{O}\left(3 \mathrm{~mL}\right.$ x2), dried over $\mathrm{Na}_{2} \mathrm{SO}_{4}$, filtered, and concentrated. The residue was purified by flash column chromatography on silica gel $(3 \mathrm{~g}$, hexane/EtOAc $=5 / 1$ to $1 / 1)$ under argon atmosphere to afford alcohols $59(7.73 \mathrm{mg}, 7.41 \mu \mathrm{mol})$ and $\mathbf{6 0}(29.0 \mathrm{mg}, 27.8 \mu \mathrm{mol})$ in $13 \%$ and $48 \%$ yields over 2 steps, respectively.

59: white amorphous. $[\alpha]_{\mathrm{D}}{ }^{25}-6.23\left(c 0.388, \mathrm{CHCl}_{3}\right)$. IR (film): $3466,2938,2859,1730,1256,1114$, 1084, 1051, 839, $776 \mathrm{~cm}^{-1} .{ }^{1} \mathrm{H}$ NMR (400 MHz, $\left.\mathrm{CDCl}_{3}\right): \delta 8.64(1 \mathrm{H}, \mathrm{dd}, J=4.6,1.8 \mathrm{~Hz}, \mathrm{H6}$ '), 8.13 (1H, dd, $J=8.2,1.8 \mathrm{~Hz}, \mathrm{H} 4$ ') 7.20 (1H, dd, $J=8.2$, 4.6 Hz, H5'), 5.62 (1H, s, H6), 4.89 (1H, d, $J=$ $2.3 \mathrm{~Hz}, \mathrm{H} 3), 4.75\left(1 \mathrm{H}, \mathrm{m}, \mathrm{H} 7\right.$ ') $4.74\left(1 \mathrm{H}, \mathrm{d}, J=6.4 \mathrm{~Hz}, \mathrm{OCHaHbOCH}_{3}\right), 4.69(1 \mathrm{H}, \mathrm{d}, J=6.4 \mathrm{~Hz}$, $\left.\mathrm{OCHaHbOCH}_{3}\right), 4.65(1 \mathrm{H}, \mathrm{d}, J=12.4 \mathrm{~Hz}, \mathrm{H} 14 \mathrm{a}), 4.61(1 \mathrm{H}, \mathrm{dd}, J=7.3,2.3 \mathrm{~Hz}, \mathrm{H} 8), 4.14-4.11(2 \mathrm{H}$, m, H1, H13a), 4.08 (1H, d, $J=12.4 \mathrm{~Hz}, \mathrm{H} 14 \mathrm{~b}), 3.939$ (3H, s, $\left.\mathrm{CO}_{2} \mathrm{CH}_{3}\right), 3.938(1 \mathrm{H}, \mathrm{d}, J=7.3 \mathrm{~Hz}, \mathrm{H} 9)$, 3.83 (1H, m, H8'), 3.53-3.49 (2H, m, H2, OH), 3.41-3.30 (4H, m, H13b, H9'a, H10'a, OH), 3.37 (3H, s, $\left.\mathrm{OCH}_{2} \mathrm{OCH}_{3}\right), 3.17(1 \mathrm{H}, \mathrm{dd}, J=10.6,10.6 \mathrm{~Hz}, \mathrm{H} 10$ 'b), 3.12 (1H, dd, $J=9.6,9.6 \mathrm{~Hz}, \mathrm{H} 9$ 'b), 2.64 $(1 \mathrm{H}, \mathrm{d}, J=2.3 \mathrm{~Hz}, \mathrm{H} 7), 1.63$ (3H, s, H15), 1.53 (3H, s, CH3 of acetonide), 1.40 (3H, s, H12), 1.29 (3H, s, $\mathrm{CH}_{3}$ of acetonide), 0.92 (9H, s, $t$-Bu of TBS), 0.91 (9H, s, $t$-Bu of TBS), 0.88 (9H, s, $t$-Bu of TBS), 0.12 ( $3 \mathrm{H}, \mathrm{s}, \mathrm{CH}_{3}$ of TBS), 0.07 (3H, s, CH3 of TBS), 0.03 (3H, s, $\mathrm{CH}_{3}$ of TBS), -0.016 (3H, s, $\mathrm{CH}_{3}$ of TBS $),-0.024(3 \mathrm{H}, \mathrm{s}, \mathrm{CH} 3$ of TBS $),-0.09$ (3H, s, CH3 of TBS). ${ }^{13} \mathrm{C}\left\{{ }^{1} \mathrm{H}\right\} \mathrm{NMR}\left(125 \mathrm{MHz}, \mathrm{CDCl}_{3}\right): \delta$ 172.0, 166.7, 160.5, 151.8, 138.5, 126.4, 121.4, 108.4, 98.0, 92.4, 83.9, 82.6, 79.4, 77.9, 75.8, 75.6, 74.4, 71.3, 65.9, 59.8, 56.8, 55.9, 53.5, 52.7, 50.2, 46.9, 35.9, 33.1, 27.0 (4C), 26.40 (3C), 26.37 (3C), $25.1,23.7,21.2,18.42,18.39,18.1,-2.2,-4.0,-4.3,-4.57,-4.62,-4.9$. HRMS (ESI-TOF) $[\mathrm{M}+\mathrm{Na}]^{+}$ $\mathrm{m} / \mathrm{z}$ : Calcd for $\mathrm{C}_{50} \mathrm{H}_{87} \mathrm{NO}_{14} \mathrm{SSi}_{3} \mathrm{Na}$ 1064.5047; Found 1064.5047.

60: white amorphous. $[\alpha]_{\mathrm{D}}^{27}+39.2\left(c 0.725, \mathrm{CHCl}_{3}\right)$. IR (film): 3501, 2944, 2860, 1729, 1257, 1206, $1114,880,839,776 \mathrm{~cm}^{-1} .{ }^{1} \mathrm{H}$ NMR (500 MHz, $\left.\mathrm{CDCl}_{3}\right)$ : $\delta 8.68(1 \mathrm{H}, \mathrm{dd}, J=4.6,1.7 \mathrm{~Hz}, \mathrm{H} 6$ '), $8.16(1 \mathrm{H}$, 
dd, $\left.J=8.0,1.7 \mathrm{~Hz}, \mathrm{H} 4^{\prime}\right), 7.22$ (1H, dd, $J=8.0,4.6 \mathrm{~Hz}, \mathrm{H} 5$ '), $5.61(1 \mathrm{H}, \mathrm{s}, \mathrm{H6}), 4.92(1 \mathrm{H}, \mathrm{d}, J=2.3 \mathrm{~Hz}$, H3), 4.87 (1H, ddd, $J=6.9,6.9,6.9 \mathrm{~Hz}, \mathrm{H} 7$ ') $4.76\left(1 \mathrm{H}, \mathrm{d}, J=6.3 \mathrm{~Hz}, \mathrm{OCHaHbOCH}_{3}\right), 4.70$ (1H, d, $\left.J=6.3 \mathrm{~Hz}, \mathrm{OCHaHbOCH}_{3}\right), 4.69(1 \mathrm{H}, \mathrm{d}, J=12.6 \mathrm{~Hz}, \mathrm{H} 14 \mathrm{a}), 4.64(1 \mathrm{H}, \mathrm{dd}, J=7.4,2.3 \mathrm{~Hz}, \mathrm{H} 8), 4.25-$ $4.22(2 \mathrm{H}, \mathrm{m}, \mathrm{H} 1, \mathrm{H} 13 \mathrm{a}), 4.12(1 \mathrm{H}, \mathrm{d}, J=12.6 \mathrm{~Hz}, \mathrm{H} 14 \mathrm{~b}), 3.97$ (1H, d, $J=7.4 \mathrm{~Hz}, \mathrm{H} 9), 3.92$ (3H, s, $\left.\mathrm{CO}_{2} \mathrm{CH}_{3}\right), 3.86(1 \mathrm{H}, \mathrm{ddd}, J=6.9,6.9,6.9 \mathrm{~Hz}, \mathrm{H} 8$ '), $3.79(1 \mathrm{H}, \mathrm{dd}, J=4.6,2.3 \mathrm{~Hz}, \mathrm{H} 2), 3.61(1 \mathrm{H}, \mathrm{d}, J$ $=9.2 \mathrm{~Hz}, \mathrm{OH}), 3.50(1 \mathrm{H}, \mathrm{s}, \mathrm{OH}), 3.38\left(3 \mathrm{H}, \mathrm{s}, \mathrm{OCH}_{2} \mathrm{OCH}_{3}\right), 3.36(1 \mathrm{H}, \mathrm{m}, \mathrm{H} 9$ 'a), 3.26-3.24 (3H, m, H13b, H10'a, H10'b), 3.07 (1H, dd, $J=10.3,6.9$ Hz, H9'b), 2.62 (1H, d, $J=2.3$ Hz, H7), 1.66 (3H, s, H15), 1.54 (3H, s, CH3 of acetonide), 1.43 (3H, s, H12), 1.30 (3H, s, CH3 of acetonide), 0.934 (9H, s, $t$-Bu of TBS), 0.931 (9H, s, $t$-Bu of TBS), 0.92 (9H, s, $t$-Bu of TBS), 0.18 (3H, s, CH3 of TBS), 0.12 ( $3 \mathrm{H}, \mathrm{s}, \mathrm{CH}_{3}$ of TBS), 0.11 (3H, s, $\mathrm{CH}_{3}$ of TBS), 0.08 (3H, s, $\mathrm{CH}_{3}$ of TBS), 0.05 (6H, s, $\mathrm{CH}_{3}$ of TBS x2). ${ }^{13} \mathrm{C}\left\{{ }^{1} \mathrm{H}\right\}$ NMR $\left(125 \mathrm{MHz}, \mathrm{CDCl}_{3}\right): \delta 171.7,166.6,160.9,152.1,138.6,125.4,121.6,108.3,98.0,92.3$, 84.1, 82.6, 79.1, 77.9, 75.9, 75.3, 74.5, 71.5, 65.7, 60.0, 56.8, 56.0, 53.1, 52.5, 49.2, 47.2, 35.4, 32.9, 27.0 (4C), 26.5 (3C), 26.4 (3C), 25.3, 23.6, 20.9, 18.47, 18.45, 18.1, -2.0, -4.0, -4.3, -4.4, -4.5, -4.7. HRMS (ESI-TOF) $[\mathrm{M}+\mathrm{Na}]^{+} \mathrm{m} / \mathrm{z}$ : Calcd for $\mathrm{C}_{50} \mathrm{H}_{87} \mathrm{NO}_{14} \mathrm{SSi}_{3} \mathrm{Na}$ 1064.5047; Found 1064.5056.

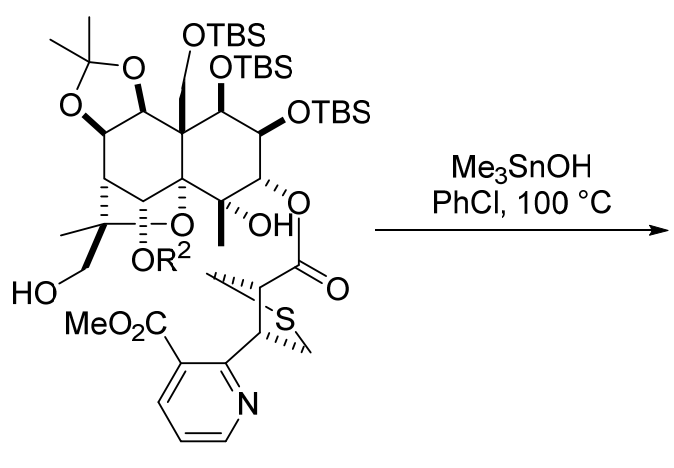

60: $R^{2}=$ MOM

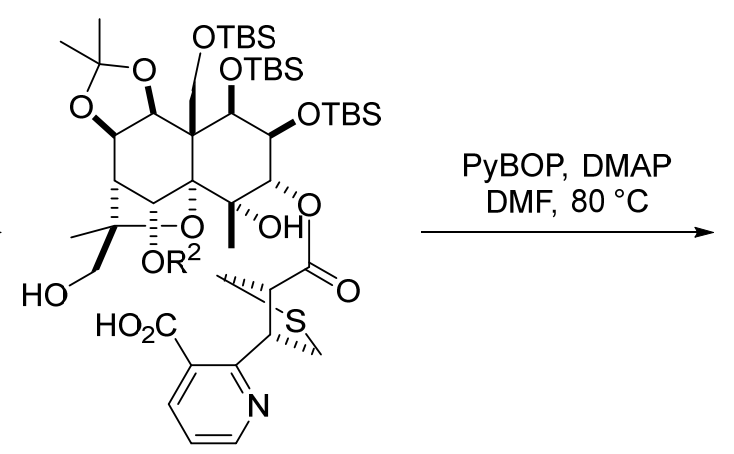

61: $R^{2}=$ MOM
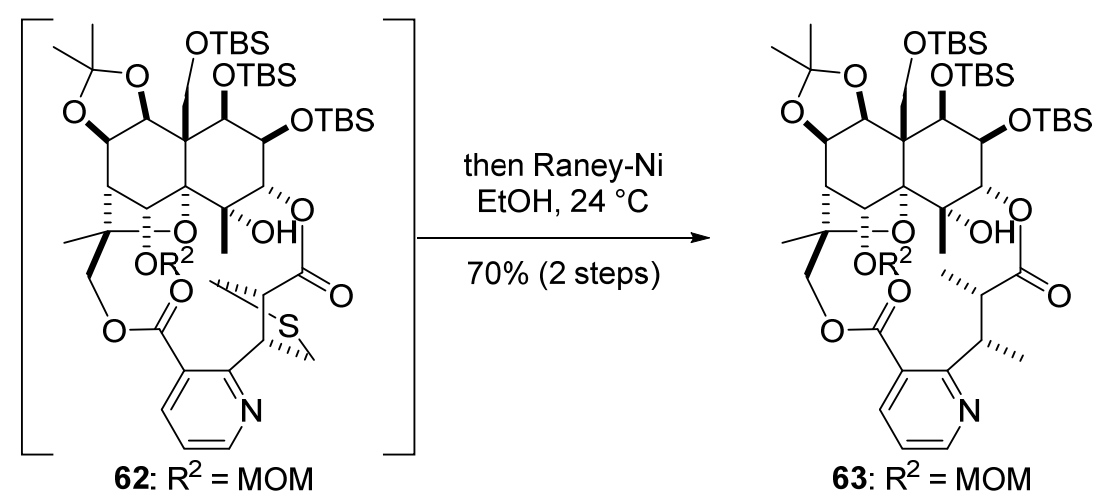

Bislactone 63. Argon was bubbled through $\mathrm{CHCl}_{3}$ and $\mathrm{MeOH}$ at $23{ }^{\circ} \mathrm{C}$ for $30 \mathrm{~min}$ to remove the residual $\mathrm{O}_{2} . \mathrm{Me} 3 \mathrm{SnOH}(79.1 \mathrm{mg}, 437 \mu \mathrm{mol})$ was added to a solution of alcohol $\mathbf{6 0}(11.4 \mathrm{mg}, 10.9$ $\mu \mathrm{mol})$ in $\mathrm{PhCl}(1.10 \mathrm{~mL})$ at $23{ }^{\circ} \mathrm{C}$. The reaction mixture was degassed by freeze-thaw procedure (x3) and stirred at $100{ }^{\circ} \mathrm{C}$ for $24 \mathrm{~h}$. After being cooled to $23{ }^{\circ} \mathrm{C}$, the reaction mixture was directly passed 
through a pad of silica gel $\left(3 \mathrm{~g}, \mathrm{CHCl}_{3} / \mathrm{MeOH}=1 / 0\right.$ to $\left.20 / 1\right)$ under argon atmosphere and concentrated to afford the crude seco acid $\mathbf{6 1}(14.3 \mathrm{mg})$, which was used in the next reaction without further purification. HRMS (ESI-TOF) [M-H] ${ }^{-}$m/z: Calcd for $\mathrm{C}_{49} \mathrm{H}_{84} \mathrm{NO}_{14} \mathrm{SSi}_{3}$ 1026.4926; Found 1026.4898. The above crude seco acid 61 (14.3 mg) was azeotroped with toluene (2 mL x3) and dissolved in DMF (1.10 mL). DMAP (5.4 mg, $44 \mu \mathrm{mol})$ and PyBOP (11.6 mg, $22.3 \mathrm{mmol}$ ) were successively added to the solution of 61 in DMF at $23{ }^{\circ} \mathrm{C}$. The reaction mixture was degassed by freeze-thaw procedure (x3). After the reaction mixture was stirred at $80{ }^{\circ} \mathrm{C}$ for $6 \mathrm{~h}$, the suspension of Raney-Ni in EtOH (Page S34, $600 \mu \mathrm{L}$ ) was added to the reaction mixture at $24{ }^{\circ} \mathrm{C}$. After being stirred at $24{ }^{\circ} \mathrm{C}$ for $3 \mathrm{~h}$, the reaction mixture was filtered through a pad of Celite $(0.5 \mathrm{~g})$ with EtOAc $(15 \mathrm{~mL})$ and concentrated. The residue was purified by PTLC $(20 \mathrm{~cm} \times 10 \mathrm{~cm}, 10 \mathrm{~cm}$ x $10 \mathrm{~cm}$, hexane/EtOAc = 1/1) to afford bislactone 63 $(7.43 \mathrm{mg}, 7.58 \mu \mathrm{mol})$ in $70 \%$ yield over 2 steps. The C7'- and C8'-stereochemistries of $\mathbf{6 3}$ were determined by the X-ray crystallographic analysis (Figure S7, Page S50): colorless block crystal (recrystallized from $\mathrm{CH}_{2} \mathrm{Cl}_{2}$ ). m.p. $253-255{ }^{\circ} \mathrm{C} .[\alpha]_{\mathrm{D}}{ }^{20}+49.4\left(c 0.410, \mathrm{CHCl}_{3}\right)$. IR (film): 3553, 2940, $2860,1728,1257,1114,1065,1010,882,840 \mathrm{~cm}^{-1} .{ }^{1} \mathrm{H} \mathrm{NMR}\left(500 \mathrm{MHz}, \mathrm{CDCl}_{3}\right): \delta 8.66(1 \mathrm{H}, \mathrm{dd}, J=$ 4.6, 1.7 Hz, H6'), 7.95 (1H, dd, J = 7.5, 1.7 Hz, H4'), 7.21 (1H, dd, J = 7.5, 4.6 Hz, H5'), 5.61 (1H, s, H6), $5.38(1 \mathrm{H}, \mathrm{d}, J=11.5 \mathrm{~Hz}, \mathrm{H} 13 \mathrm{a}), 4.93(1 \mathrm{H}, \mathrm{d}, J=2.3 \mathrm{~Hz}, \mathrm{H} 3), 4.82(1 \mathrm{H}, \mathrm{d}, J=6.9 \mathrm{~Hz}$, $\left.\mathrm{OCHaHbOCH}_{3}\right), 4.760\left(1 \mathrm{H}, \mathrm{d}, J=6.9 \mathrm{~Hz}, \mathrm{OCHaHbOCH}_{3}\right), 4.759(1 \mathrm{H}, \mathrm{d}, J=12.0 \mathrm{~Hz}, \mathrm{H} 14 \mathrm{a}), 4.64$ $(1 \mathrm{H}, \mathrm{dd}, J=6.9,2.3 \mathrm{~Hz}, \mathrm{H} 8), 4.21(1 \mathrm{H}, \mathrm{qd}, J=6.9,1.8 \mathrm{~Hz}, \mathrm{H} 7$ '), $4.16(1 \mathrm{H}, \mathrm{d}, J=4.0 \mathrm{~Hz}, \mathrm{H1}), 4.13$ $(1 \mathrm{H}, \mathrm{d}, J=11.5 \mathrm{~Hz}, \mathrm{H} 13 \mathrm{~b}), 4.12(1 \mathrm{H}, \mathrm{d}, J=12.0 \mathrm{~Hz}, \mathrm{H} 14 \mathrm{~b}), 3.94(1 \mathrm{H}, \mathrm{d}, J=6.9 \mathrm{~Hz}, \mathrm{H} 9), 3.84(1 \mathrm{H}$, $\mathrm{dd}, J=4.0,2.3 \mathrm{~Hz}, \mathrm{H} 2), 3.58(1 \mathrm{H}, \mathrm{s}, \mathrm{OH}), 3.45\left(3 \mathrm{H}, \mathrm{s}, \mathrm{OCH}_{2} \mathrm{OCH}_{3}\right), 2.87(1 \mathrm{H}, \mathrm{qd}, J=6.9,1.8 \mathrm{~Hz}$, H8'), $2.78(1 \mathrm{H}, \mathrm{d}, J=2.3 \mathrm{~Hz}, \mathrm{H} 7), 1.68(3 \mathrm{H}, \mathrm{s}, \mathrm{H} 15), 1.56(3 \mathrm{H}, \mathrm{s}, \mathrm{CH}$ of acetonide), 1.39 (3H, d, $J=$ $6.9 \mathrm{~Hz}, \mathrm{H} 10$ ') 1.36 (3H, s, H12), 1.302 (3H, s, CH3 of acetonide), 1.300 (3H, d, J = 6.9 Hz, H9'), 0.963 (9H, s, $t$-Bu of TBS), 0.956 (9H, s, $t$-Bu of TBS), 0.91 (9H, s, $t$-Bu of TBS), 0.23 (3H, s, CH $H_{3}$ of TBS), 0.15 (3H, s, CH3 of TBS), 0.11 (3H, s, CH3 of TBS), 0.07 (3H, s, CH3 of TBS), 0.06 (3H, s, $\mathrm{CH}_{3}$ of TBS), 0.01 (3H, s, $\mathrm{CH}$ of TBS). ${ }^{13} \mathrm{C}\left\{{ }^{1} \mathrm{H}\right\} \mathrm{NMR}\left(125 \mathrm{MHz}, \mathrm{CDCl}_{3}\right): \delta 175.0,168.7,163.2,150.7$, 137.8, 127.2, 120.9, 108.5, 98.4, 94.1, 83.5, 81.5, 79.2, 77.6, 75.7, 75.3, 74.5, 71.1, 70.1, 59.8, 56.9, 56.1, 48.4, 43.4, 38.0, 27.0 (4C), 26.5 (3C), 26.4 (3C), 24.5, 23.7, 19.4, 18.5, 18.3, 18.1, 13.9, 10.3, $2.4,-3.99,-4.01,-4.3,-4.6,-4.9$. HRMS (ESI-TOF) $[\mathrm{M}+\mathrm{Na}]^{+} \mathrm{m} / \mathrm{z}$ : Calcd for $\mathrm{C}_{49} \mathrm{H}_{85} \mathrm{NO}_{13} \mathrm{Si}_{3} \mathrm{Na}$ 1002.5221 ; Found 1002.5229. 

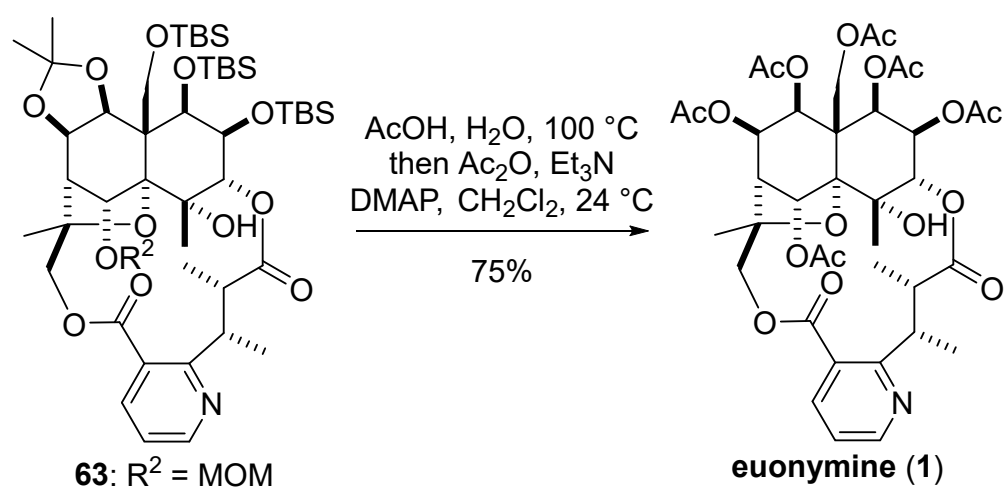

Euonymine (1) [CAS: 33458-82-1]. A mixture of bislactone 63 (8.28 mg, $8.45 \mu \mathrm{mol})$, AcOH (1.20 $\mathrm{mL})$, and $\mathrm{H}_{2} \mathrm{O}(600 \mu \mathrm{L})$ was stirred at $100{ }^{\circ} \mathrm{C}$ for $16 \mathrm{~h}$. After being cooled to $24{ }^{\circ} \mathrm{C}$, the reaction mixture was azeotropically dried with toluene $(2 \mathrm{~mL} \times 5)$. The residue was dissolved in $\mathrm{CH}_{2} \mathrm{Cl}_{2}(1.70$ $\mathrm{mL})$. Et $3 \mathrm{~N}(141 \mu \mathrm{L}, 1.01 \mathrm{mmol}), \mathrm{Ac}_{2} \mathrm{O}(80.0 \mu \mathrm{L}, 847 \mu \mathrm{mol})$, and DMAP $(0.61 \mathrm{mg}, 5.0 \mu \mathrm{mol})$ were successively added to the mixture at $24{ }^{\circ} \mathrm{C}$. After the reaction mixture was stirred at $24{ }^{\circ} \mathrm{C}$ for $36 \mathrm{~h}$, saturated aqueous $\mathrm{NH}_{4} \mathrm{Cl}(2 \mathrm{~mL})$ was added to the mixture at $0{ }^{\circ} \mathrm{C}$. The resultant mixture was extracted with EtOAc $(2 \mathrm{~mL} \times 5)$. The combined organic layers were dried over $\mathrm{Na}_{2} \mathrm{SO}_{4}$, filtered, and concentrated. The residue was passed through a pad of silica gel (0.5 g, EtOAc) and concentrated. The residue was purified by PTLC $\left(20 \mathrm{~cm} \mathrm{x} 10 \mathrm{~cm}, \mathrm{CH}_{2} \mathrm{Cl}_{2} /\right.$ acetone $=8 / 1$, developed twice $)$, to afford euonymine (1) $(5.10 \mathrm{mg}, 6.34 \mu \mathrm{mol})$ in $75 \%$ yield: white amorphous. $[\alpha]_{\mathrm{D}}{ }^{25}-21.3\left(c 0.247, \mathrm{CHCl}_{3}\right)$. IR (film): 3496, 2978, 2860, 1748, 1572, 1434, 1372, 1231, 1117, $1054 \mathrm{~cm}^{-1} .{ }^{1} \mathrm{H}$ NMR (400 MHz, $\left.\mathrm{C}_{6} \mathrm{D}_{6}\right): \delta 8.33$ (1H, dd, $J=4.6,1.8 \mathrm{~Hz}, \mathrm{H6}$ ') 7.55 (1H, dd, $J=7.8,1.8 \mathrm{~Hz}, \mathrm{H} 4$ ') 7.19 (1H, s, H6), 6.52 $(1 \mathrm{H}, \mathrm{dd}, J=7.8,4.6 \mathrm{~Hz}, \mathrm{H} 5$ ') $6.02(1 \mathrm{H}, \mathrm{d}, J=3.7 \mathrm{~Hz}, \mathrm{H} 1), 5.87$ (1H, d, $J=11.4 \mathrm{~Hz}, \mathrm{H} 13 \mathrm{a}), 5.78(1 \mathrm{H}$, $\mathrm{dd}, J=3.7,2.3 \mathrm{~Hz}, \mathrm{H} 2), 5.67(1 \mathrm{H}, \mathrm{d}, J=6.0 \mathrm{~Hz}, \mathrm{H} 9), 5.64(1 \mathrm{H}, \mathrm{dd}, J=6.0,3.6 \mathrm{~Hz}, \mathrm{H} 8), 5.46$ (1H, d, $J=13.2 \mathrm{~Hz}, \mathrm{H} 14 \mathrm{a}), 5.15$ (1H, d, $J=2.3 \mathrm{~Hz}, \mathrm{H} 3), 4.99$ (1H, q, $J=7.4 \mathrm{~Hz}, \mathrm{H} 7$ '), 4.77 (1H, d, $J=13.2$ Hz, H14b), 4.68 (1H, s, OH), 3.08 (1H, d, J=11.4 Hz, H13b), 2.72 (1H, q, J = 7.3 Hz, H8'), 2.23 (3H, s, $\mathrm{CH}_{3}$ of Ac), 1.91 (3H, s, $\mathrm{CH}_{3}$ of $\left.\mathrm{Ac}\right), 1.88\left(3 \mathrm{H}, \mathrm{s}, \mathrm{CH}_{3}\right.$ of $\left.\mathrm{Ac}\right), 1.75(3 \mathrm{H}, \mathrm{s}, \mathrm{H} 15), 1.70(1 \mathrm{H}, \mathrm{d}, J=3.6$ $\mathrm{Hz}, \mathrm{H} 7), 1.67$ (3H, s, $\mathrm{CH}_{3}$ of Ac), 1.66 (3H, s, $\mathrm{CH}_{3}$ of Ac), $1.61\left(3 \mathrm{H}, \mathrm{s}, \mathrm{CH}_{3}\right.$ of Ac), 1.57 (3H, d, $J=$ $7.4 \mathrm{~Hz}, \mathrm{H} 9$ '), 1.31 (3H, s, H12), 1.27 (3H, d, $\left.J=7.3 \mathrm{~Hz}, \mathrm{H} 10{ }^{\prime}\right) .{ }^{13} \mathrm{C}\left\{{ }^{1} \mathrm{H}\right\}$ NMR (125 MHz, $\left.\mathrm{C}_{6} \mathrm{D}_{6}\right): \delta$ 174.1, 169.8, 169.7, 169.5, 169.0, 168.9, 168.55, 168.46, 165.6, 151.5, 137.3, 125.5, 120.8, 94.5, 84.1, 76.5, 73.9, 73.8, 71.0, 70.9, 69.7, 69.2 (2C), 60.2, 52.6, 50.5, 45.2, 37.1, 23.4, 21.2 (2C), 20.7, 20.3, 20.2, 20.1, 17.9, 12.3, 9.5. HRMS (ESI-TOF) $[\mathrm{M}+\mathrm{Na}]^{+} \mathrm{m} / \mathrm{z}$ : Calcd for $\mathrm{C}_{38} \mathrm{H}_{47} \mathrm{NO}_{18} \mathrm{Na}$ 828.2685; Found 828.2676. 
2. Comparison of ${ }^{1} \mathrm{H}$ and ${ }^{13} \mathrm{C}\left\{{ }^{1} \mathrm{H}\right\}$ NMR data of natural, reported and synthetic 1 and 2

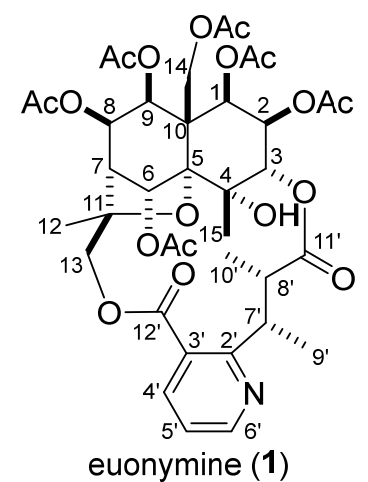

Table S2. ${ }^{1} \mathrm{H}$ and ${ }^{13} \mathrm{C}\left\{{ }^{1} \mathrm{H}\right\}$ NMR data of natural ${ }^{a}$ and synthetic euonymine (1) in $\mathrm{C}_{6} \mathrm{D}_{6}$.

\begin{tabular}{|c|c|c|c|c|}
\hline \multirow[b]{2}{*}{ No. } & \multicolumn{2}{|c|}{ natural $1(500,125 \mathrm{MHz})^{a}$} & \multicolumn{2}{|c|}{ synthetic $\mathbf{1}(400,125 \mathrm{MHz})$} \\
\hline & $\mathrm{H}[\delta$, multi, $J(\mathrm{~Hz})]$ & ${ }^{13} \mathrm{C}\left\{{ }^{1} \mathrm{H}\right\}(\delta)$ & ${ }^{1} \mathrm{H}[\delta$, multi, $J(\mathrm{~Hz})]$ & ${ }^{13} \mathrm{C}\left\{{ }^{1} \mathrm{H}\right\}(\delta)$ \\
\hline 1 & $6.02(\mathrm{~d}, 4.1)$ & 73.8 & $6.02(\mathrm{~d}, 3.7)$ & 73.8 \\
\hline 2 & $5.78(\mathrm{dd}, 4.1,2.3)$ & 69.3 & $5.78(\mathrm{dd}, 3.7,2.3)$ & 69.2 \\
\hline 3 & $5.15(\mathrm{~d}, 2.3)$ & 76.5 & $5.15(\mathrm{~d}, 2.3)$ & 76.5 \\
\hline 4 & & 71.0 & & 71.0 \\
\hline 5 & & 94.6 & & 94.5 \\
\hline 6 & $7.19(\mathrm{~s})$ & 74.0 & $7.19(\mathrm{~s})$ & 73.9 \\
\hline 7 & $1.70(\mathrm{~d}, 3.5)$ & 50.5 & $1.70(\mathrm{~d}, 3.6)$ & 50.5 \\
\hline 8 & $5.64(\mathrm{dd}, 5.7,3.5)$ & 69.3 & $5.64(\mathrm{dd}, 6.0,3.6)$ & 69.2 \\
\hline 9 & $5.67(\mathrm{~d}, 5.7)$ & 70.9 & $5.67(\mathrm{~d}, 6.0)$ & 70.9 \\
\hline 10 & & 52.6 & & 52.6 \\
\hline 11 & & 84.1 & & 84.1 \\
\hline 12 & $1.31(\mathrm{~s})$ & 17.9 & $1.31(\mathrm{~s})$ & 17.9 \\
\hline $13 \mathrm{a}$ & $5.86(\mathrm{~d}, 11.5)$ & 69.7 & $5.87(\mathrm{~d}, 11.4)$ & 69.7 \\
\hline $13 b$ & $3.08(\mathrm{~d}, 11.5)$ & & $3.08(\mathrm{~d}, 11.4)$ & \\
\hline $14 \mathrm{a}$ & $5.46(\mathrm{~d}, 13.2)$ & 60.2 & $5.46(\mathrm{~d}, 13.2)$ & 60.2 \\
\hline $14 b$ & $4.77(\mathrm{~d}, 13.2)$ & & $4.77(\mathrm{~d}, 13.2)$ & \\
\hline 15 & $1.75(\mathrm{~s})$ & 23.4 & $1.75(\mathrm{~s})$ & 23.4 \\
\hline 2 ' & & 165.7 & & 165.6 \\
\hline $3^{\prime}$ & & 125.5 & & 125.5 \\
\hline $4^{\prime}$ & $7.55(\mathrm{dd}, 8.0,1.7)$ & 137.3 & $7.55(\mathrm{dd}, 7.8,1.8)$ & 137.3 \\
\hline $5^{\prime}$ & $6.52(\mathrm{dd}, 8.0,5.2)$ & 120.8 & $6.52(\mathrm{dd}, 7.8,4.6)$ & 120.8 \\
\hline $6^{\prime}$ & $8.33(\mathrm{dd}, 5.2,1.7)$ & 151.5 & $8.33(\mathrm{dd}, 4.6,1.8)$ & 151.5 \\
\hline $7^{\prime}$ & $4.99(\mathrm{q}, 7.5)$ & 37.1 & $4.99(\mathrm{q}, 7.4)$ & 37.1 \\
\hline $8^{\prime}$ & $2.72(\mathrm{q}, 7.5)$ & 45.2 & $2.72(\mathrm{q}, 7.3)$ & 45.2 \\
\hline $9^{\prime}$ & $1.57(\mathrm{~d}, 7.5)$ & 12.4 & $1.57(\mathrm{~d}, 7.4)$ & 12.3 \\
\hline $10^{\prime}$ & $1.28(\mathrm{~d}, 7.5)$ & 9.5 & $1.27(\mathrm{~d}, 7.3)$ & 9.5 \\
\hline
\end{tabular}


Supporting Information

\begin{tabular}{|c|c|c|c|c|}
\hline \multicolumn{2}{|l|}{$11^{\prime}$} & \multicolumn{2}{|l|}{174.1} & 174.1 \\
\hline \multicolumn{2}{|l|}{$12^{\prime}$} & \multicolumn{2}{|l|}{168.55} & 168.55 \\
\hline $\mathrm{OH}$ & $4.67(\mathrm{~s})$ & & $4.68(\mathrm{~s})$ & \\
\hline \multirow[t]{12}{*}{$\mathrm{Ac}$} & $2.23(\mathrm{~s})$ & 169.8 & $2.23(\mathrm{~s})$ & 169.8 \\
\hline & $1.91(\mathrm{~s})$ & 169.7 & $1.91(\mathrm{~s})$ & 169.7 \\
\hline & $1.88(\mathrm{~s})$ & 169.5 & $1.88(\mathrm{~s})$ & 169.5 \\
\hline & $1.67(\mathrm{~s})$ & 169.0 & $1.67(\mathrm{~s})$ & 169.0 \\
\hline & $1.66(\mathrm{~s})$ & 168.9 & $1.66(\mathrm{~s})$ & 168.9 \\
\hline & \multirow[t]{7}{*}{$1.61(\mathrm{~s})$} & 168.45 & $1.61(\mathrm{~s})$ & 168.46 \\
\hline & & 21.25 & & 21.2 \\
\hline & & 21.23 & & 21.2 \\
\hline & & 20.7 & & 20.7 \\
\hline & & 20.3 & & 20.3 \\
\hline & & 20.2 & & 20.2 \\
\hline & & 20.1 & & 20.1 \\
\hline
\end{tabular}

${ }^{a}$ Purchased from ALB Technology Limited. 


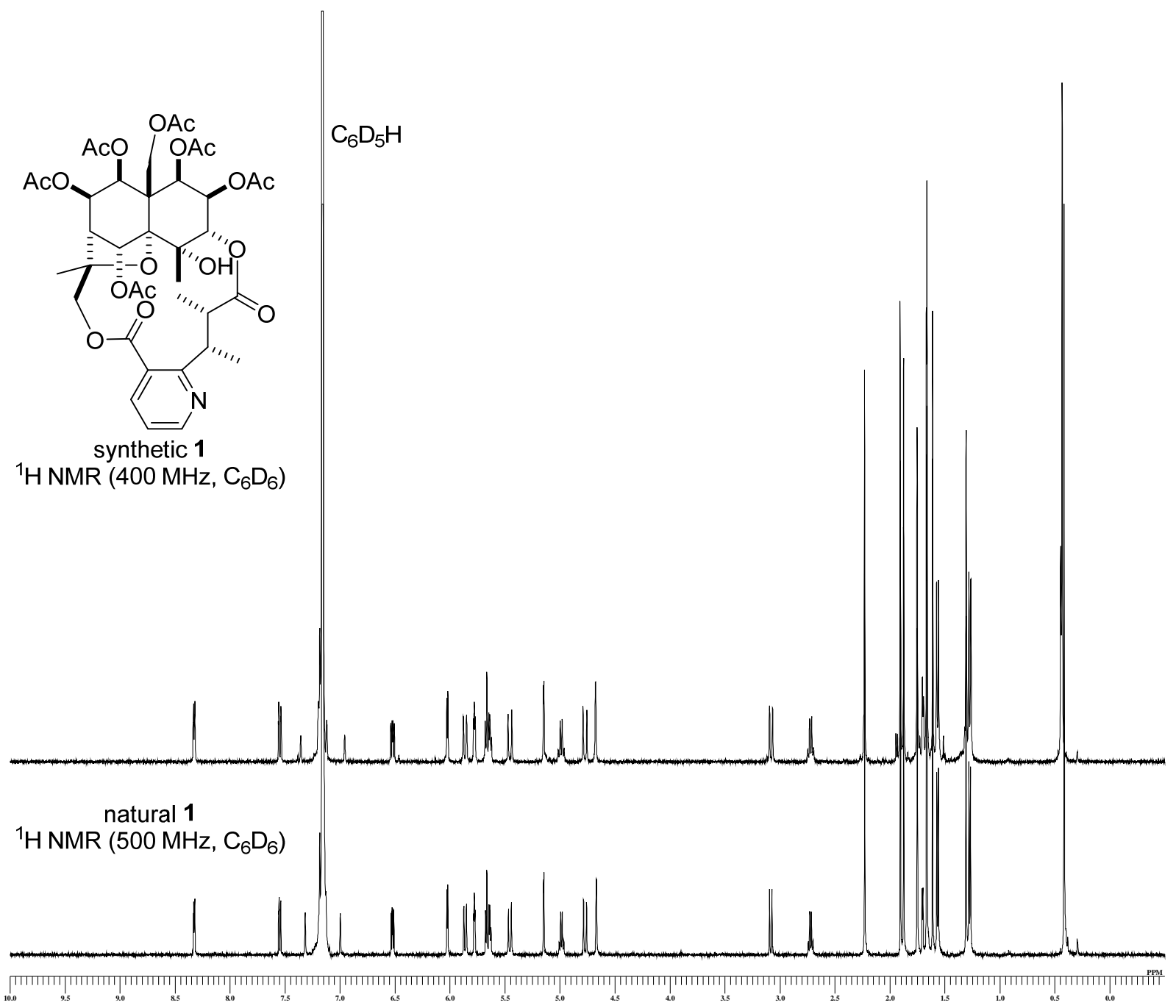

Figure S2. ${ }^{1}$ H NMR spectra of synthetic $1(400 \mathrm{MHz})$ and natural $1(500 \mathrm{MHz})$ 


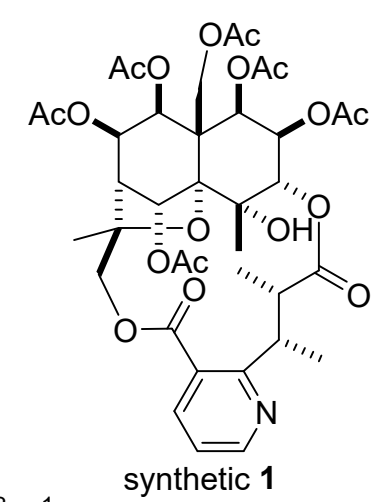

$\mathrm{C}_{6} \mathrm{D}_{6}$

${ }^{13} \mathrm{C}\left\{{ }^{1} \mathrm{H}\right\}$ NMR $\left(125 \mathrm{MHz}, \mathrm{C}_{6} \mathrm{D}_{6}\right)$

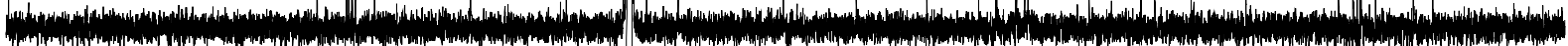
natural 1

${ }^{13} \mathrm{C}\left\{{ }^{1} \mathrm{H}\right\}$ NMR $\left(125 \mathrm{MHz}, \mathrm{C}_{6} \mathrm{D}_{6}\right)$

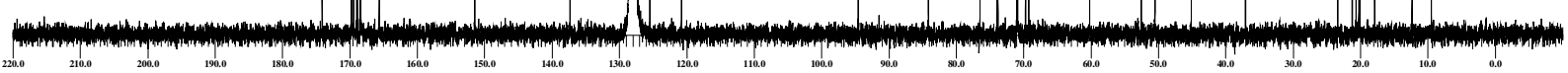

Figure S3. ${ }^{13} \mathrm{C}\left\{{ }^{1} \mathrm{H}\right\}$ NMR spectra of synthetic $1(125 \mathrm{MHz})$ and natural $1(125 \mathrm{MHz})$ 


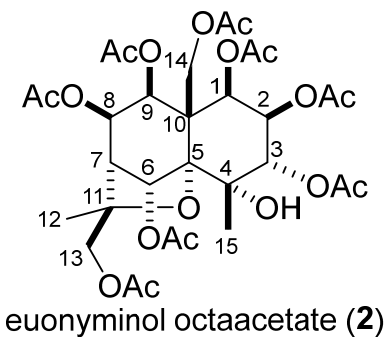

Table S3. ${ }^{1} \mathrm{H}$ and ${ }^{13} \mathrm{C}\left\{{ }^{1} \mathrm{H}\right\}$ NMR data of natural, ${ }^{\mathrm{S} 3}$ reported ${ }^{\mathrm{S} 4}$ and synthetic euonyminol octaacetate (2) in $\mathrm{CDCl}_{3}$.

\begin{tabular}{|c|c|c|c|c|c|c|}
\hline & \multicolumn{2}{|c|}{ natural $2(300-500,75-125 \mathrm{MHz})^{\mathrm{S3}}$} & \multicolumn{2}{|c|}{ reported $2(500,125 \mathrm{MHz})^{\mathrm{S4}}$} & \multicolumn{2}{|c|}{ synthetic $2(400,125 \mathrm{MHz})$} \\
\hline No. & $\mathrm{H}[\delta$, multi, $J(\mathrm{~Hz})]$ & ${ }^{13} \mathrm{C}\left\{{ }^{1} \mathrm{H}\right\}(\delta)$ & ${ }^{1} \mathrm{H}[\delta$, multi, $J(\mathrm{~Hz})]$ & ${ }^{13} \mathrm{C}\left\{{ }^{1} \mathrm{H}\right\}(\delta)$ & ${ }^{1} \mathrm{H}[\delta$, multi, $J(\mathrm{~Hz})]$ & ${ }^{13} \mathrm{C}\left\{{ }^{1} \mathrm{H}\right\}(\delta)$ \\
\hline 1 & $5.58(\mathrm{~d}, 3.6)$ & 73.1 & $5.58(\mathrm{~d}, 3.7)$ & 73.2 & $5.58(\mathrm{~d}, 3.7)$ & 73.1 \\
\hline 2 & $5.26(\mathrm{dd}, 3.6,2.8)$ & 69.0 & $5.27(\mathrm{~m})$ & 69.0 & $5.27(\mathrm{dd}, 3.7,2.8)$ & 69.0 \\
\hline 3 & $4.82(\mathrm{~d}, 2.8)$ & 75.8 & $4.82(\mathrm{~d}, 2.5)$ & 75.8 & $4.82(\mathrm{~d}, 2.8)$ & 75.8 \\
\hline 4 & & 69.3 & & 69.3 & & 69.3 \\
\hline 5 & & 92.9 & & 93.0 & & 92.9 \\
\hline 6 & $6.76(\mathrm{~s})$ & 73.9 & $6.77(\mathrm{~s})$ & 73.9 & $6.77(\mathrm{~s})$ & 73.9 \\
\hline 7 & $2.32(\mathrm{~d}, 3.6)$ & 50.7 & $2.32(\mathrm{~d}, 3.9)$ & 50.7 & $2.32(\mathrm{~d}, 3.7)$ & 50.7 \\
\hline 8 & $5.48(\mathrm{dd}, 5.9,3.6)$ & 69.0 & $5.48(\mathrm{dd}, 5.8,3.9)$ & 69.0 & $5.48(\mathrm{dd}, 6.0,3.7)$ & 68.9 \\
\hline 9 & $5.34(\mathrm{~d}, 5.9)$ & 71.0 & $5.34(\mathrm{~d}, 6.0)$ & 70.9 & $5.34(\mathrm{~d}, 6.0)$ & 70.9 \\
\hline 10 & & 51.9 & & 51.9 & & 51.9 \\
\hline 11 & & 84.1 & & 84.1 & & 84.1 \\
\hline 12 & $1.56(\mathrm{~s})$ & 18.3 & $1.57(\mathrm{~s})$ & 18.3 & $1.57(\mathrm{~s})$ & 18.3 \\
\hline $13 \mathrm{a}$ & $4.89(\mathrm{~d}, 11.6)$ & 69.4 & $4.89(\mathrm{~d}, 11.6)$ & 69.5 & $4.90(\mathrm{~d}, 11.9)$ & 69.4 \\
\hline $13 b$ & $3.94(\mathrm{~d}, 11.6)$ & & $3.95(\mathrm{~d}, 11.6)$ & & $3.93(\mathrm{~d}, 11.9)$ & \\
\hline $14 \mathrm{a}$ & $5.25(\mathrm{~d}, 13.3)^{a}$ & 60.3 & $5.21(\mathrm{~d}, 13.3)$ & 60.3 & $5.20(\mathrm{~d}, 13.3)$ & 60.3 \\
\hline $14 \mathrm{~b}$ & $4.42(\mathrm{~d}, 13.3)$ & & $4.42(\mathrm{~d}, 13.3)$ & & $4.42(\mathrm{~d}, 13.3)$ & \\
\hline 15 & $1.47(\mathrm{~s})$ & 23.2 & $1.48(\mathrm{~d}, 1.4)$ & 23.3 & $1.47(\mathrm{~d}, 1.4)$ & 23.2 \\
\hline $\mathrm{OH}$ & & & & & $4.20(\mathrm{~d}, 1.4)$ & \\
\hline Ac-1 & & 169.3 & & 169.3 & & 169.3 \\
\hline & $1.88(\mathrm{~s})$ & 20.5 & $1.88(\mathrm{~s})$ & 20.5 & $1.88(\mathrm{~s})$ & 20.5 \\
\hline Ac-2 & & 168.7 & & 168.7 & & 168.7 \\
\hline & $2.12(\mathrm{~s})$ & 20.8 & $2.12(\mathrm{~s})$ & 20.8 & $2.12(\mathrm{~s})$ & 20.8 \\
\hline Ac-3 & & 169.5 & & 169.5 & & 169.6 \\
\hline & $2.15(\mathrm{~s})$ & 20.8 & $2.16(\mathrm{~s})$ & 20.9 & $2.16(\mathrm{~s})$ & 20.9 \\
\hline Ac- 6 & & 169.8 & & 169.8 & & 169.8 \\
\hline & $2.13(\mathrm{~s})$ & 21.0 & $2.13(\mathrm{~s})$ & 21.0 & $2.13(\mathrm{~s})$ & 21.0 \\
\hline Ac- 8 & & 170.0 & & 170.1 & & 170.1 \\
\hline & $2.16(\mathrm{~s})$ & 21.0 & $2.17(\mathrm{~s})$ & 21.0 & $2.16(\mathrm{~s})$ & 21.0 \\
\hline
\end{tabular}




\begin{tabular}{llrlrrr} 
Ac-9 & & 168.9 & & 168.9 & 169.0 \\
& $1.98(\mathrm{~s})$ & 20.5 & $1.98(\mathrm{~s})$ & 20.5 & $1.98(\mathrm{~s})$ & 20.5 \\
Ac-13 & & 170.7 & & 170.7 & & 170.8 \\
& $2.11(\mathrm{~s})$ & 21.4 & $2.11(\mathrm{~s})$ & 21.5 & $2.11(\mathrm{~s})$ & 21.5 \\
\multirow{2}{*}{ Ac-14 } & & 170.1 & & 170.1 & & 170.1 \\
& $2.25(\mathrm{~s})$ & 21.2 & $2.25(\mathrm{~s})$ & 21.3 & $2.25(\mathrm{~s})$ & 21.2 \\
\hline
\end{tabular}

${ }^{a}$ The $\delta$ value of this peak should be misassigned. 


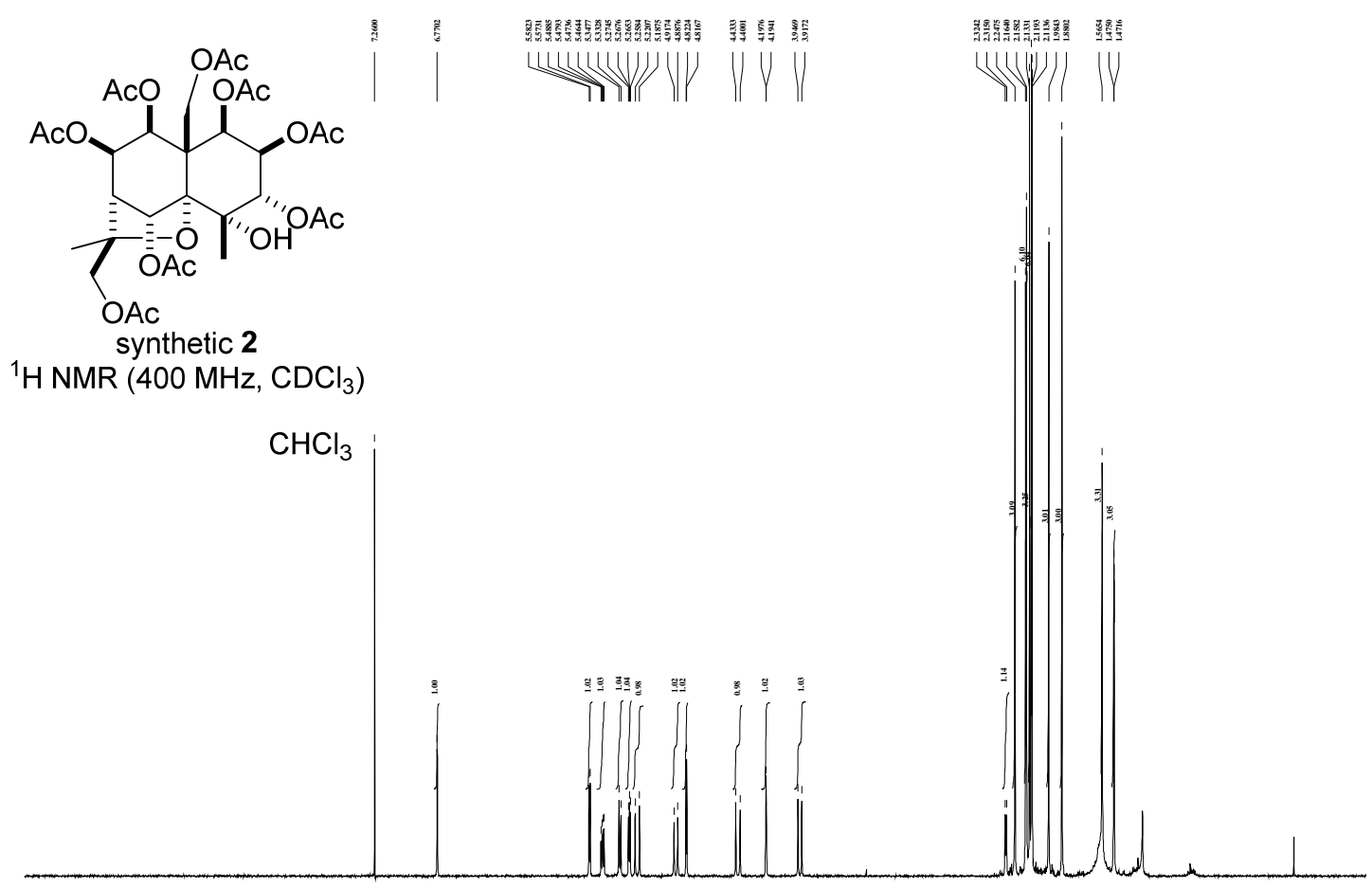

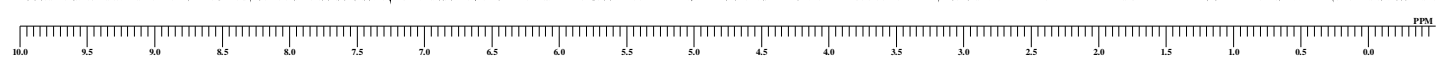

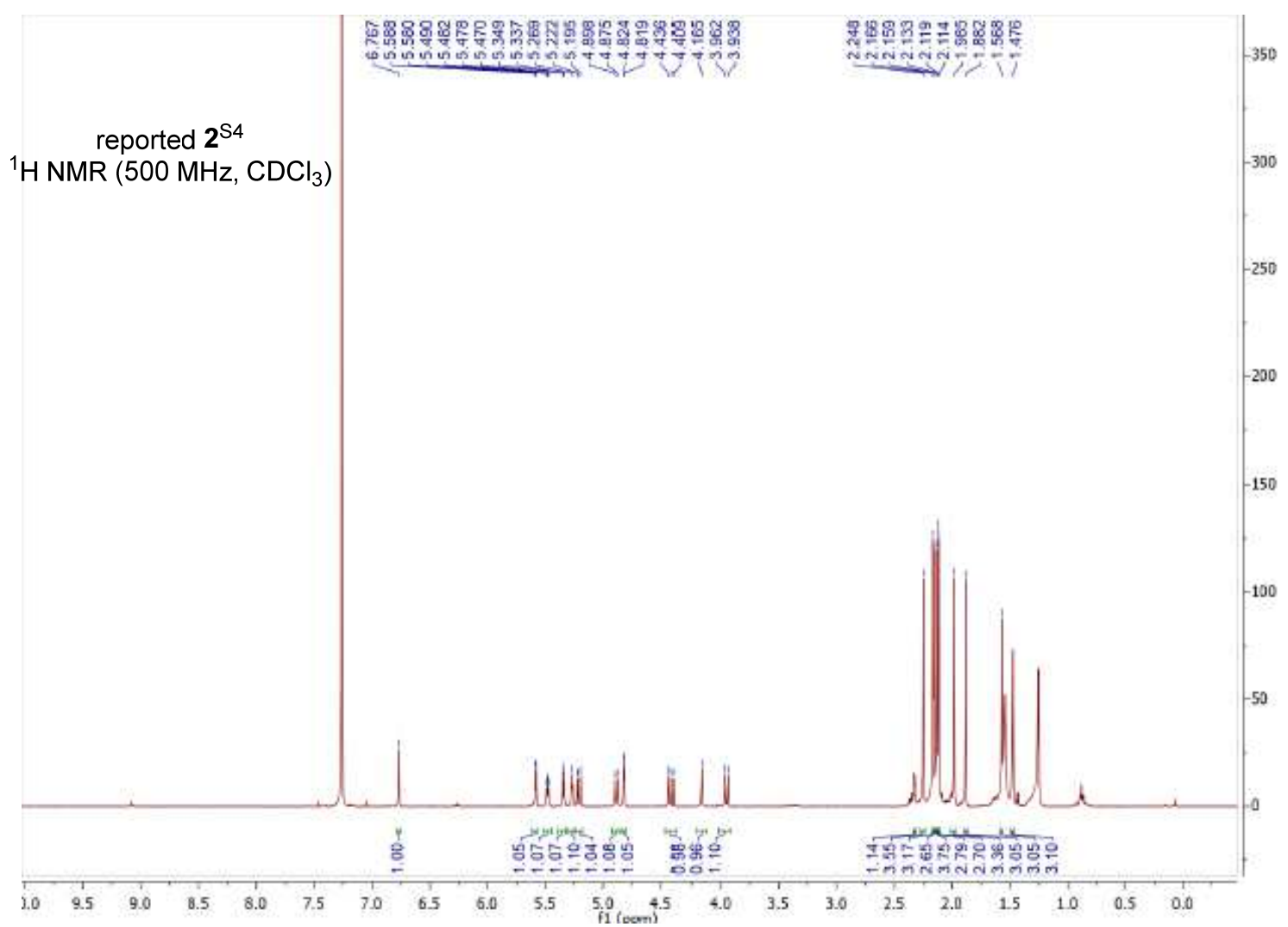

Figure S4. ${ }^{1} \mathrm{H}$ NMR spectra of synthetic $2(400 \mathrm{MHz})$ and reported $2(500 \mathrm{MHz})$ 


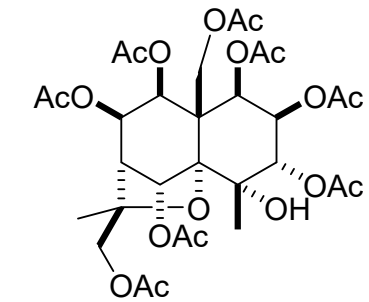

synthetic 2

${ }^{13} \mathrm{C}\left\{{ }^{1} \mathrm{H}\right\}$ NMR $\left(125 \mathrm{MHz}, \mathrm{CDCl}_{3}\right)$
$\mathrm{CDCl}_{3}$

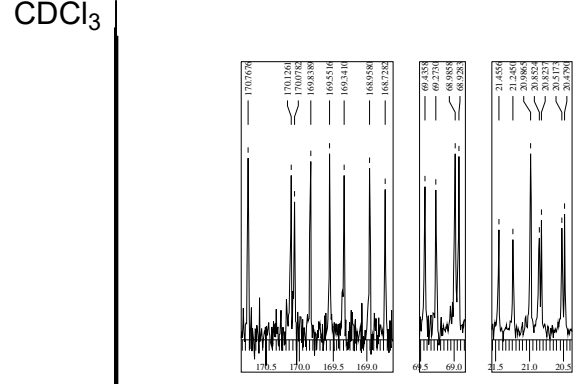

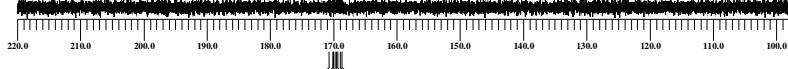

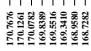

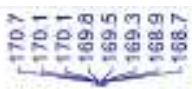

reported $2^{\mathrm{S} 4}$

${ }^{13} \mathrm{C}\left\{{ }^{1} \mathrm{H}\right\}$ NMR $\left(125 \mathrm{MHz}, \mathrm{CDCl}_{3}\right)$

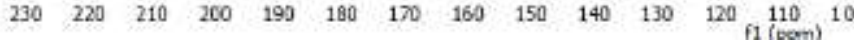

Figure S5. ${ }^{13} \mathrm{C}\left\{{ }^{1} \mathrm{H}\right\}$ NMR spectra of synthetic $2(125 \mathrm{MHz})$ and reported $2(125 \mathrm{MHz})$ 


\section{X-ray structures and crystallographic data of 56 and 63}

Table S4. Crystal data and structure refinement for $\mathbf{5 6}$ and 63

\begin{tabular}{|c|c|c|}
\hline Compound & 56 & 63 \\
\hline CCDC number & 2120480 & 2115563 \\
\hline Molecular formula & $\mathrm{C}_{49} \mathrm{H}_{85} \mathrm{NO}_{13} \mathrm{Si}_{3}$ & $\mathrm{C}_{49} \mathrm{H}_{85} \mathrm{NO}_{13} \mathrm{Si}_{3}$ \\
\hline Formula weight & 980.44 & 980.44 \\
\hline Temperature (K) & 93 & 100 \\
\hline Wavelength $(\AA)$ & 0.71073 & 0.71073 \\
\hline Crystal color, habit & colorless, plate & colorless, block \\
\hline Crystal size $\left(\mathrm{mm}^{3}\right)$ & $0.30 \times 0.20 \times 0.05$ & $0.20 \times 0.20 \times 0.20$ \\
\hline Crystal system & orthorhombic & orthorhombic \\
\hline Space group & $\mathrm{P} 2{ }_{1} 2_{1} 2_{1}(\# 19)$ & $\mathrm{P} 2{ }_{1} 2_{1} 2_{1}(\# 19)$ \\
\hline \multicolumn{3}{|l|}{ Unit cell dimensions } \\
\hline a $(\AA)$ & $12.0988(4)$ & $14.2621(5)$ \\
\hline $\mathrm{b}(\AA)$ & 19.1871(5) & $15.8388(6)$ \\
\hline c $(\AA)$ & $23.6915(7)$ & $24.7859(9)$ \\
\hline$\alpha\left(^{\circ}\right)$ & 90 & 90 \\
\hline$\beta\left({ }^{\circ}\right)$ & 90 & 90 \\
\hline$\gamma\left({ }^{\circ}\right)$ & 90 & 90 \\
\hline Volume $\left(\AA^{3}\right)$ & $5499.8(3)$ & $5599.0(4)$ \\
\hline$Z$ & 4 & 4 \\
\hline Density (calculated) $\left(\mathrm{g} / \mathrm{cm}^{3}\right)$ & 1.184 & 1.163 \\
\hline$\mu(\operatorname{MoK} \alpha)\left(\mathrm{cm}^{-1}\right)$ & 1.45 & 1.42 \\
\hline $\mathrm{F}(000)$ & 2128 & 2128 \\
\hline \multirow[t]{3}{*}{ Index ranges } & $-16<=\mathrm{h}<15$ & $-19<=\mathrm{h}<16$ \\
\hline & $-25<=\mathrm{k}<23$ & $-21<=\mathrm{k}<20$ \\
\hline & $-31<=1<29$ & $-33<=1<33$ \\
\hline Reflections collected & 43716 & 79743 \\
\hline Independent reflections & 12413 & 13481 \\
\hline $\mathrm{R}$ (int) & 0.1672 & 0.0500 \\
\hline Completeness to theta & $25.242^{\circ}, 99.7 \%$ & $25.242^{\circ}, 99.9 \%$ \\
\hline Max. and min. transmission & $1.000,0.42035$ & $1.000,0.67191$ \\
\hline Refinement method & $\begin{array}{l}\text { Full-matrix least- } \\
\text { squares on } \mathrm{F}^{2}\end{array}$ & $\begin{array}{l}\text { Full-matrix least- } \\
\text { squares on } \mathrm{F}^{2}\end{array}$ \\
\hline $\begin{array}{l}\text { No. Observations } \\
\text { (All reflections) }\end{array}$ & 12413 & 13481 \\
\hline No. Variables & 618 & 813 \\
\hline Reflection/Parameter Ratio & 20.09 & 16.58 \\
\hline Goodness-of-fit on $\mathrm{F}^{2}$ & 0.996 & 1.058 \\
\hline Residuals: R1 $(I>2.00 \sigma(I))$ & 0.0556 & 0.0656 \\
\hline $\begin{array}{l}\text { Residuals: } \mathrm{R} \\
\text { (All reflections) }\end{array}$ & 0.0660 & 0.0865 \\
\hline $\begin{array}{l}\text { Residuals: wR2 } \\
\text { (All reflections) }\end{array}$ & 0.1325 & 0.1304 \\
\hline $\begin{array}{l}\text { Max. and min. peak in Final } \\
\text { Diff. Map }\left(\mathrm{e}-/ \AA^{3}\right)\end{array}$ & $0.54,-0.44$ & $0.83,-1.0$ \\
\hline
\end{tabular}




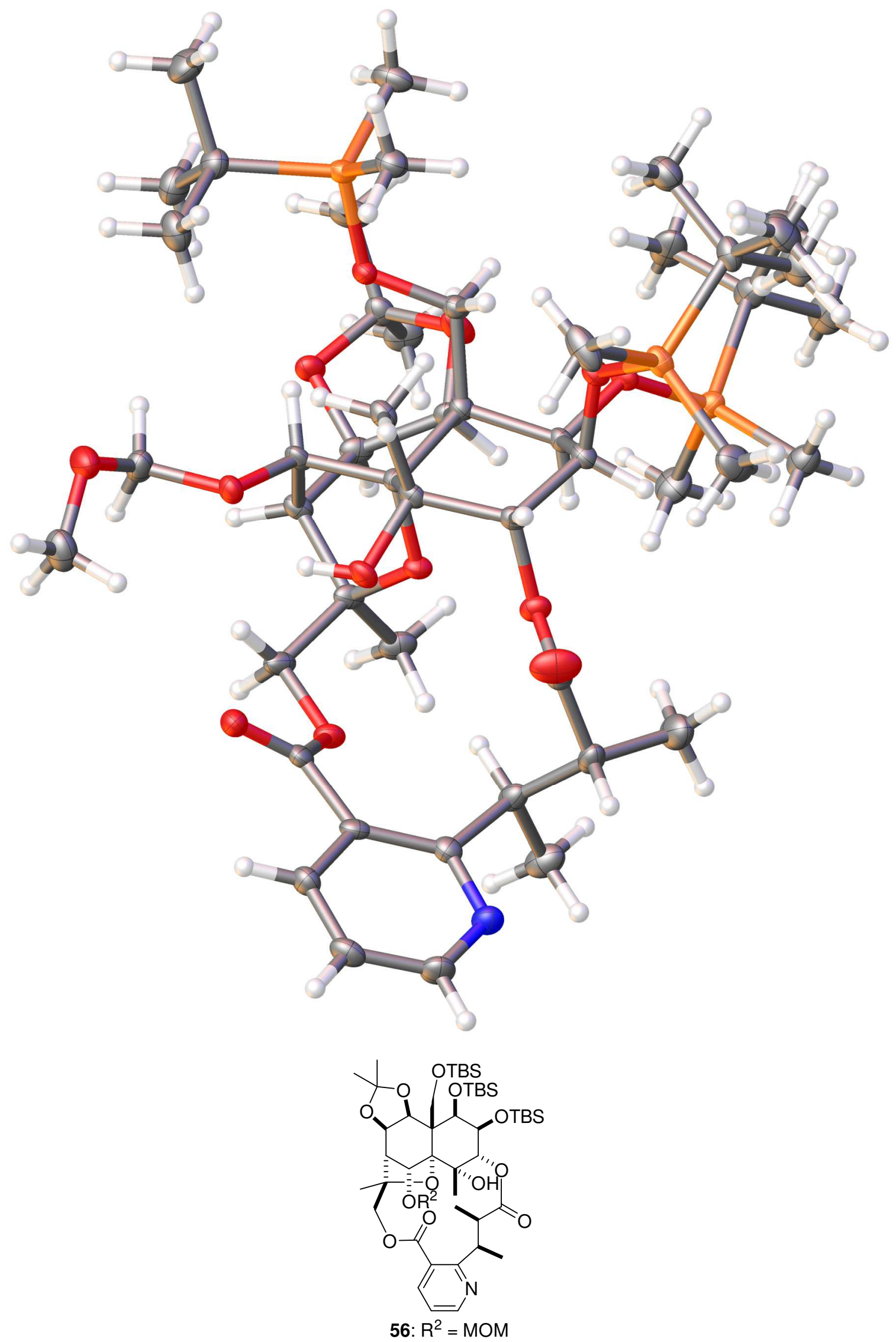

Figure S6. X-ray crystallographic structure of 56 (CCDC 2120480) with thermal ellipsoids at the $50 \%$ probability level (ORTEP) 


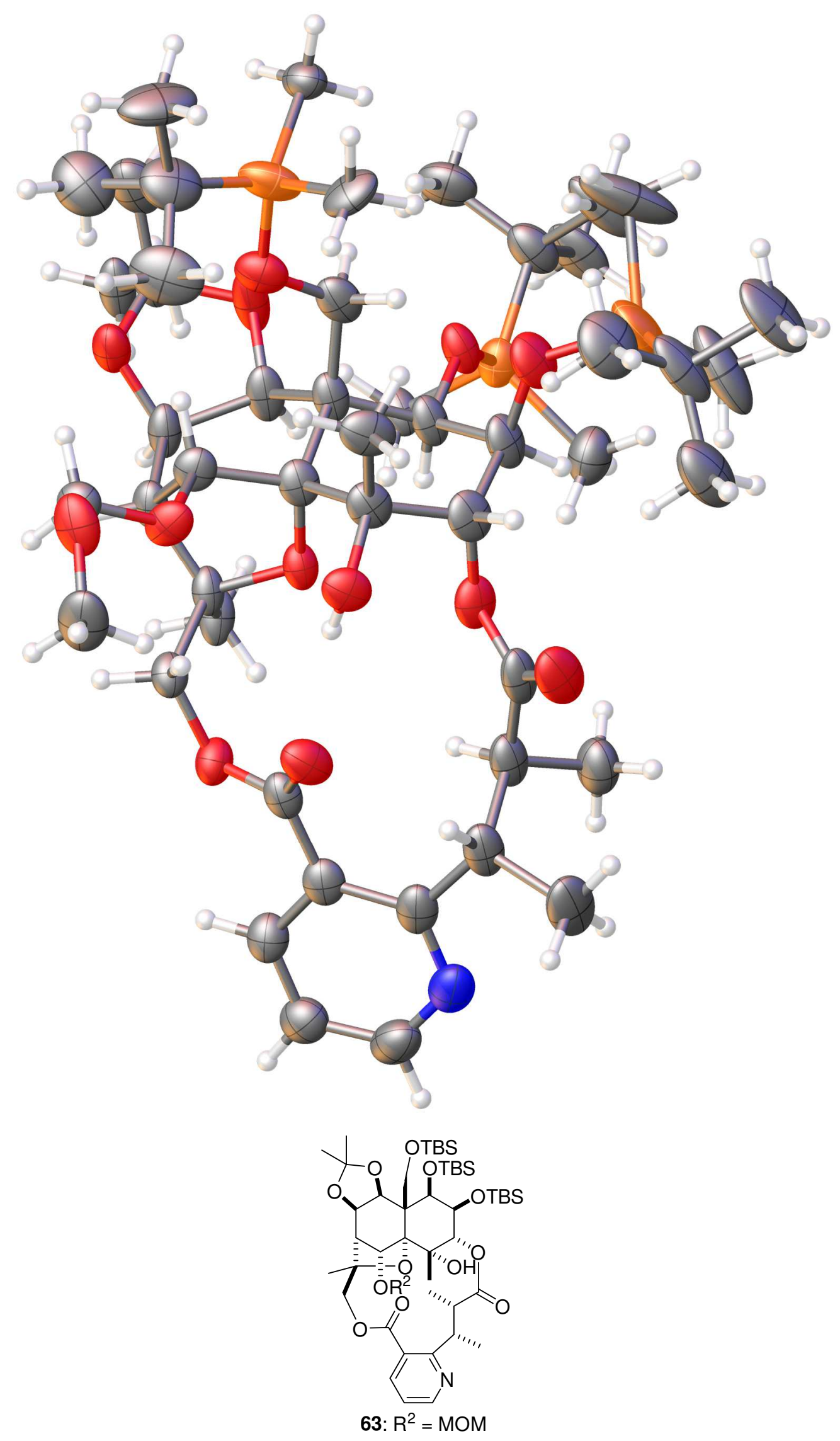

Figure S7. X-ray crystallographic structure of 63 (CCDC 2115563) with thermal ellipsoids at the $50 \%$ probability level (ORTEP) 


\section{Determination of enantiopurity of alcohol 13 and enone 18}

\section{4-1. Determination of enantiopurity of alcohol 13}

Conditions: CHIRALCEL OD-H (Daicel), hexane $/ i$-PrOH =98/2, $1.0 \mathrm{~mL} / \mathrm{min}, 220 \mathrm{~nm}$,<smiles>C=C(C(=O)OCC)[C@H](O)C1COC(C)(C)O1</smiles>

13

(enantio-enriched product)

$t_{R}(\min )=8.42$, peak area $(\%)=8.837$

$t_{R}(\min )=9.39$, peak area $(\%)=91.163$

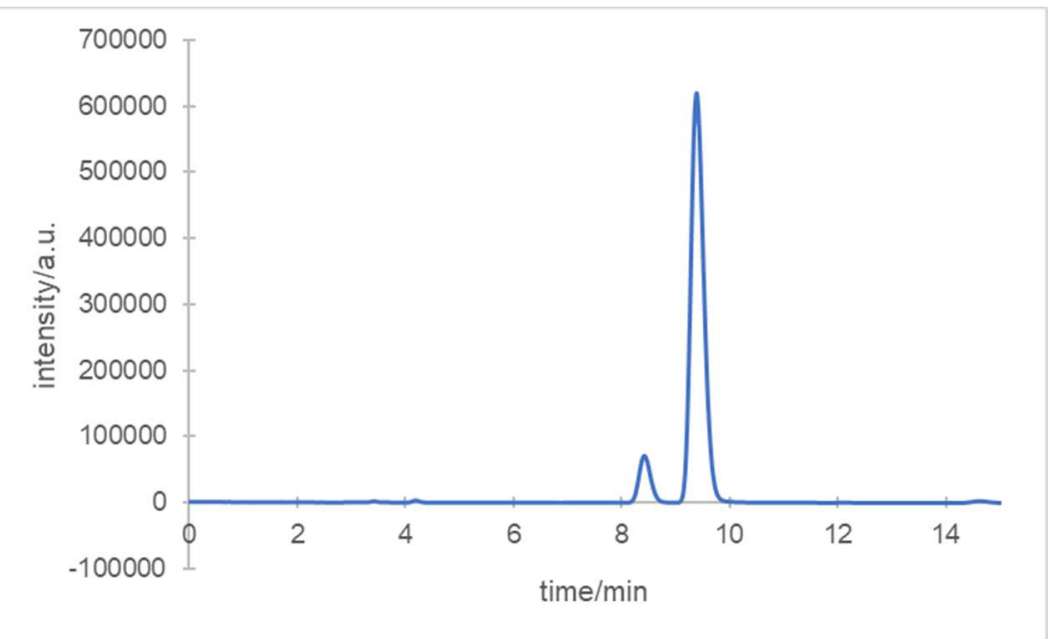




\section{4-2. Determination of enantiopurity of enone 18}

Conditions: CHIRALPAK AS-RH (Daicel), $\mathrm{MeCN} / \mathrm{H}_{2} \mathrm{O}=45 / 55,1.0 \mathrm{~mL} / \mathrm{min}, 254 \mathrm{~nm}$

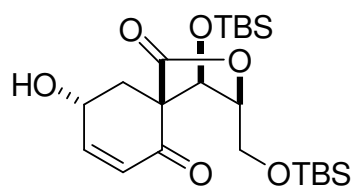

18

(enantio-enriched product)

$t_{R}(\min )=17.07$, peak area $(\%)=90.743$

$t_{R}(\min )=20.86$, peak area $(\%)=9.257$

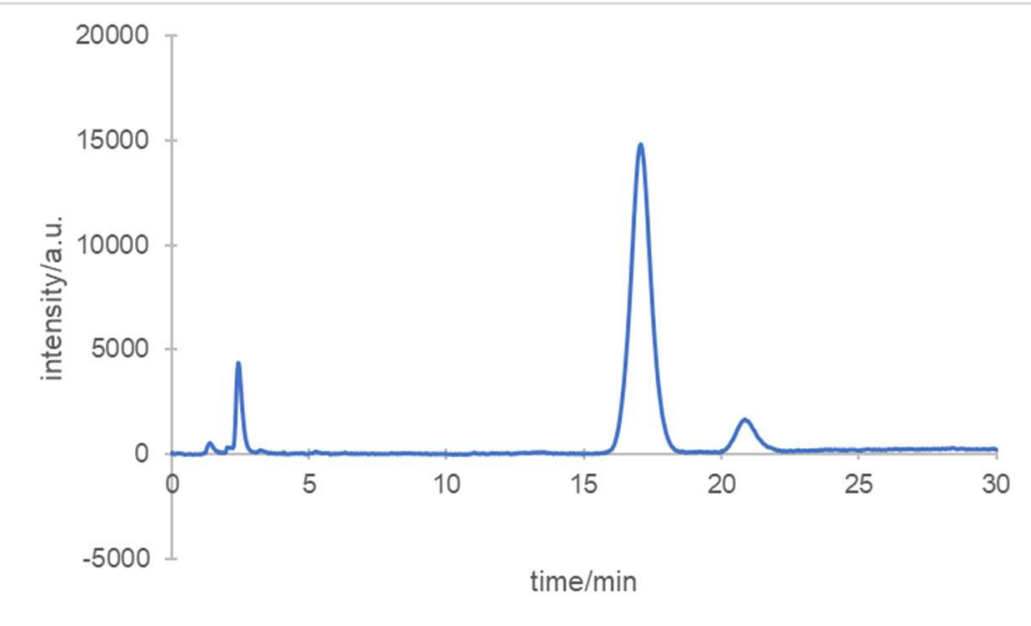

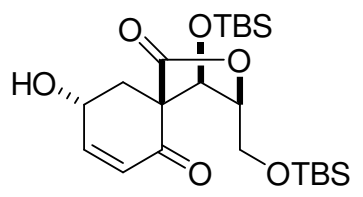

18

(enantiopure product recrystallized from toluene)

$t_{\mathrm{R}}(\min )=17.75$, peak area $(\%)=99.997$ $t_{R}(\min )=21.93$, peak area $(\%)=0.003$

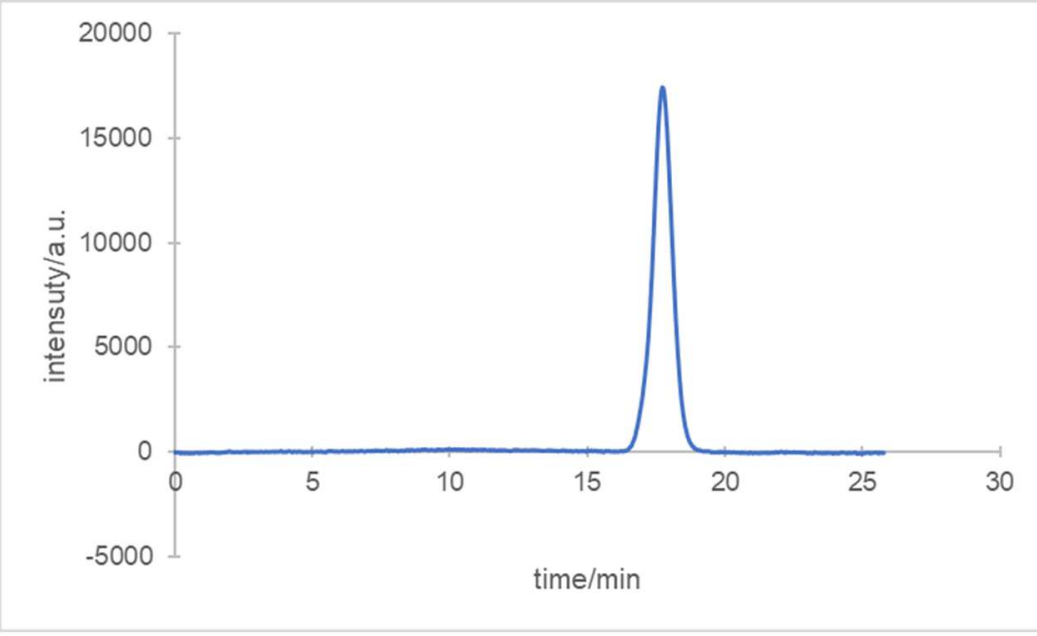




\section{Structural assignment of Diels-Alder adducts 15}

The structure of 15-exo was assigned by the derivatization into tricycle $\mathbf{S 1 0}$. The C10-stereochemistry of 15-exo was confirmed by the NOE experiment.

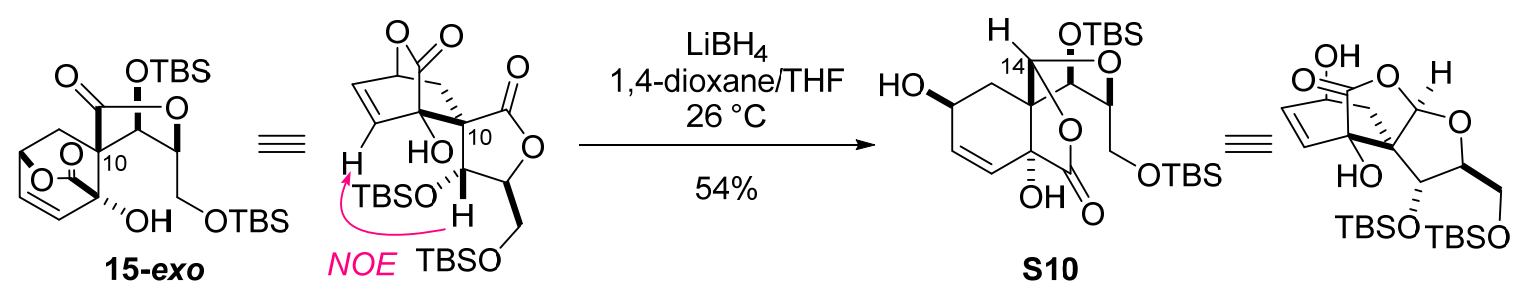

Tricycle S10. $\mathrm{LiBH}_{4}(2.0 \mathrm{M}$ THF solution, $17.5 \mu \mathrm{L}, 35.0 \mu \mathrm{mol})$ was added to a solution of DielsAlder adduct 15-exo $(14.0 \mathrm{mg}, 28.9 \mu \mathrm{mol})$ in 1,4-dioxane $(140 \mu \mathrm{L})$ and THF $(140 \mu \mathrm{L})$ at $26^{\circ} \mathrm{C}$. After the reaction mixture was stirred at $26^{\circ} \mathrm{C}$ for $45 \mathrm{~min}$, saturated aqueous $\mathrm{NH}_{4} \mathrm{Cl}(1.5 \mathrm{~mL})$ was added to the mixture at $0{ }^{\circ} \mathrm{C}$. The resultant mixture was extracted with EtOAc $(1.5 \mathrm{~mL} \mathrm{x} 3)$. The combined organic layers were dried over $\mathrm{Na}_{2} \mathrm{SO}_{4}$, filtered, and concentrated. The residue was purified by flash column chromatography on silica gel $(1 \mathrm{~g}$, hexane/EtOAc $=5 / 1$ to $3 / 1)$ to afford tricycle $\mathbf{S 1 0}(7.58 \mathrm{mg}$, $15.6 \mu \mathrm{mol})$ in $54 \%$ yield: white solid. m.p. $202-205^{\circ} \mathrm{C} .[\alpha]_{\mathrm{D}}{ }^{24}-58.2\left(c 0.379, \mathrm{CHCl}_{3}\right)$. IR (film): 3400 , 3325, 2952, 2929, 2856, 1757, 1252, 1165, 933, $837 \mathrm{~cm}^{-1} .{ }^{1} \mathrm{H}$ NMR (500 MHz, CDCl 3$): \delta 6.30(1 \mathrm{H}, \mathrm{s}$, H14), 6.20 (1H, dd, $J=9.8,5.2 \mathrm{~Hz}, \mathrm{H} 7), 5.74(1 \mathrm{H}, \mathrm{d}, J=9.8 \mathrm{~Hz}, \mathrm{H} 6), 4.65$ (1H, d, $J=7.5 \mathrm{~Hz}, \mathrm{H} 1)$, $4.50(1 \mathrm{H}, \mathrm{dd}, J=5.2,5.2 \mathrm{~Hz}, \mathrm{H} 8), 4.08$ (1H, ddd, $J=7.5,4.0,2.3 \mathrm{~Hz}, \mathrm{H} 2), 3.88$ (1H, dd, $J=12.0,2.3$ $\mathrm{Hz}, \mathrm{H} 3 \mathrm{a}), 3.71(1 \mathrm{H}, \mathrm{dd}, J=12.0,4.0 \mathrm{~Hz}, \mathrm{H} 3 \mathrm{~b}), 2.91(1 \mathrm{H}$, br s, OH), $2.09(1 \mathrm{H}, \mathrm{dd}, J=16.0,5.2 \mathrm{~Hz}$, H9a), $1.99(1 \mathrm{H}, \mathrm{d}, J=16.0 \mathrm{~Hz}, \mathrm{H} 9 \mathrm{~b}), 1.85(1 \mathrm{H}, \mathrm{br} \mathrm{s}, \mathrm{OH}), 0.91(9 \mathrm{H}, \mathrm{s}, t$-Bu of TBS), $0.86(9 \mathrm{H}, \mathrm{s}, t$ Bu of TBS), 0.09 (3H, s, CH3 of TBS), $0.08\left(9 \mathrm{H}, \mathrm{s}, \mathrm{CH} 3\right.$ of TBS x3). ${ }^{13} \mathrm{C}\left\{{ }^{1} \mathrm{H}\right\} \mathrm{NMR}\left(125 \mathrm{MHz}, \mathrm{CDCl}_{3}\right)$ : $\delta 175.6,132.7,127.3,108.0,88.1,74.6,71.2,62.02,61.97,54.5,26.2,25.9$ (3C), 25.8 (3C), 18.4, 18.1, $-4.7,-5.1,-5.3,-5.6$. HRMS (ESI-TOF) $[\mathrm{M}+\mathrm{Na}]^{+} \mathrm{m} / \mathrm{z}$ : Calcd for $\mathrm{C}_{23} \mathrm{H}_{42} \mathrm{O}_{7} \mathrm{Si}_{2} \mathrm{Na}$ 509.2361; Found 509.2356 . 
The structure of 15-endo was determined based on the result of the following experiment.
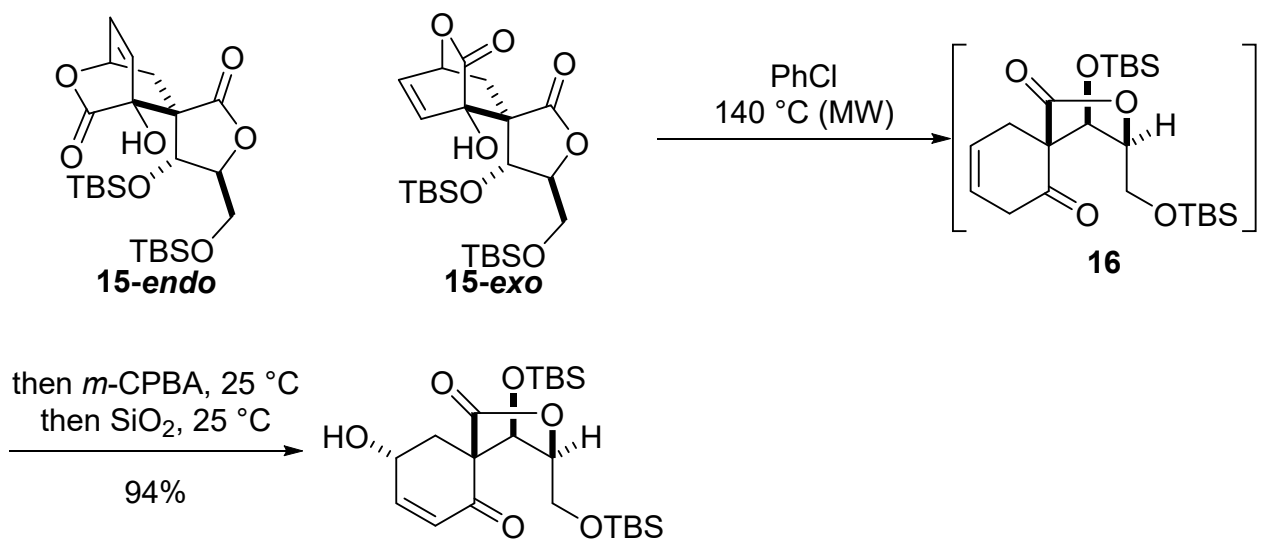

18

Enone 18 from Diels-Alder adducts 15-exo and 15-endo. A Pyrex vessel was charged with a 2.5:1 mixture of 15-exo and 15-endo (5.02 g, $10.4 \mathrm{mmol})$ and $\mathrm{PhCl}(15.0 \mathrm{~mL})$. The reaction mixture was heated to $140{ }^{\circ} \mathrm{C}$ under microwave irradiation for $1 \mathrm{~h}$. After the reaction mixture was cooled to room temperature, $m$-CPBA ( $77 \%$ purity, $3.50 \mathrm{~g}, 15.6 \mathrm{mmol}$ ) was added to the mixture at $25^{\circ} \mathrm{C}$. After being stirred at $25^{\circ} \mathrm{C}$ for $16 \mathrm{~h}$, the reaction mixture was poured into a $1: 1$ mixture of saturated $\mathrm{NaHCO}_{3}(50$ $\mathrm{mL})$ and saturated aqueous $\mathrm{Na}_{2} \mathrm{~S}_{2} \mathrm{O}_{3}(100 \mathrm{~mL})$ at $0{ }^{\circ} \mathrm{C}$. The resultant mixture was extracted with $\mathrm{CH}_{2} \mathrm{Cl}_{2}$ (100 mL x3). The combined organic layers were dried over $\mathrm{Na}_{2} \mathrm{SO}_{4}$, filtered, and concentrated. The residue was treated with silica gel $(75 \mathrm{~g})$ at room temperature for $13 \mathrm{~h}$ and eluted with hexane/EtOAc (10/1 to 0/1) to afford enone 18 (4.48 g, $9.81 \mathrm{mmol})$ in 94\% yield. The result indicated that 15-exo and 15-endo were converted into the same compound $\mathbf{1 8 .}$ 


\section{Determination of the C8-configuration of enone 18}

The C8-configuration of enone 18 was determined to be $R$ by the modified Mosher mehod. ${ }^{\mathrm{S} 5}(S)$ - and $(R)$-MTPA esters S11a and S11b were synthesized by treatment of 18 with $(R)$ - and $(S)$-MTPA-Cl, respectively.

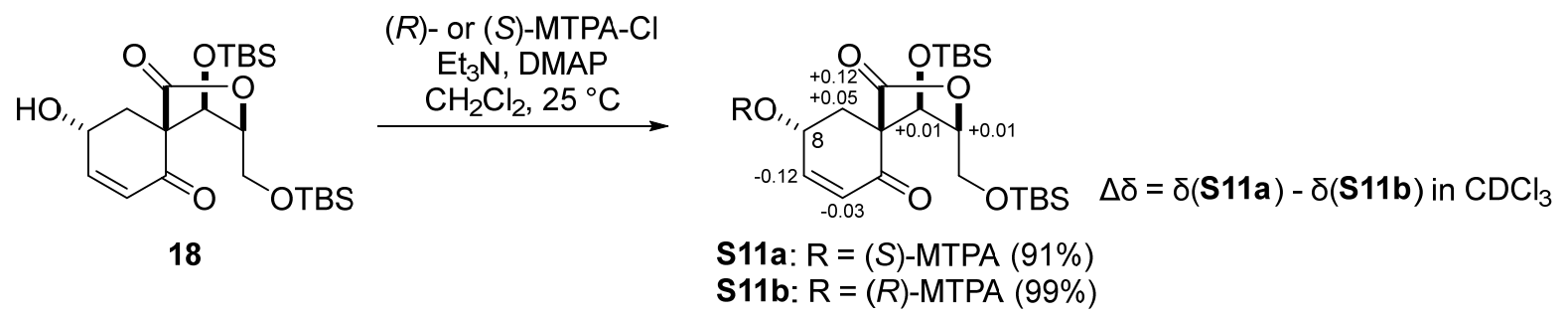

(S)-MTPA ester S11a. (R)-(-)- $\alpha$-Methoxy- $\alpha$-(trifluoromethyl)phenylacetyl chloride $(14.0 \mu \mathrm{L}, 74.8$ $\mu \mathrm{mol})$ and DMAP $(0.6 \mathrm{mg}, 5 \mu \mathrm{mol})$ were successively added to a solution of enone $\mathbf{1 8}(17.2 \mathrm{mg}, 37.7$ $\mu \mathrm{mol})$ and $\mathrm{Et}_{3} \mathrm{~N}(21.0 \mu \mathrm{L}, 152 \mu \mathrm{mol})$ in $\mathrm{CH}_{2} \mathrm{Cl}_{2}(380 \mu \mathrm{L})$ at $25^{\circ} \mathrm{C}$. After being stirred at $25^{\circ} \mathrm{C}$ for 30 min, the reaction mixture was diluted with toluene $(3 \mathrm{~mL})$ and concentrated. The residue was purified by flash column chromatography on silica gel $(0.5 \mathrm{~g}$, hexane/EtOAc $=5 / 1)$ to afford $(S)$-MTPA ester S11a $(23.1 \mathrm{mg}, 34.3 \mu \mathrm{mol})$ in $91 \%$ yield: white solid. m.p. $125-128{ }^{\circ} \mathrm{C}$. $[\alpha]_{\mathrm{D}}{ }^{24}-17.2\left(c 1.16, \mathrm{CHCl}_{3}\right)$. IR (film): 2932, 2858, 1774, 1688, 1470, 1254, 1185, 1122, 1055, $1017 \mathrm{~cm}^{-1} .{ }^{1} \mathrm{H}$ NMR (400 MHz, $\left.\mathrm{CDCl}_{3}\right): \delta$ 7.53-7.51 (2H, m, aromatic), 7.45-7.39 (3H, m, aromatic), $6.86(1 \mathrm{H}, \mathrm{ddd}, J=10.5,2.3,1.8$ $\mathrm{Hz}, \mathrm{H} 7), 6.22$ (1H, dd, $J=10.5,2.3 \mathrm{~Hz}, \mathrm{H6}), 6.17$ (1H, dddd, $J=11.0,5,5,2.3,2.3 \mathrm{~Hz}, \mathrm{H} 8), 5.42(1 \mathrm{H}$, $\mathrm{d}, J=7.8 \mathrm{~Hz}, \mathrm{H} 1), 4.20$ (1H, ddd, $J=7.8,3.2,2.3 \mathrm{~Hz}, \mathrm{H} 2), 4.02(1 \mathrm{H}, \mathrm{dd}, J=12.4,2.3 \mathrm{~Hz}, \mathrm{H} 3 \mathrm{a}), 3.80$ $(1 \mathrm{H}, \mathrm{dd}, J=12.4,3.2 \mathrm{~Hz}, \mathrm{H} 3 \mathrm{~b}), 3.55$ (3H, s, OCH3), 2.64 (1H, ddd, $J=13.8,5.5,1.8 \mathrm{~Hz}, \mathrm{H} 9 \mathrm{a}), 2.30$ $(1 \mathrm{H}, \mathrm{dd}, J=13.8,11.0 \mathrm{~Hz}, \mathrm{H} 9 \mathrm{~b}), 0.91(9 \mathrm{H}, \mathrm{s}, t$-Bu of TBS), $0.84(9 \mathrm{H}, \mathrm{s}, t$-Bu of TBS), 0.12 (3H, s, $\mathrm{CH}_{3}$ of TBS), 0.09 (3H, s, CH3 of TBS), 0.07 (3H, s, $\mathrm{CH}_{3}$ of TBS), -0.05 (3H, s, CH3 of TBS). ${ }^{13} \mathrm{C}\left\{{ }^{1} \mathrm{H}\right\}$ NMR (125 MHz, $\left.\mathrm{CDCl}_{3}\right): \delta 190.9,171.0,165.6,148.3,131.8,130.0,129.9,128.6$ (2C), 127.1 (2C), $123.1(\mathrm{q}, J=286 \mathrm{~Hz}), 84.6(\mathrm{q}, J=28.7 \mathrm{~Hz}), 83.6,69.6,68.4,60.0,58.2,55.4,29.8,25.7$ (3C), 25.5 (3C), 18.2, 17.8, -4.7, -5.2, -5.4, -5.5. HRMS (ESI-TOF) $[\mathrm{M}+\mathrm{Na}]^{+} \mathrm{m} / \mathrm{z}$ : Calcd for $\mathrm{C}_{32} \mathrm{H}_{47} \mathrm{O}_{8} \mathrm{~F}_{3} \mathrm{Si}_{2} \mathrm{Na}$ 695.2654; Found 695.2629.

(R)-MTPA ester S11b. $(S)$-(+)- $\alpha$-Methoxy- $\alpha$-(trifluoromethyl)phenylacetyl chloride $(14.0 \mu \mathrm{L}, 74.8$ $\mu \mathrm{mol})$ and DMAP $(0.6 \mathrm{mg}, 5 \mu \mathrm{mol})$ were successively added to a solution of enone $\mathbf{1 8}(17.5 \mathrm{mg}, 38.4$ $\mu \mathrm{mol})$ and $\mathrm{Et}_{3} \mathrm{~N}(21.0 \mu \mathrm{L}, 152 \mu \mathrm{mol})$ in $\mathrm{CH}_{2} \mathrm{Cl}_{2}(380 \mu \mathrm{L})$ at $25^{\circ} \mathrm{C}$. After being stirred at $25^{\circ} \mathrm{C}$ for 30 min, the reaction mixture was diluted with toluene $(3 \mathrm{~mL})$ and concentrated. The residue was purified by flash column chromatography on silica gel $(0.5 \mathrm{~g}$, hexane/EtOAc $=5 / 1)$ to afford $(R)$-MTPA ester S11b $(25.5 \mathrm{mg}, 37.9 \mu \mathrm{mol})$ in $99 \%$ yield: colorless oil. $[\alpha]_{\mathrm{D}}{ }^{24}+25.9$ (c 1.28, $\left.\mathrm{CHCl}_{3}\right)$. IR (film): 2939 , 2859, 1767, 1687, 1463, 1255, 1178, 1119, 1058, $1014 \mathrm{~cm}^{-1} .{ }^{1} \mathrm{H}$ NMR (500 MHz, $\left.\mathrm{CDCl}_{3}\right): \delta 7.52-7.51$ (2H, m, aromatic), 7.44-7.39 (3H, m, aromatic), 6.97 (1H, ddd, $J=10.3,2.3,1.7 \mathrm{~Hz}, \mathrm{H} 7), 6.24(1 \mathrm{H}$, 
dd, $J=10.3,2.3 \mathrm{~Hz}, \mathrm{H} 6), 6.16$ (1H, dddd, $J=10.9,5.7,2.3,1.7 \mathrm{~Hz}, \mathrm{H} 8), 5.41(1 \mathrm{H}, \mathrm{d}, J=8.1 \mathrm{~Hz}, \mathrm{H} 1)$, 4.19 (1H, ddd, $J=8.1,3.5,2.3 \mathrm{~Hz}, \mathrm{H} 2), 4.01(1 \mathrm{H}, \mathrm{dd}, J=12.6,2.3 \mathrm{~Hz}, \mathrm{H} 3 \mathrm{a}), 3.80$ (1H, dd, $J=12.6$, $3.5 \mathrm{~Hz}, \mathrm{H} 3 \mathrm{~b}), 3.56$ (3H, s, $\left.\mathrm{OCH}_{3}\right), 2.59$ (1H, ddd, $\left.J=13.8,5.7,2.3 \mathrm{~Hz}, \mathrm{H} 9 \mathrm{a}\right), 2.18$ (1H, dd, $J=13.8$, $10.9 \mathrm{~Hz}, \mathrm{H} 9 \mathrm{~b}), 0.91$ (9H, s, $t$-Bu of TBS), 0.81 (9H, s, $t$-Bu of TBS), 0.10 (3H, s, CH3 of TBS), 0.09 (3H, s, $\mathrm{CH}_{3}$ of TBS), 0.07 (3H, s, $\mathrm{CH}_{3}$ of TBS), -0.09 (3H, s, CH3 of TBS). ${ }^{13} \mathrm{C}\left\{{ }^{1} \mathrm{H}\right\}$ NMR $(125 \mathrm{MHz}$, $\left.\mathrm{CDCl}_{3}\right): \delta 191.0,171.1,165.7,148.2,131.7,130.1,129.9,128.6(2 \mathrm{C}), 127.1(2 \mathrm{C}), 123.1$ (q, $J=290$ Hz), 84.7 (q, $J=28.6 \mathrm{~Hz}), 83.6,69.7,68.3,60.0,58.2,55.5,29.5,25.7$ (3C), 25.5 (3C), 18.2, 17.7, 4.7, -5.2, -5.4, -5.5. HRMS (ESI-TOF) $[\mathrm{M}+\mathrm{Na}]^{+} \mathrm{m} / \mathrm{z}$ : Calcd for $\mathrm{C}_{32} \mathrm{H}_{47} \mathrm{O}_{8} \mathrm{~F}_{3} \mathrm{Si}_{2} \mathrm{Na}$ 695.2654; Found 695.2629 . 
7. Investigation of C-ring construction

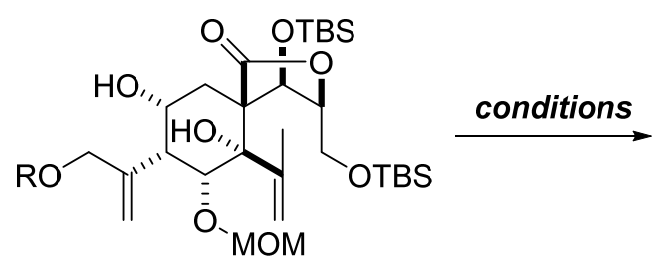

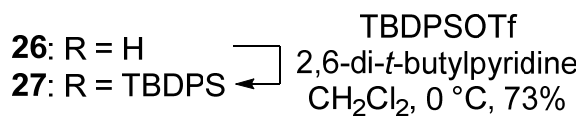

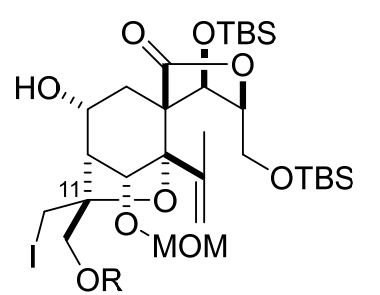

S12: $R=H$

29: $R=$ TBDPS

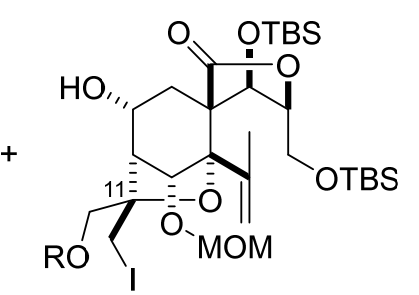

C11-epi-S12: $\mathrm{R}=\mathrm{H}$ C11-epi-29: $R=$ TBDPS

Table S4. Investigation of C-ring construction

\begin{tabular}{|c|c|c|c|}
\hline entry & SM & conditions & results \\
\hline 1 & 26 & $\mathrm{I}_{2}, \mathrm{NaHCO}_{3}, \mathrm{MeCN}, 0{ }^{\circ} \mathrm{C}, 10 \mathrm{~min}$ & C11-epi-S12: $41 \%$ \\
\hline 2 & 27 & $\mathrm{I}_{2}, \mathrm{NaHCO}_{3}, \mathrm{MeCN}, 0^{\circ} \mathrm{C}, 10 \mathrm{~min}$ & $\begin{array}{l}\text { 29: } 22 \% \\
\text { C11-epi-29: } 14 \%\end{array}$ \\
\hline 3 & 27 & $\mathrm{I}_{2}, 2,6$-di-t-butylpyridine, $\mathrm{CH}_{2} \mathrm{Cl}_{2},-50{ }^{\circ} \mathrm{C}, 15 \mathrm{~h}$ & 29: $85 \%$ \\
\hline 4 & 26 & $\begin{array}{l}\text { TBDPSOTf, 2,6-di-t-butylpyridine, } \mathrm{CH}_{2} \mathrm{Cl}_{2}, 0{ }^{\circ} \mathrm{C}, 25 \mathrm{~min} \text {; } \\
\mathrm{I}_{2}, 2,6 \text {-di- } t \text {-butylpyridine, }-50{ }^{\circ} \mathrm{C}, 17 \mathrm{~h}\end{array}$ & 29: $73 \%$ \\
\hline
\end{tabular}




\section{References}

S1 Urabe, D.; Yamaguchi, H.; Someya, A. Inoue, M. Intermolecular Radical Reaction of $O$,Se-Acetals Generated via Seleno-Pummerer Rearrangement. Org. Lett. 2012, 14, 3842-3845.

S2 Benson, W. R.; McBee, E. T.; Rand, L. N-Iodosuccinimide. Org. Synth. 1962, 42, 73-75.

S3 Núñez, M. J.; Cortés-Selva, F.; Bazzocchi, I. L.; Jiménez, I. A.; González A. G.; Ravelo, A. G.; Gavin, J. A. Absolute Configuration and Complete Assignment of ${ }^{13} \mathrm{C}$ NMR Data for New Sesquiterpenes from Maytenus chiapensis. J. Nat. Prod. 2003, 66, 572-574.

S4 Tomanik, M.; Xu, S.; Herzon, S. B. Enantioselective Synthesis of Euonyminol. J. Am. Chem. Soc. 2021, 143, 699-704.

S5 Ohtani, I.; Kusumi, T.; Kashman, Y.; Kakisawa, H. High-field FT NMR application of Mosher's method. The absolute configurations of marine terpenoids. J. Am. Chem. Soc. 1991, 113, 4092-4096. 
<smiles>C=C(C(=O)OCC)[C@H](O)C1COC(C)(C)O1</smiles>

crude $13 / C 1$-epi-13 = 2.8:1

${ }^{1} \mathrm{HNMR}\left(500 \mathrm{MHz}, \mathrm{CDCl}_{3}\right)$

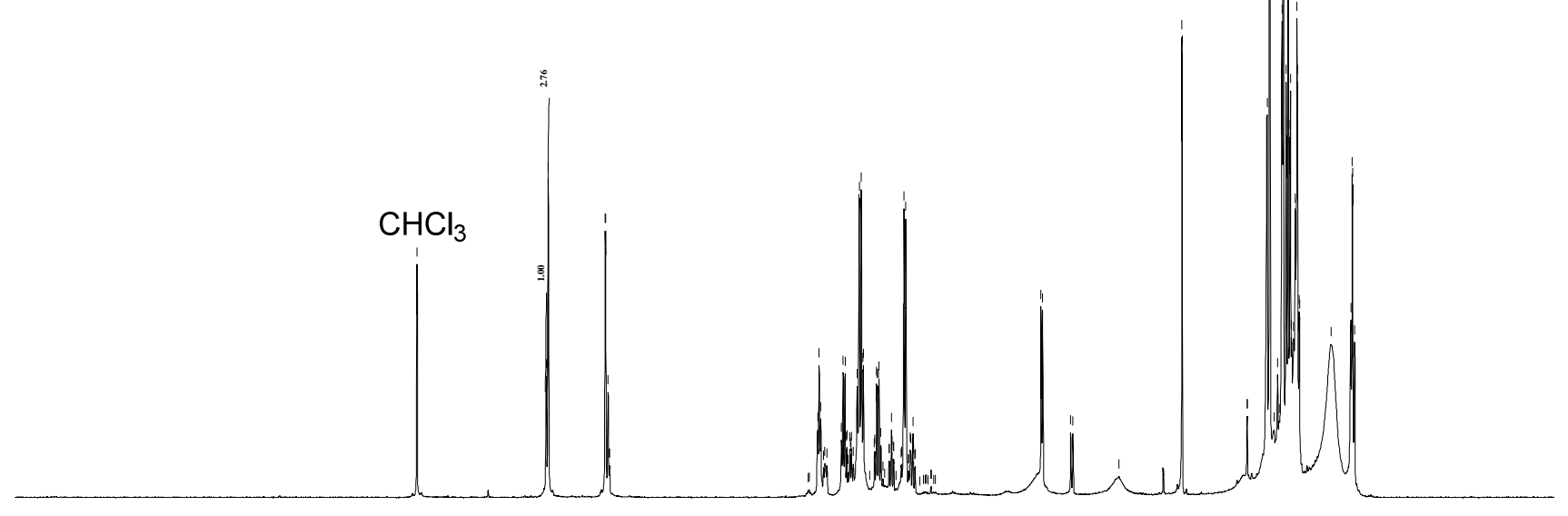

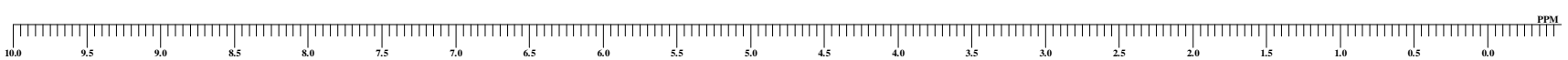


Supporting Information<smiles>C=C(C(=O)OCC)[C@@H](O)C1COC(C)(C)O1</smiles>

13

${ }^{1} \mathrm{H}$ NMR $\left(500 \mathrm{MHz}, \mathrm{CDCl}_{3}\right)$
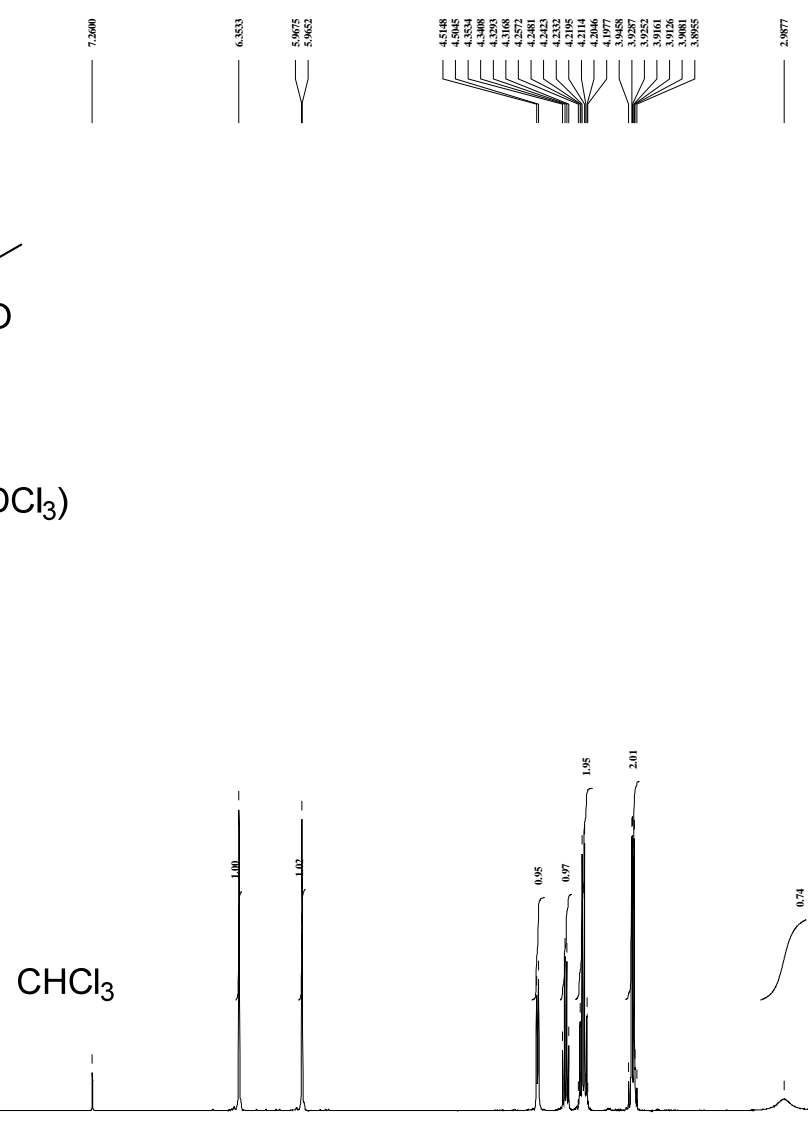

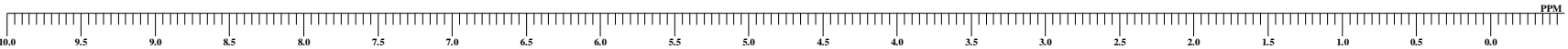<smiles>C=C(OCC)C(=C)[C@@H](O)C1COC(C)(C)O1</smiles>

$\mathrm{CDCl}_{3}$

${ }^{13} \mathrm{C}\left\{{ }^{1} \mathrm{H}\right\} \operatorname{NMR}\left(125 \mathrm{MHz}, \mathrm{CDCl}_{3}\right)$

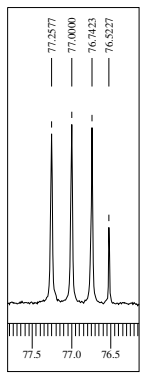

Wing

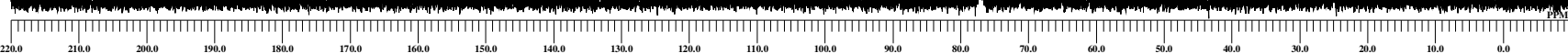

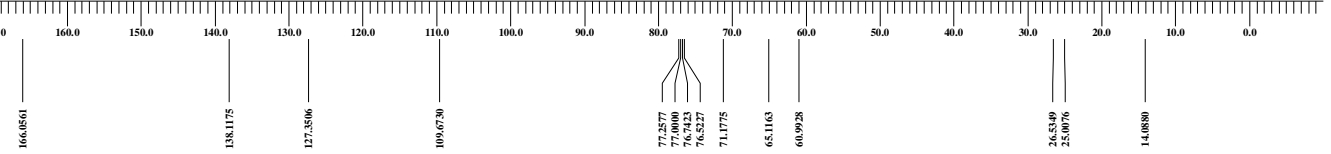


Supporting Information

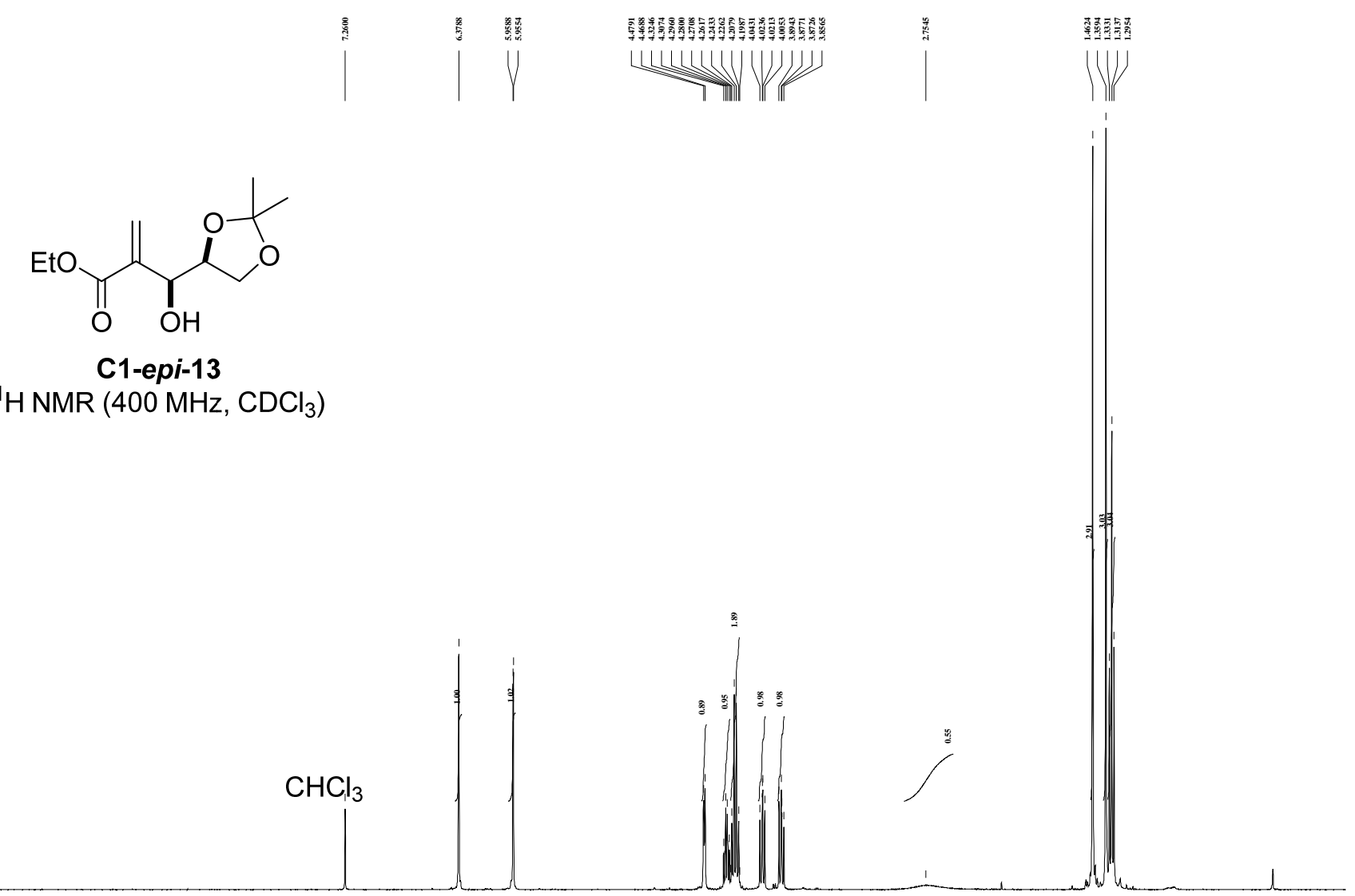

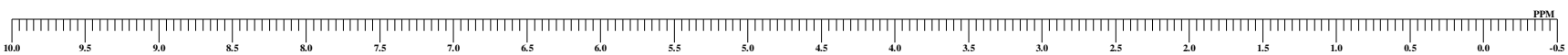<smiles>C=C(C(=O)OCC)C(O)C1COC(C)(C)O1</smiles>

$\mathrm{CDCl}_{3}$

$$
\text { C1-epi-13 }
$$

${ }^{13} \mathrm{C}\left\{{ }^{1} \mathrm{H}\right\} \operatorname{NMR}\left(100 \mathrm{MHz}, \mathrm{CDCl}_{3}\right)$

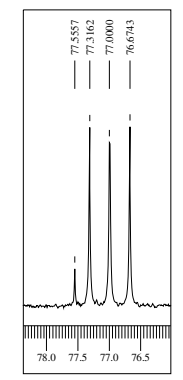

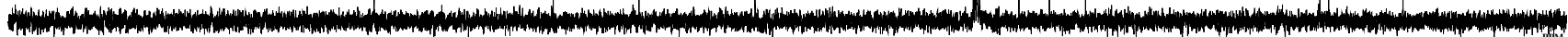

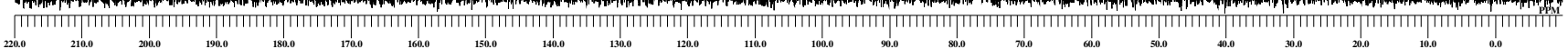

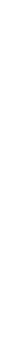



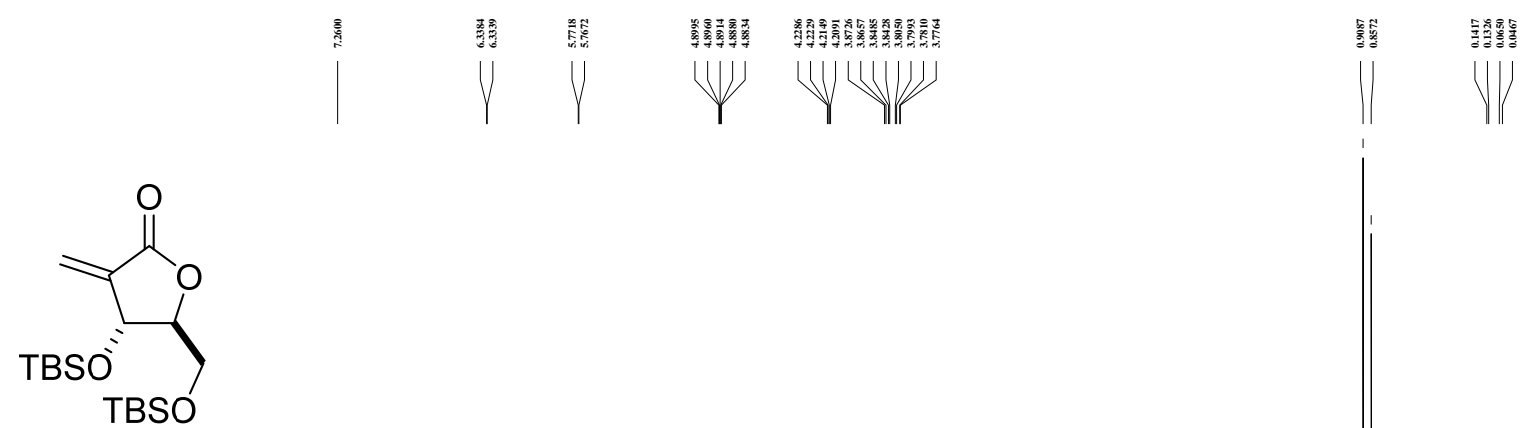

10

${ }^{1} \mathrm{H} \mathrm{NMR}\left(500 \mathrm{MHz}, \mathrm{CDCl}_{3}\right)$

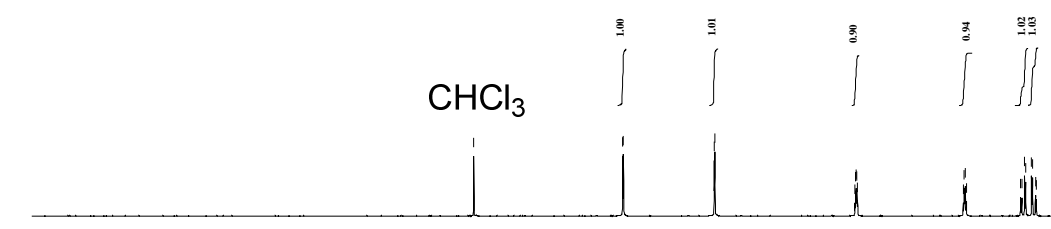

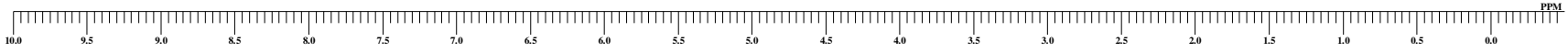<smiles>C=C1C(=O)OC(COC(C)(C)C)C1[Se-](C)(F)F</smiles>

$\mathrm{CDCl}_{3}$

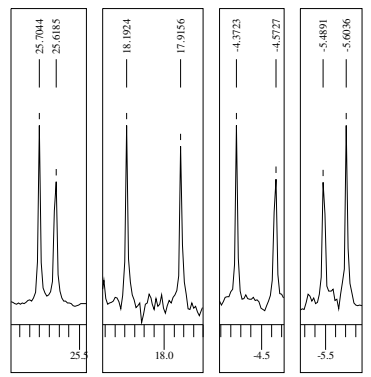

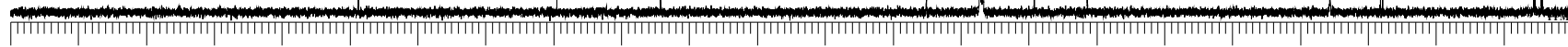

$$
10
$$

${ }^{13} \mathrm{C}\left\{{ }^{1} \mathrm{H}\right\} \operatorname{NMR}\left(125 \mathrm{MHz}, \mathrm{CDCl}_{3}\right)$ 
Supporting Information

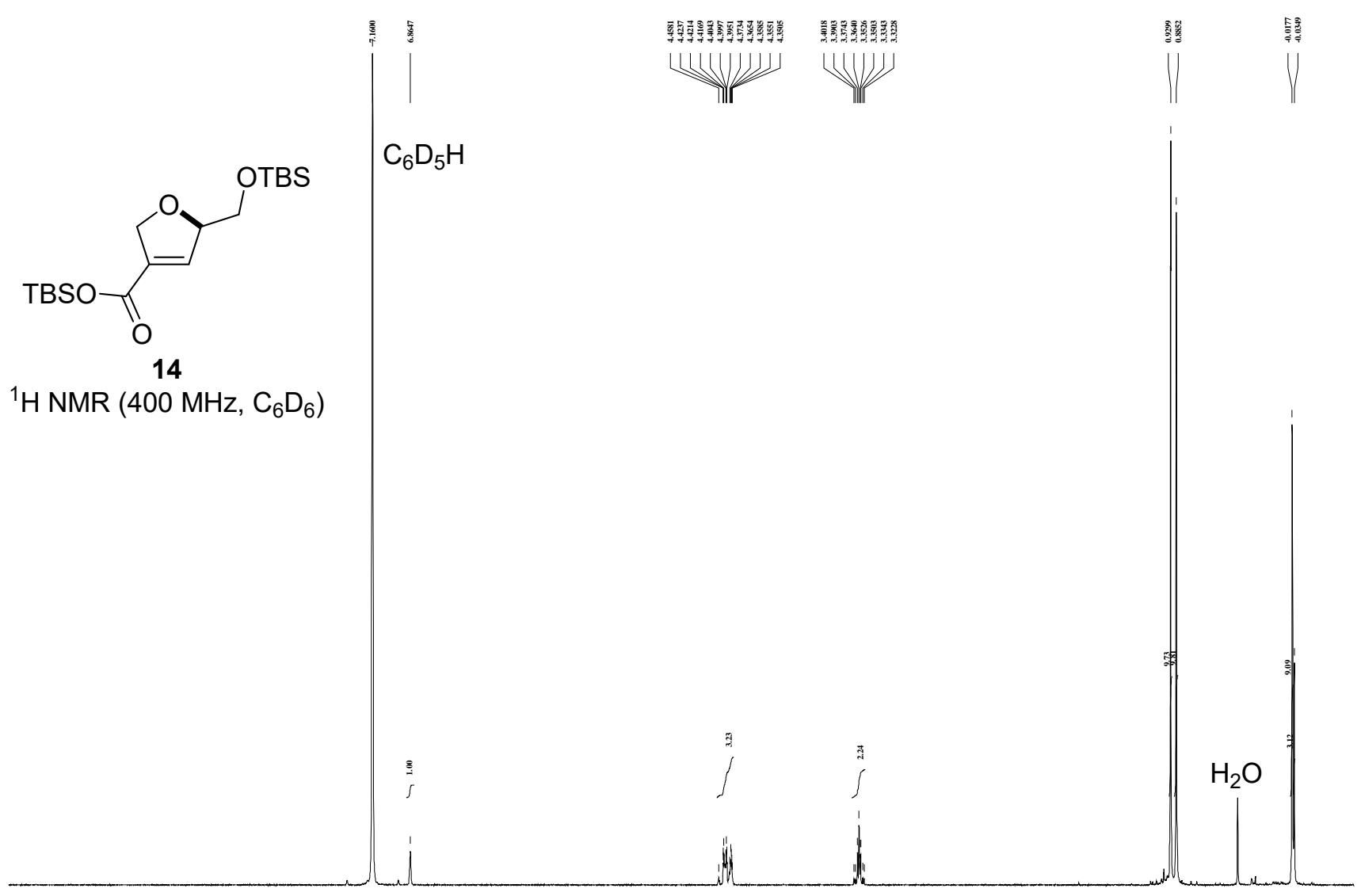

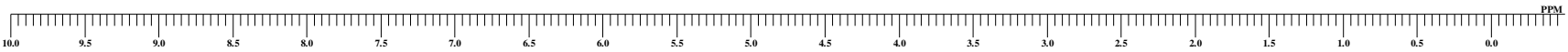

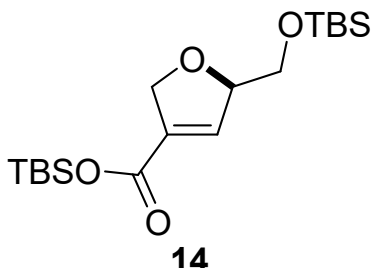

${ }^{13} \mathrm{C}\left\{{ }^{1} \mathrm{H}\right\} \operatorname{NMR}\left(125 \mathrm{MHz}, \mathrm{CDCl}_{3}\right)$

$\mathrm{CDCl}_{3}$

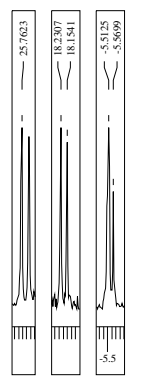

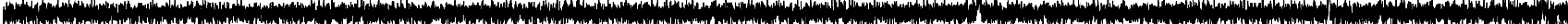

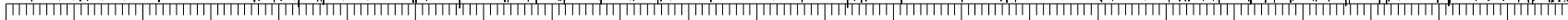

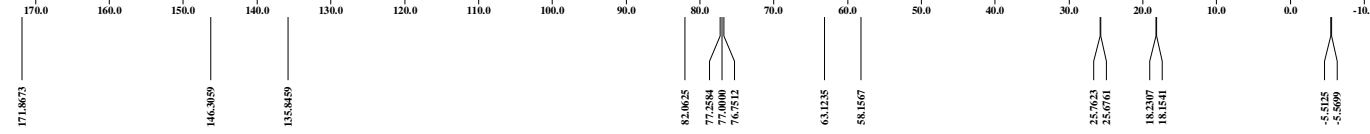




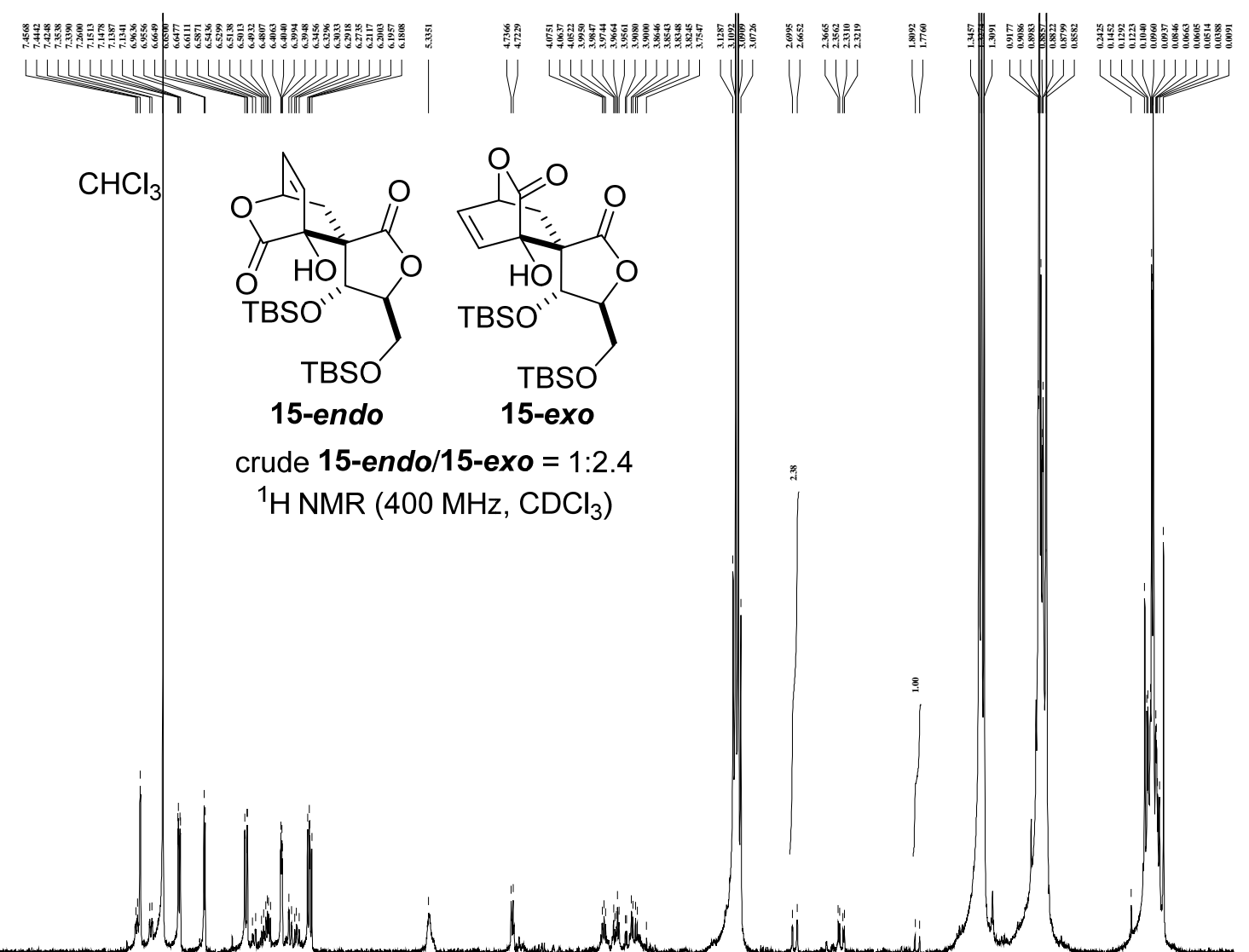

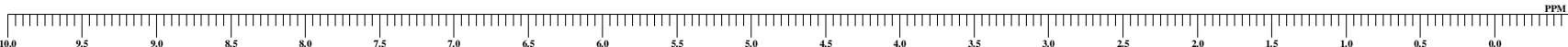




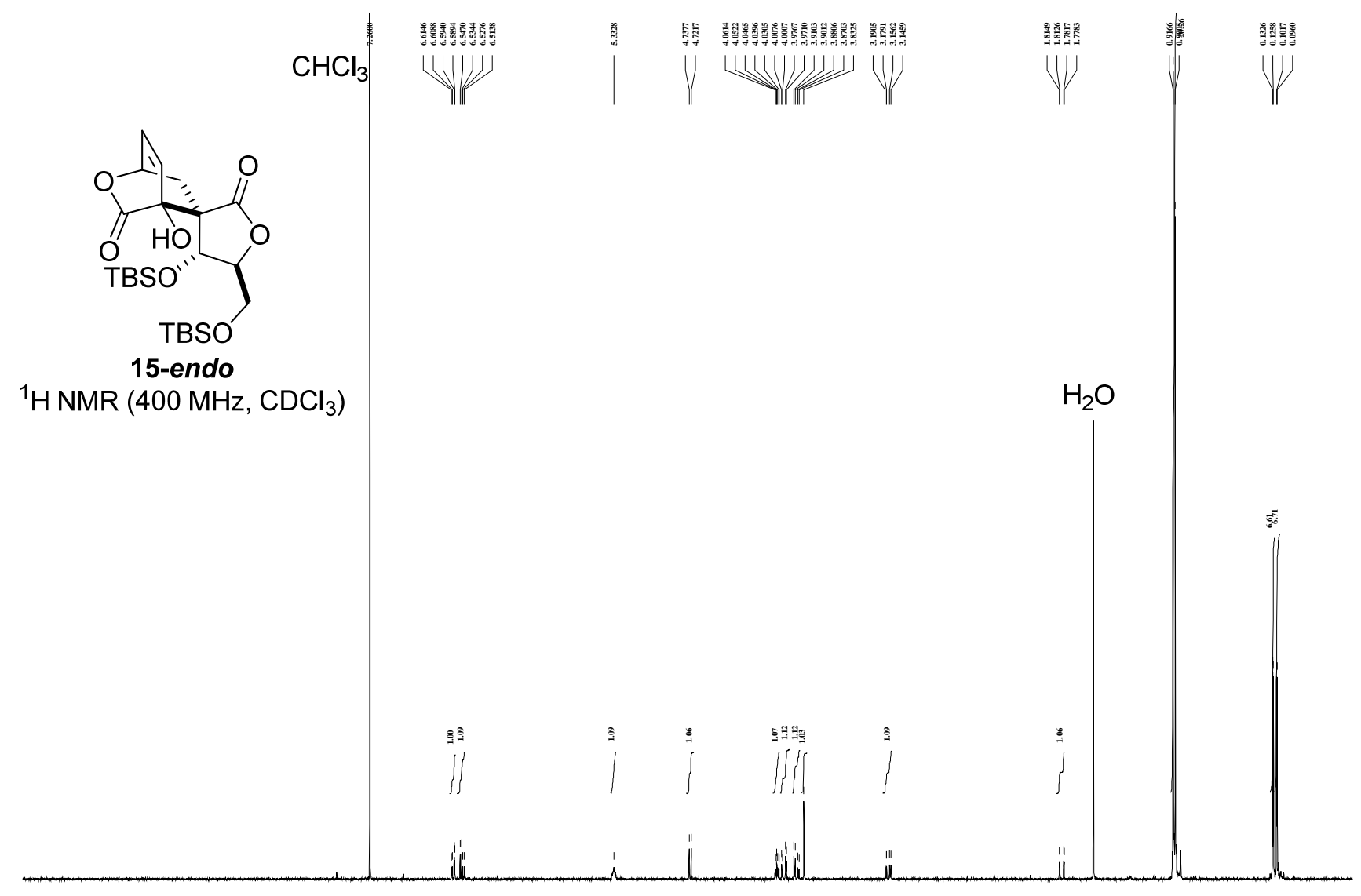

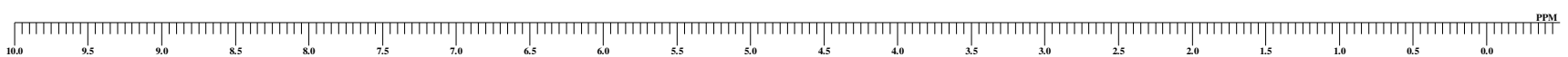

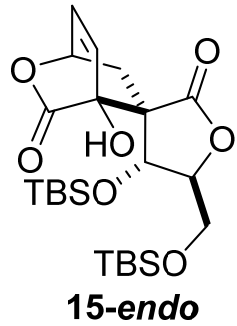

${ }^{13} \mathrm{C}\left\{{ }^{1} \mathrm{H}\right\} \mathrm{NMR}\left(100 \mathrm{MHz}, \mathrm{CDCl}_{3}\right)$
$\mathrm{CDCl}_{3}$

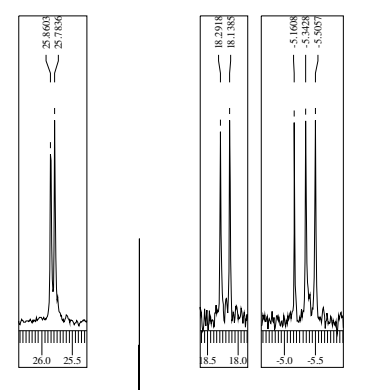


Supporting Information

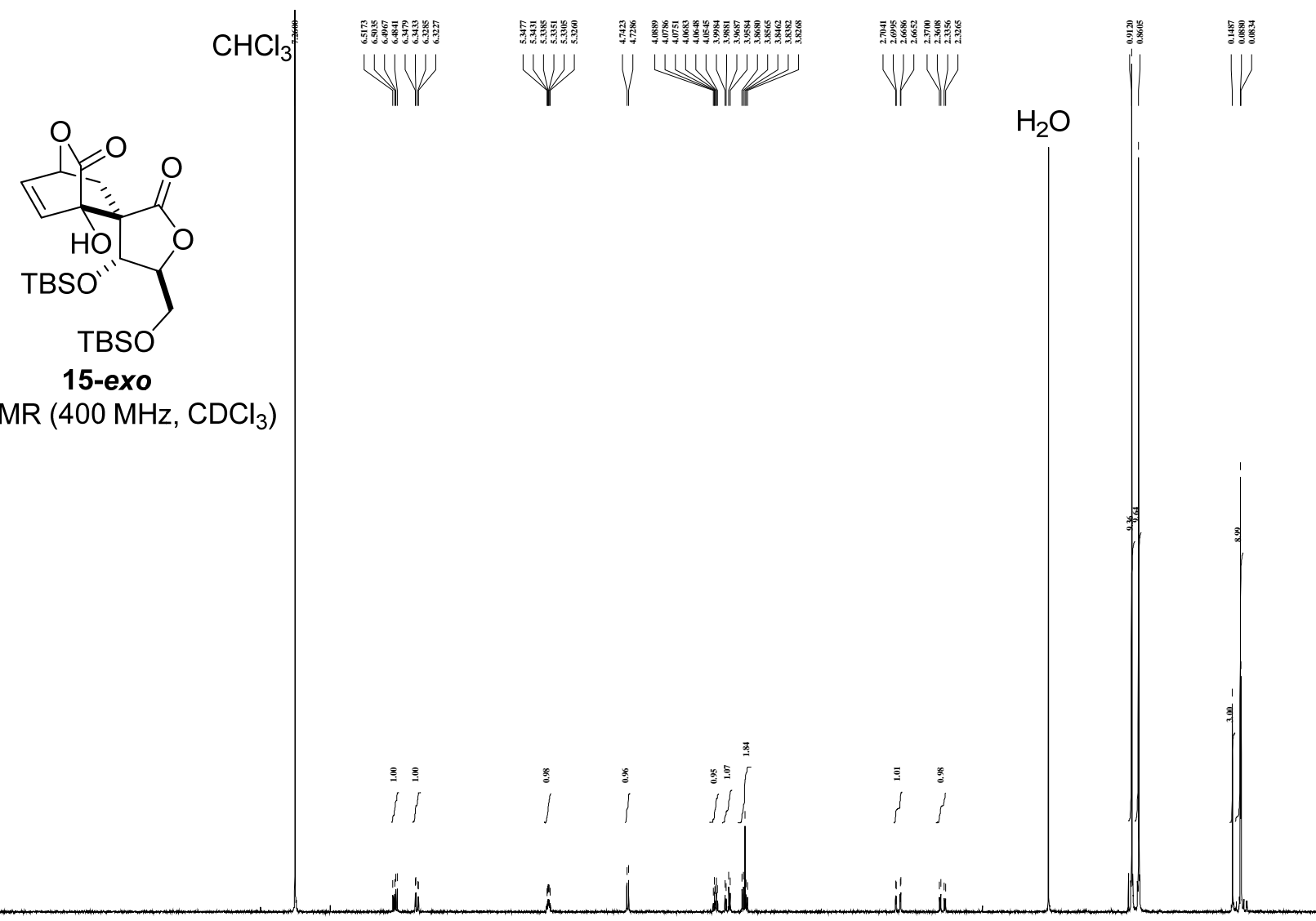

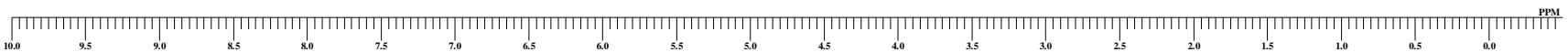

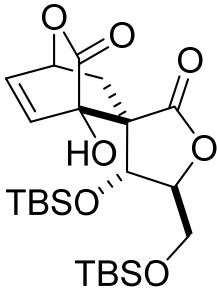

15-exo

${ }^{13} \mathrm{C}\left\{{ }^{1} \mathrm{H}\right\} \operatorname{NMR}\left(100 \mathrm{MHz}, \mathrm{CDCl}_{3}\right)$
$\mathrm{CDCl}_{3}$

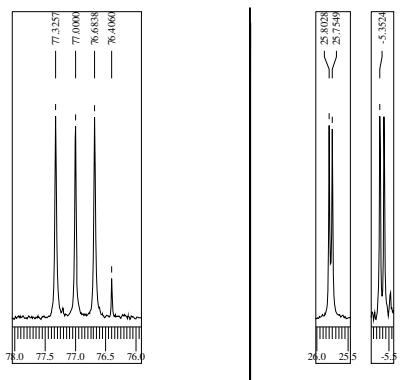




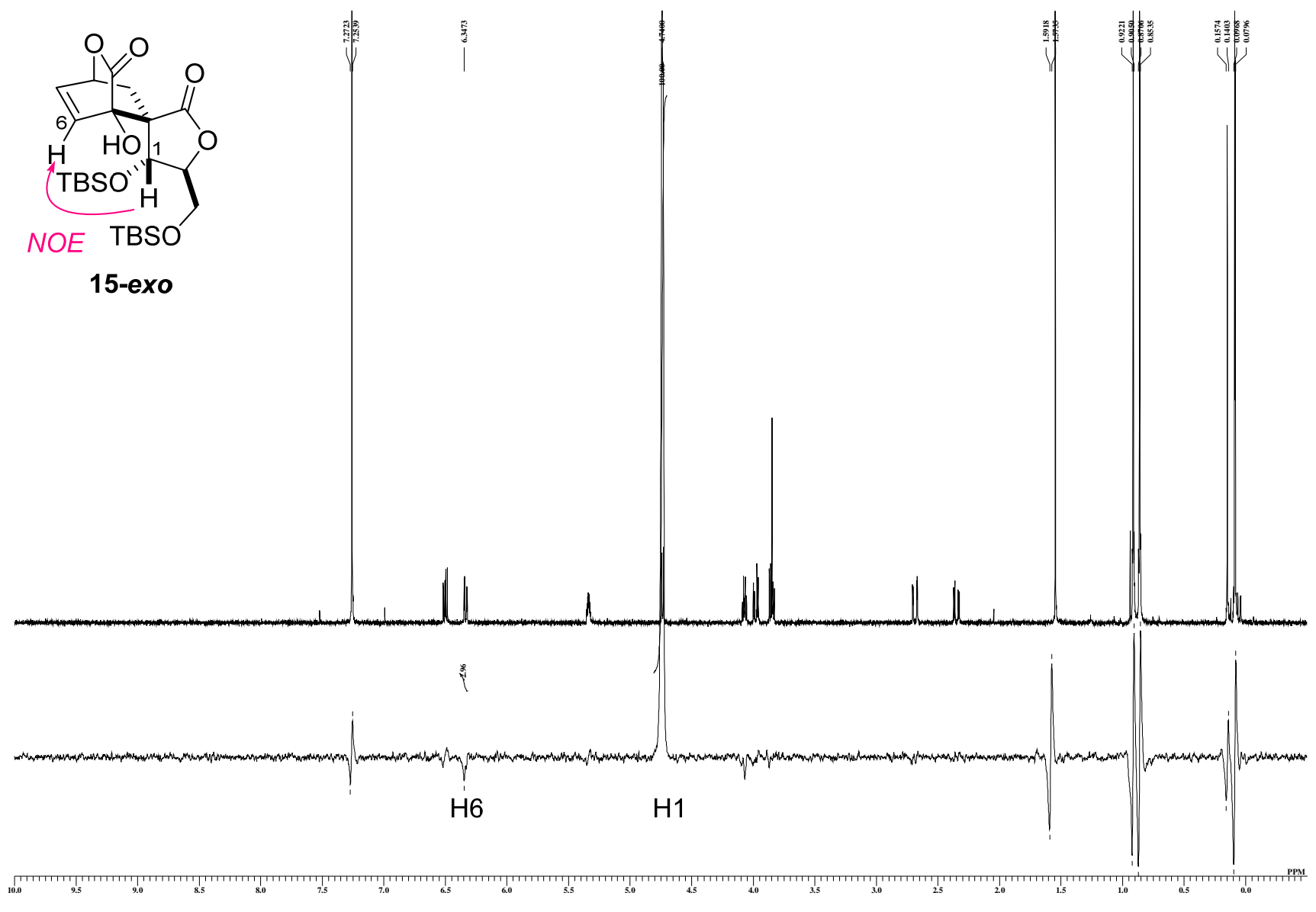




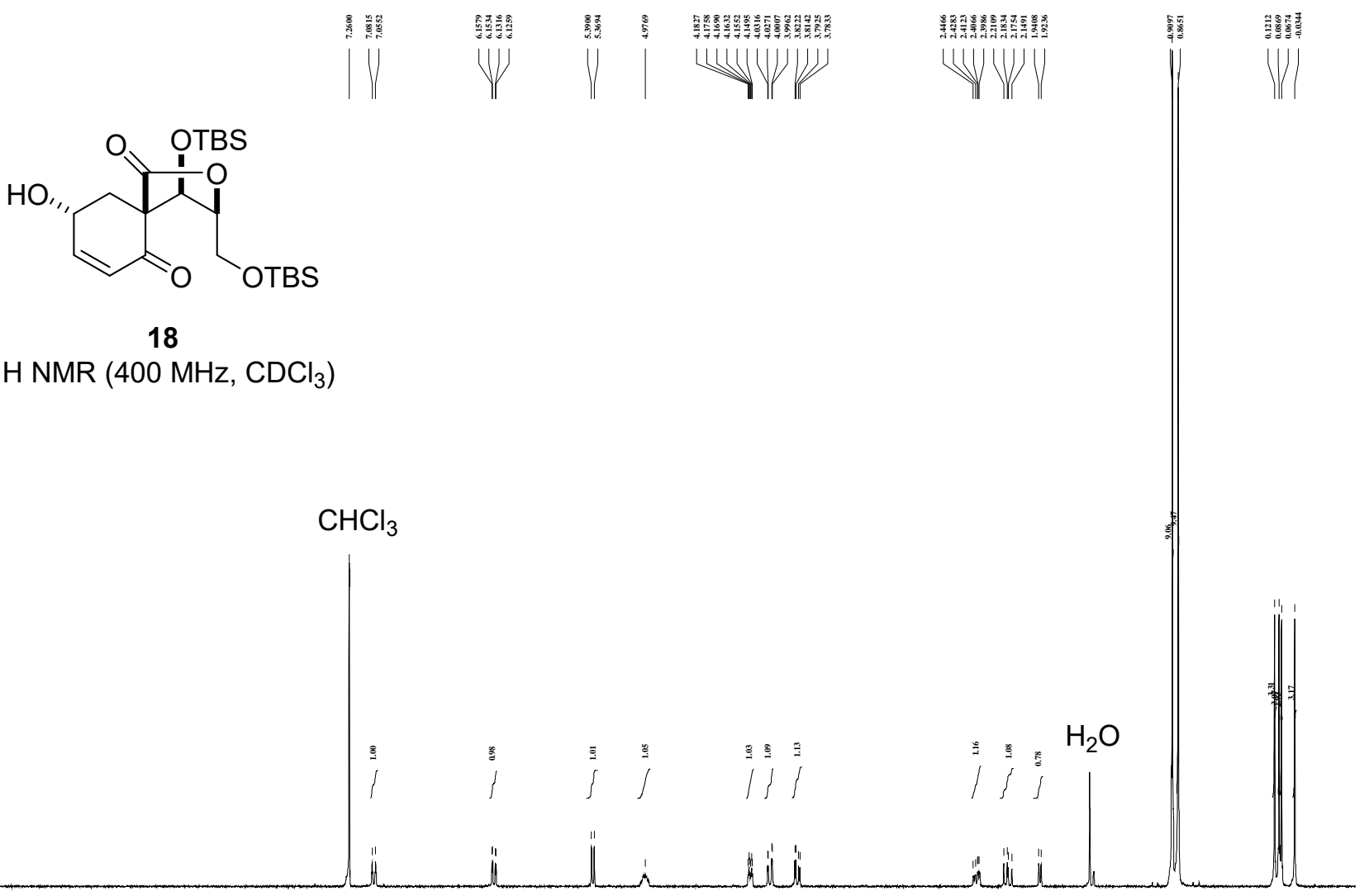

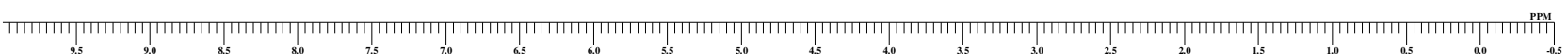

$\mathrm{CDCl}_{3}$

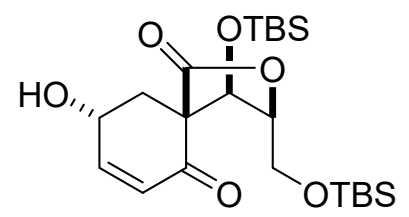

18

${ }^{13} \mathrm{C}\left\{{ }^{1} \mathrm{H}\right\} \mathrm{NMR}\left(100 \mathrm{MHz}, \mathrm{CDCl}_{3}\right)$ 


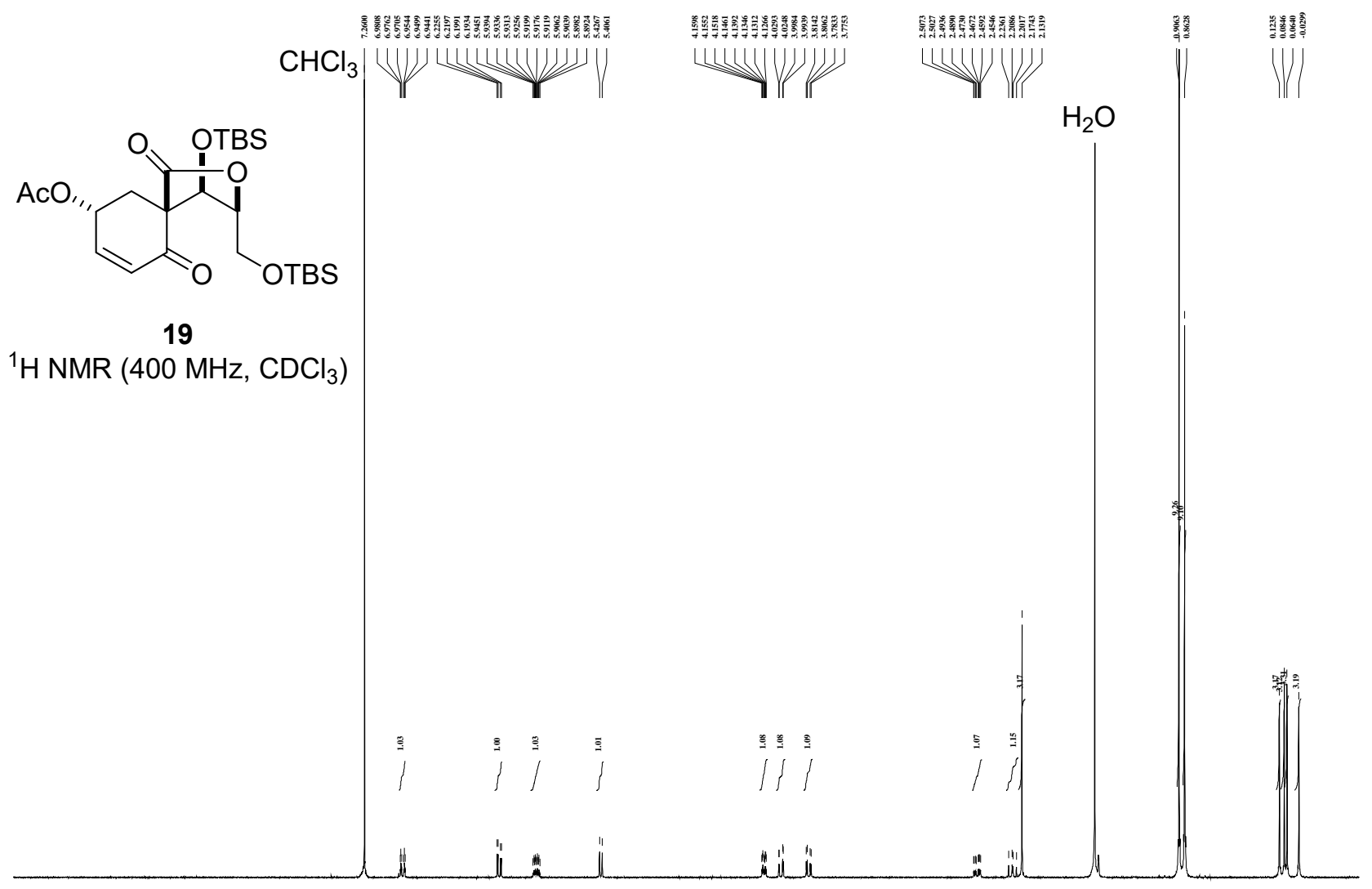

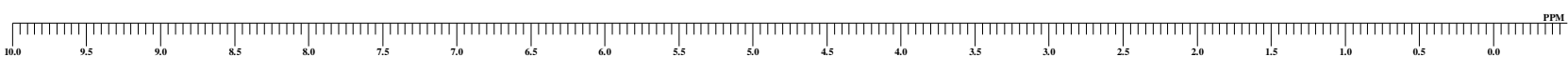

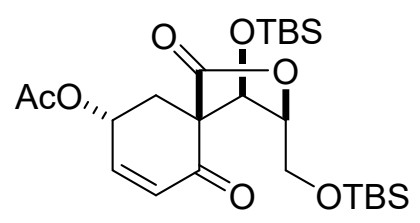

19

${ }^{13} \mathrm{C}\left\{{ }^{1} \mathrm{H}\right\} \mathrm{NMR}\left(100 \mathrm{MHz}, \mathrm{CDCl}_{3}\right)$
$\mathrm{CDCl}_{3}$
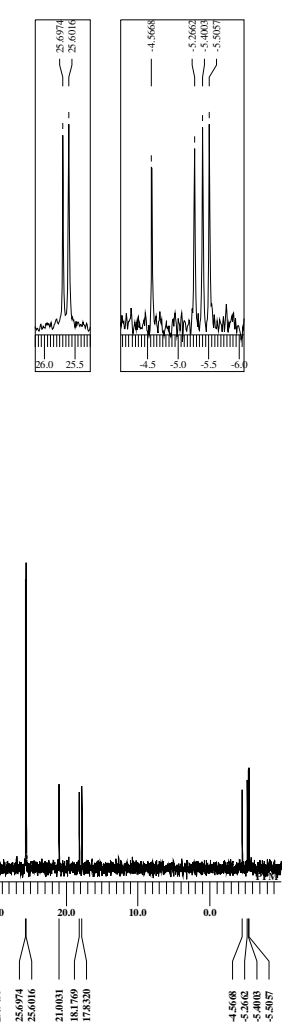
<smiles>CC(=O)OCC1CC(C(=O)[O-])(C(=O)[O-])C([Se-])O1</smiles>

20

${ }^{1} \mathrm{H} \mathrm{NMR}\left(400 \mathrm{MHz}, \mathrm{CDCl}_{3}\right)$

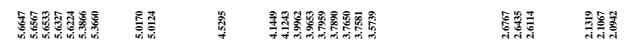

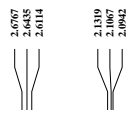

$\mathrm{CHCl}_{3}$

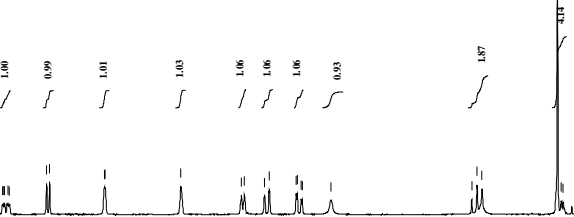

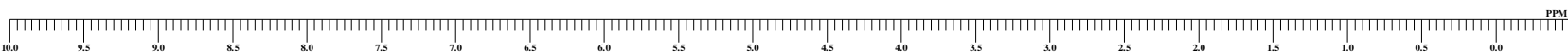

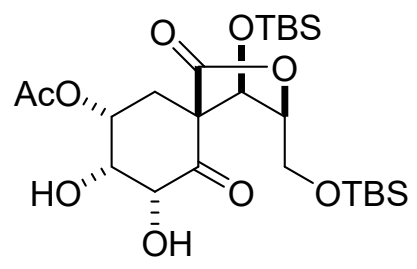

20

${ }^{13} \mathrm{C}\left\{{ }^{1} \mathrm{H}\right\} \mathrm{NMR}\left(100 \mathrm{MHz}, \mathrm{CDCl}_{3}\right)$
$\mathrm{CDCl}_{3}$

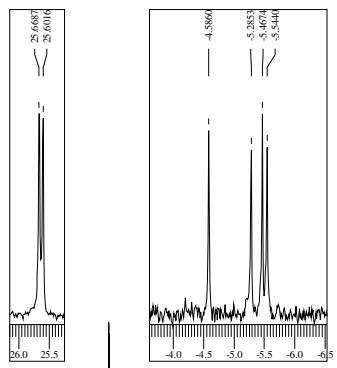

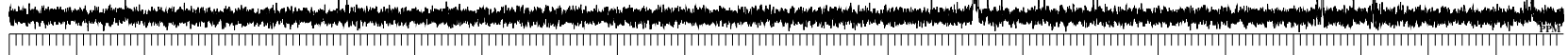



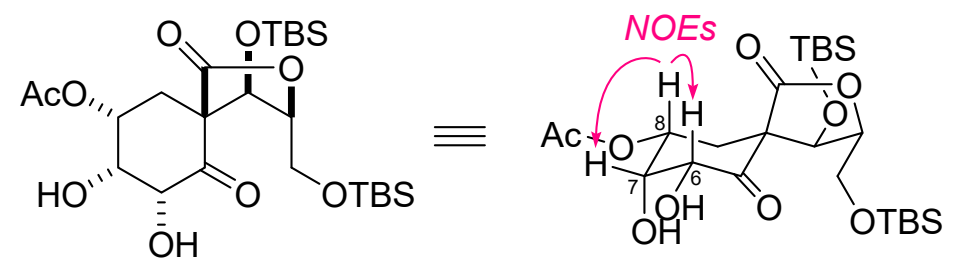

20

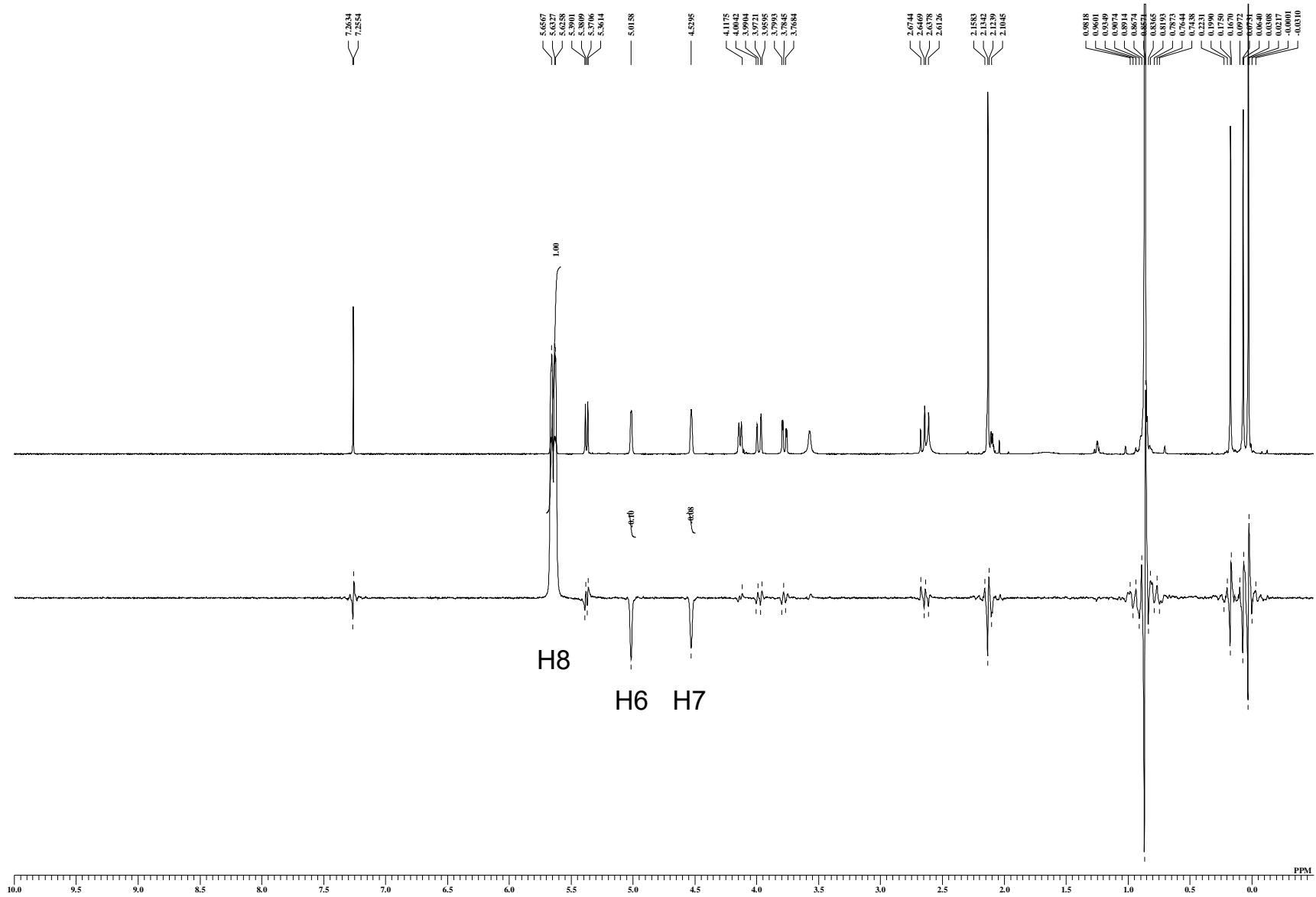




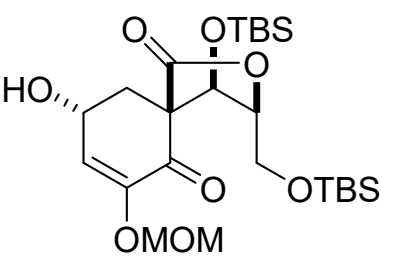

8

${ }^{1} \mathrm{H} \mathrm{NMR}\left(400 \mathrm{MHz}, \mathrm{CDCl}_{3}\right)$

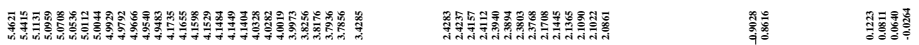
$\mathrm{H}_{2} \mathrm{O}$

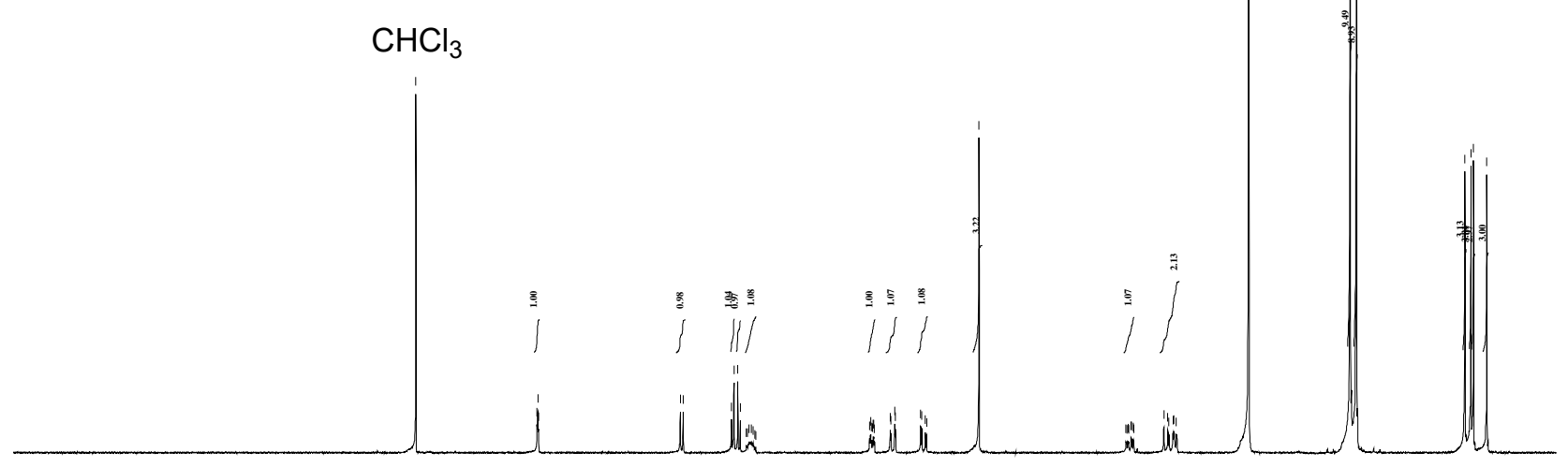

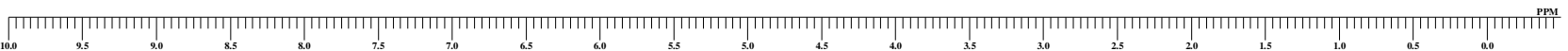

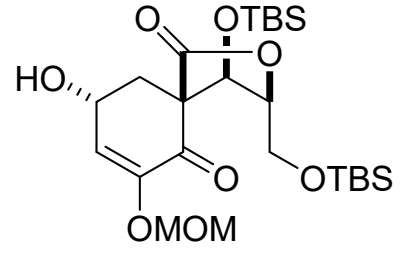

8

${ }^{13} \mathrm{C}\left\{{ }^{1} \mathrm{H}\right\} \mathrm{NMR}\left(100 \mathrm{MHz}, \mathrm{CDCl}_{3}\right)$
$\mathrm{CDCl}_{3}$

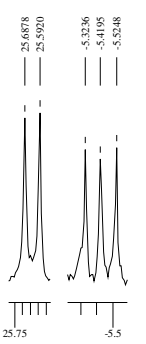

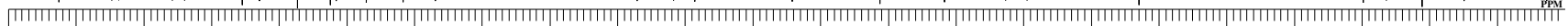

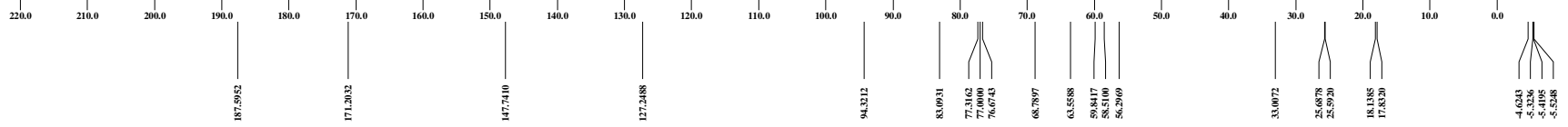


<smiles>C=C(I)[C@@H](OC)O[C@@H]1C=C(OC)C(=O)C2(C1)OC(C[O+][S-])C([O-])C2=O</smiles>

crude 23 (dr at $\mathrm{C} 13=1: 1) \quad \mathrm{CHCl}_{3}$

${ }^{1} \mathrm{H}$ NMR $\left(400 \mathrm{MHz}, \mathrm{CDCl}_{3}\right)$

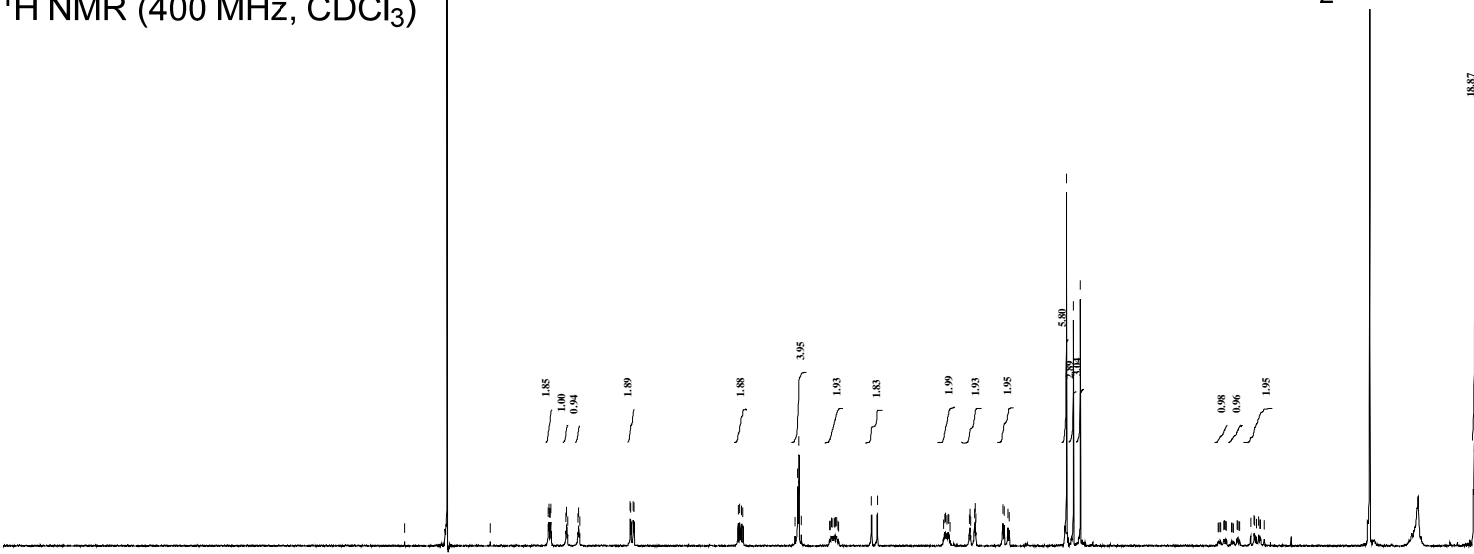

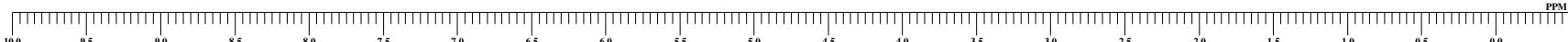


Supporting Information
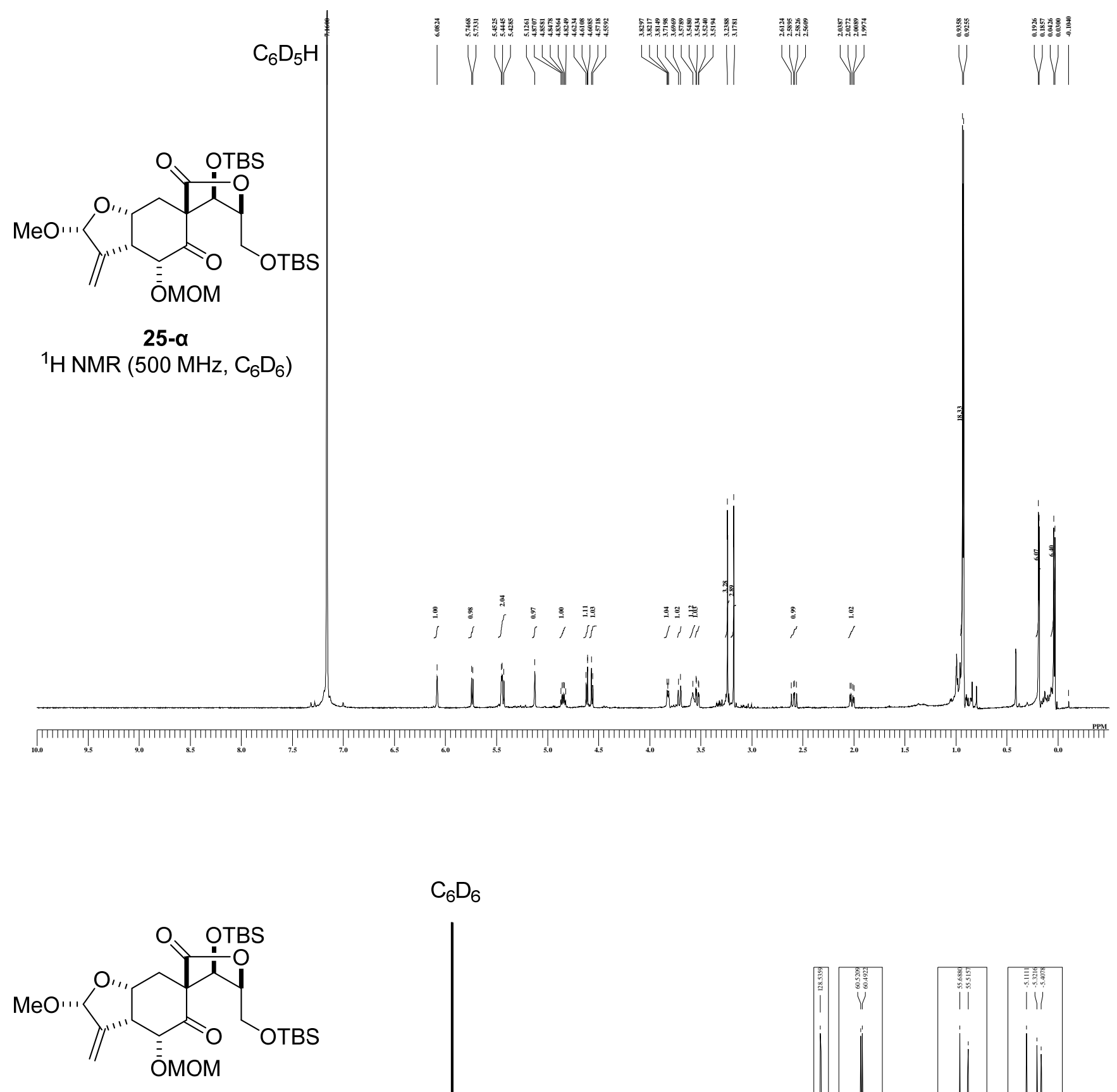

$\mathrm{C}_{6} \mathrm{D}_{6}$

${ }^{13} \mathrm{C}\left\{{ }^{1} \mathrm{H}\right\}$ NMR $\left(125 \mathrm{MHz}, \mathrm{C}_{6} \mathrm{D}_{6}\right)$
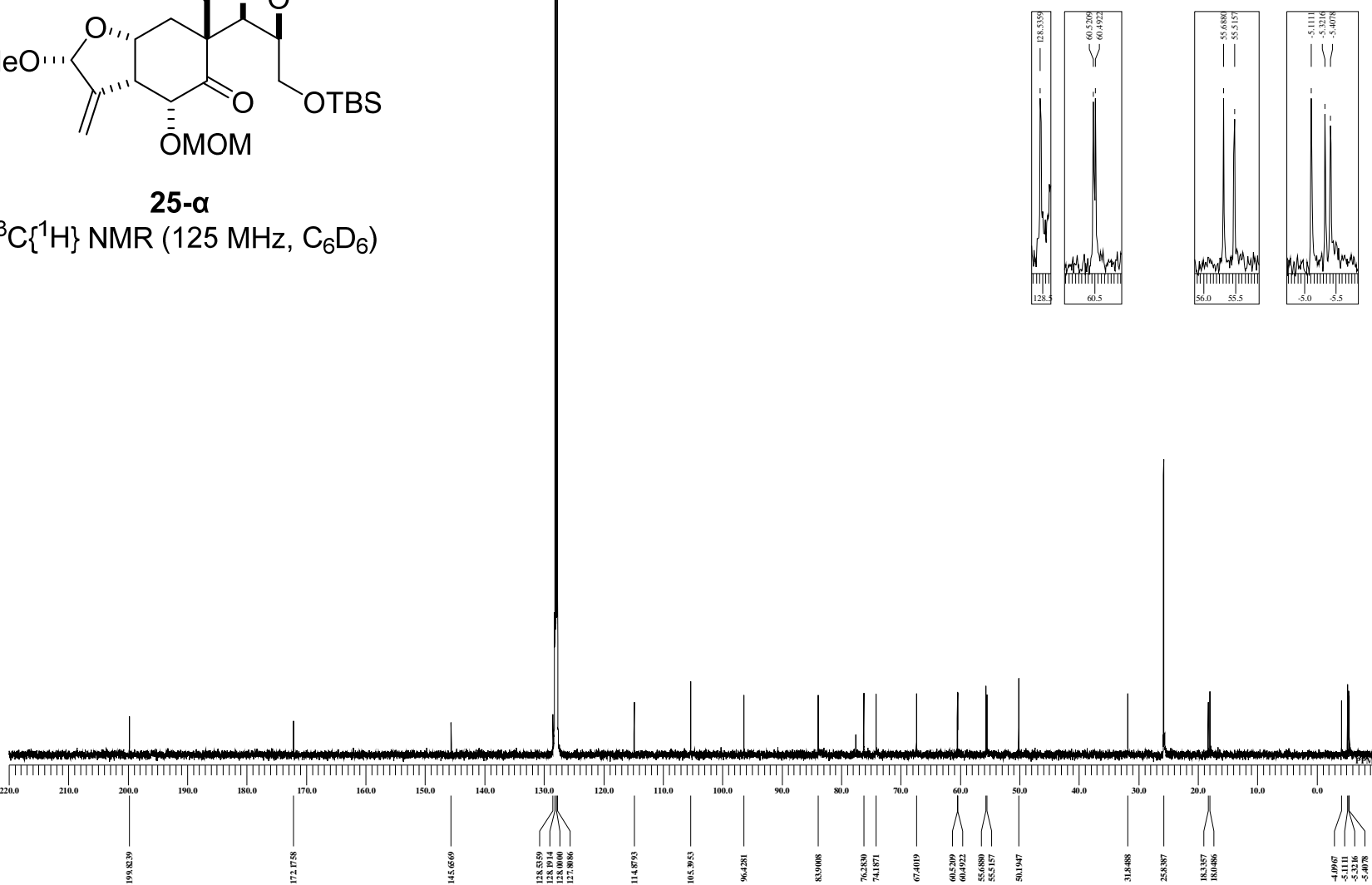

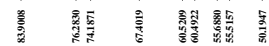

旁镂 


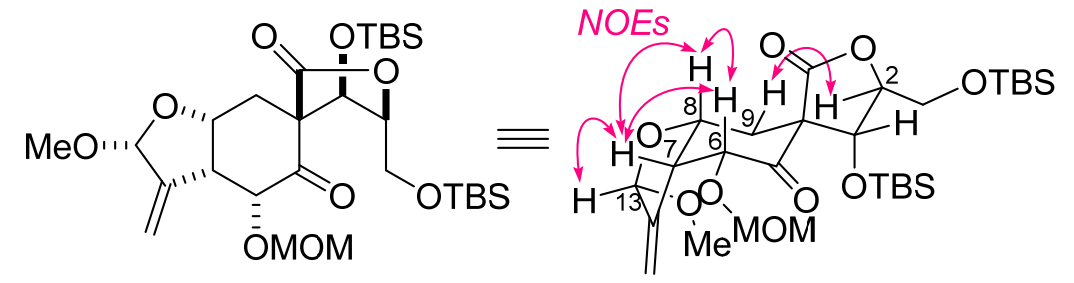

25- $\alpha$

NOESY $\left(500 \mathrm{MHz}, \mathrm{C}_{6} \mathrm{D}_{6}\right)$

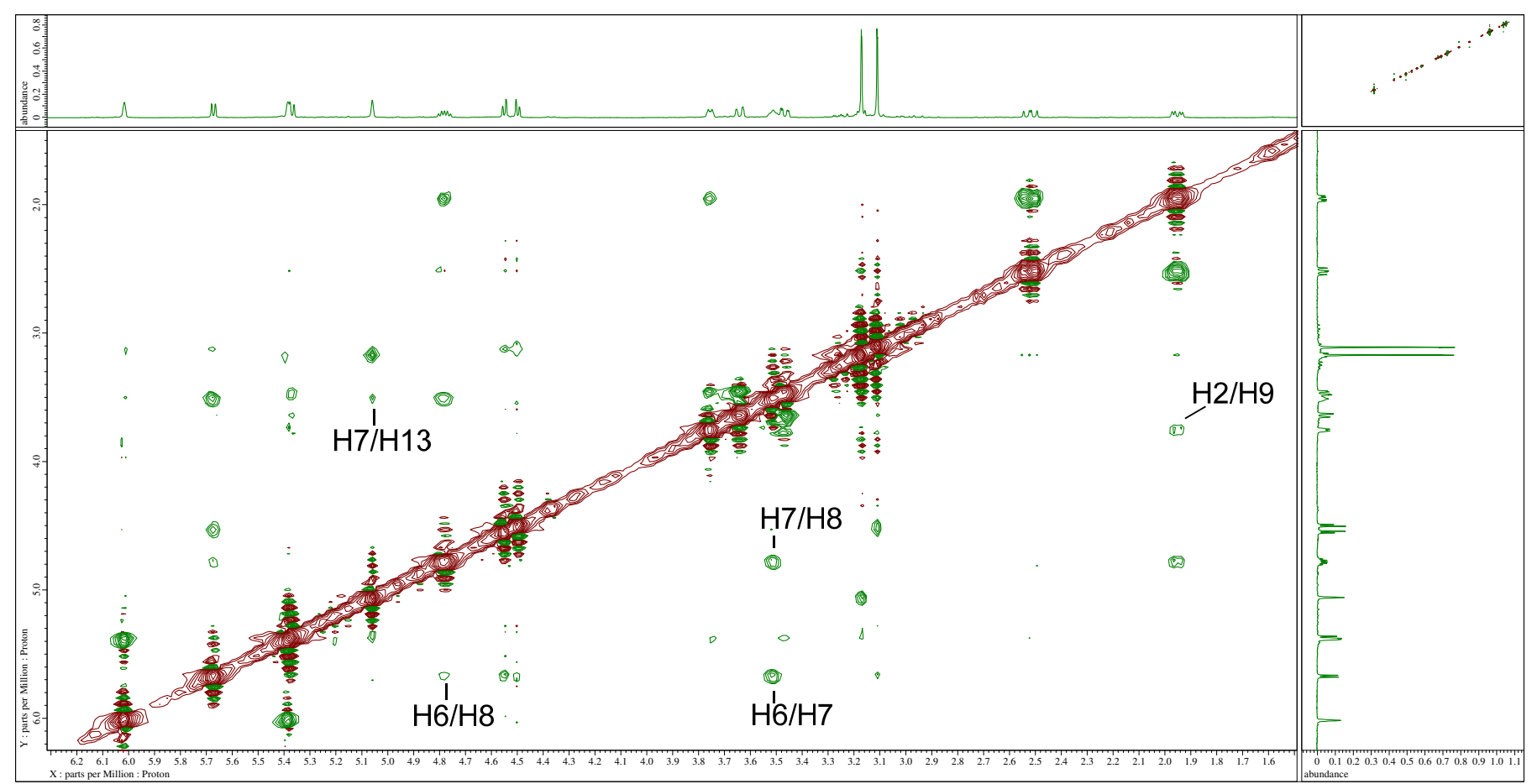




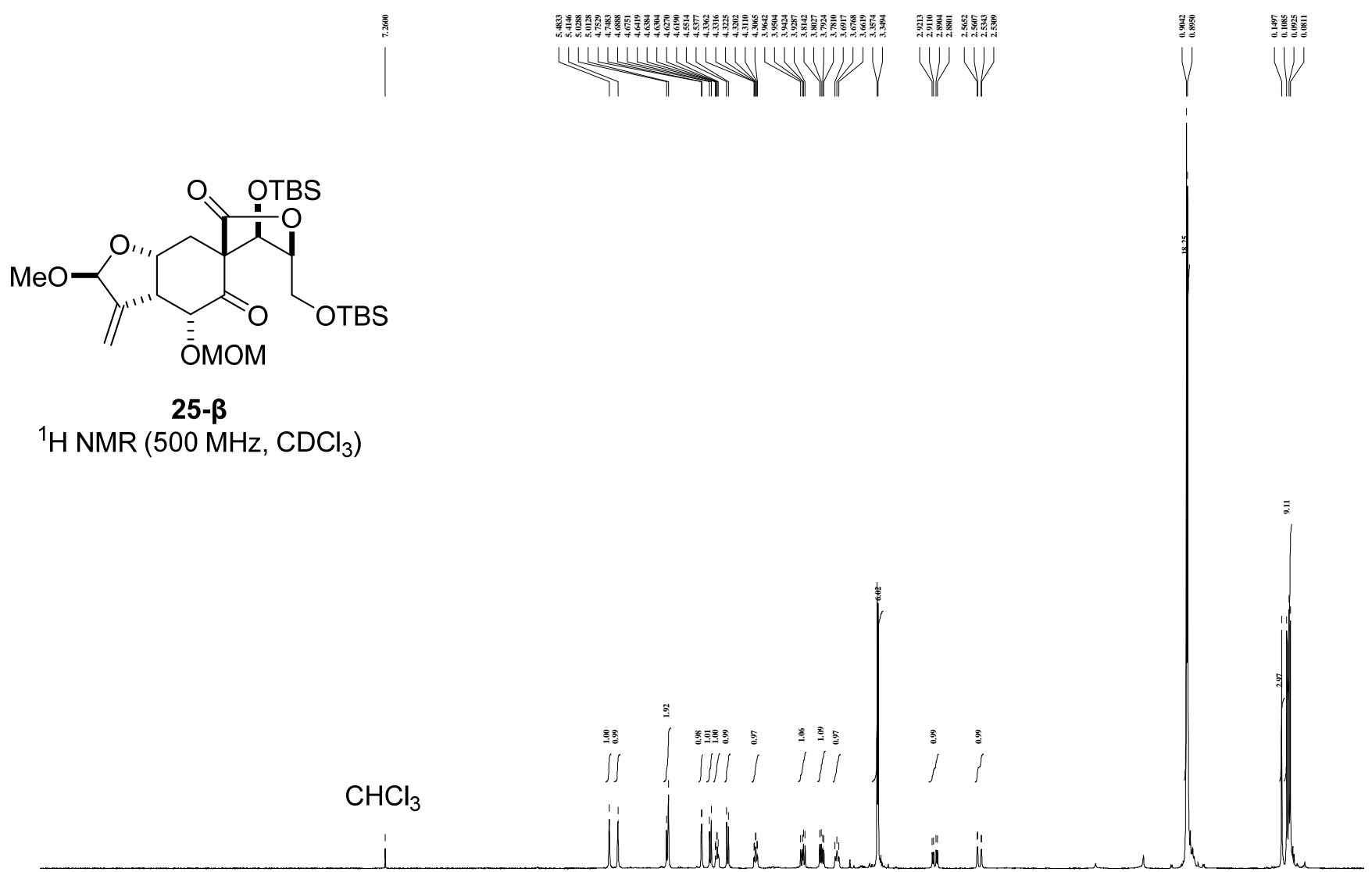

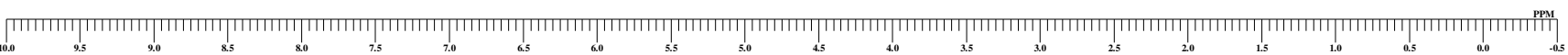<smiles></smiles>

25- $\beta$

${ }^{13} \mathrm{C}\left\{{ }^{1} \mathrm{H}\right\}$ NMR $\left(125 \mathrm{MHz}, \mathrm{CDCl}_{3}\right)$

$\mathrm{CDCl}_{3}$

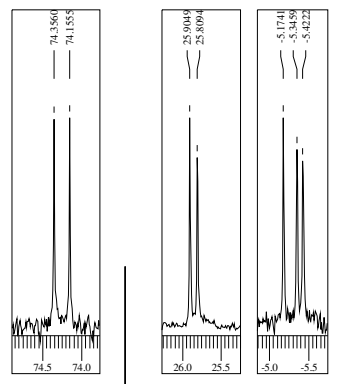

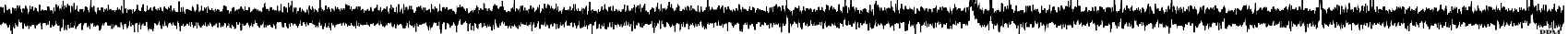

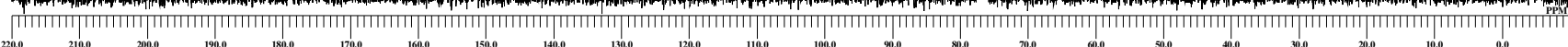

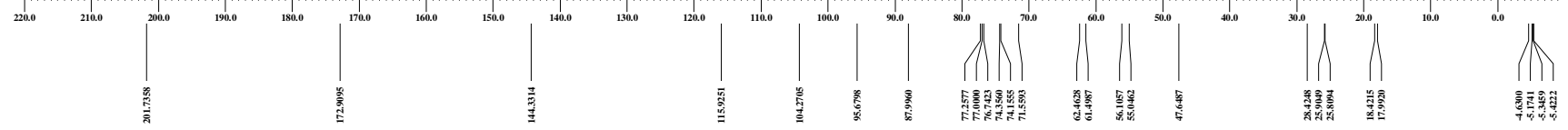




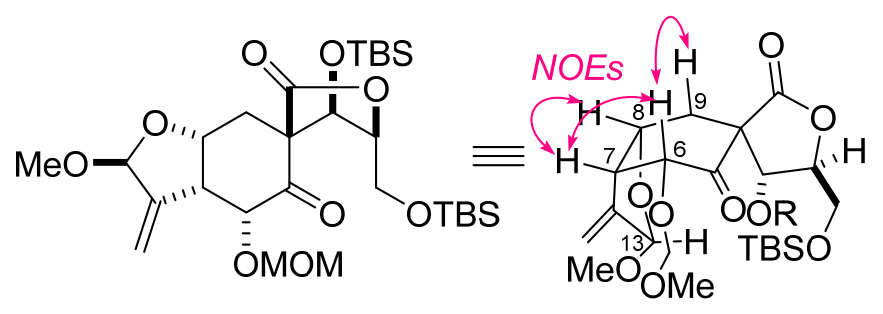

25- $\boldsymbol{\beta}$ ( $\mathrm{R}=\mathrm{TBS})$

NOESY $\left(500 \mathrm{MHz}, \mathrm{CDCl}_{3}\right)$

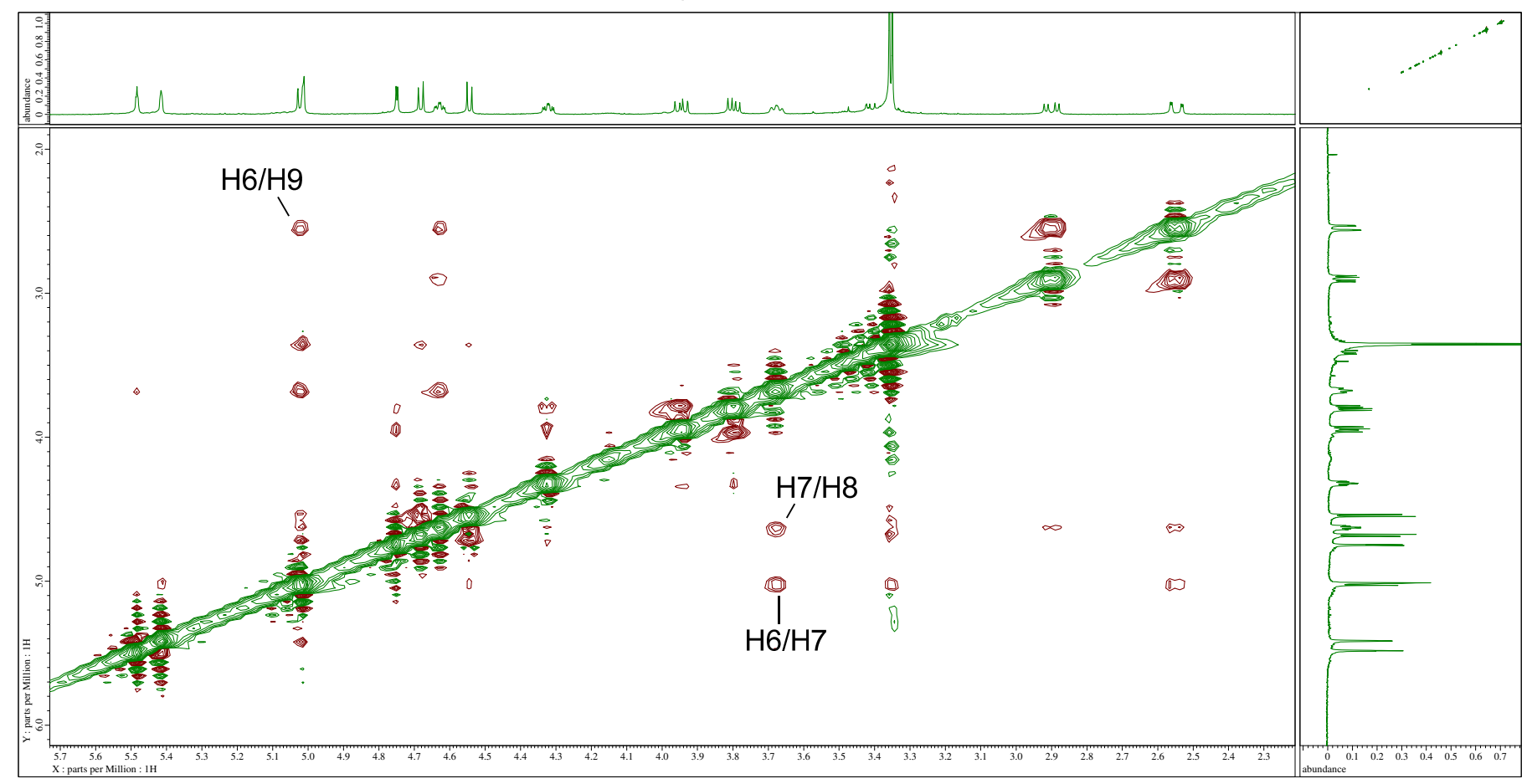




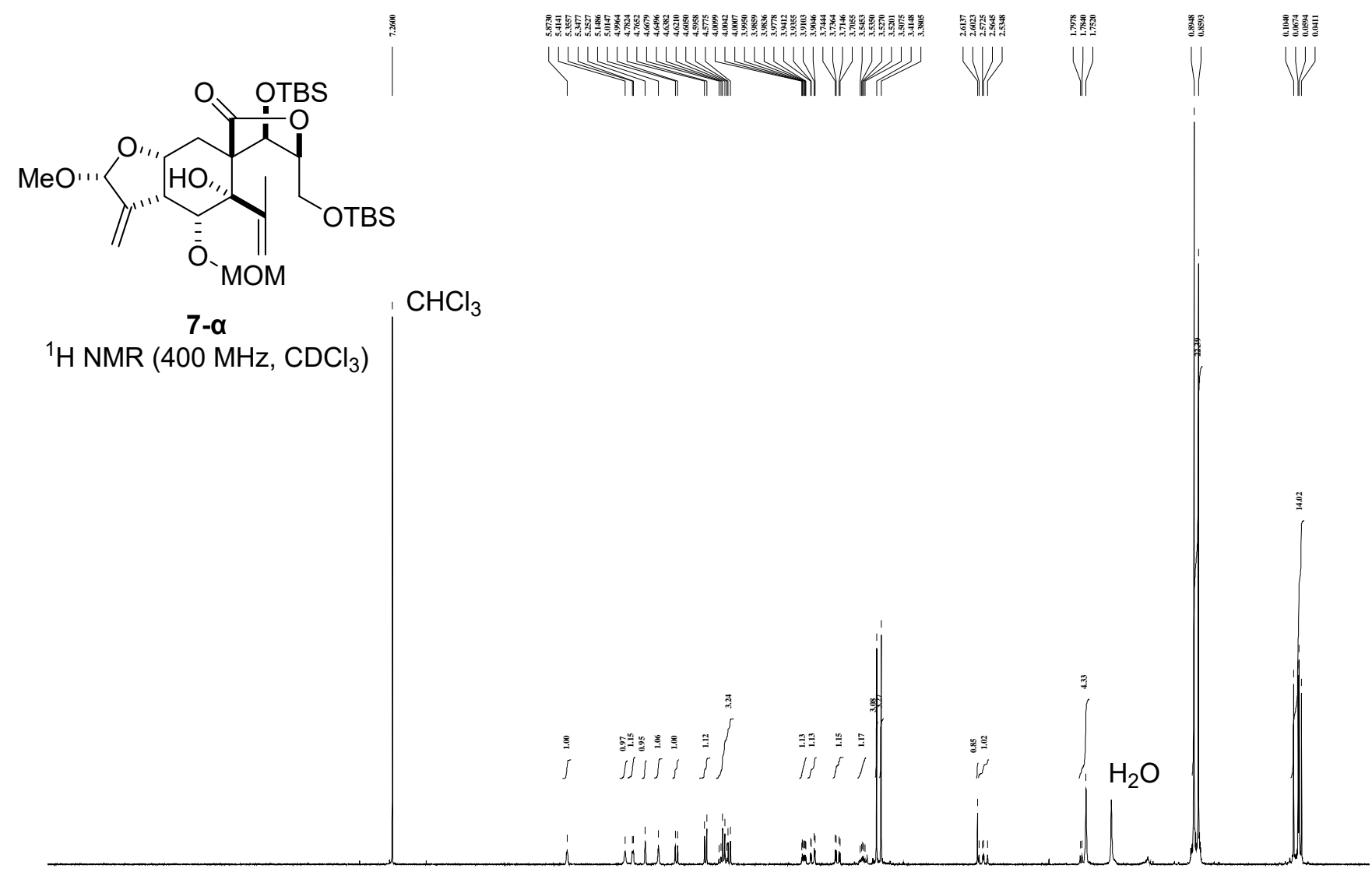

$\prod_{n=0}$

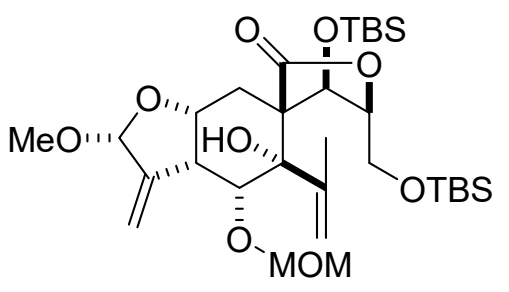

7-a

${ }^{13} \mathrm{C}\left\{{ }^{1} \mathrm{H}\right\}$ NMR $\left(100 \mathrm{MHz}, \mathrm{CDCl}_{3}\right)$

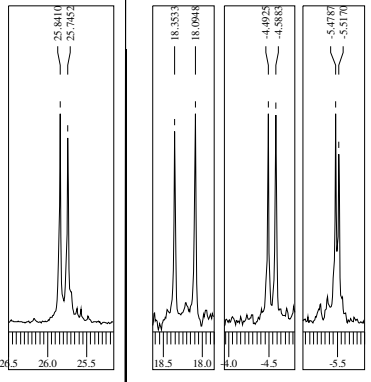

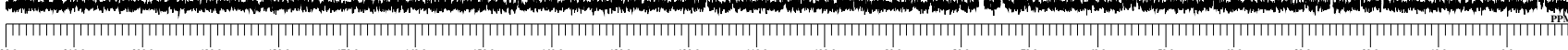

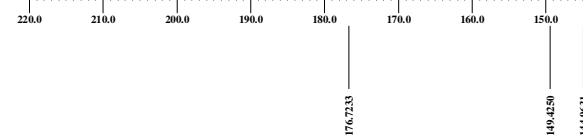




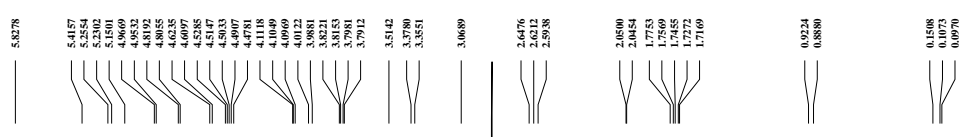

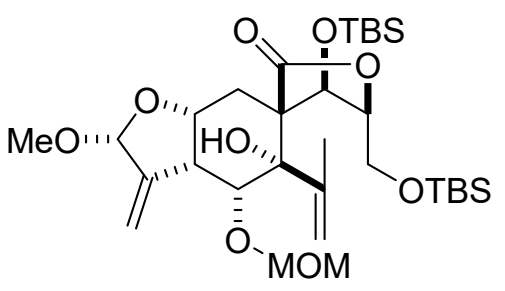

7- $\alpha$

${ }^{1} \mathrm{H}$ NMR $\left(500 \mathrm{MHz}, \mathrm{CO}\left(\mathrm{CD}_{3}\right)_{2}\right)$
$\mathrm{H}_{2} \mathrm{O}$

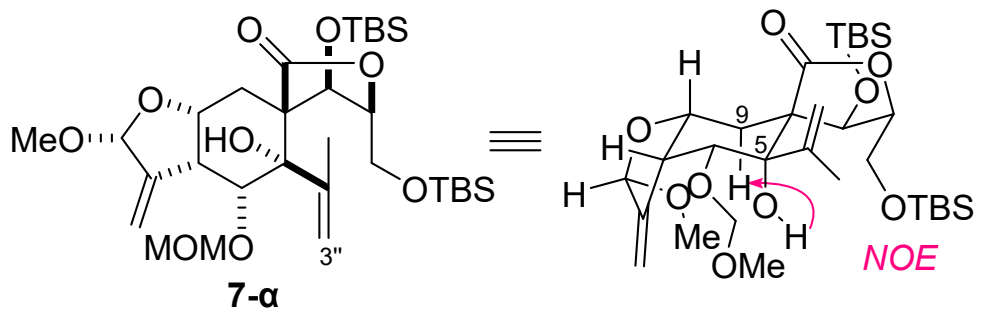

\section{7-a}
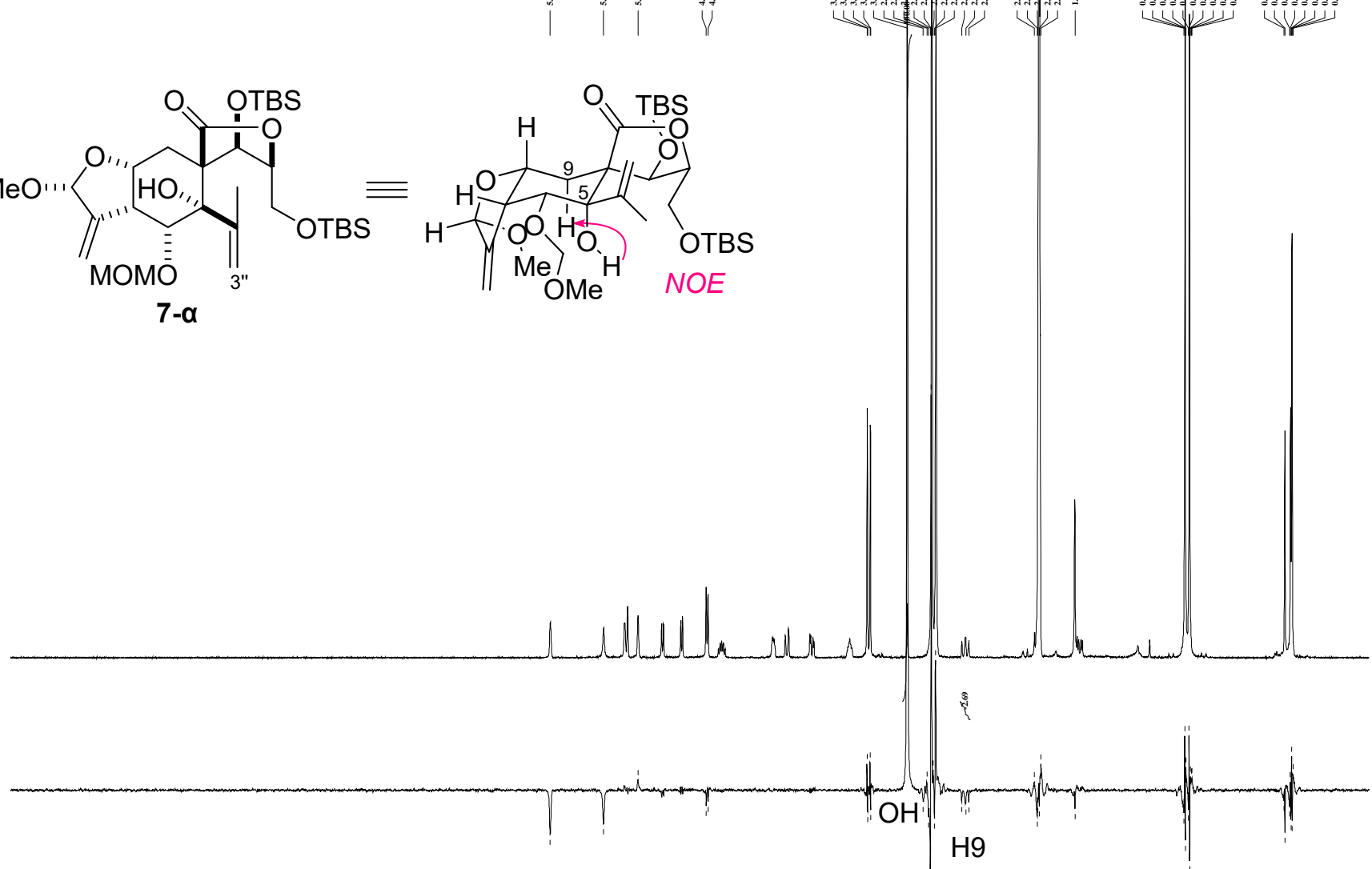


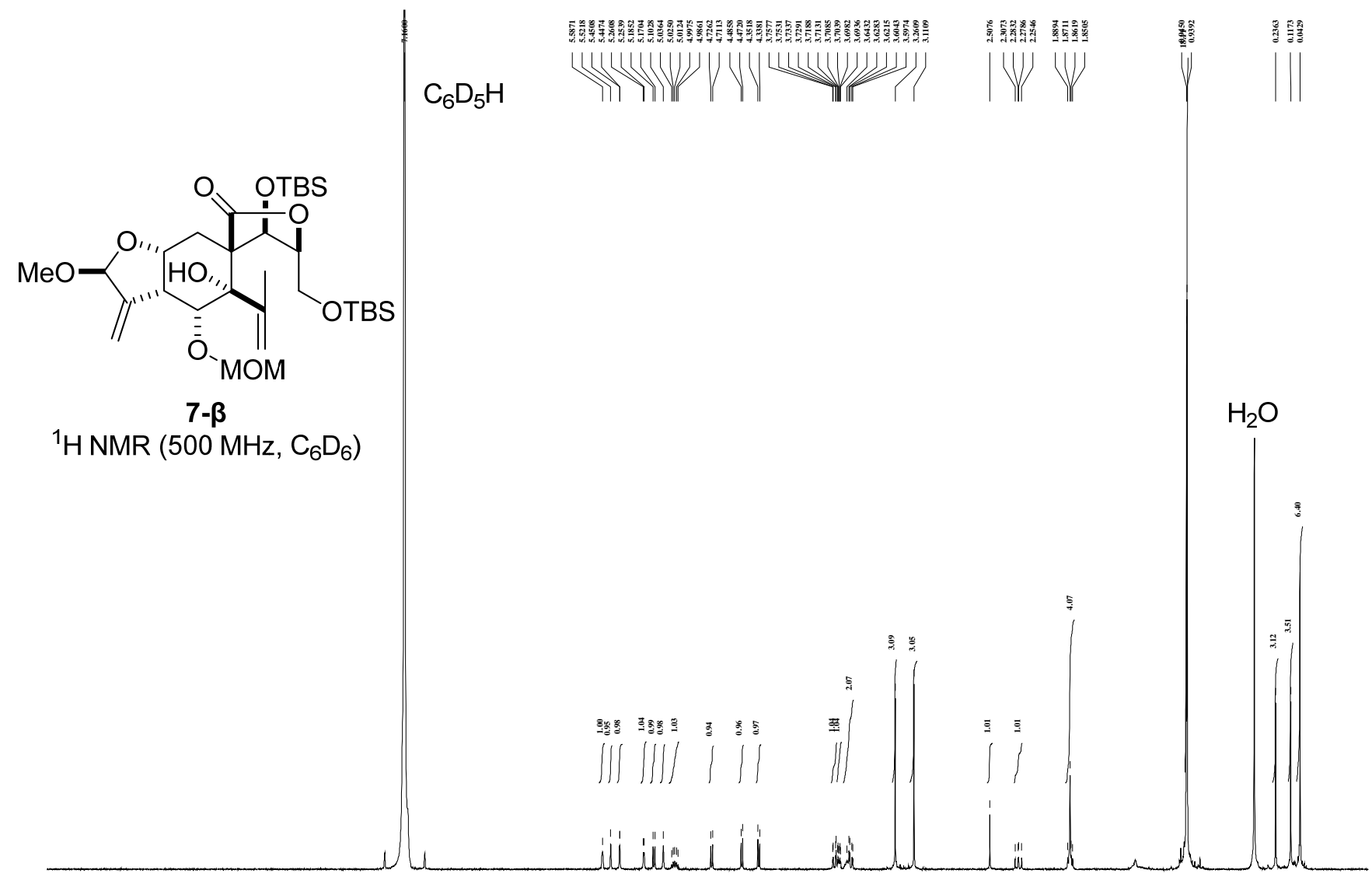

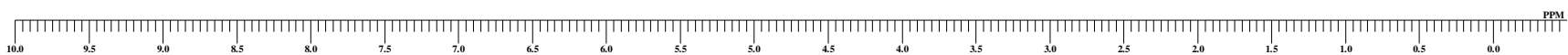

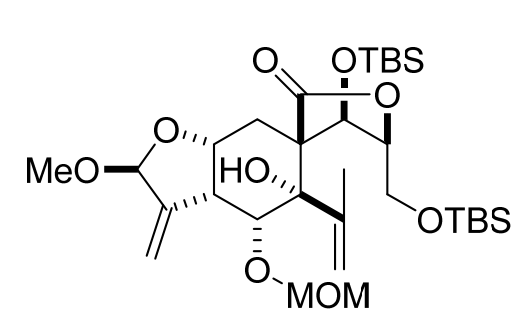

7- $\beta$

${ }^{13} \mathrm{C}\left\{{ }^{1} \mathrm{H}\right\} \operatorname{NMR}\left(100 \mathrm{MHz}, \mathrm{C}_{6} \mathrm{D}_{6}\right)$

\section{$\mathrm{C}_{6} \mathrm{D}_{6}$}

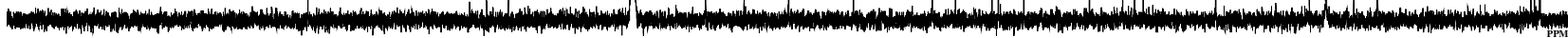

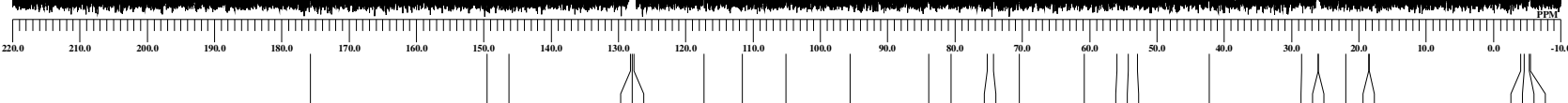




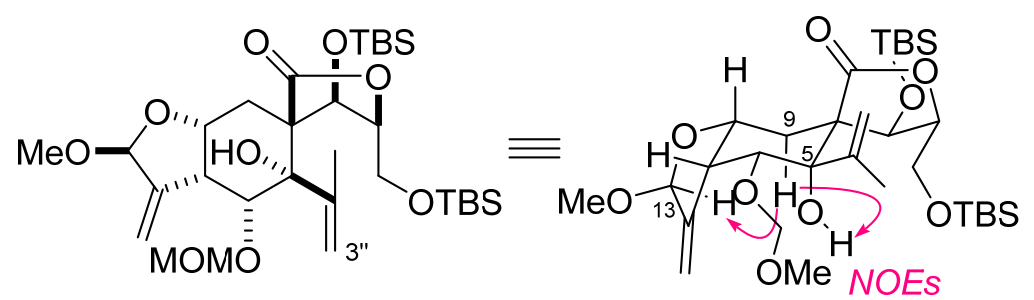

7- $\beta$

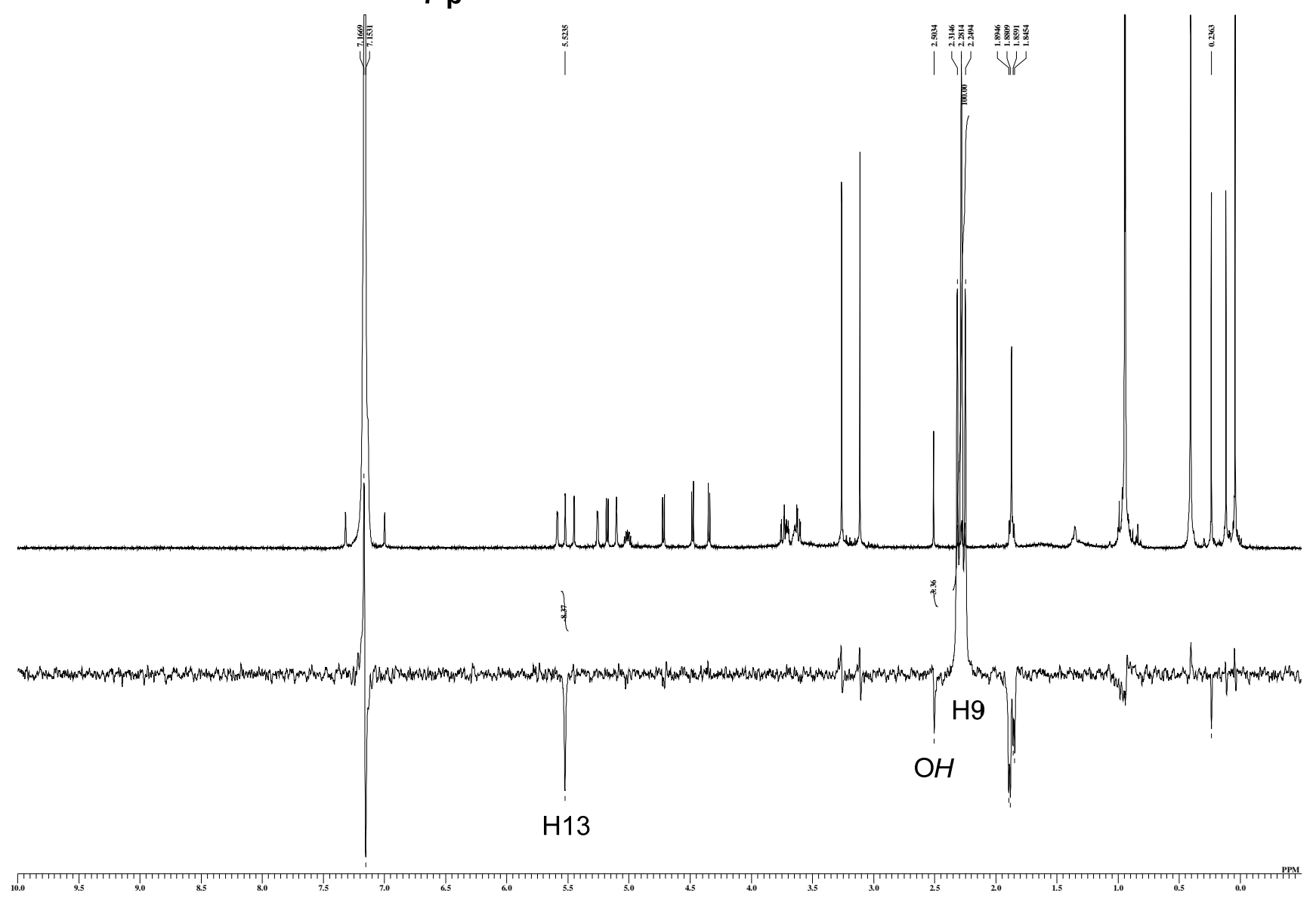




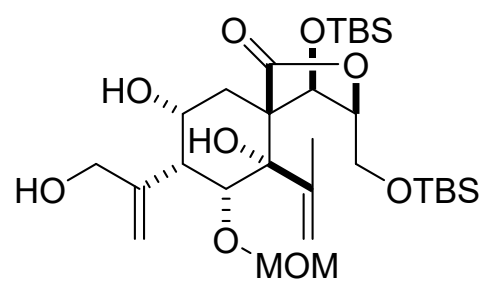

26

${ }^{1} \mathrm{H}$ NMR $\left(400 \mathrm{MHz}, \mathrm{C}_{6} \mathrm{D}_{6}\right)$
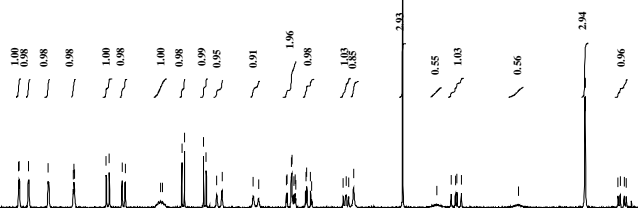

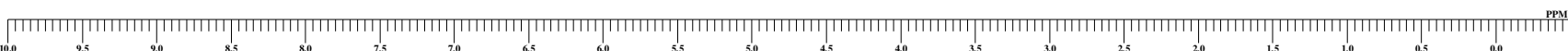

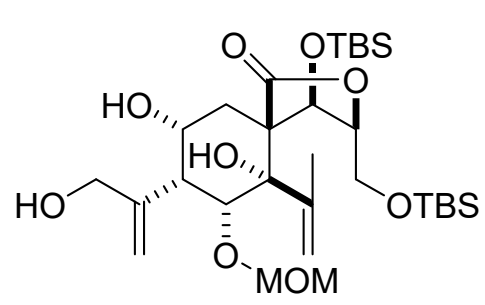

26

${ }^{13} \mathrm{C}\left\{{ }^{1} \mathrm{H}\right\} \operatorname{NMR}\left(125 \mathrm{MHz}, \mathrm{C}_{6} \mathrm{D}_{6}\right)$

$$
\mathrm{C}_{6} \mathrm{D}_{6}
$$

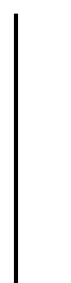




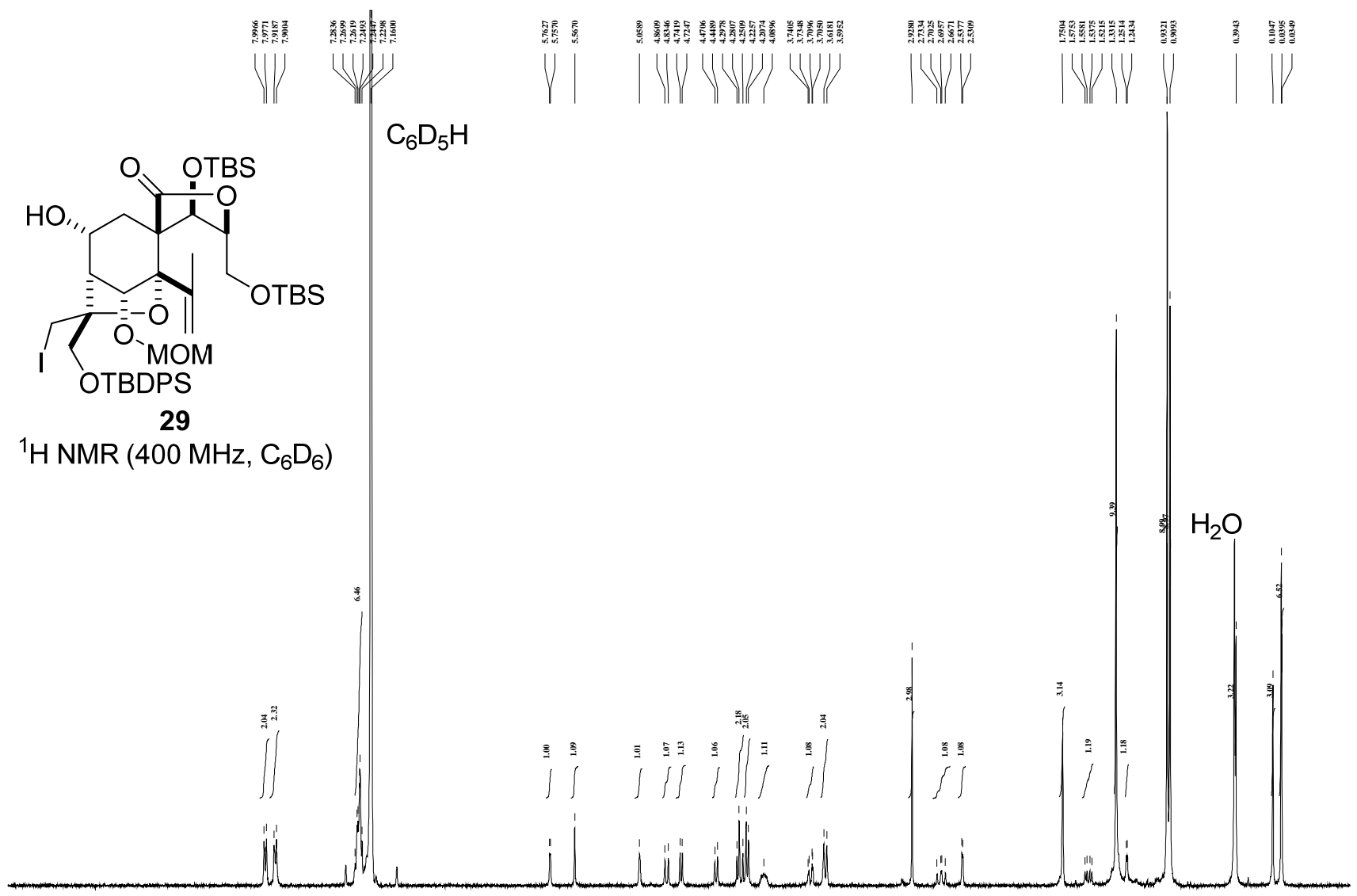

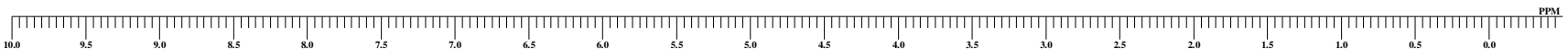

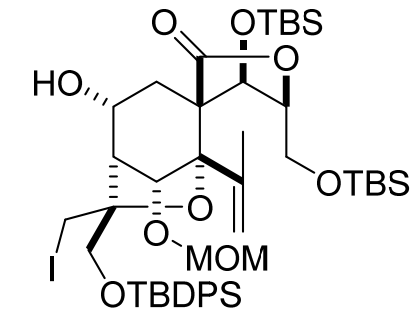

29

${ }^{13} \mathrm{C}\left\{{ }^{1} \mathrm{H}\right\} \mathrm{NMR}\left(100 \mathrm{MHz}, \mathrm{C}_{6} \mathrm{D}_{6}\right)$

\section{$\mathrm{C}_{6} \mathrm{D}_{6}$}
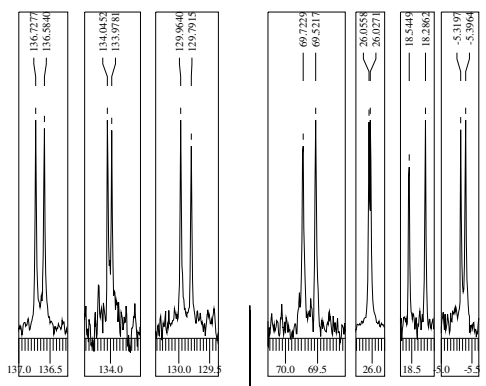

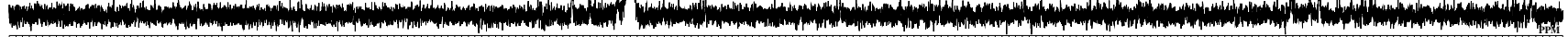

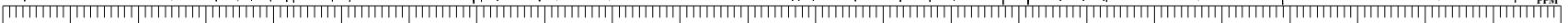

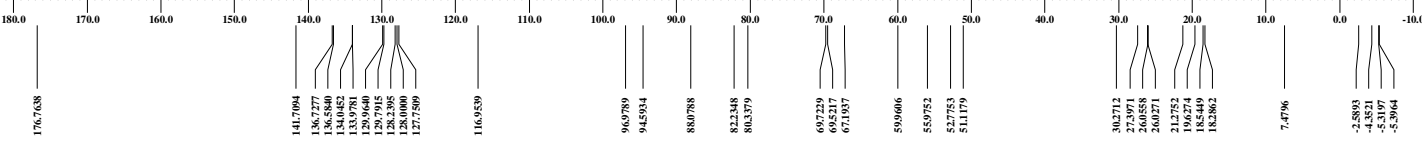




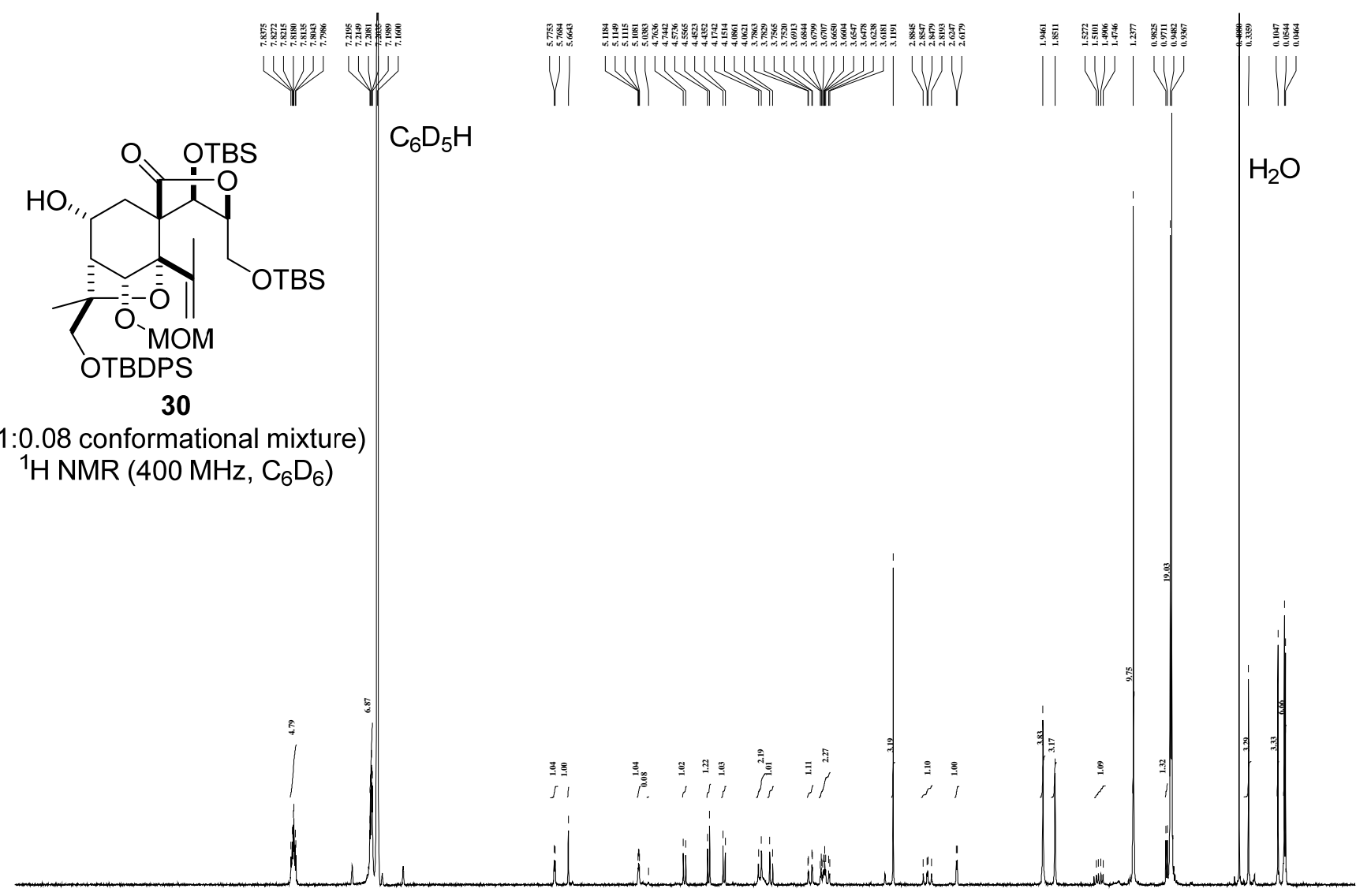

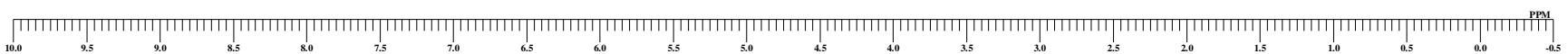

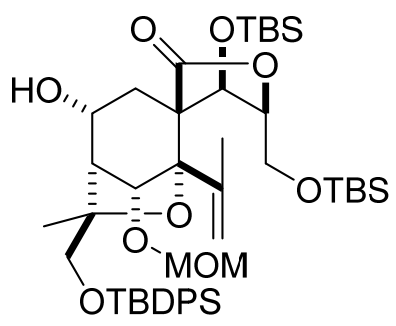

30

$(1: 0.08$ conformational mixture) ${ }^{13} \mathrm{C}\left\{{ }^{1} \mathrm{H}\right\} \mathrm{NMR}\left(100 \mathrm{MHz}, \mathrm{C}_{6} \mathrm{D}_{6}\right)$
$\mathrm{C}_{6} \mathrm{D}_{6}$

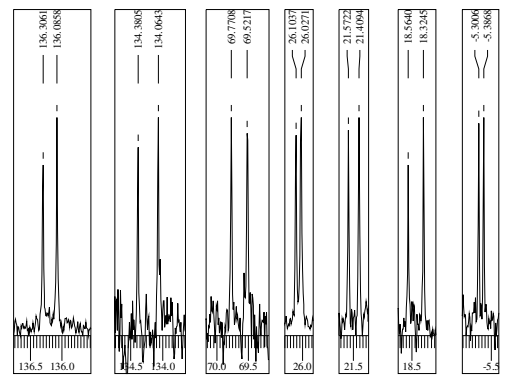



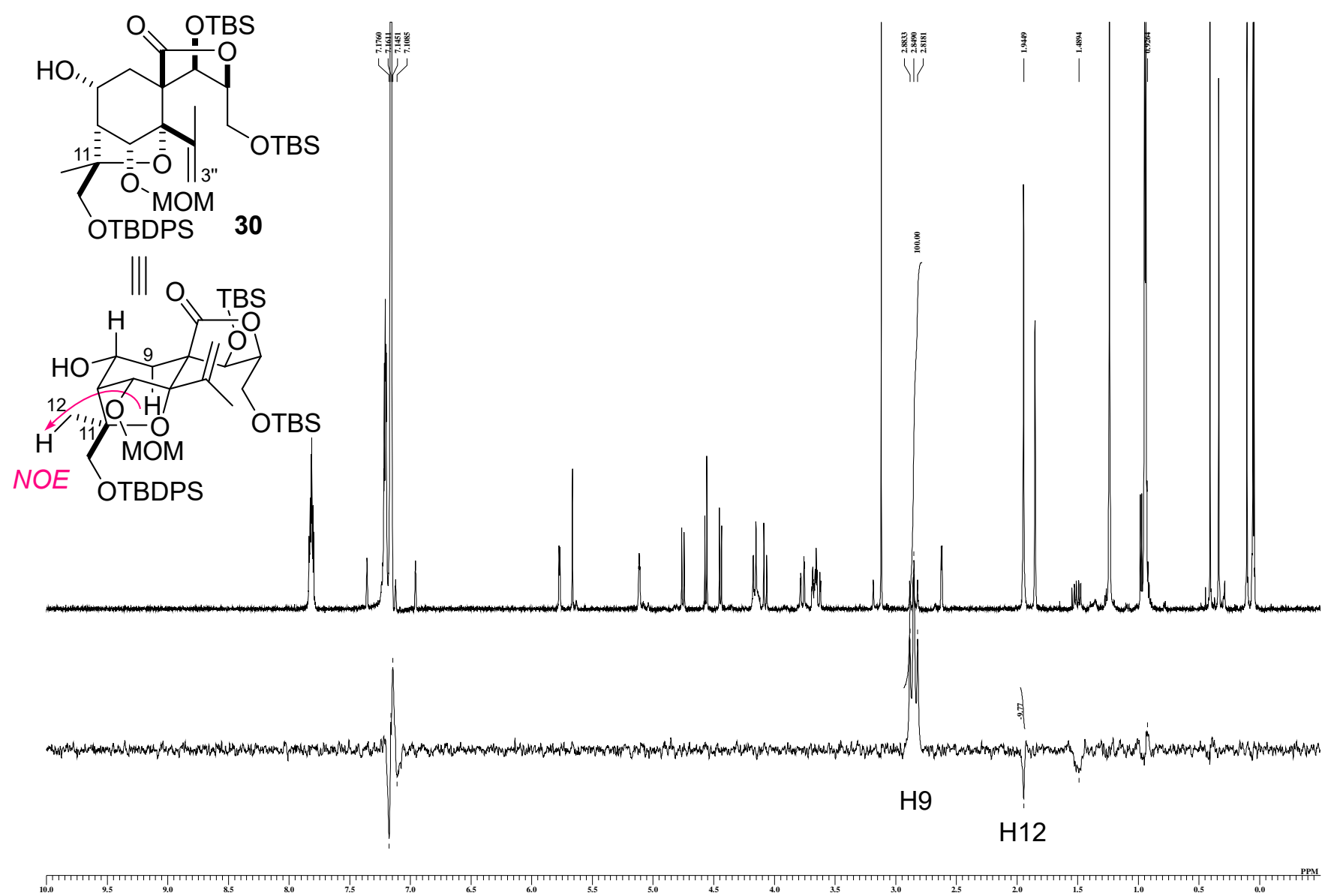

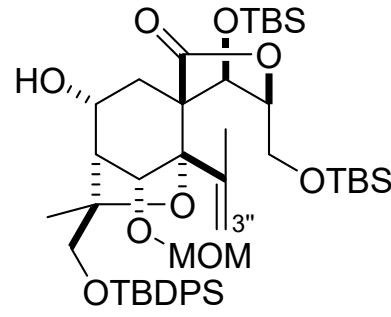

30

(1:0.08 conformational mixture)
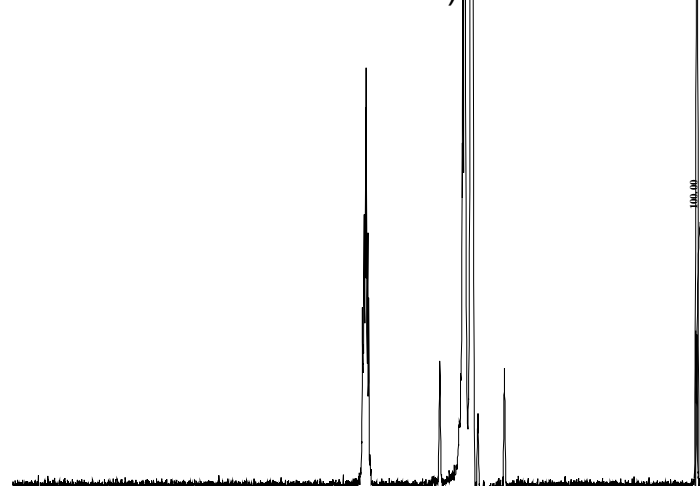

$\mathrm{H} 3$ " of the minor conformer

irradiation of $\mathrm{H} 3 "$

of the major

conformer 


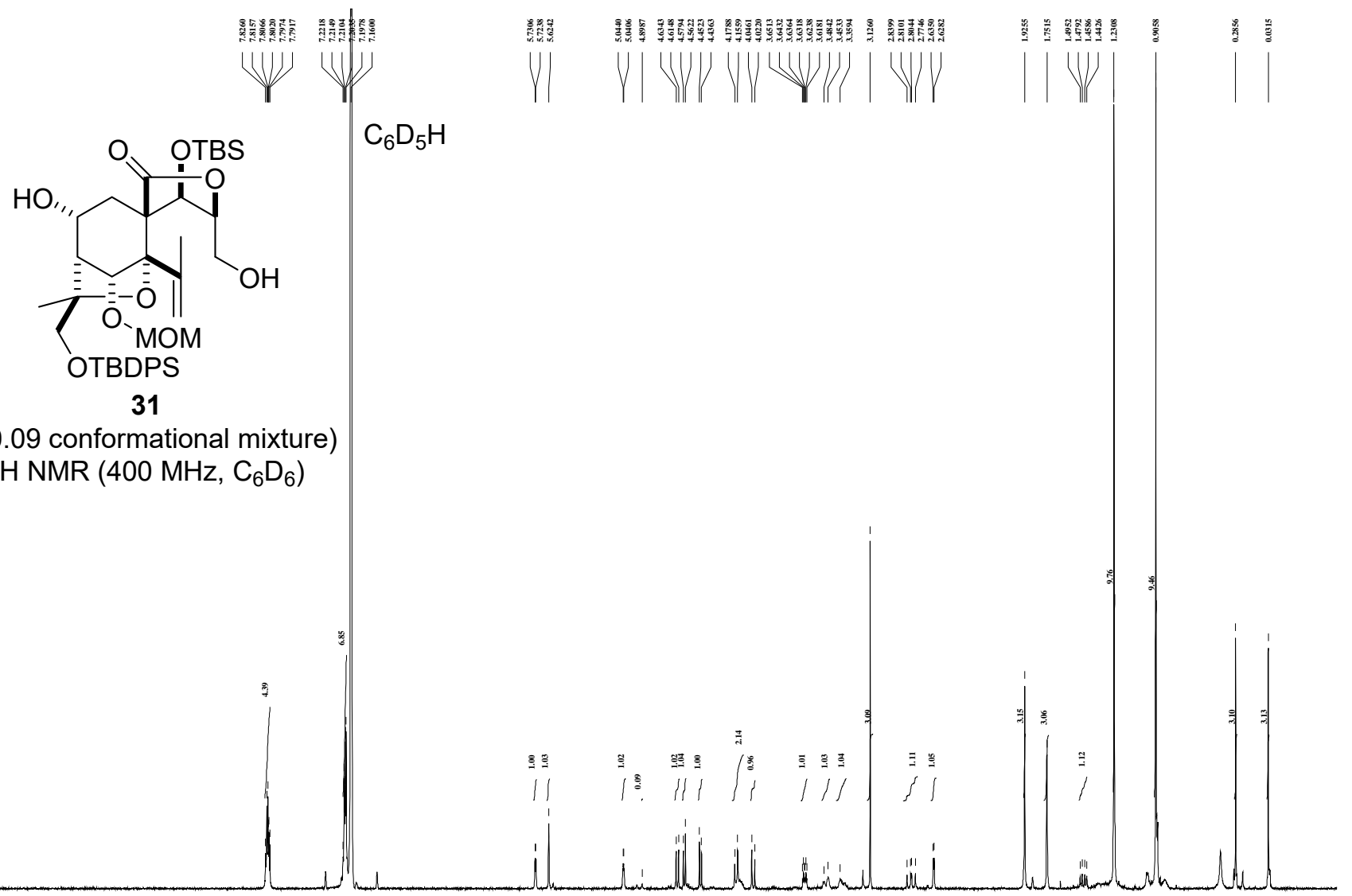

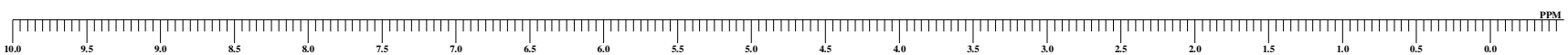

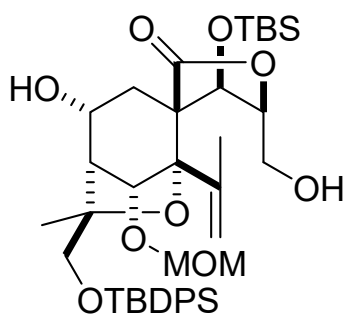

31

(1:0.09 conformational mixture) ${ }^{13} \mathrm{C}\left\{{ }^{1} \mathrm{H}\right\}$ NMR $\left(125 \mathrm{MHz}, \mathrm{C}_{6} \mathrm{D}_{6}\right)$

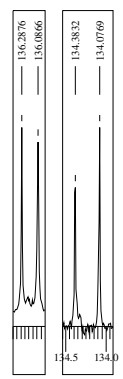

$\mathrm{C}_{6} \mathrm{D}_{6}$

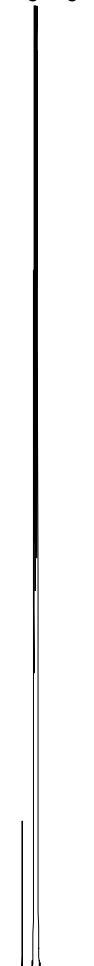

$\prod_{220.0}$ 


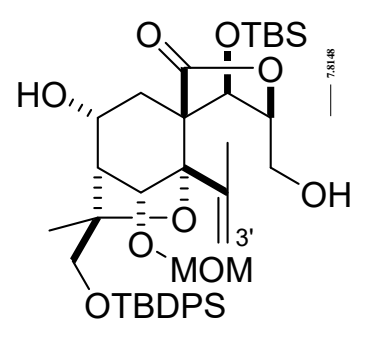

31

(1:0.09 conformational mixture)

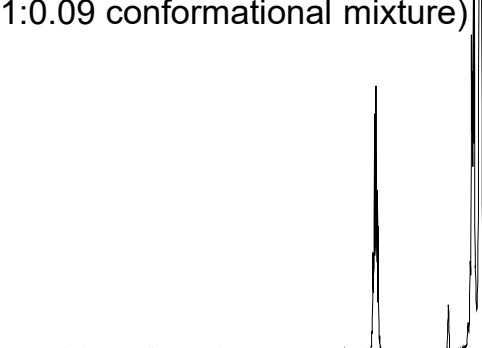

约㩆

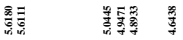

|V |||

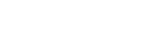

(20)

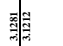

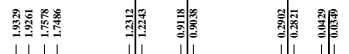<smiles>C1CCCCC1</smiles><smiles>C1CCC1</smiles>

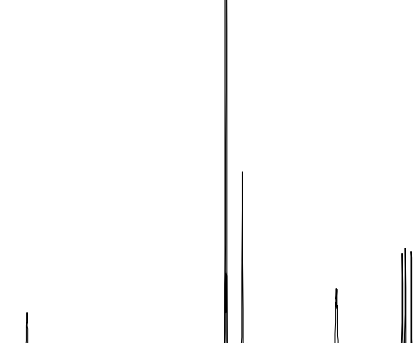

$\frac{8}{8}$

$\mathrm{H} 3$ " of the minor conformer

irradiation of $\mathrm{H} 3$ "

of the major

conformer
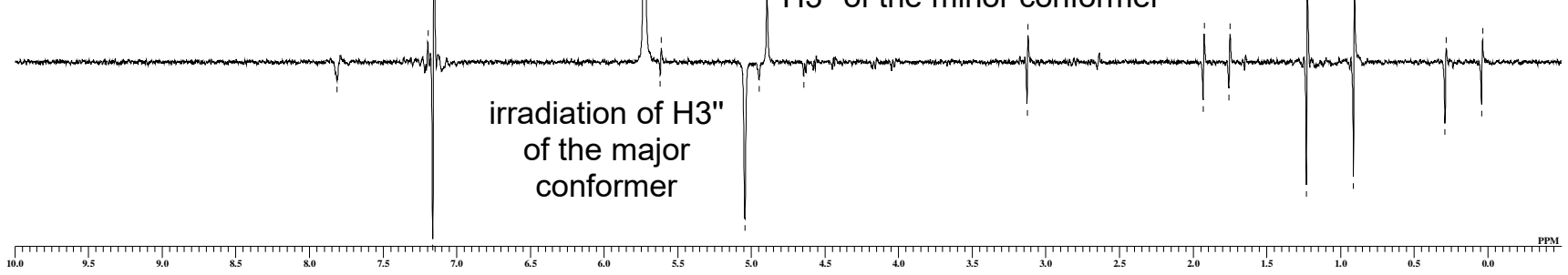


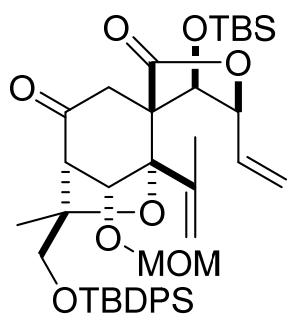

33

${ }^{1} \mathrm{H}$ NMR $\left(400 \mathrm{MHz}, \mathrm{C}_{6} \mathrm{D}_{6}\right)$

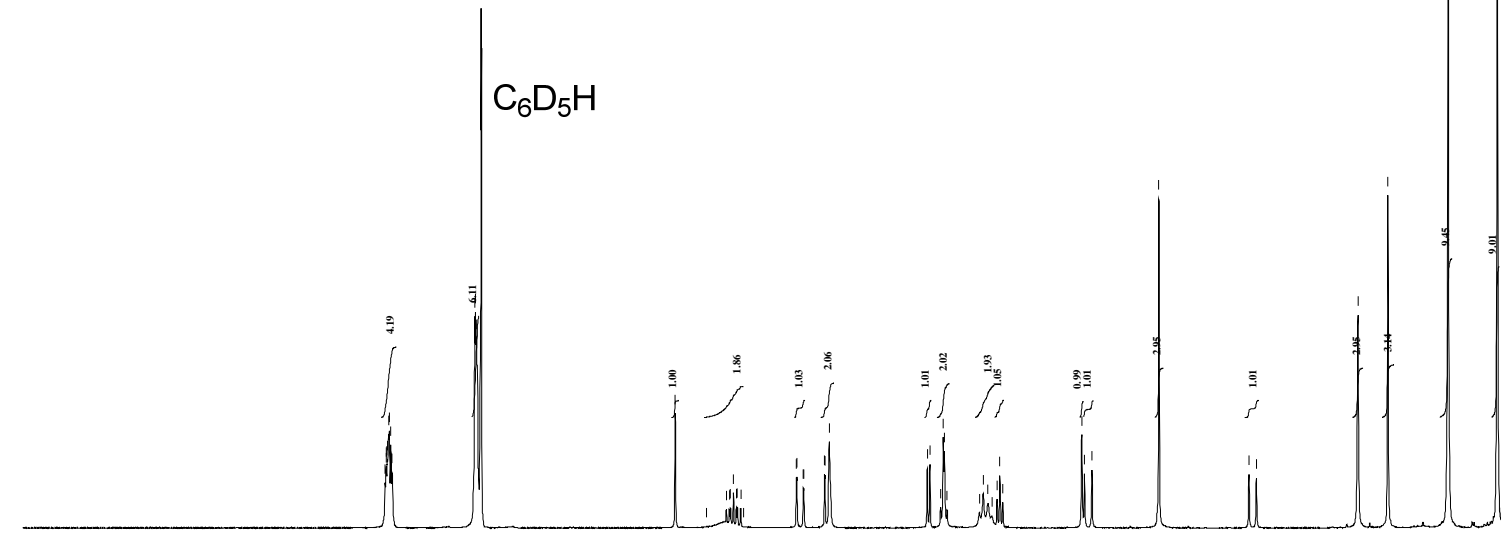

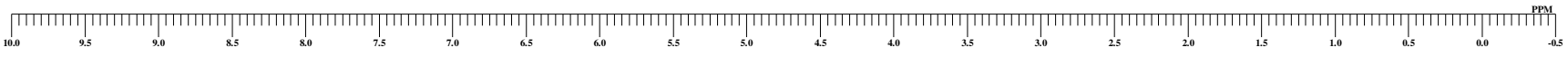

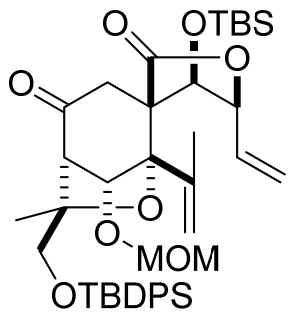

33

${ }^{13} \mathrm{C}\left\{{ }^{1} \mathrm{H}\right\} \mathrm{NMR}\left(125 \mathrm{MHz}, \mathrm{C}_{6} \mathrm{D}_{6}\right)$
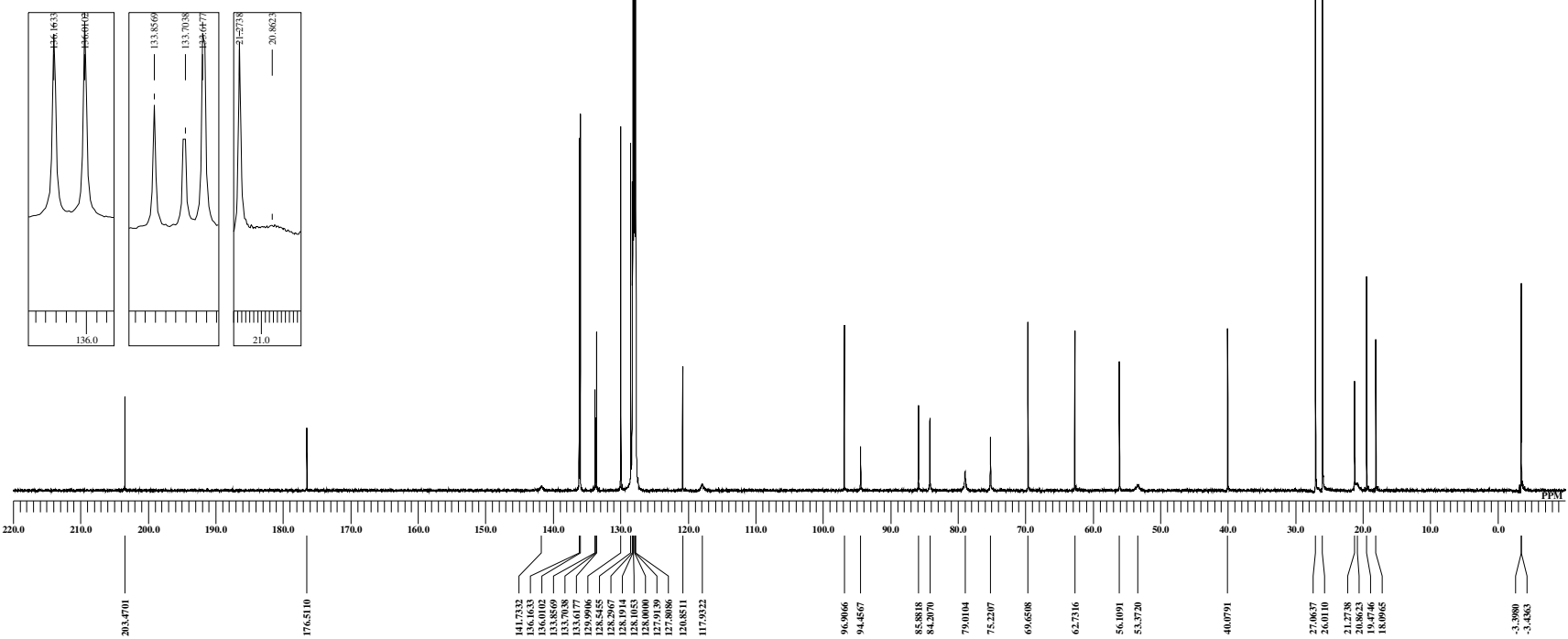


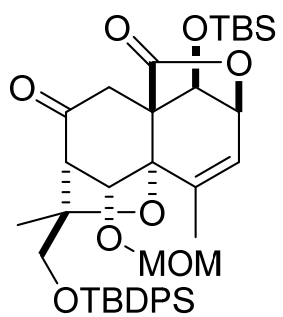

6

${ }^{1} \mathrm{H}$ NMR $\left(500 \mathrm{MHz}, \mathrm{C}_{6} \mathrm{D}_{6}\right)$

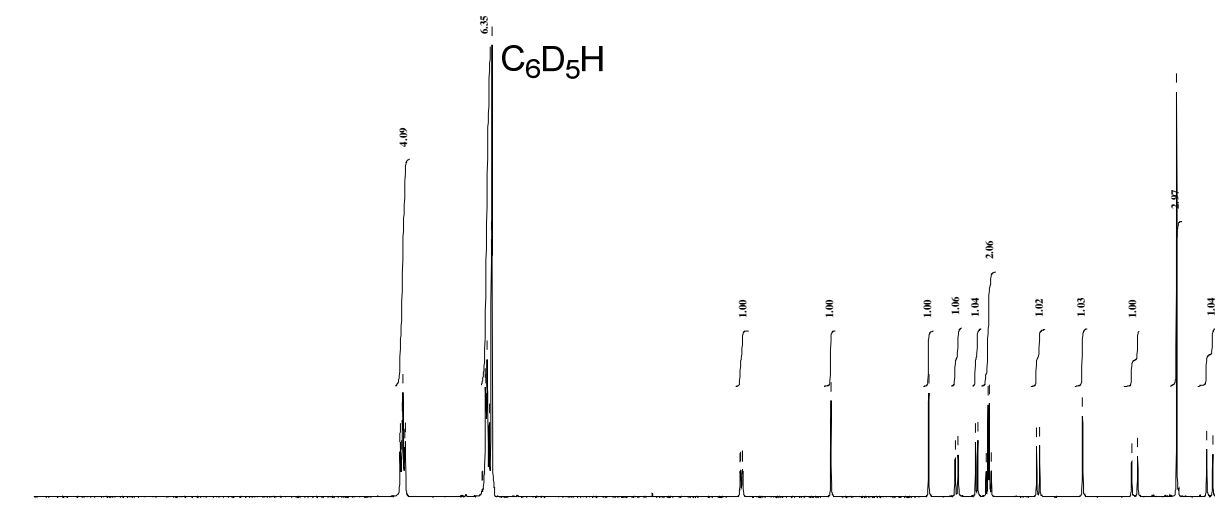

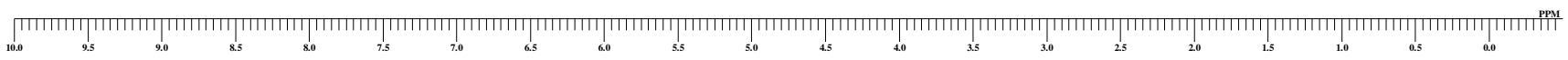

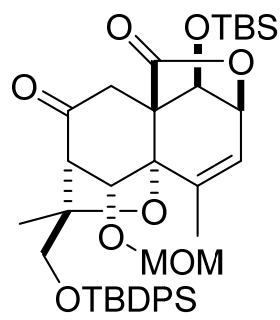

6

${ }^{13} \mathrm{C}\left\{{ }^{1} \mathrm{H}\right\} \mathrm{NMR}\left(125 \mathrm{MHz}, \mathrm{C}_{6} \mathrm{D}_{6}\right)$
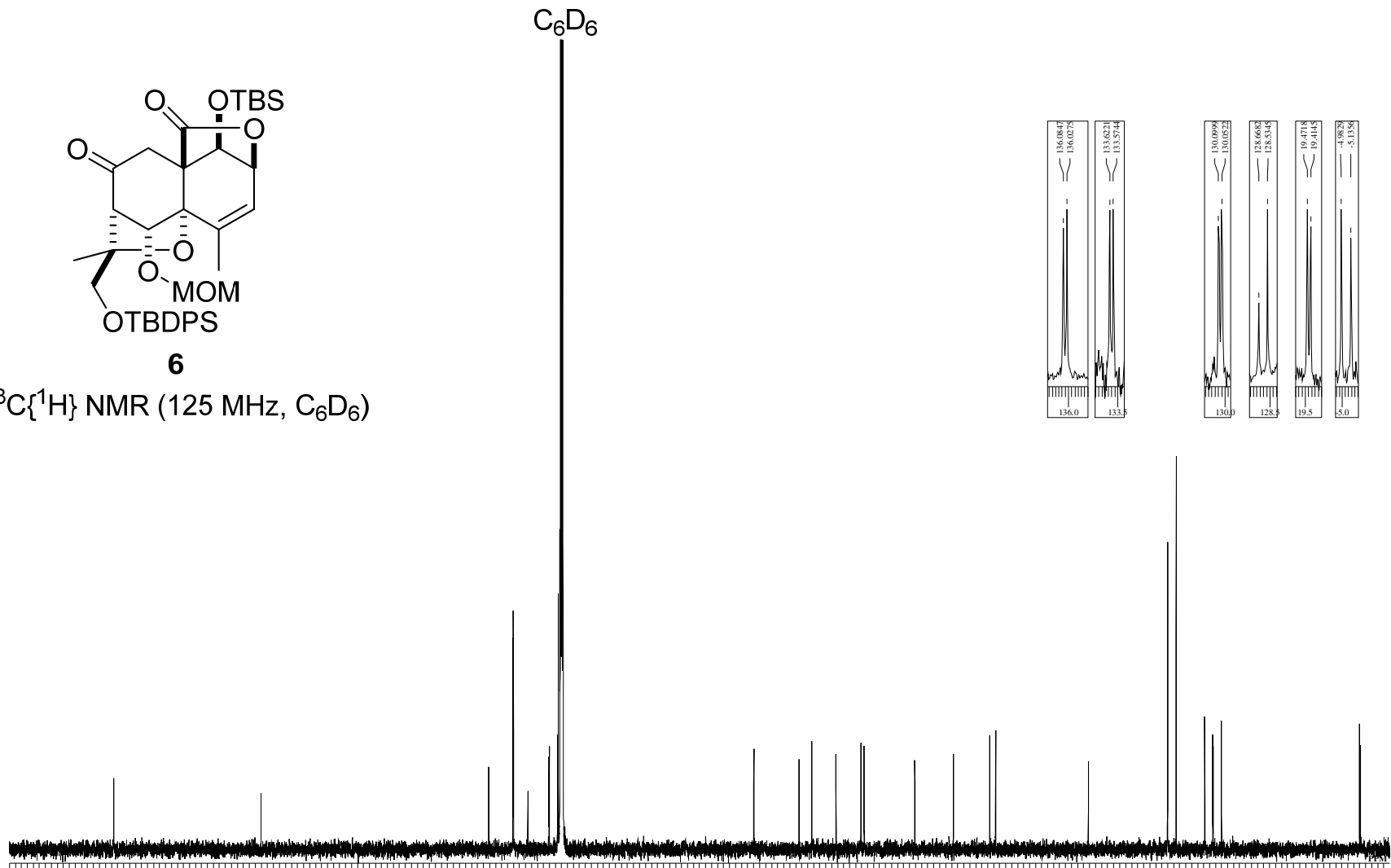

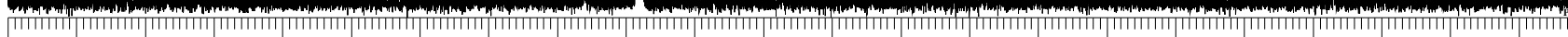

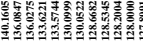

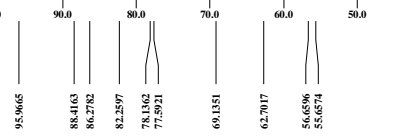

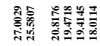




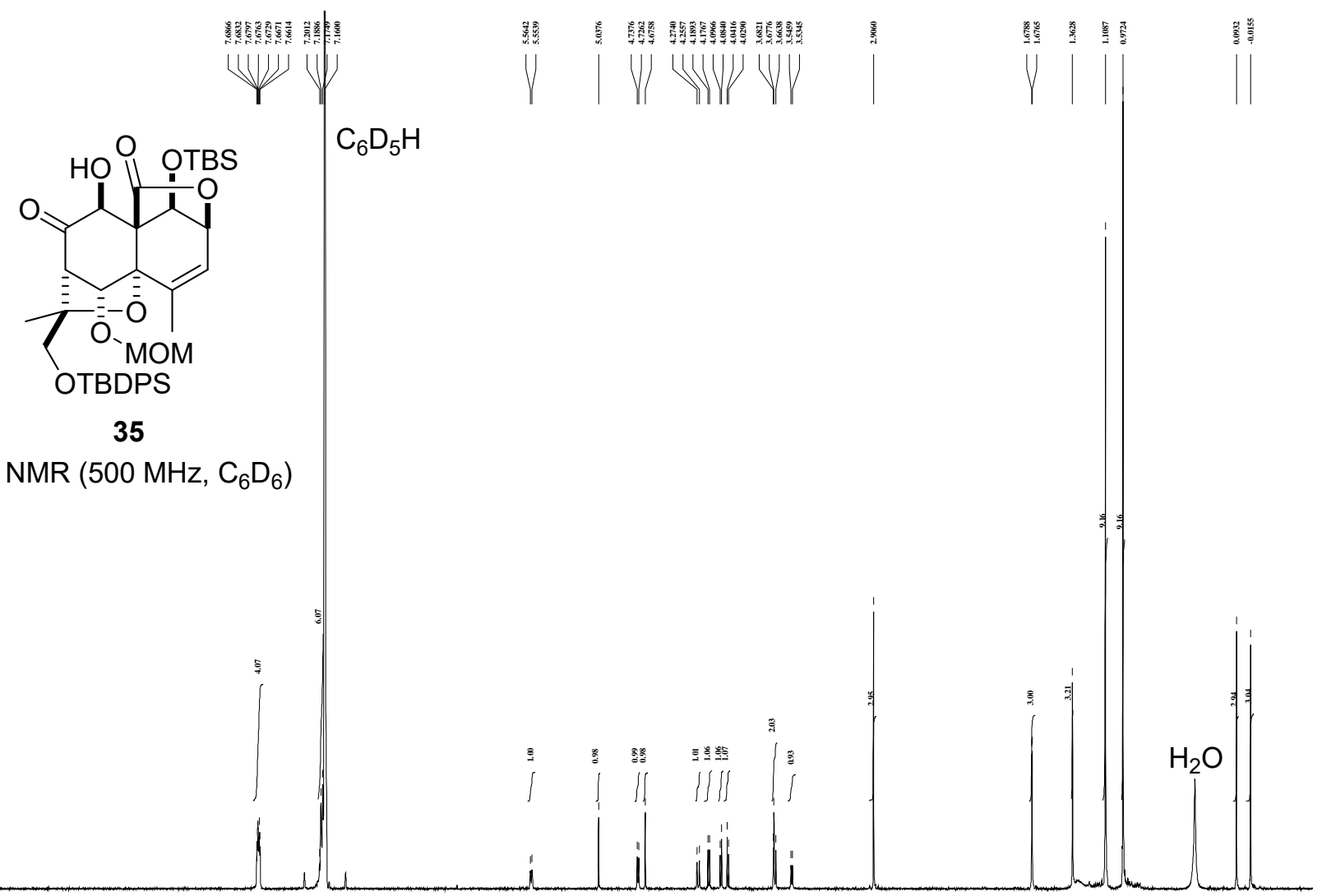

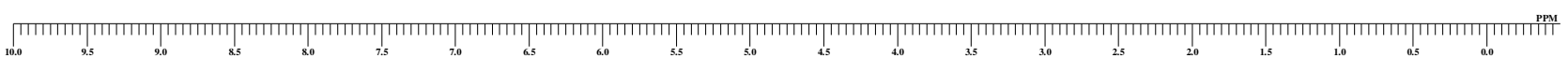

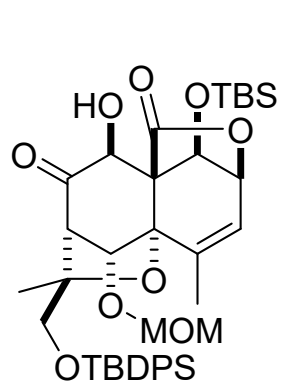

35

${ }^{13} \mathrm{C}\left\{{ }^{1} \mathrm{H}\right\}$ NMR $\left(125 \mathrm{MHz}, \mathrm{C}_{6} \mathrm{D}_{6}\right)$

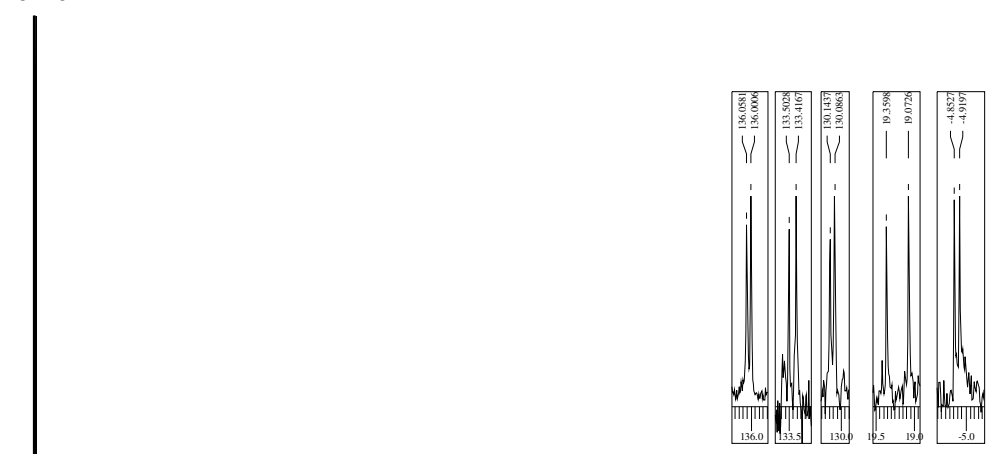

$$
\mathrm{C}_{6} \mathrm{D}_{6}
$$

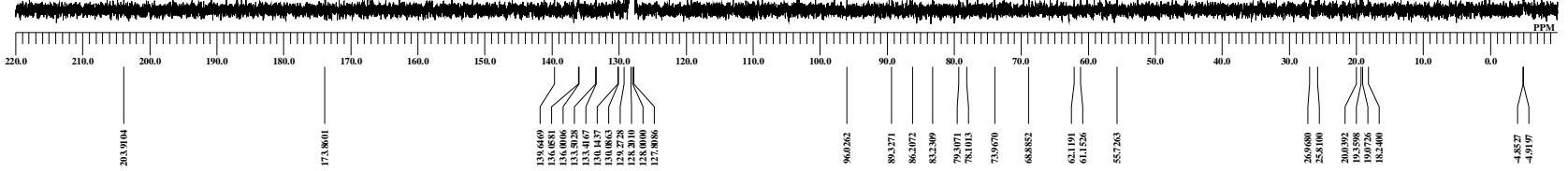


Supporting Information

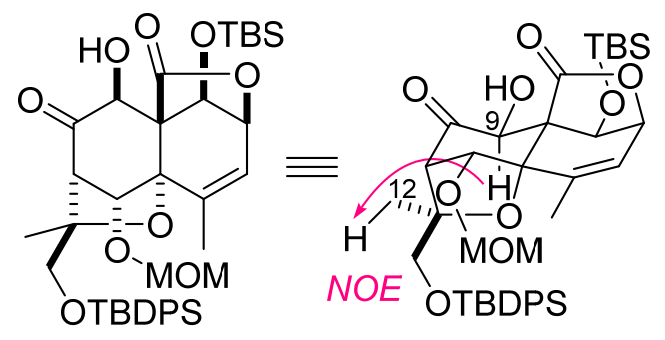

35

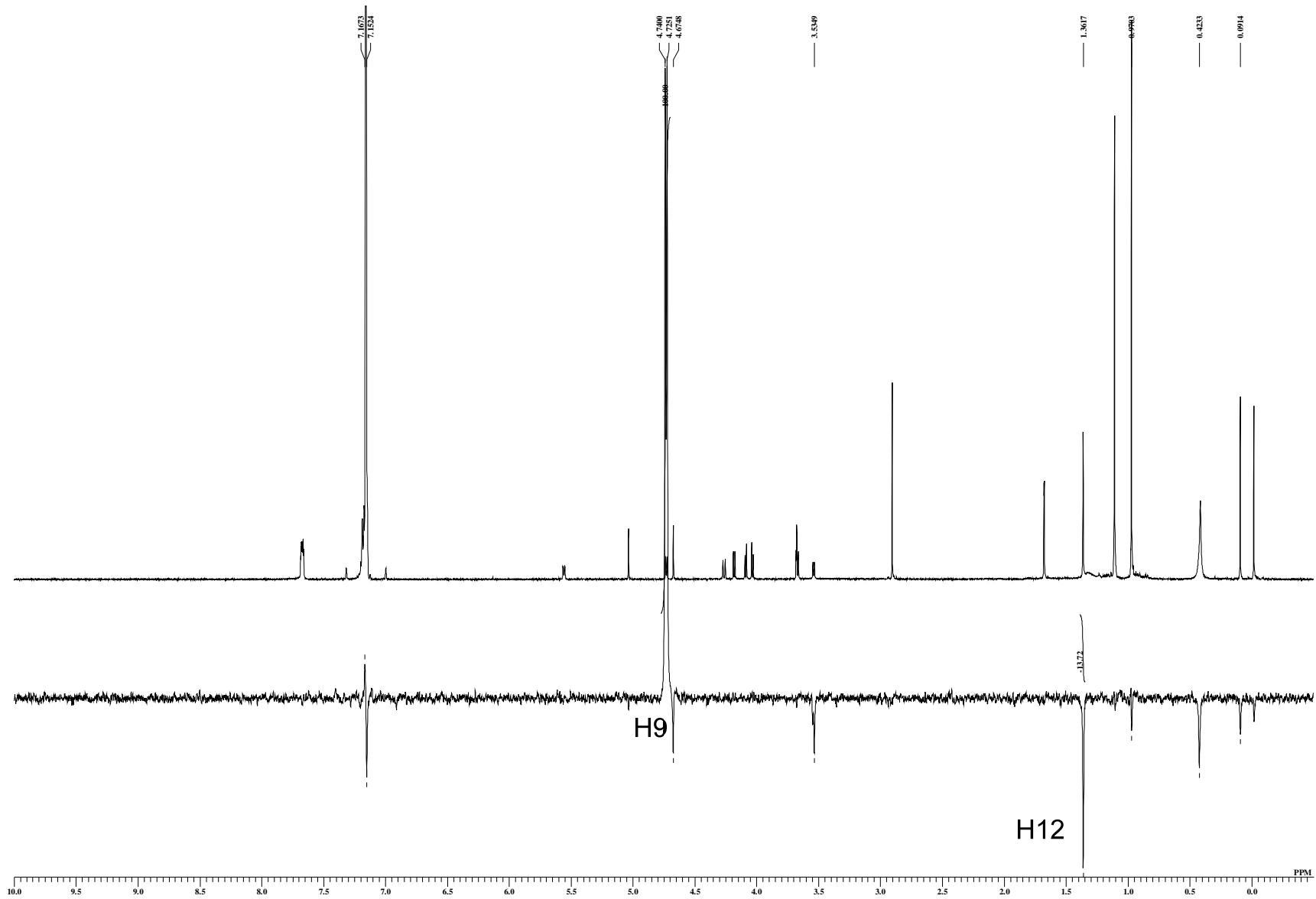




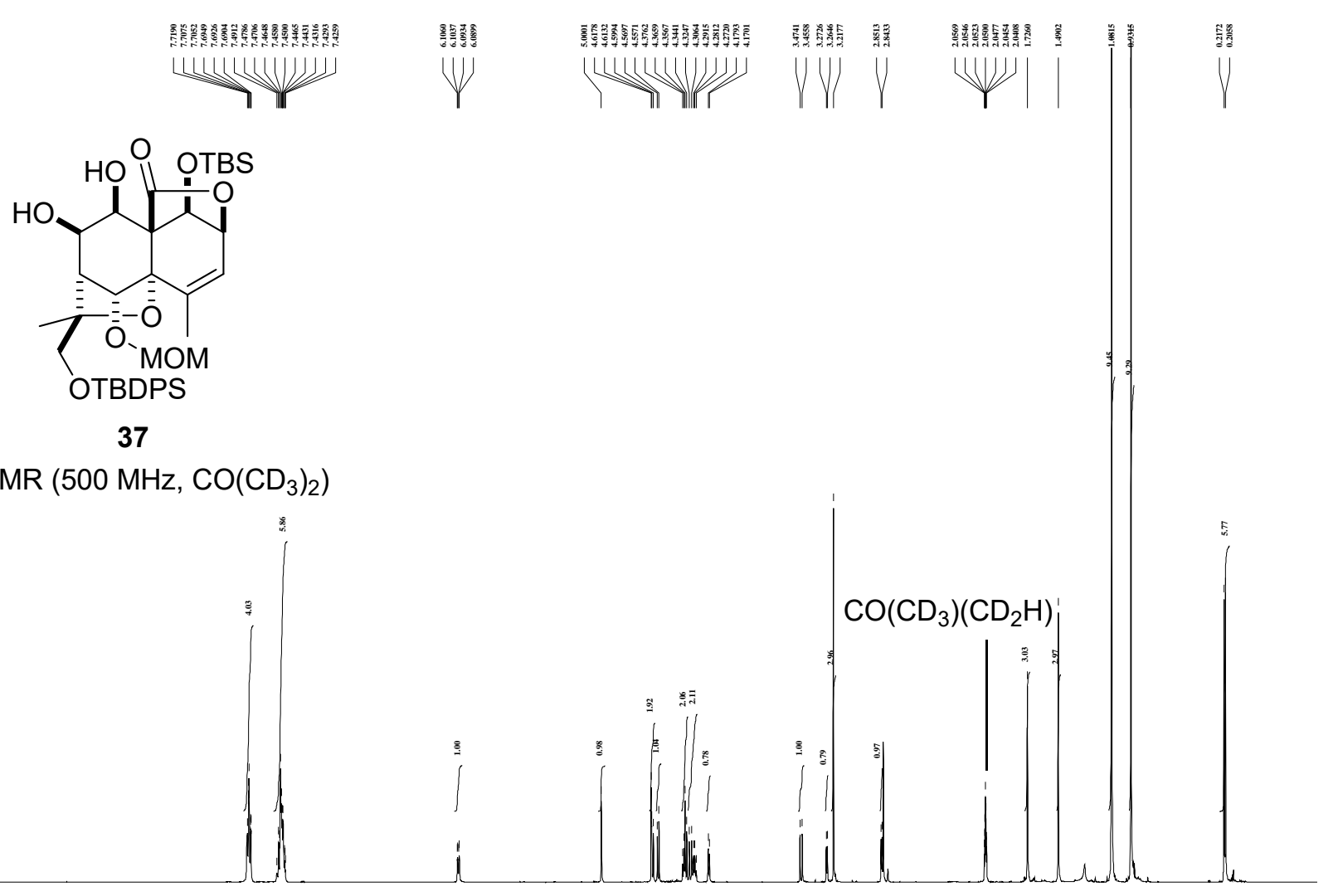

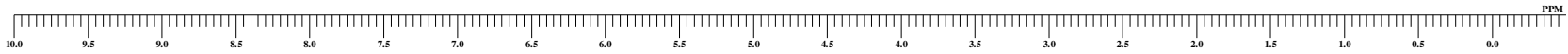

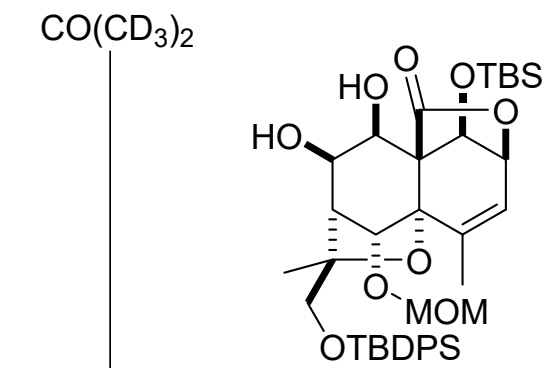

37

${ }^{13} \mathrm{C}\left\{{ }^{1} \mathrm{H}\right\}$ NMR $\left(125 \mathrm{MHz}, \mathrm{CO}\left(\mathrm{CD}_{3}\right)_{2}\right)$

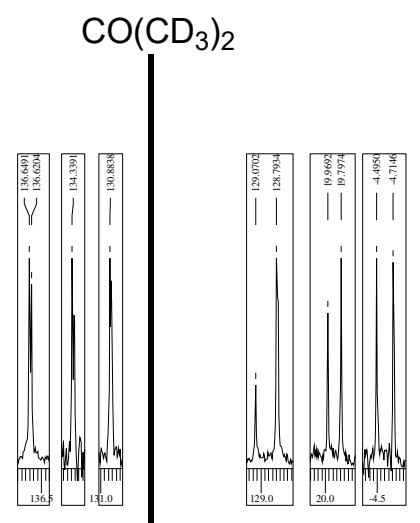

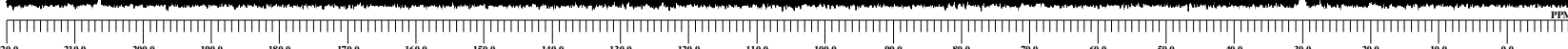

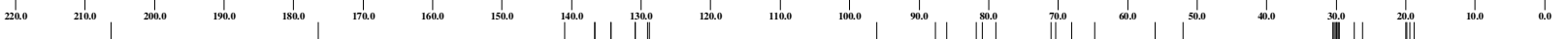




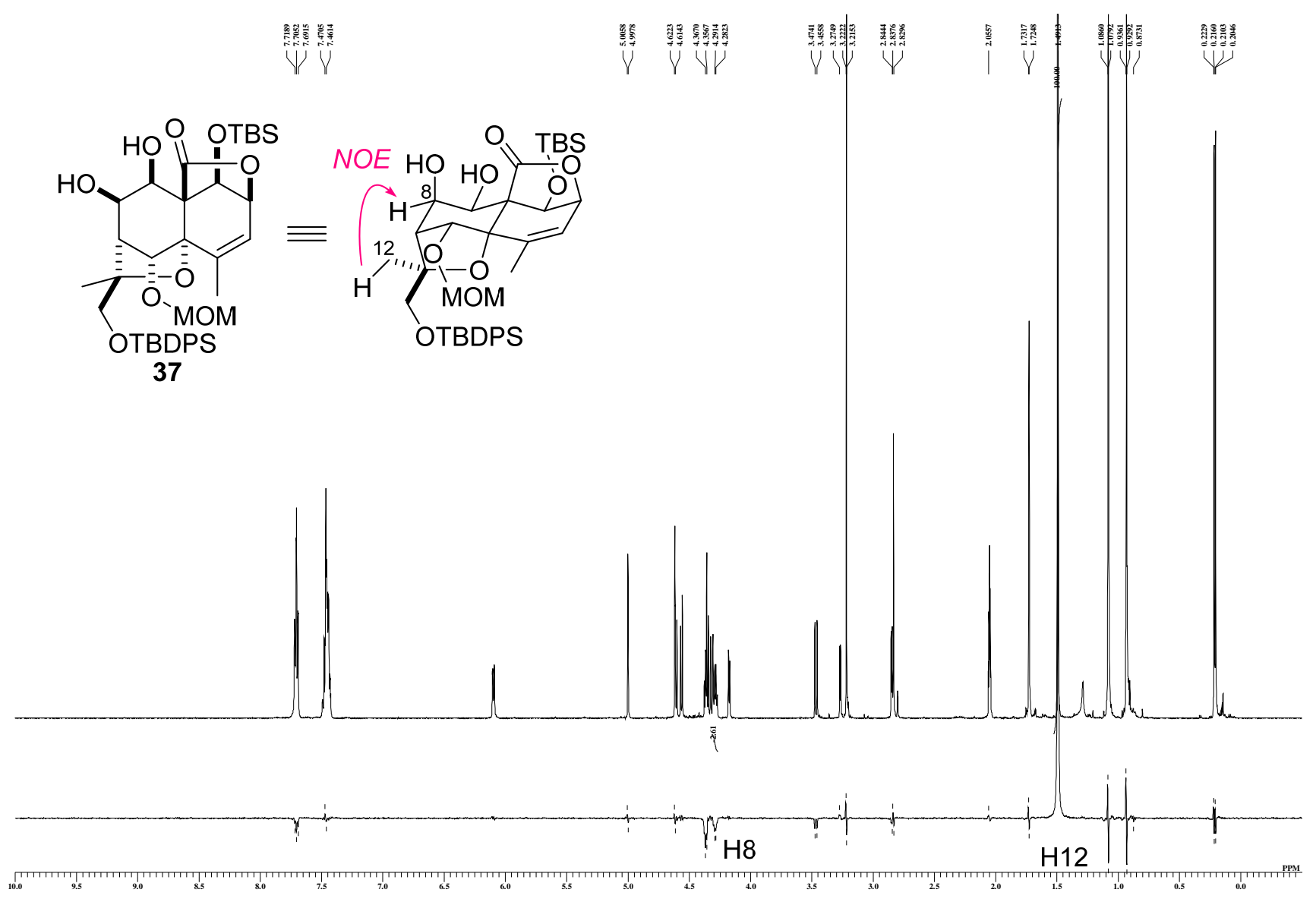




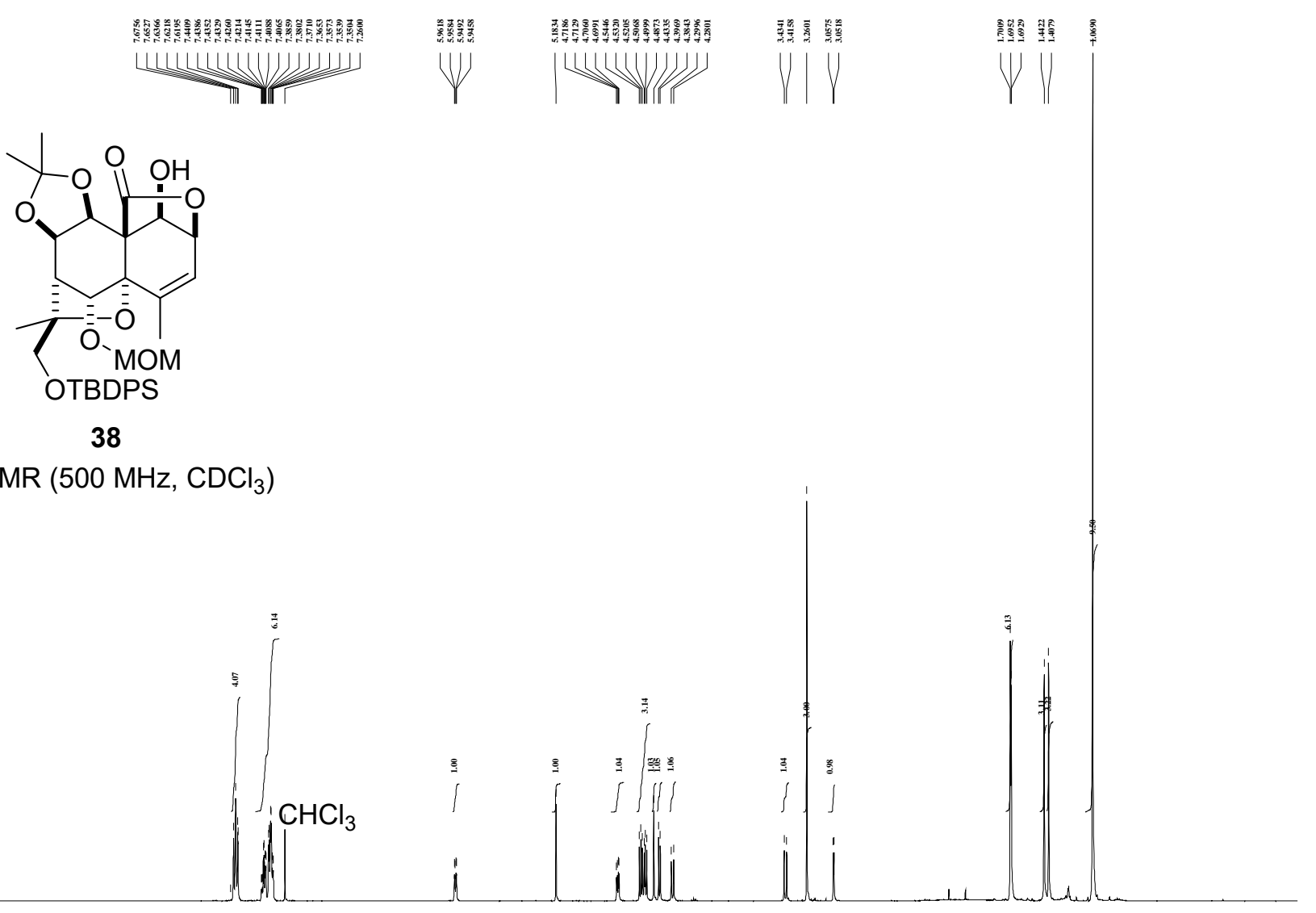

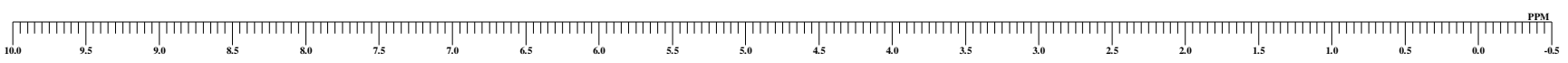

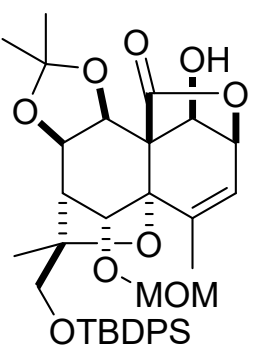

38

${ }^{13} \mathrm{C}\left\{{ }^{1} \mathrm{H}\right\}$ NMR $\left(125 \mathrm{MHz}, \mathrm{CDCl}_{3}\right)$
$\mathrm{CDCl}_{3}$

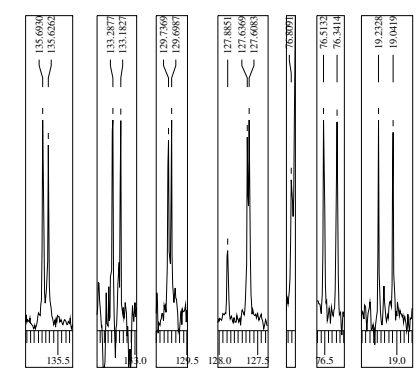




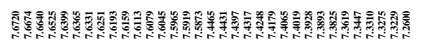

(n)

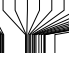

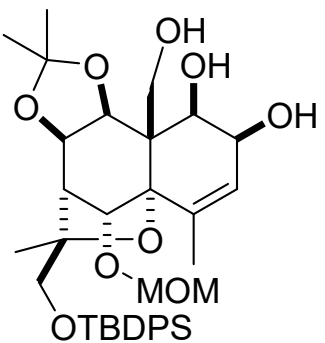

crude 39

${ }^{1} \mathrm{H}$ NMR (400 MHz, $\mathrm{CDCl}_{3}$ )

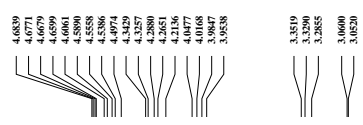

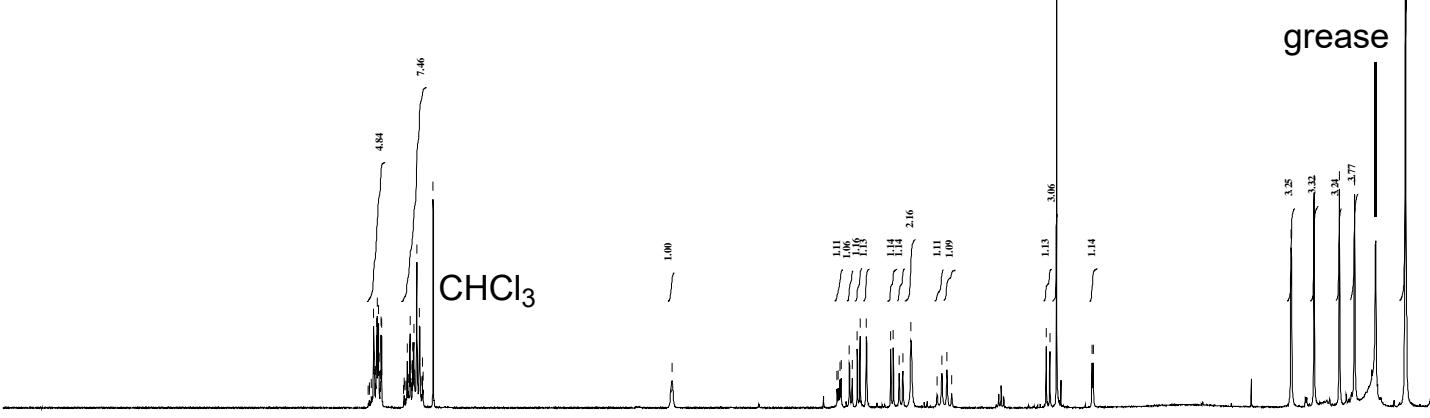

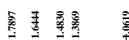

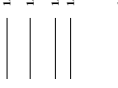

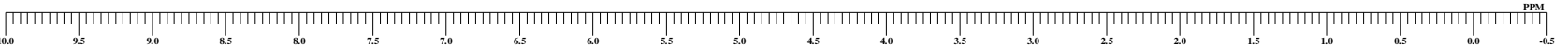

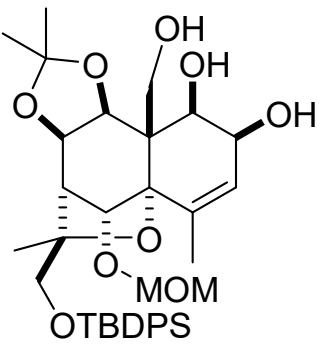

crude 39

${ }^{13} \mathrm{C}\left\{{ }^{1} \mathrm{H}\right\} \operatorname{NMR}\left(125 \mathrm{MHz}, \mathrm{CDCl}_{3}\right)$
$\mathrm{CDCl}_{3}$

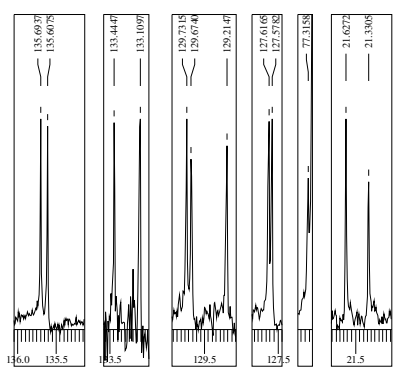




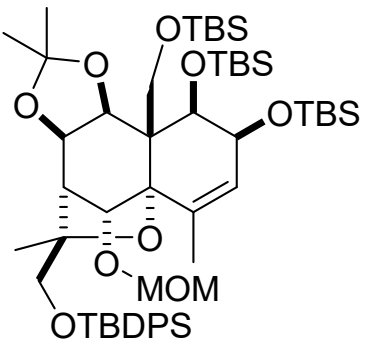

40

${ }^{1} \mathrm{H} \mathrm{NMR}\left(400 \mathrm{MHz}, \mathrm{CDCl}_{3}\right)$

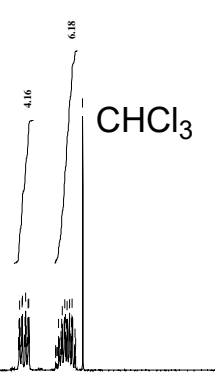

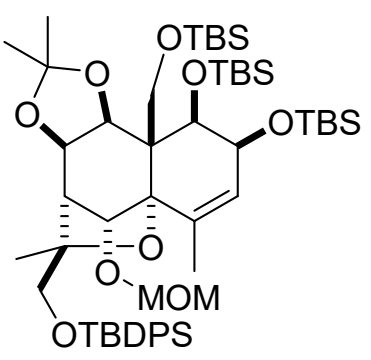

40

${ }^{13} \mathrm{C}\left\{{ }^{1} \mathrm{H}\right\}$ NMR $\left(125 \mathrm{MHz}, \mathrm{CDCl}_{3}\right)$

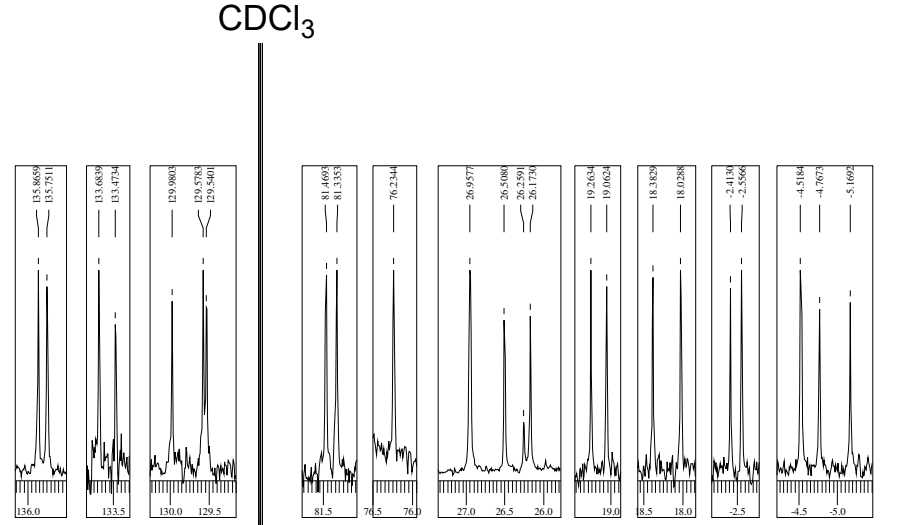

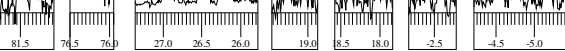

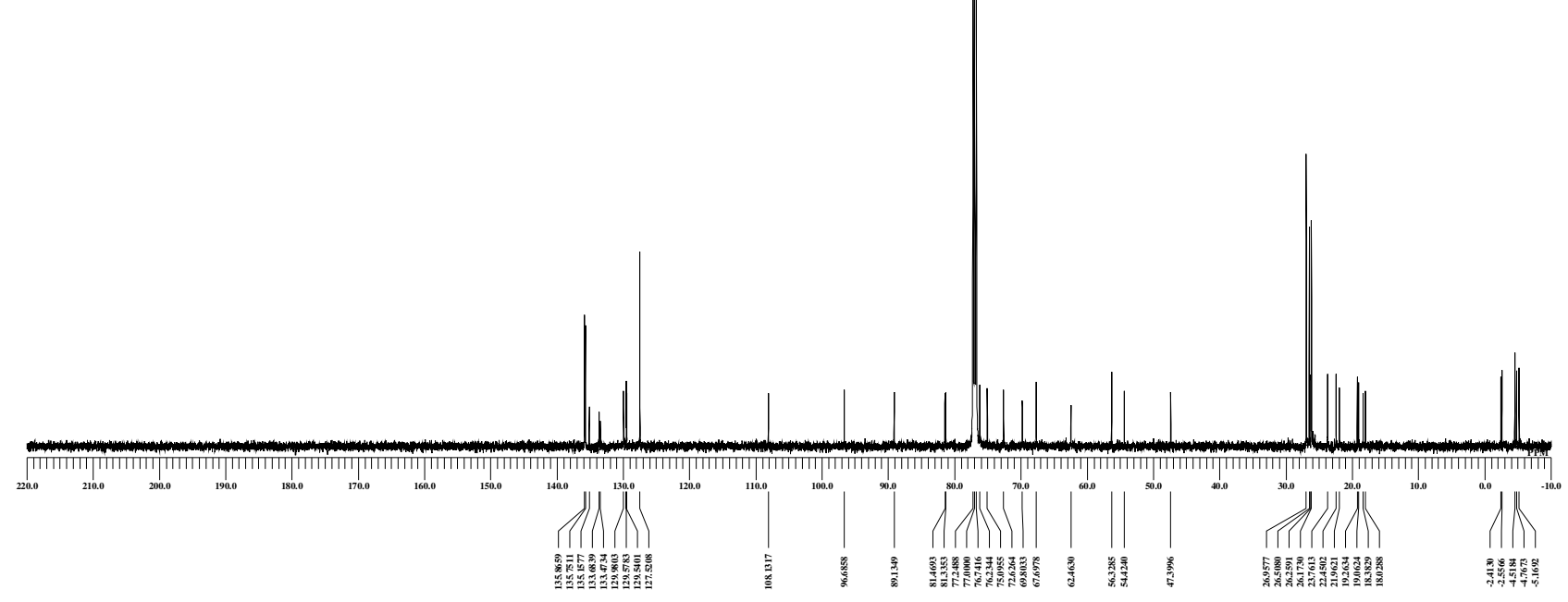




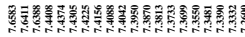

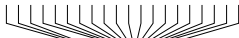

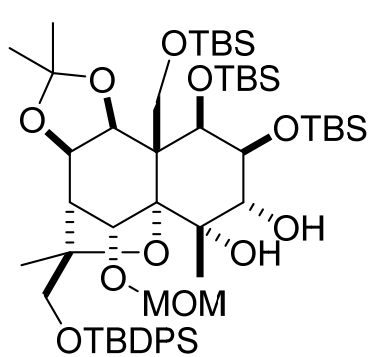

5

${ }^{1} \mathrm{H} \mathrm{NMR}\left(400 \mathrm{MHz}, \mathrm{CDCl}_{3}\right)$

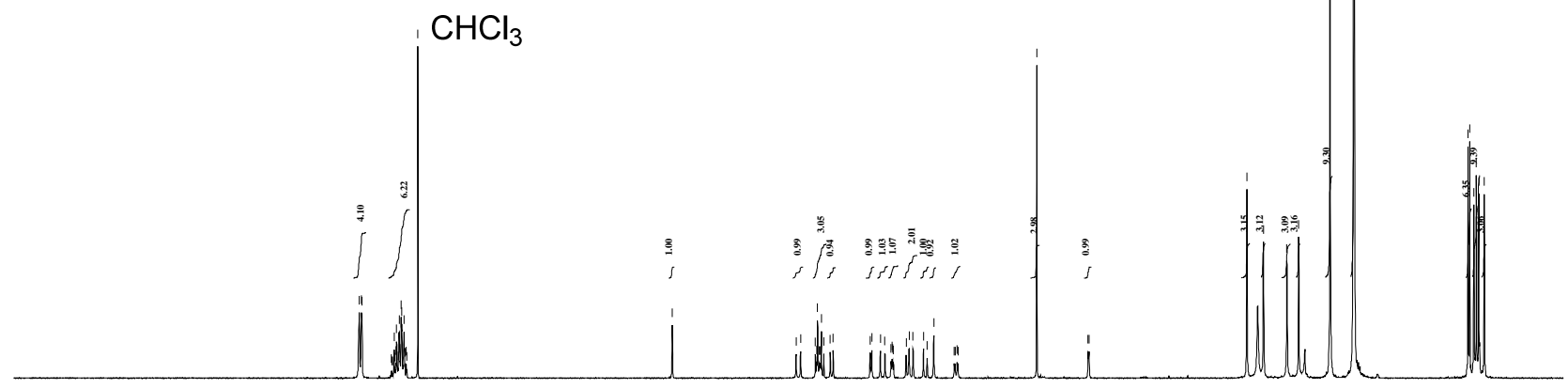

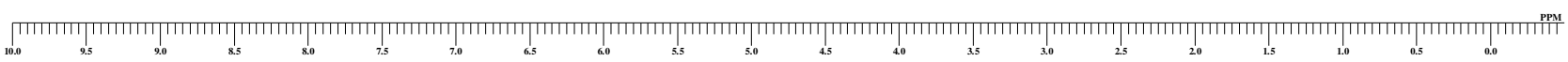

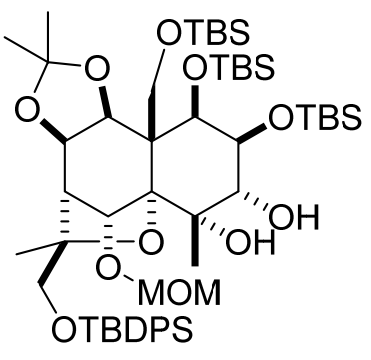

5

${ }^{13} \mathrm{C}\left\{{ }^{1} \mathrm{H}\right\} \operatorname{NMR}\left(125 \mathrm{MHz}, \mathrm{CDCl}_{3}\right)$
$\mathrm{CDCl}_{3}$

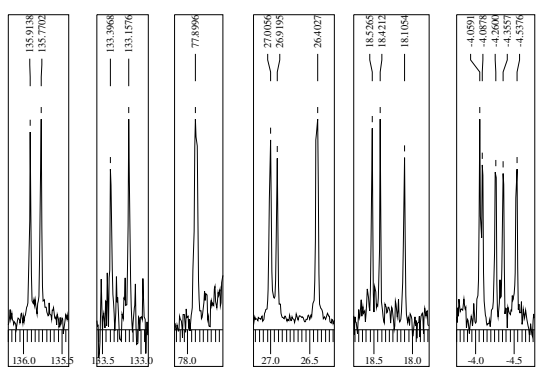

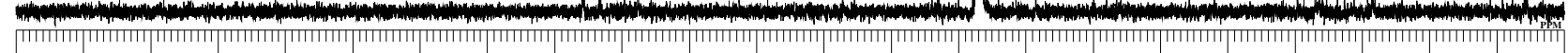

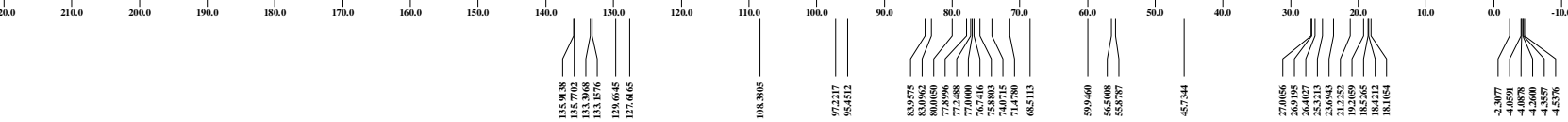


Supporting Information

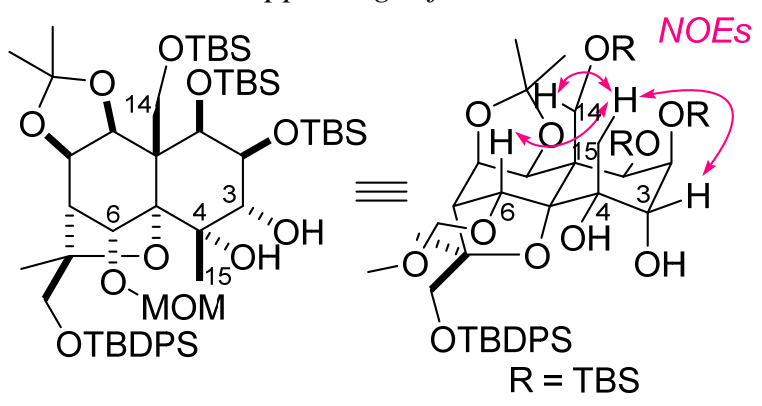

5

NOESY $\left(500 \mathrm{MHz}, \mathrm{CDCl}_{3}\right)$

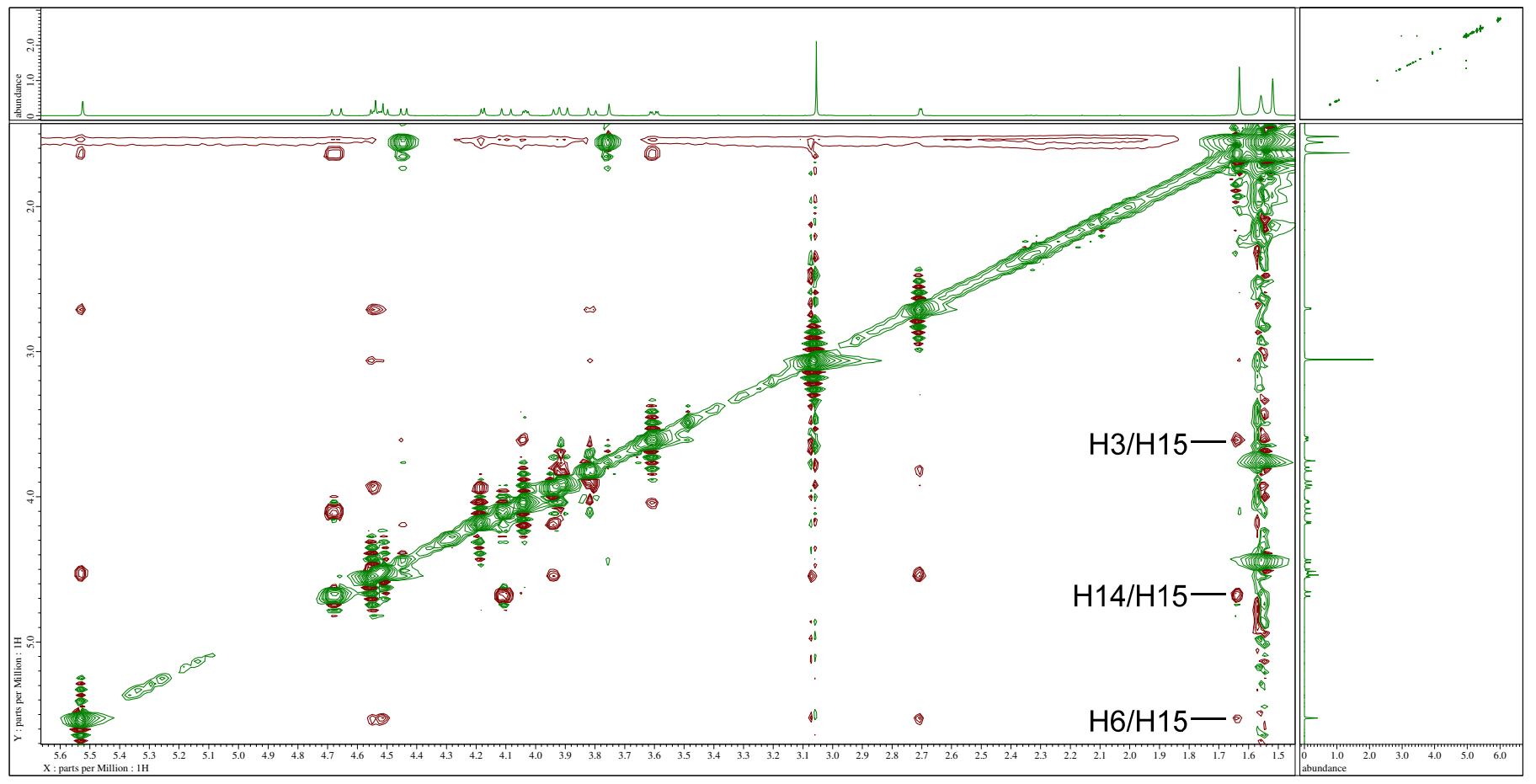




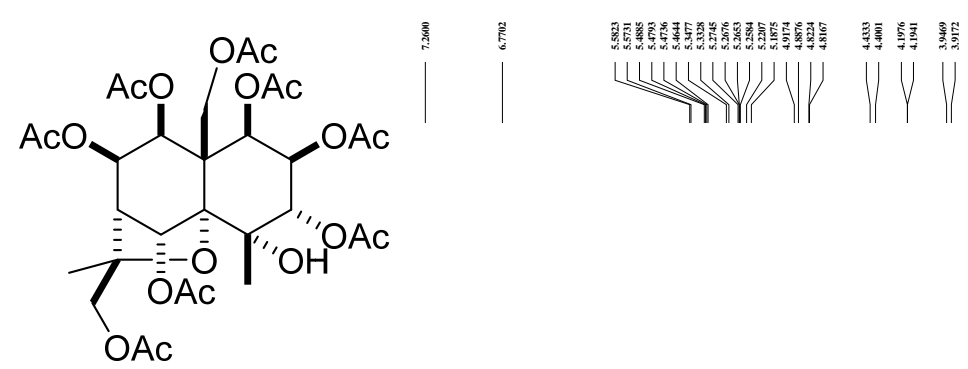

euonyminol octaacetate (2)

${ }^{1} \mathrm{H}$ NMR $\left(400 \mathrm{MHz}, \mathrm{CDCl}_{3}\right)$

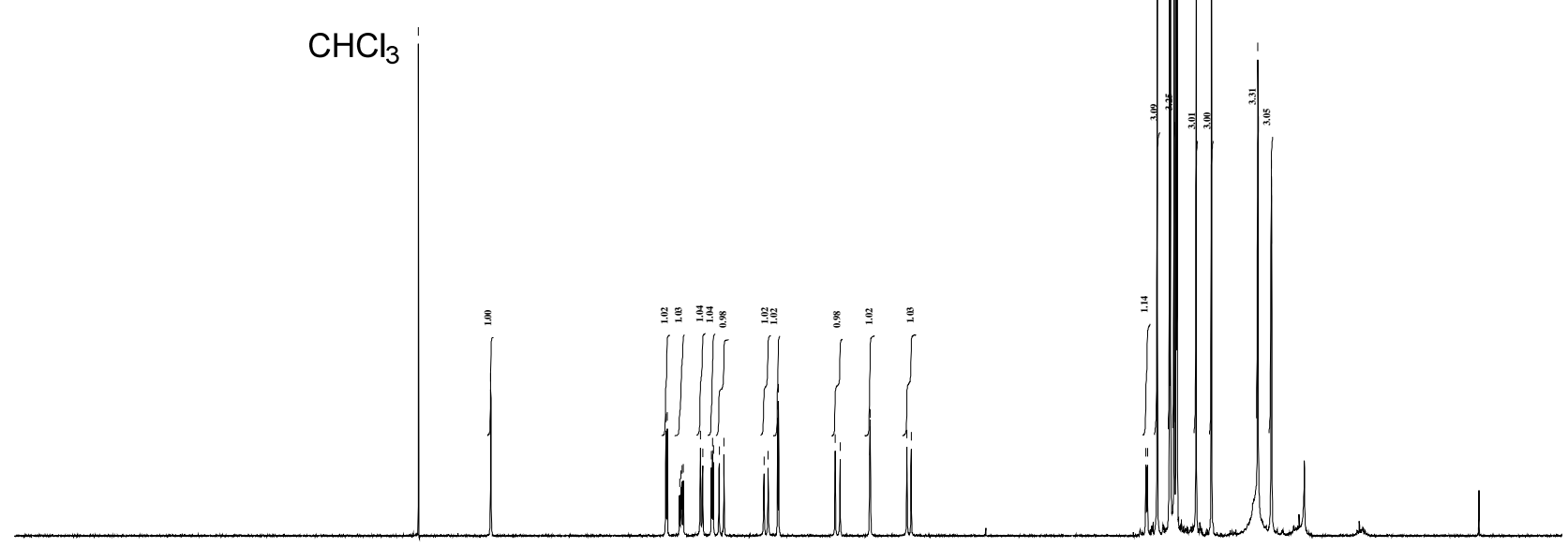

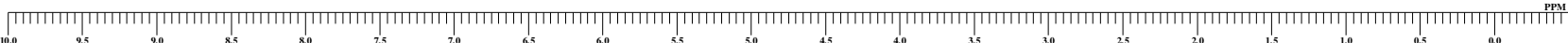

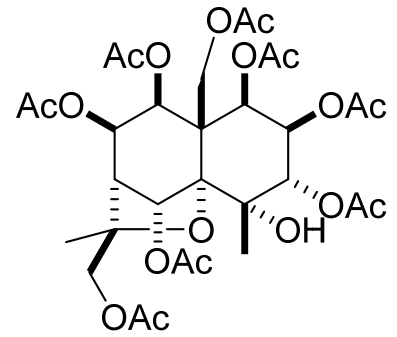

euonyminol octaacetate (2)

${ }^{13} \mathrm{C}\left\{{ }^{1} \mathrm{H}\right\}$ NMR $\left(125 \mathrm{MHz}, \mathrm{CDCl}_{3}\right)$
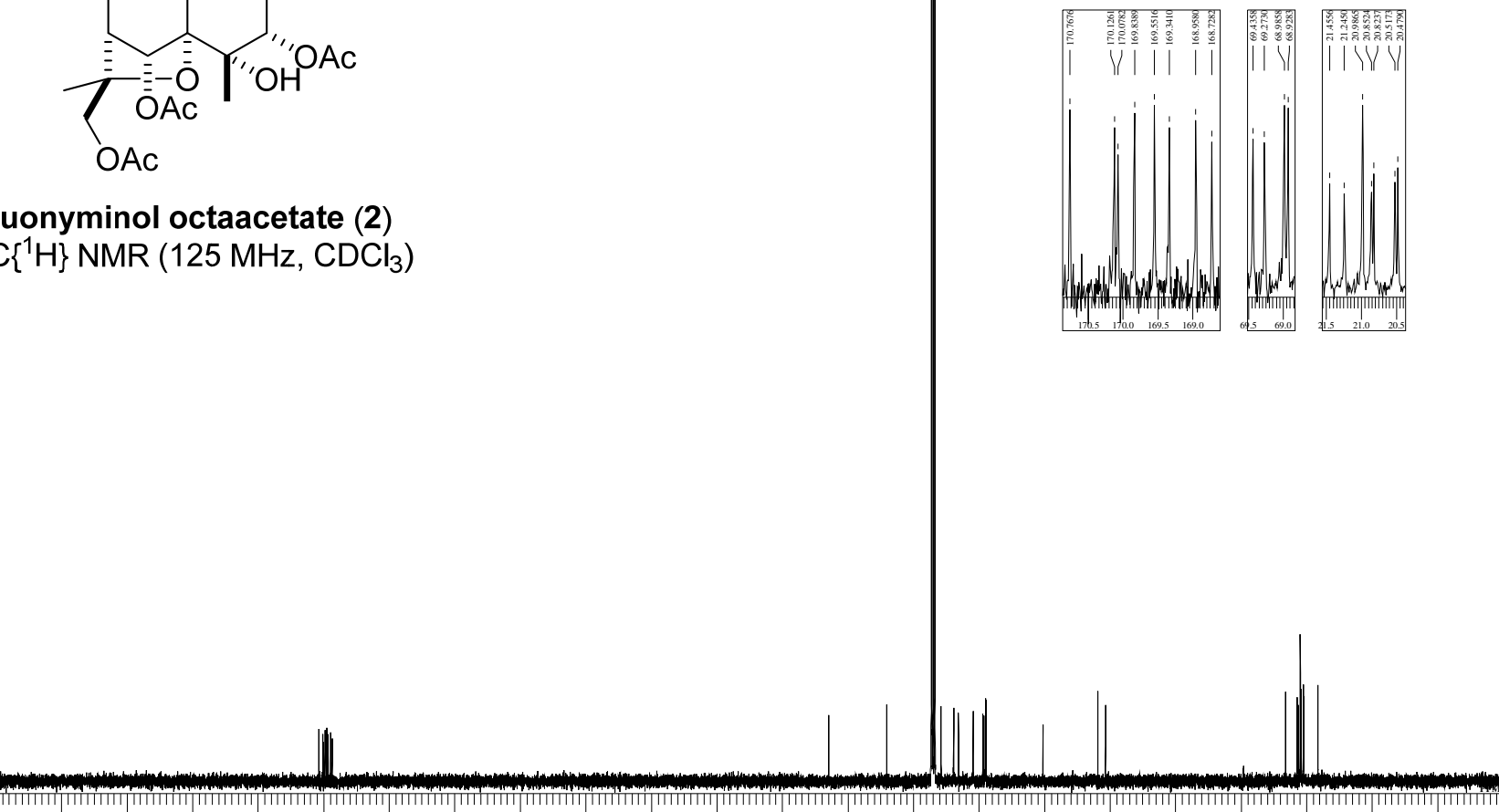

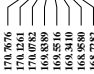

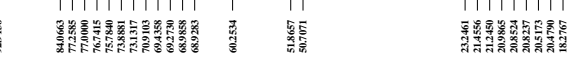




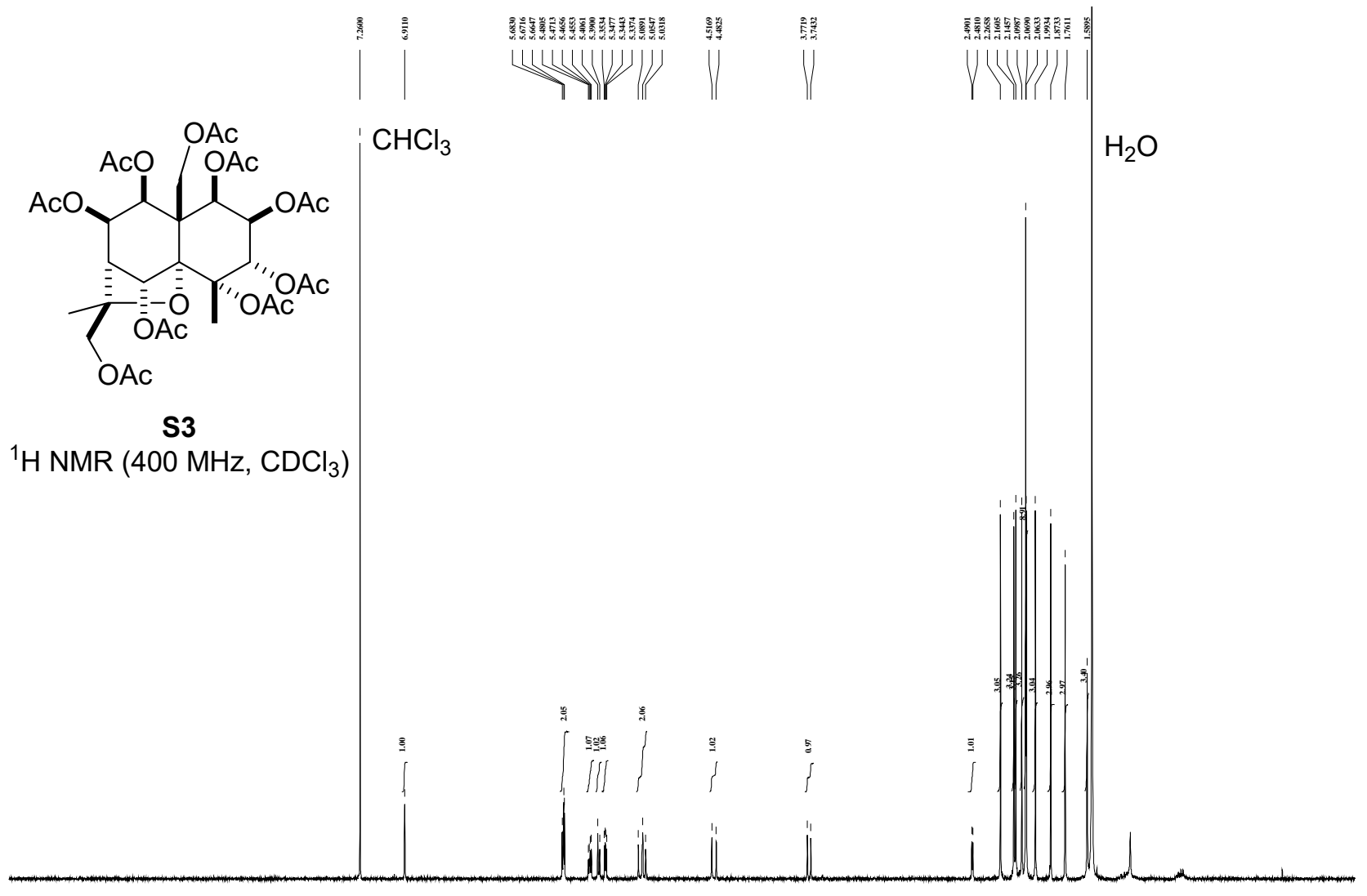

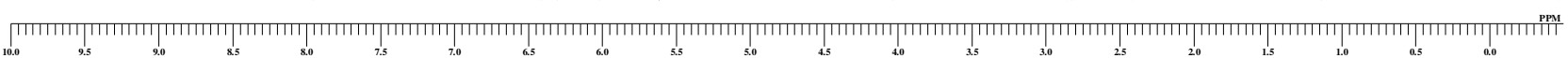

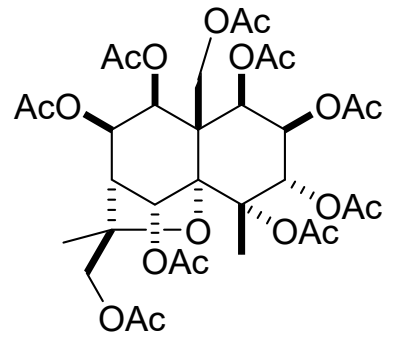

S3

${ }^{13} \mathrm{C}\left\{{ }^{1} \mathrm{H}\right\} \operatorname{NMR}\left(125 \mathrm{MHz}, \mathrm{CDCl}_{3}\right)$

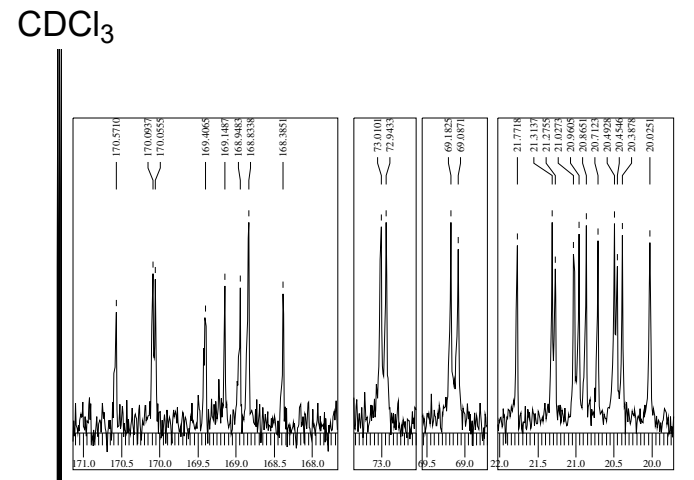

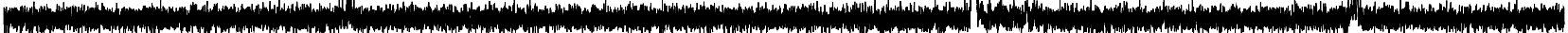

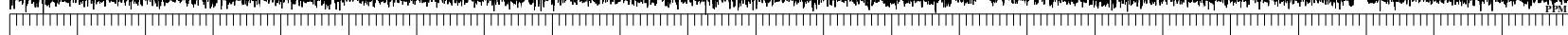
$220.0 \quad 210.0$ 

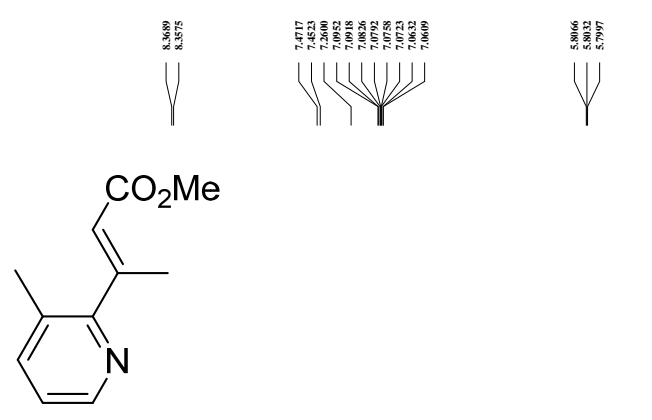

S5

${ }^{1} \mathrm{H}$ NMR $\left(400 \mathrm{MHz}, \mathrm{CDCl}_{3}\right)$

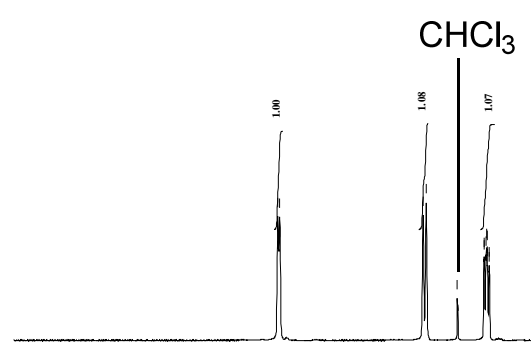

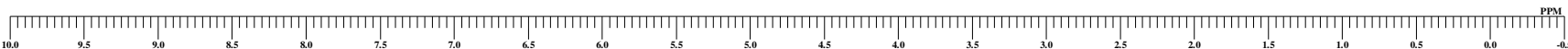<smiles>COC(=O)/C=C(\C)c1ncccc1C</smiles>

S5

${ }^{13} \mathrm{C}\left\{{ }^{1} \mathrm{H}\right\} \mathrm{NMR}\left(100 \mathrm{MHz}, \mathrm{CDCl}_{3}\right)$

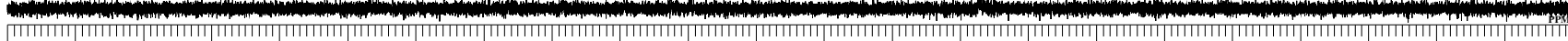
(100 


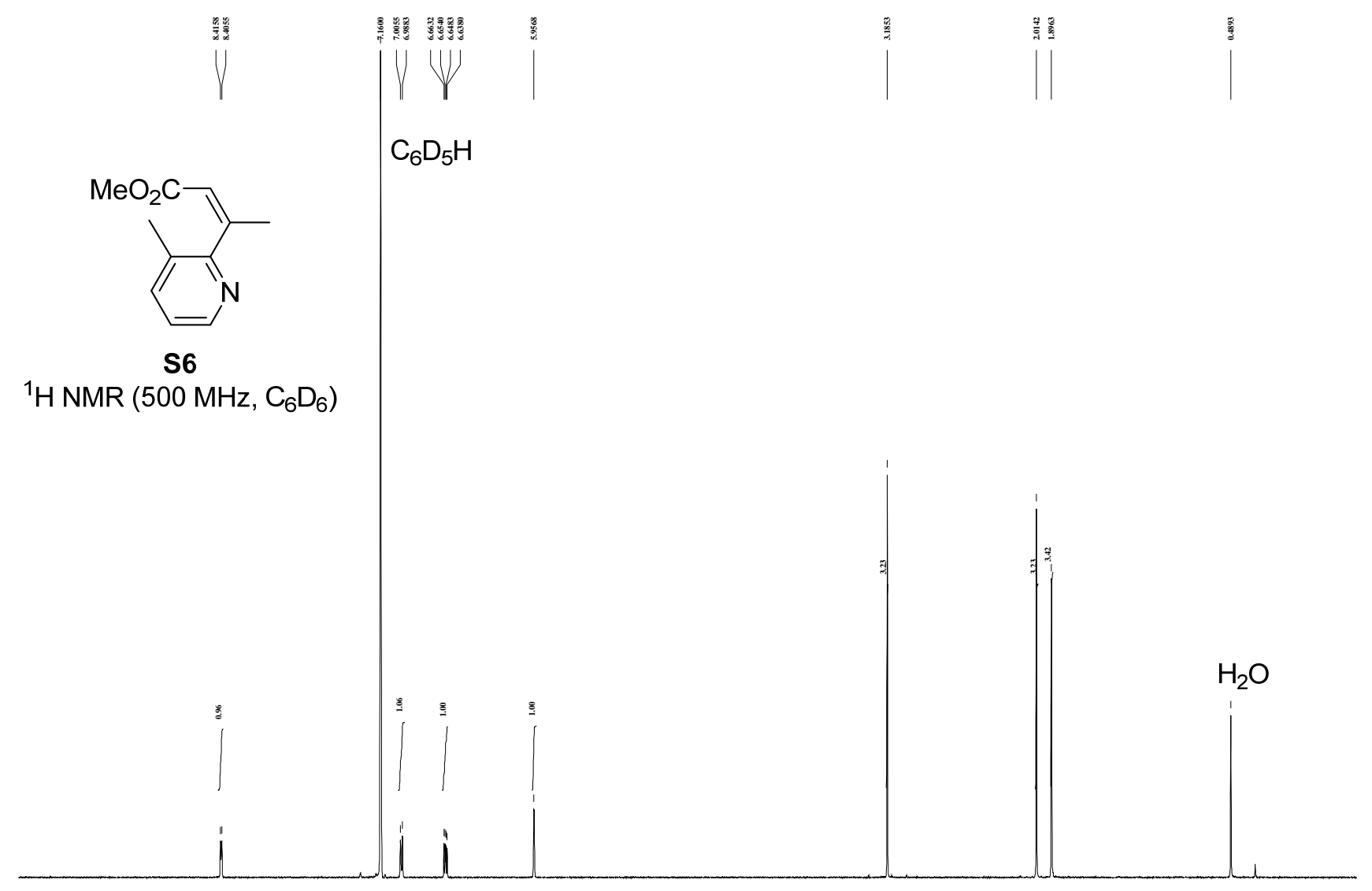

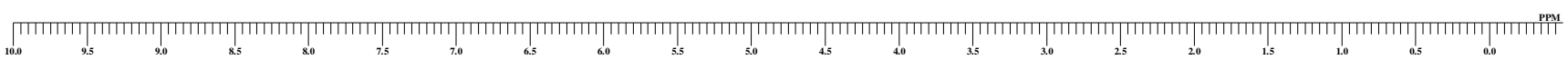

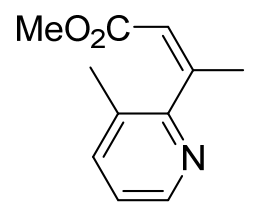

56

${ }^{13} \mathrm{C}\left\{{ }^{1} \mathrm{H}\right\} \mathrm{NMR}\left(100 \mathrm{MHz}, \mathrm{CDCl}_{3}\right)$

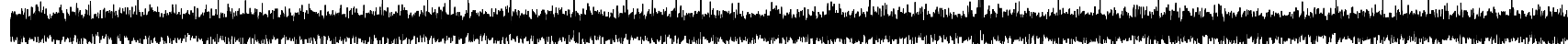

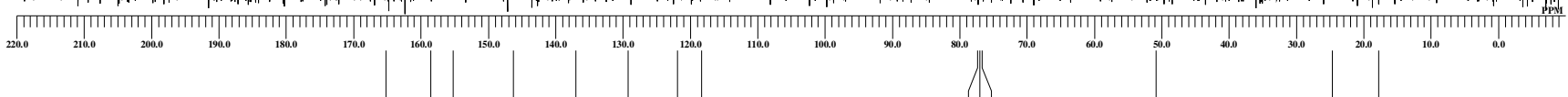

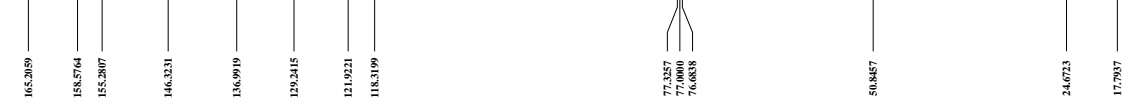




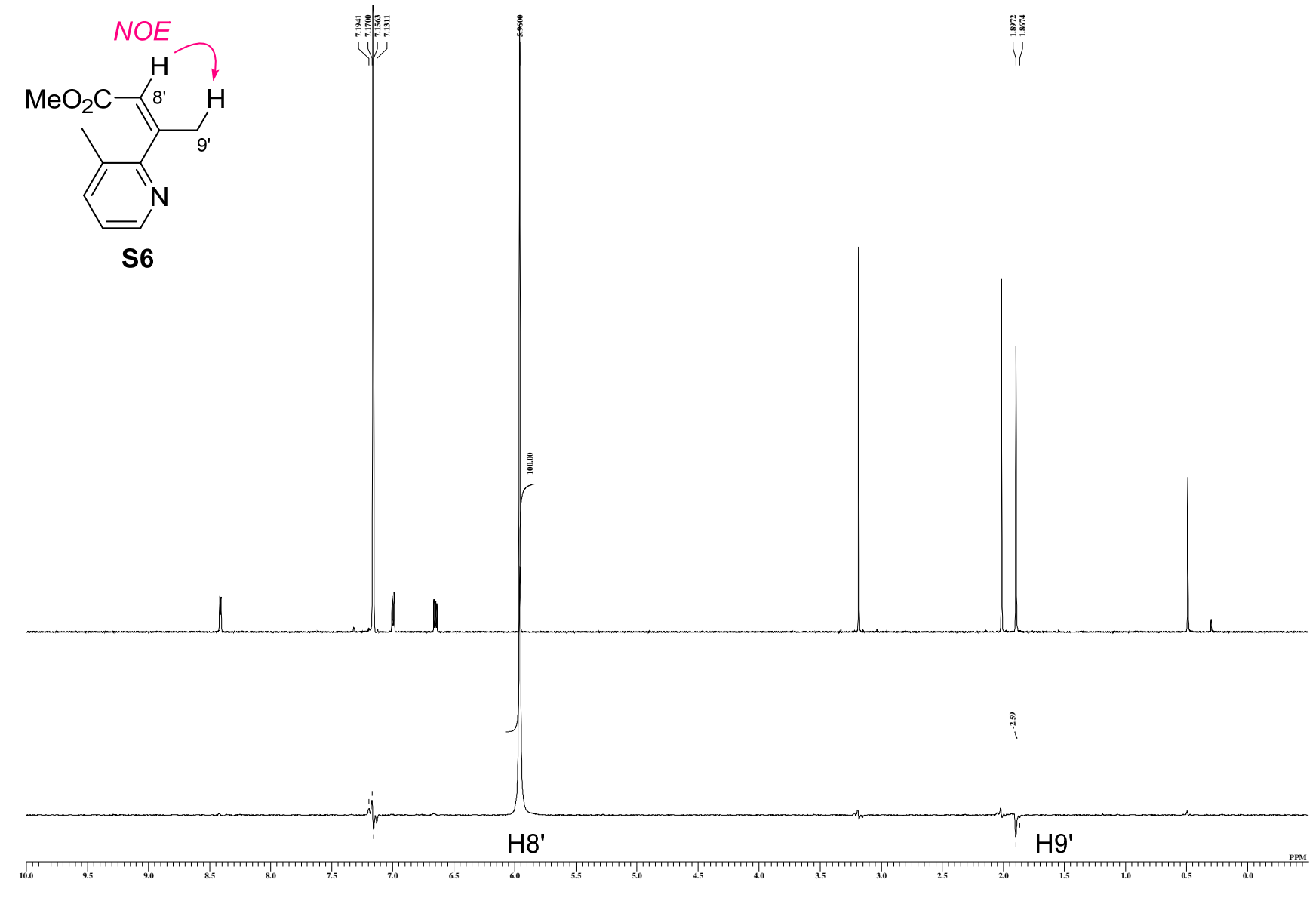




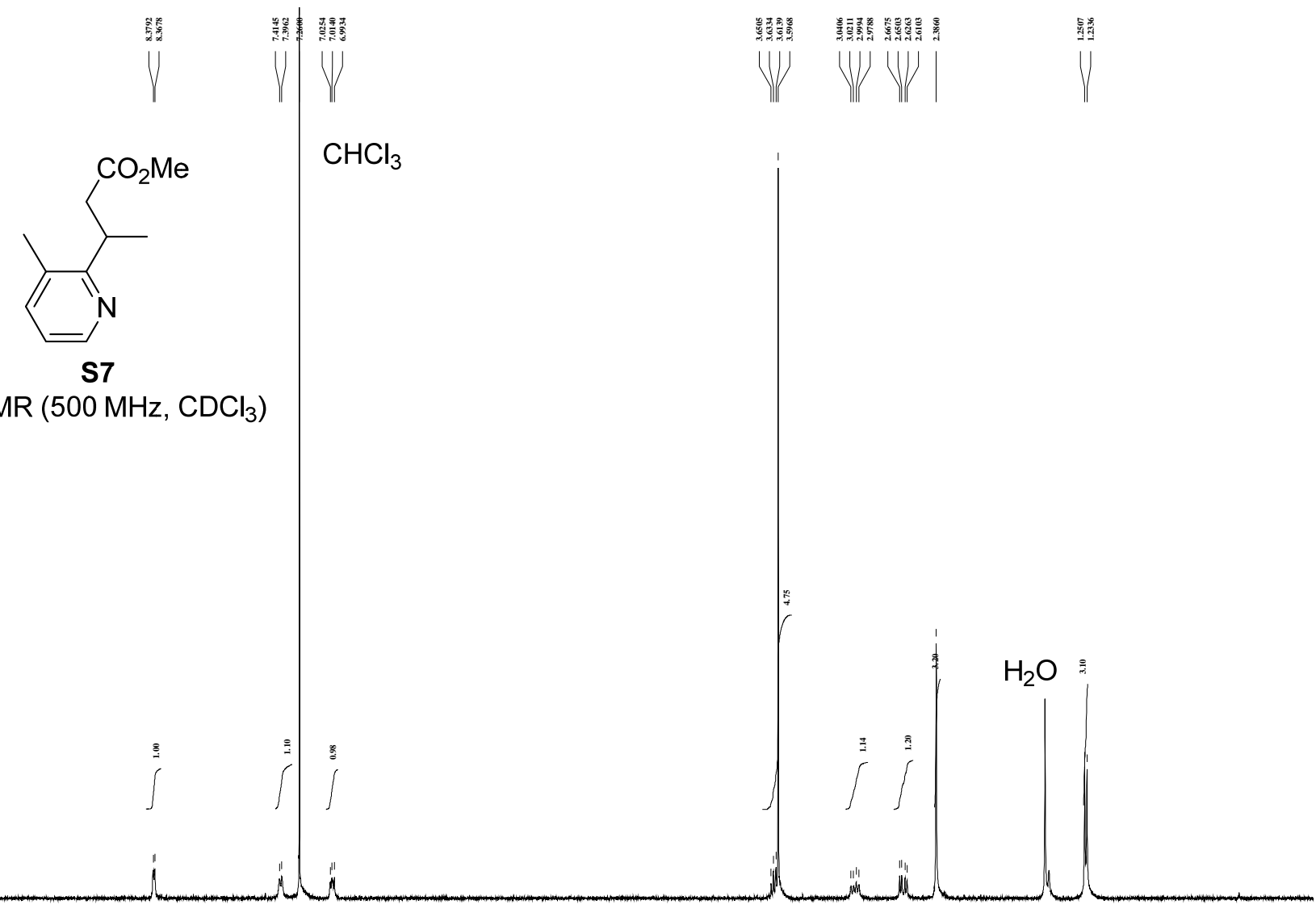

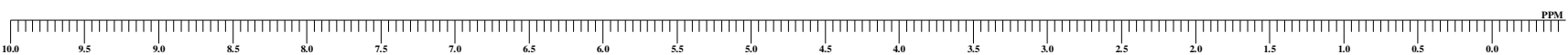

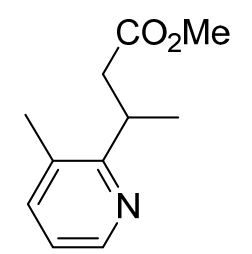

S7

${ }^{13} \mathrm{C}\left\{{ }^{1} \mathrm{H}\right\} \mathrm{NMR}\left(100 \mathrm{MHz}, \mathrm{CDCl}_{3}\right)$

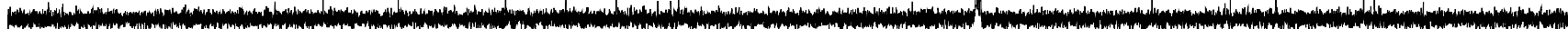

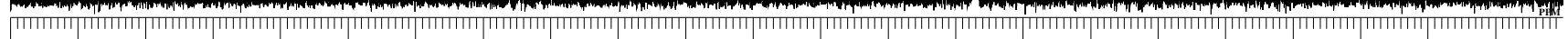

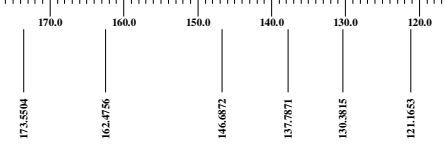




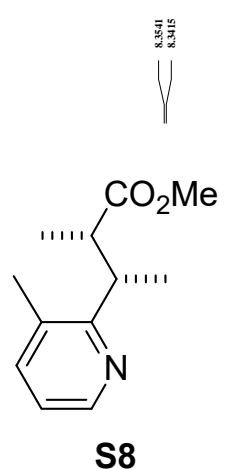

${ }^{1} \mathrm{H}$ NMR $\left(400 \mathrm{MHz}, \mathrm{CDCl}_{3}\right)$

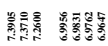

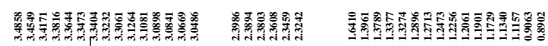

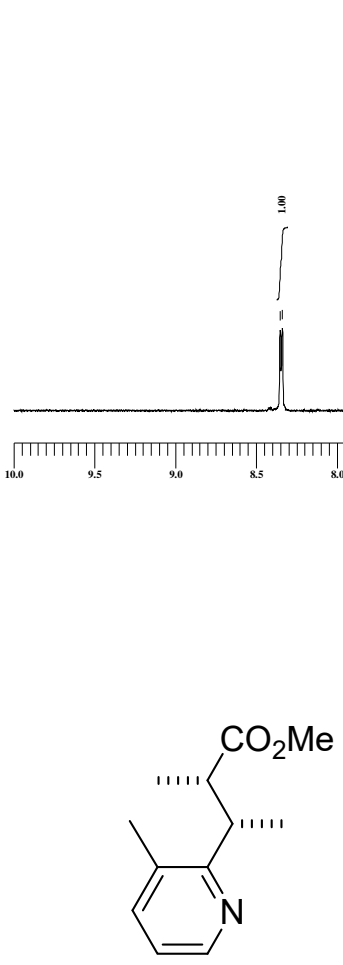

S8

${ }^{13} \mathrm{C}\left\{{ }^{1} \mathrm{H}\right\}$ NMR $\left(125 \mathrm{MHz}, \mathrm{CDCl}_{3}\right)$

$\mathrm{CDCl}_{3}$

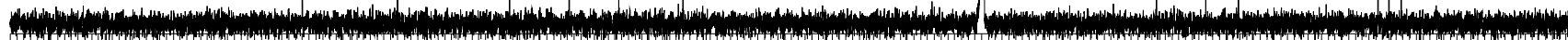

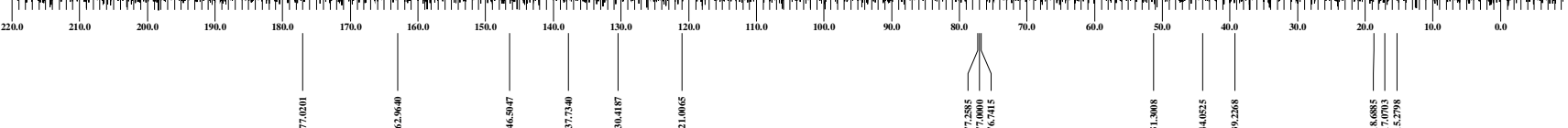




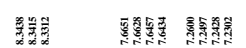

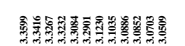

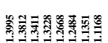

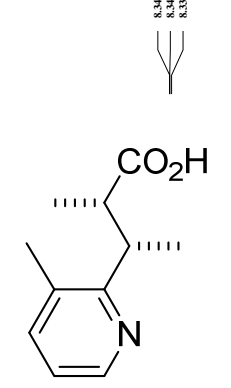

S9

${ }^{1} \mathrm{H}$ NMR (400 MHz, $\mathrm{CDCl}_{3}$ )

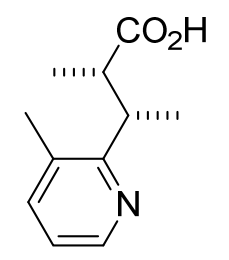

S9

$\mathrm{CHCl}_{3}$

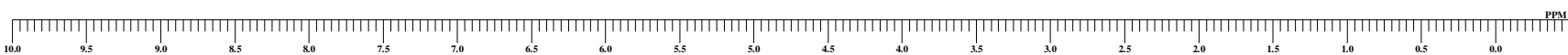
${ }^{13} \mathrm{C}\left\{{ }^{1} \mathrm{H}\right\} \mathrm{NMR}\left(125 \mathrm{MHz}, \mathrm{CDCl}_{3}\right)$

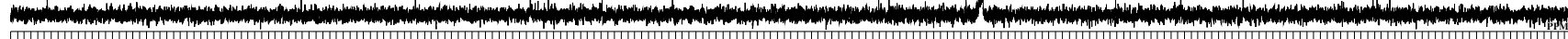

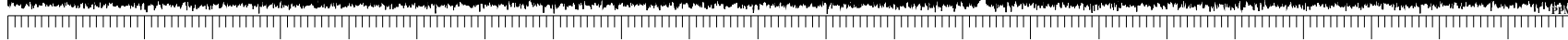
$\mathrm{CDCl}_{3}$ 


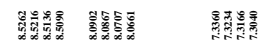<smiles>C[C@H](C(=O)O)[C@@H](C)c1ncccc1C(=O)O</smiles>

4

${ }^{1} \mathrm{H}$ NMR $\left(400 \mathrm{MHz}, \mathrm{CD}_{3} \mathrm{OD}\right)$

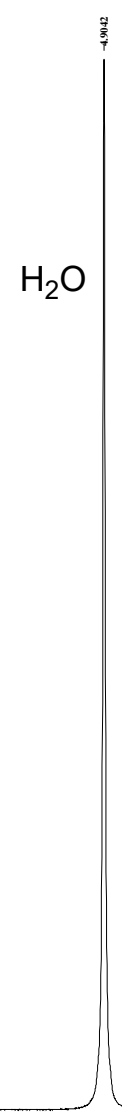

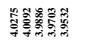

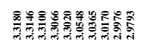

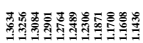
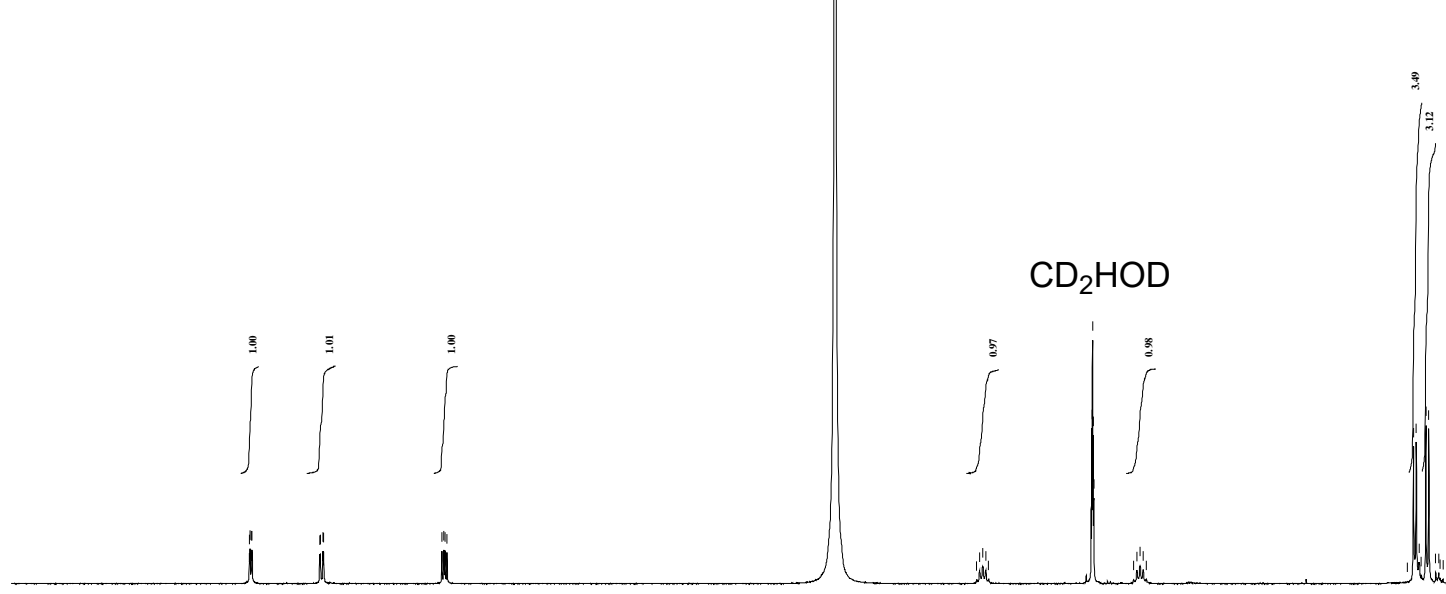

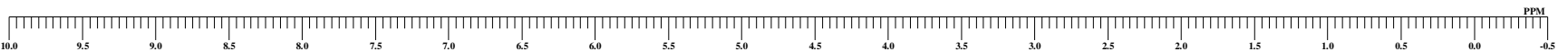
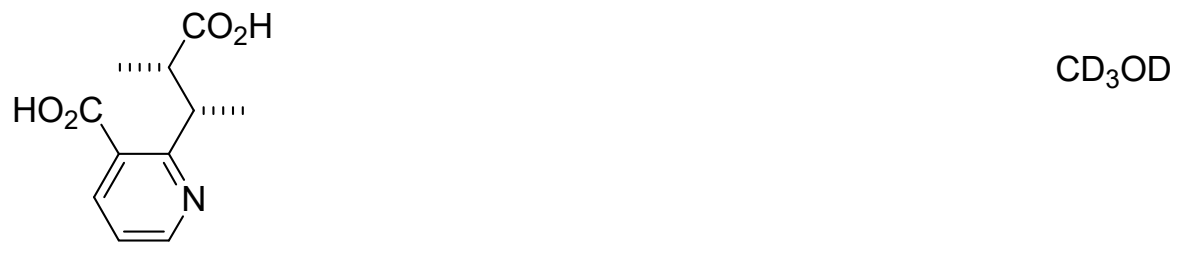

4

${ }^{13} \mathrm{C}\left\{{ }^{1} \mathrm{H}\right\}$ NMR $\left(125 \mathrm{MHz}, \mathrm{CD}_{3} \mathrm{OD}\right)$ 


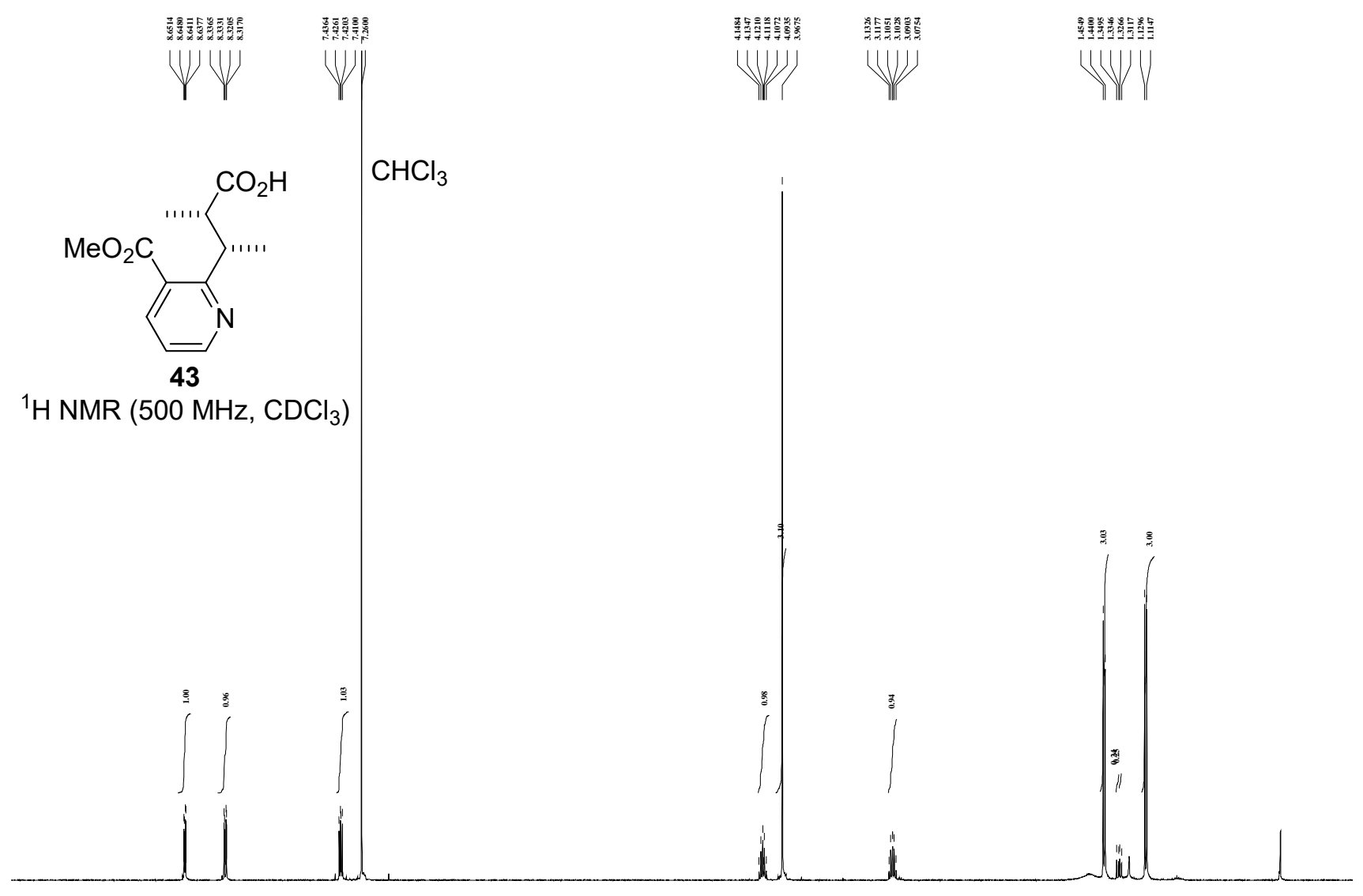

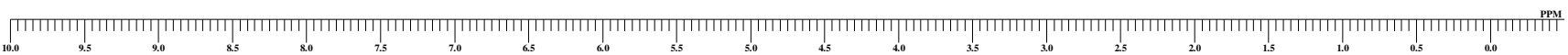<smiles>CC(=O)c1cccnc1[C@@H](C)[C@@H](C)C(=O)O</smiles>

43

${ }^{13} \mathrm{C}\left\{{ }^{1} \mathrm{H}\right\} \mathrm{NMR}\left(125 \mathrm{MHz}, \mathrm{CDCl}_{3}\right)$

$\mathrm{CDCl}_{3}$

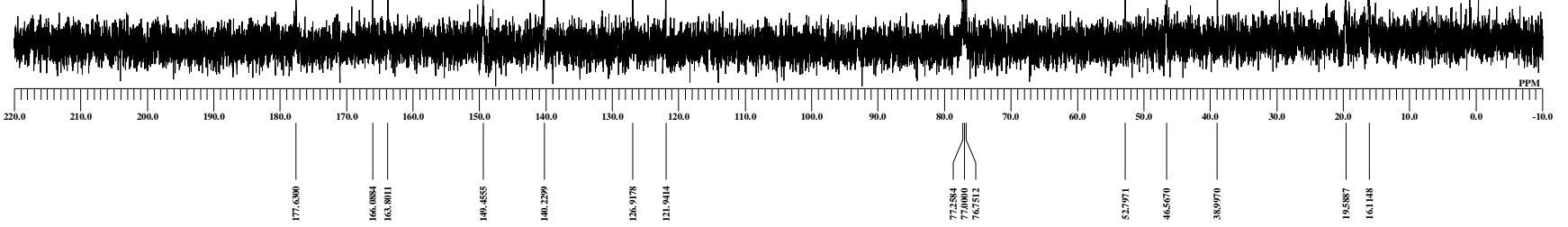



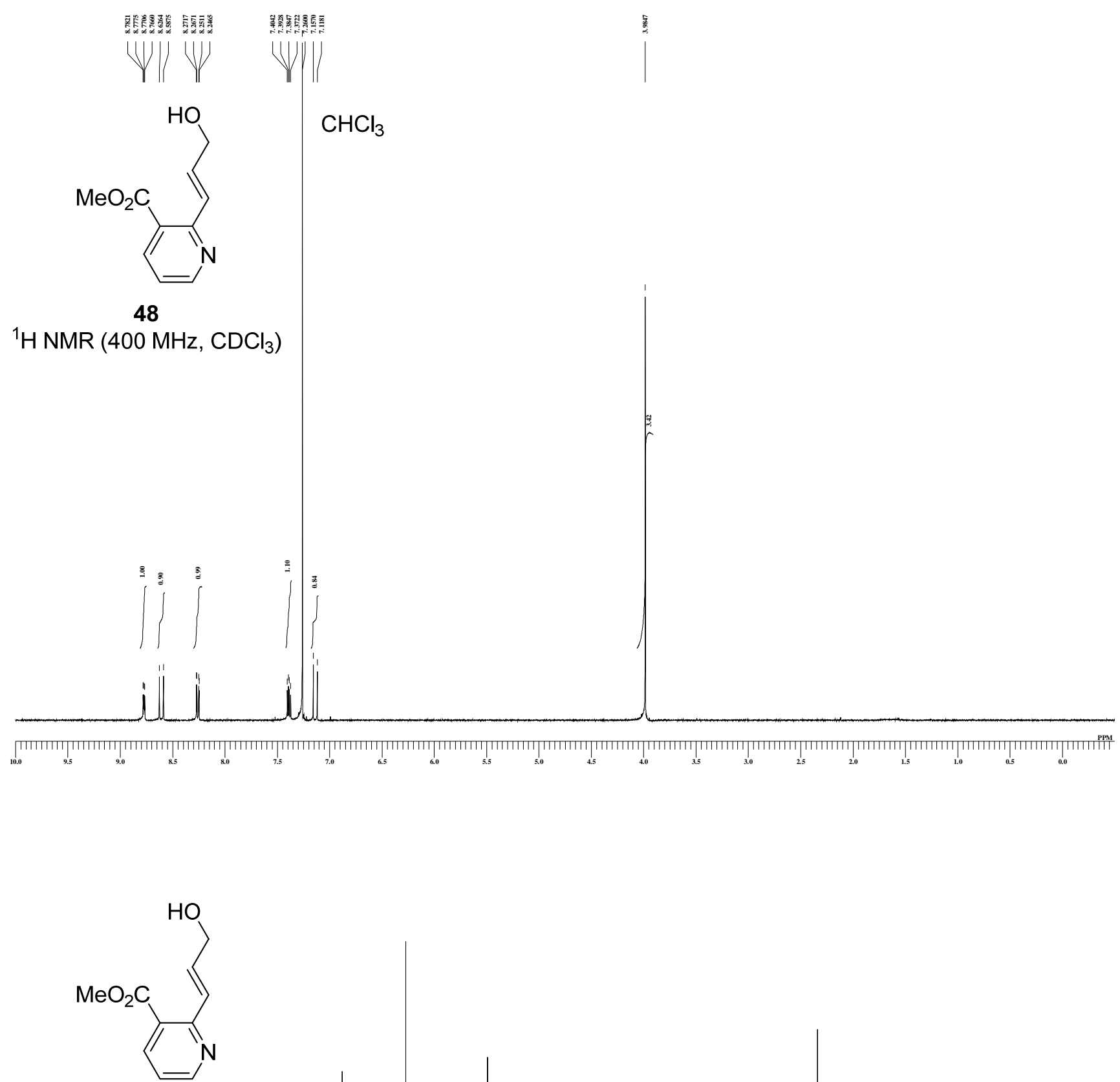

48

${ }^{13} \mathrm{C}\left\{{ }^{1} \mathrm{H}\right\} \operatorname{NMR}\left(100 \mathrm{MHz}, \mathrm{CDCl}_{3}\right)$

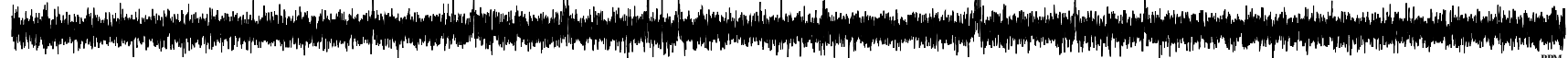
$\underset{22.0 .0}{\prod_{210.0}}$ 


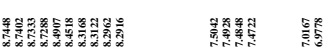<smiles>COC(=O)c1cccnc1/C=C/C(=O)O</smiles>

49

${ }^{1} \mathrm{H}$ NMR (400 MHz, $\left.\mathrm{CD}_{3} \mathrm{OD}\right)$

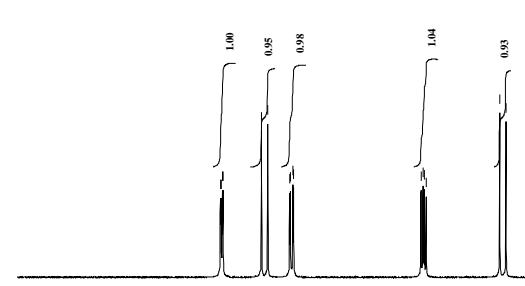

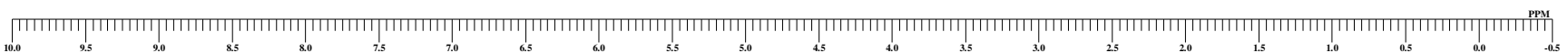

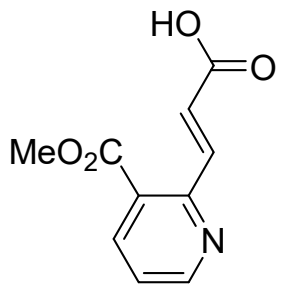

49

${ }^{13} \mathrm{C}\left\{{ }^{1} \mathrm{H}\right\} \mathrm{NMR}\left(100 \mathrm{MHz}, \mathrm{CD}_{3} \mathrm{OD}\right)$

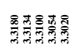

$\mathrm{CD}_{2} \mathrm{HOD}$

$\mathrm{H}_{2} \mathrm{O}$

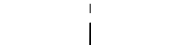


<smiles>CC(=O)c1cccnc1/C=C/C(=O)OC12CCC(CC1)C2(C)C</smiles>

50

${ }^{1} \mathrm{H}$ NMR $\left(500 \mathrm{MHz}, \mathrm{CDCl}_{3}\right)$

$\mathrm{CHCl}_{3}$

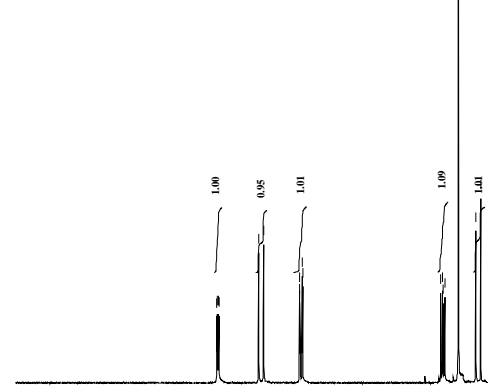

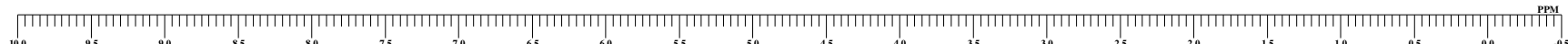

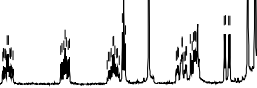

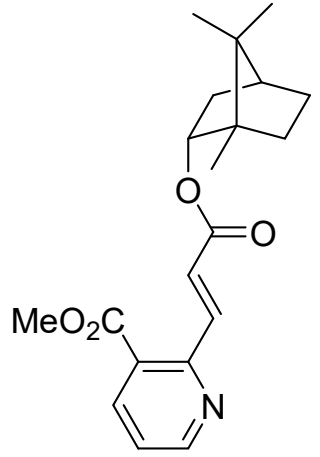

50

$\mathrm{CDCl}_{3}$

${ }^{13} \mathrm{C}\left\{{ }^{1} \mathrm{H}\right\} \operatorname{NMR}\left(125 \mathrm{MHz}, \mathrm{CDCl}_{3}\right)$
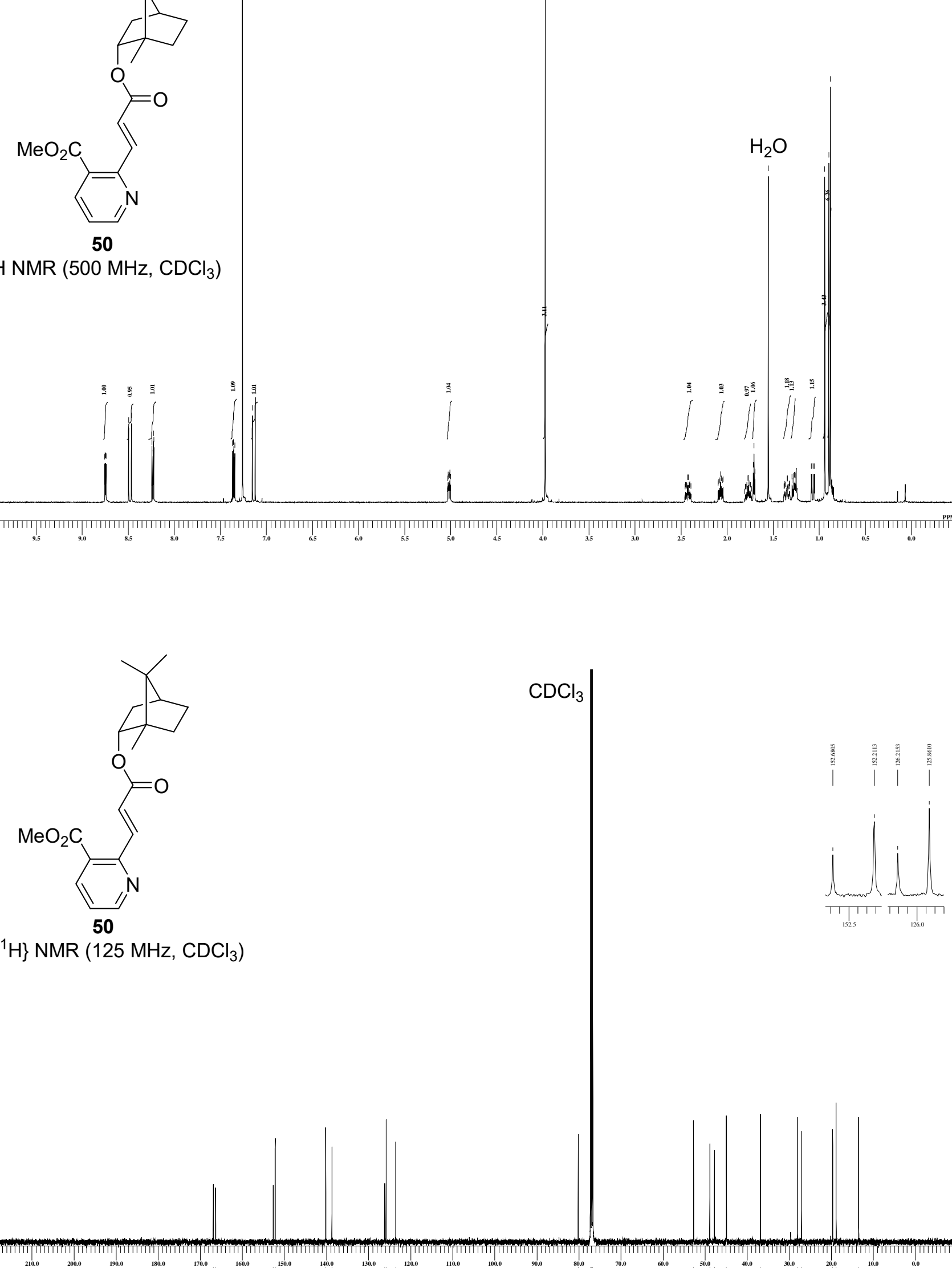

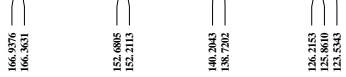

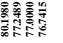

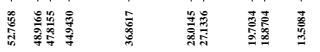




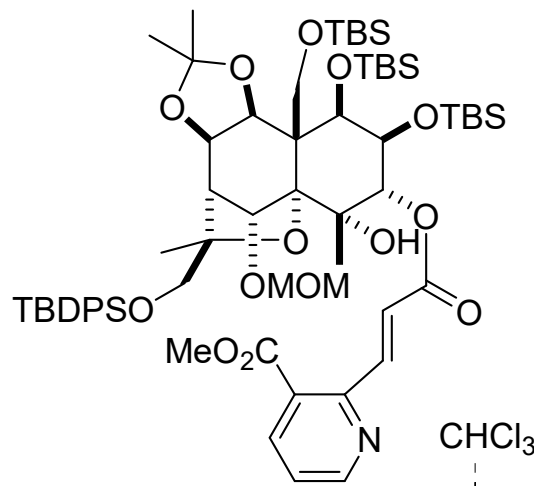

$\mathrm{H}_{2} \mathrm{O}$

\section{1}

${ }^{1} \mathrm{H}$ NMR $\left(400 \mathrm{MHz}, \mathrm{CDCl}_{3}\right)$

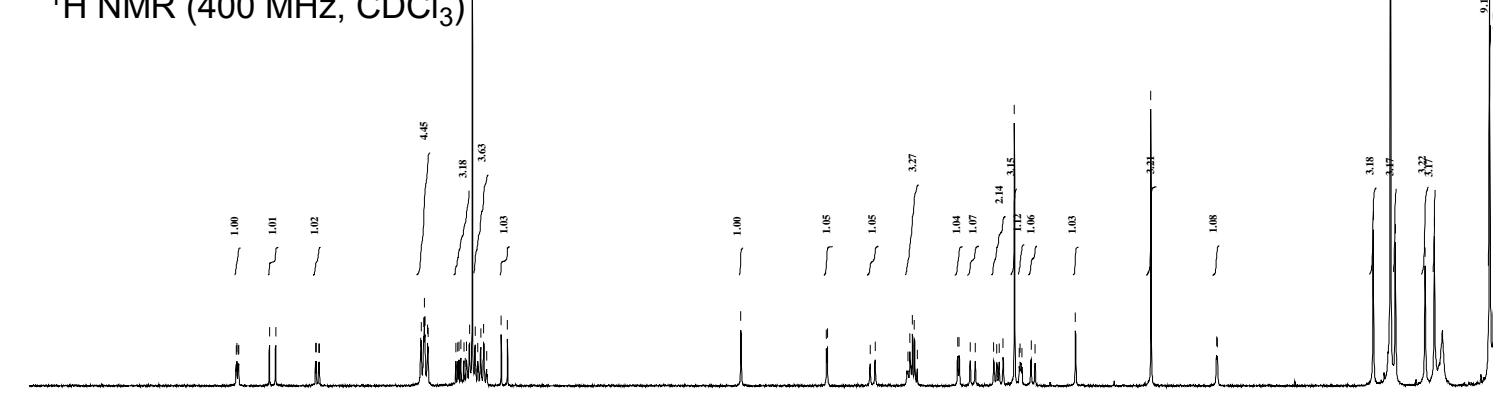

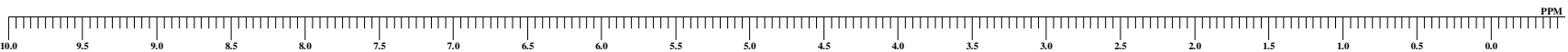

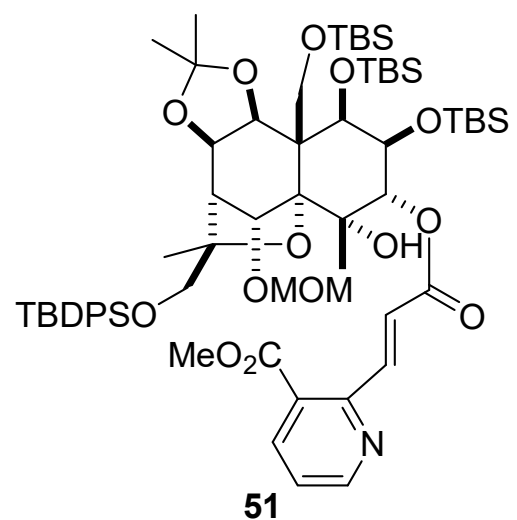

$\mathrm{CDCl}_{3}$

${ }^{13} \mathrm{C}\left\{{ }^{1} \mathrm{H}\right\}$ NMR $\left(100 \mathrm{MHz}, \mathrm{CDCl}_{3}\right)$
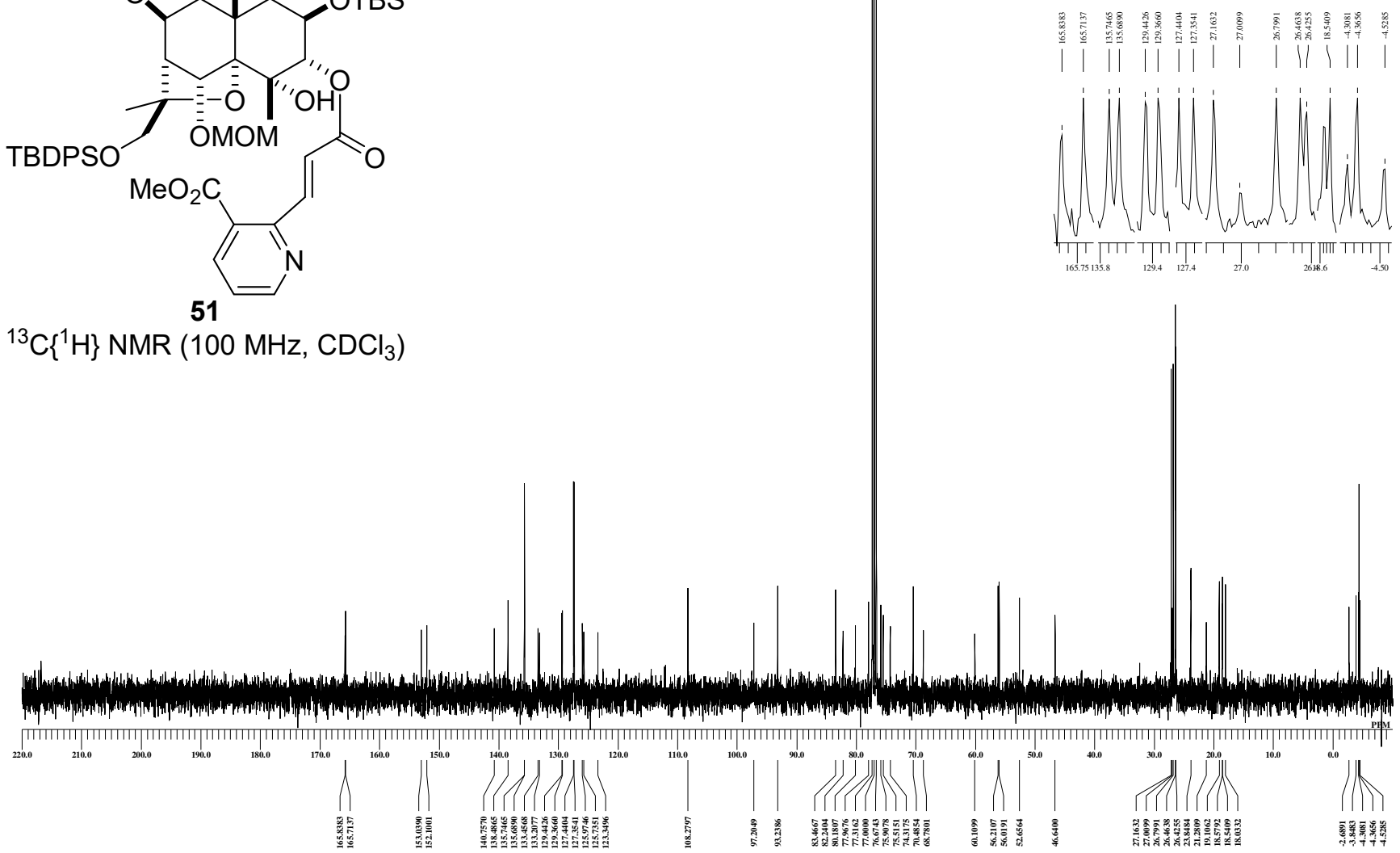


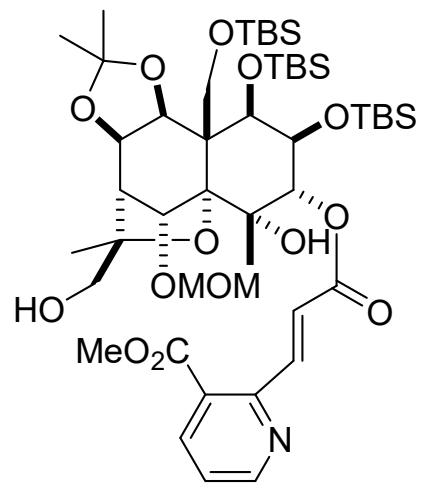

${ }^{1} \mathrm{H}$ NMR $\left(500 \mathrm{MHz}, \mathrm{CDCl}_{3}\right)$

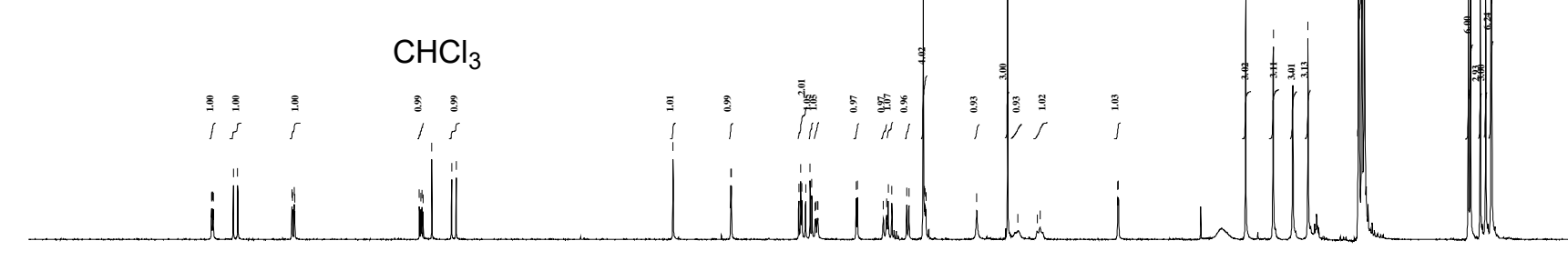

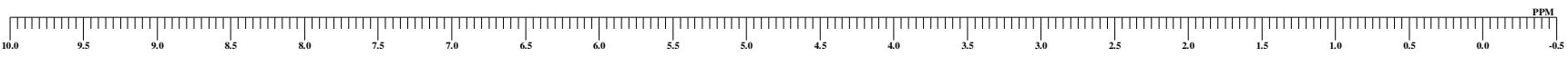
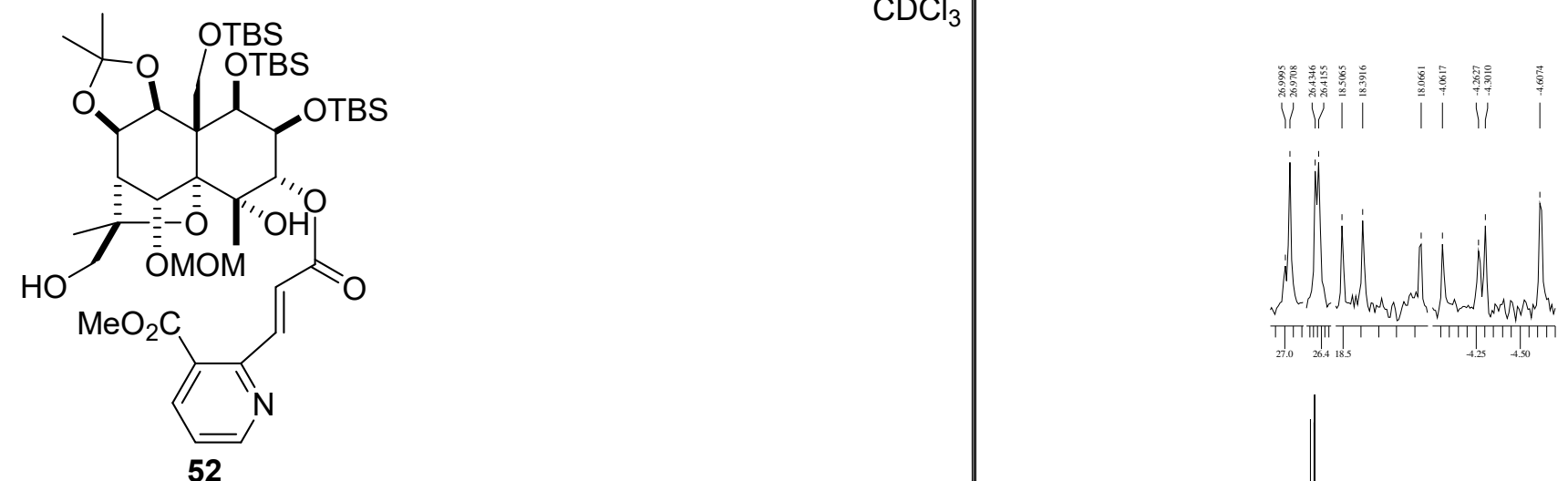

${ }^{13} \mathrm{C}\left\{{ }^{1} \mathrm{H}\right\}$ NMR $\left(125 \mathrm{MHz}, \mathrm{CDCl}_{3}\right)$ 


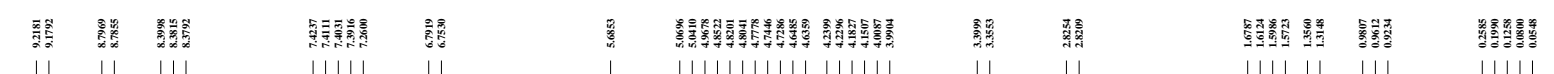

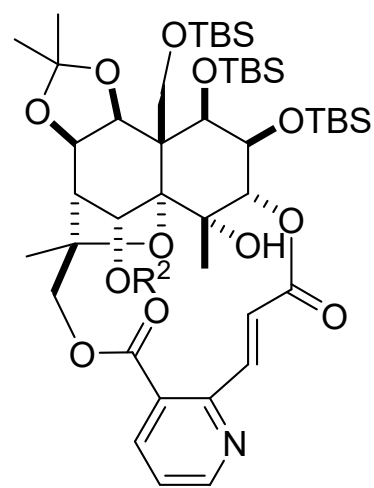

54: $\mathrm{R}^{2}=$ MOM

${ }^{1} \mathrm{H}$ NMR $\left(400 \mathrm{MHz}, \mathrm{CDCl}_{3}\right)$

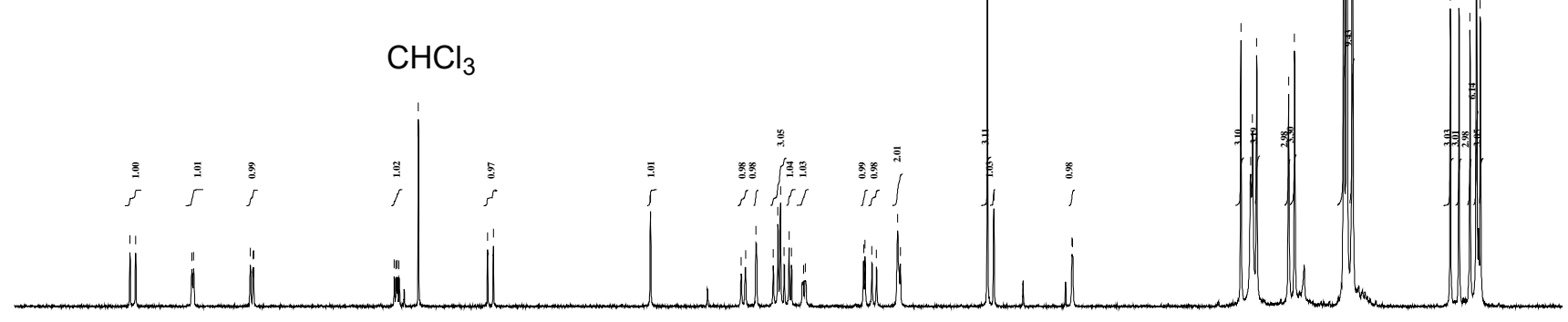

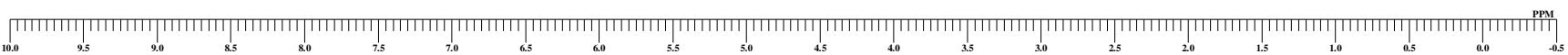
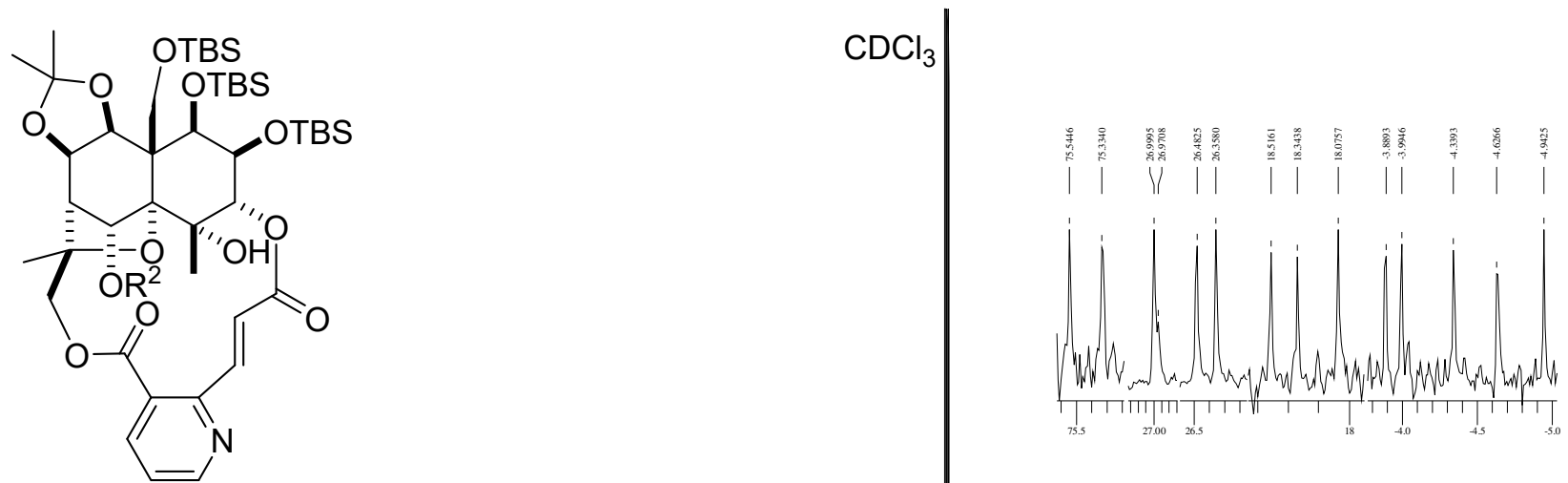

54: $R^{2}=$ MOM

${ }^{13} \mathrm{C}\left\{{ }^{1} \mathrm{H}\right\}$ NMR $\left(125 \mathrm{MHz}, \mathrm{CDCl}_{3}\right)$ 

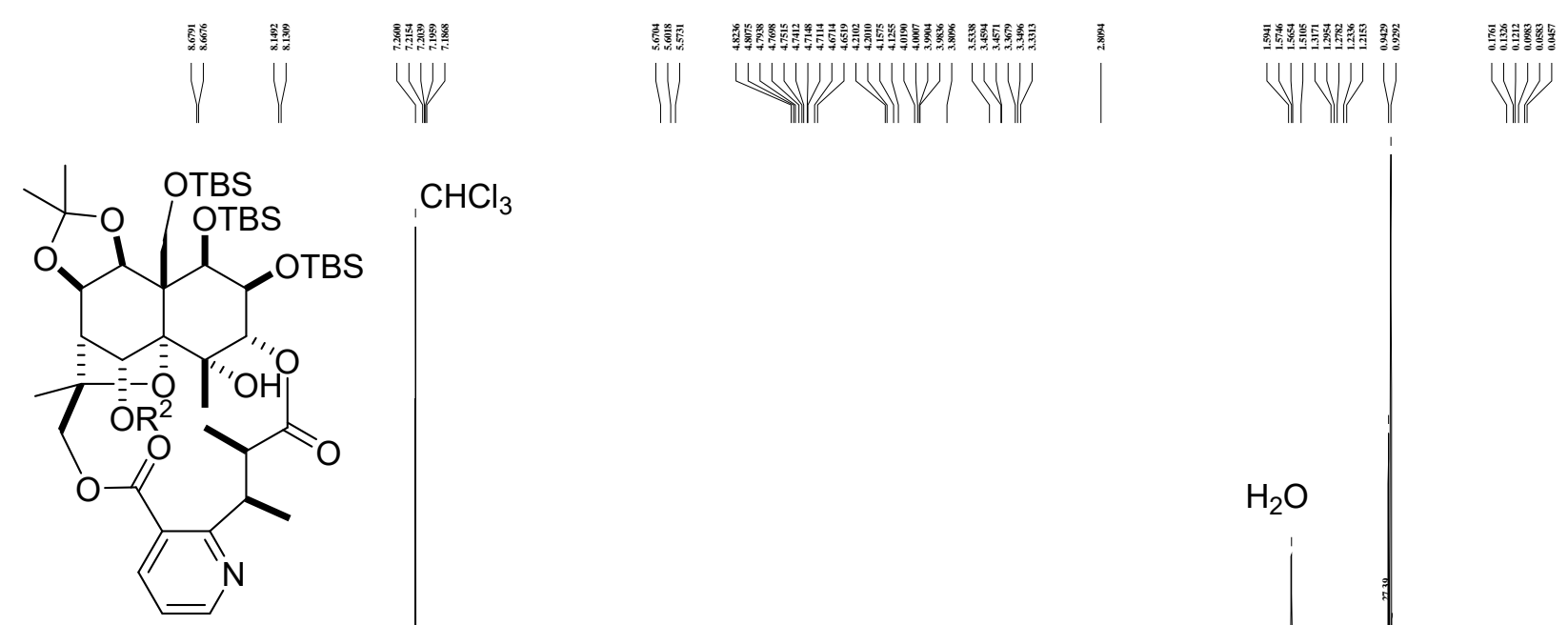

$\mathrm{CHCl}_{3}$

56: $\mathrm{R}^{2}=\mathrm{MOM}$

${ }^{1} \mathrm{H}$ NMR $\left(400 \mathrm{MHz}, \mathrm{CDCl}_{3}\right)$

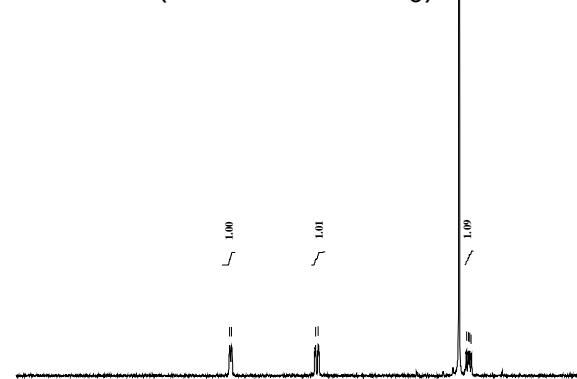

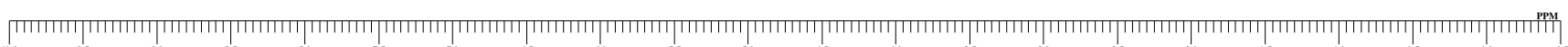
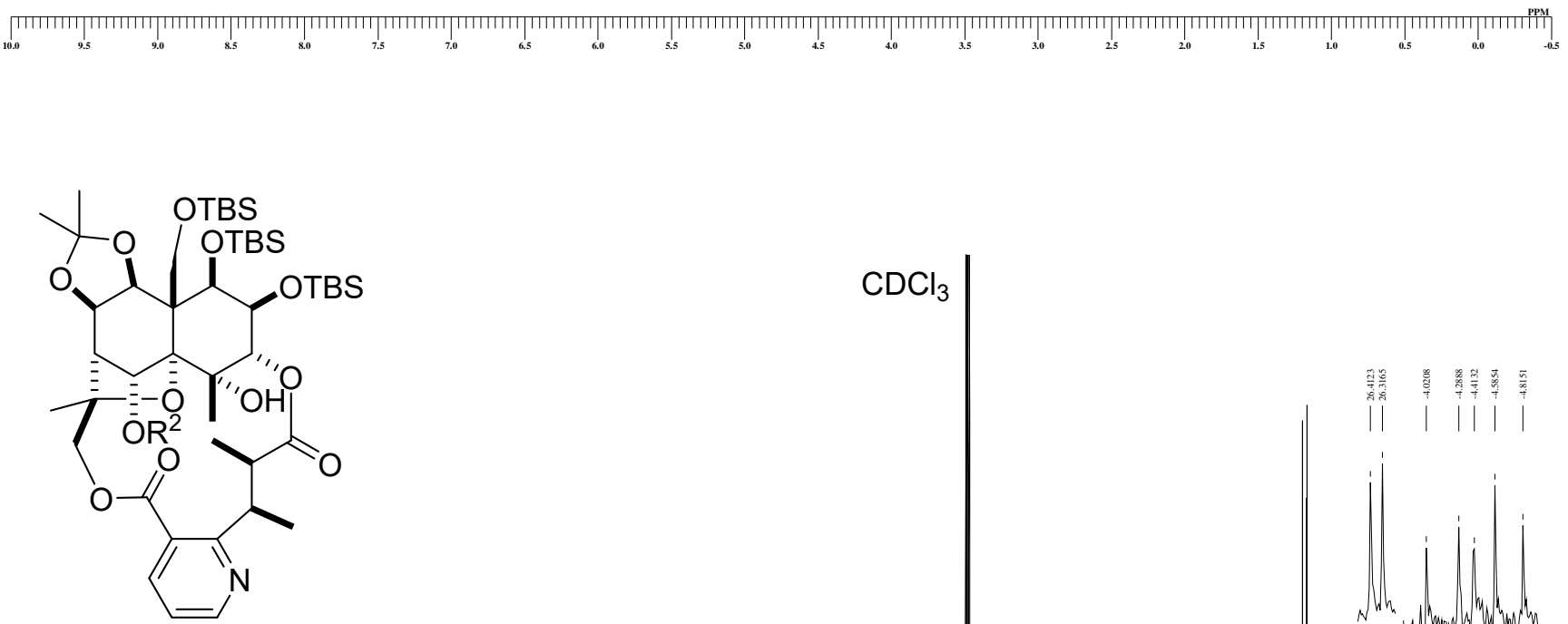

56: $\mathrm{R}^{2}=\mathrm{MOM}$

${ }^{13} \mathrm{C}\left\{{ }^{1} \mathrm{H}\right\} \operatorname{NMR}\left(125 \mathrm{MHz}, \mathrm{CDCl}_{3}\right)$

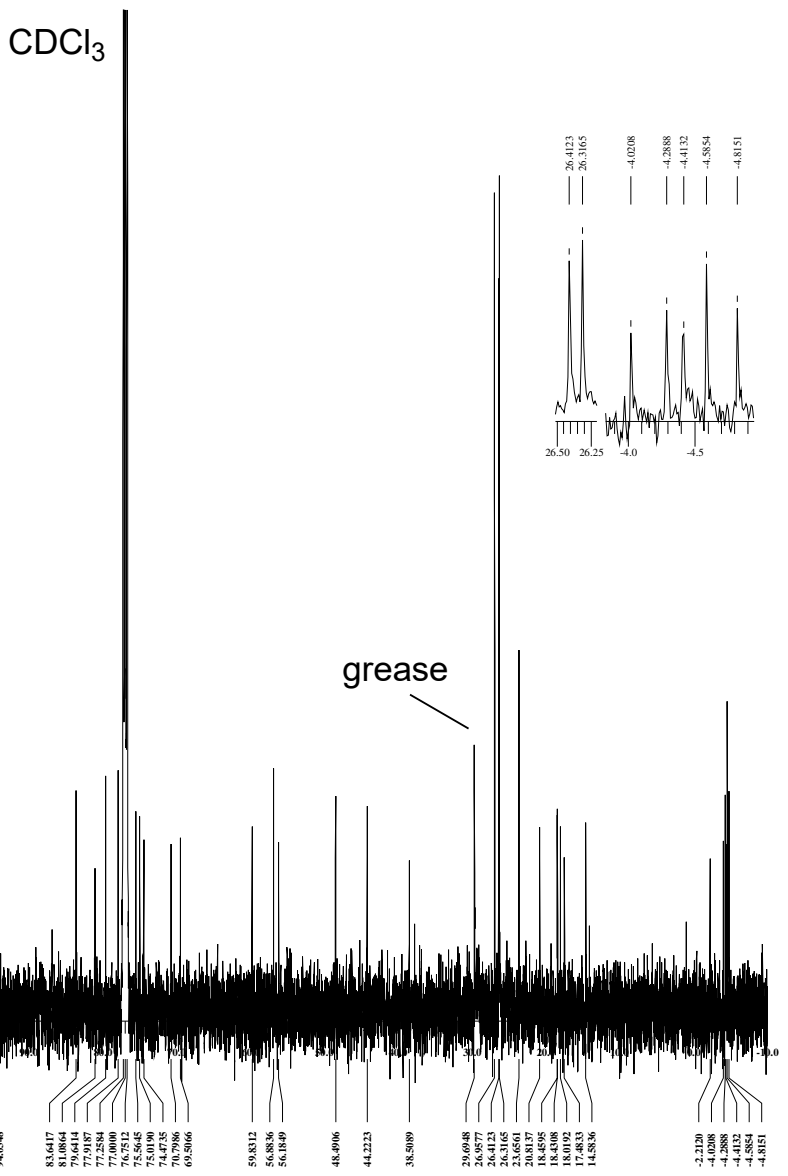




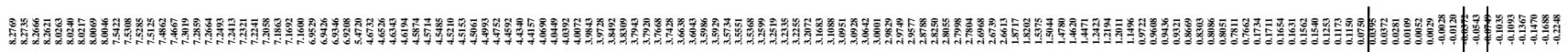

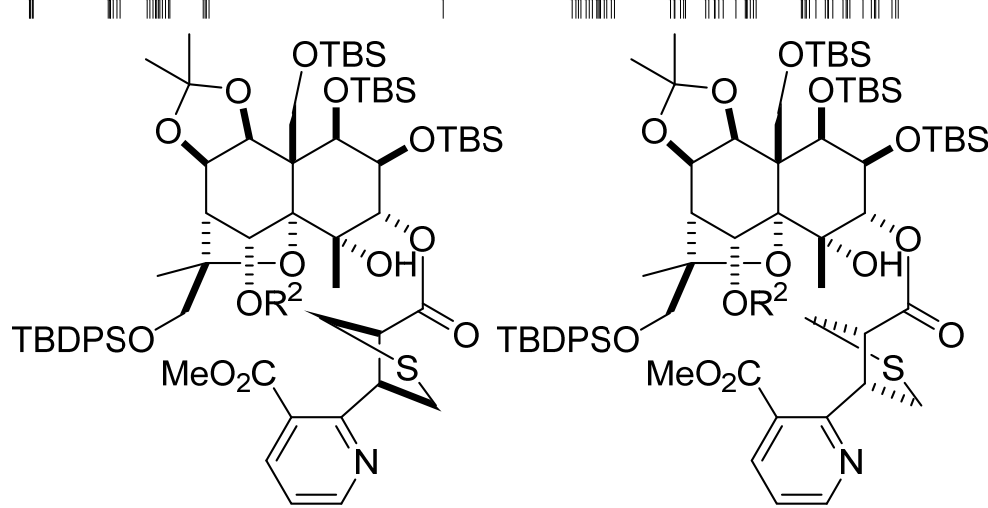

57: $\mathrm{R}^{2}=\mathrm{MOM}$

58: $R^{2}=M O M$

crude $\mathbf{5 7 / 5 8}=1: 3.2$

${ }^{1} \mathrm{H}$ NMR $\left(400 \mathrm{MHz}, \mathrm{CDCl}_{3}\right)$

$\mathrm{CHCl}_{3}$

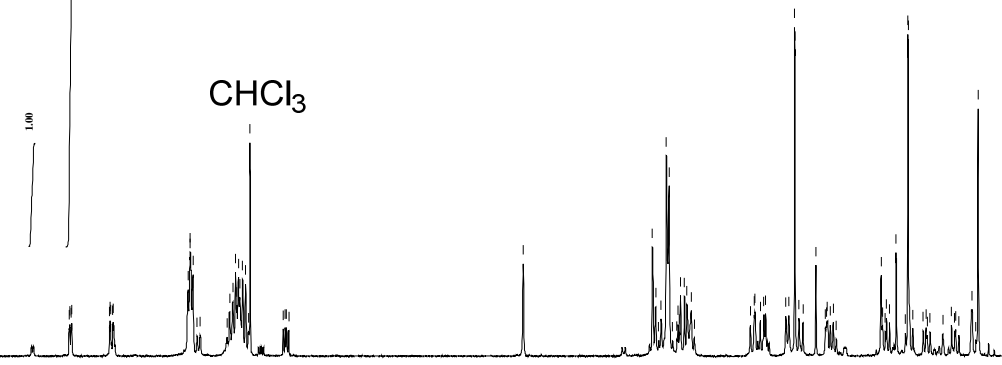

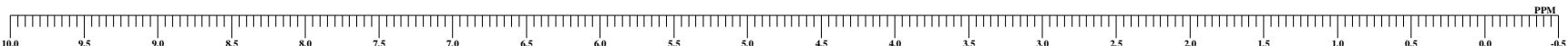




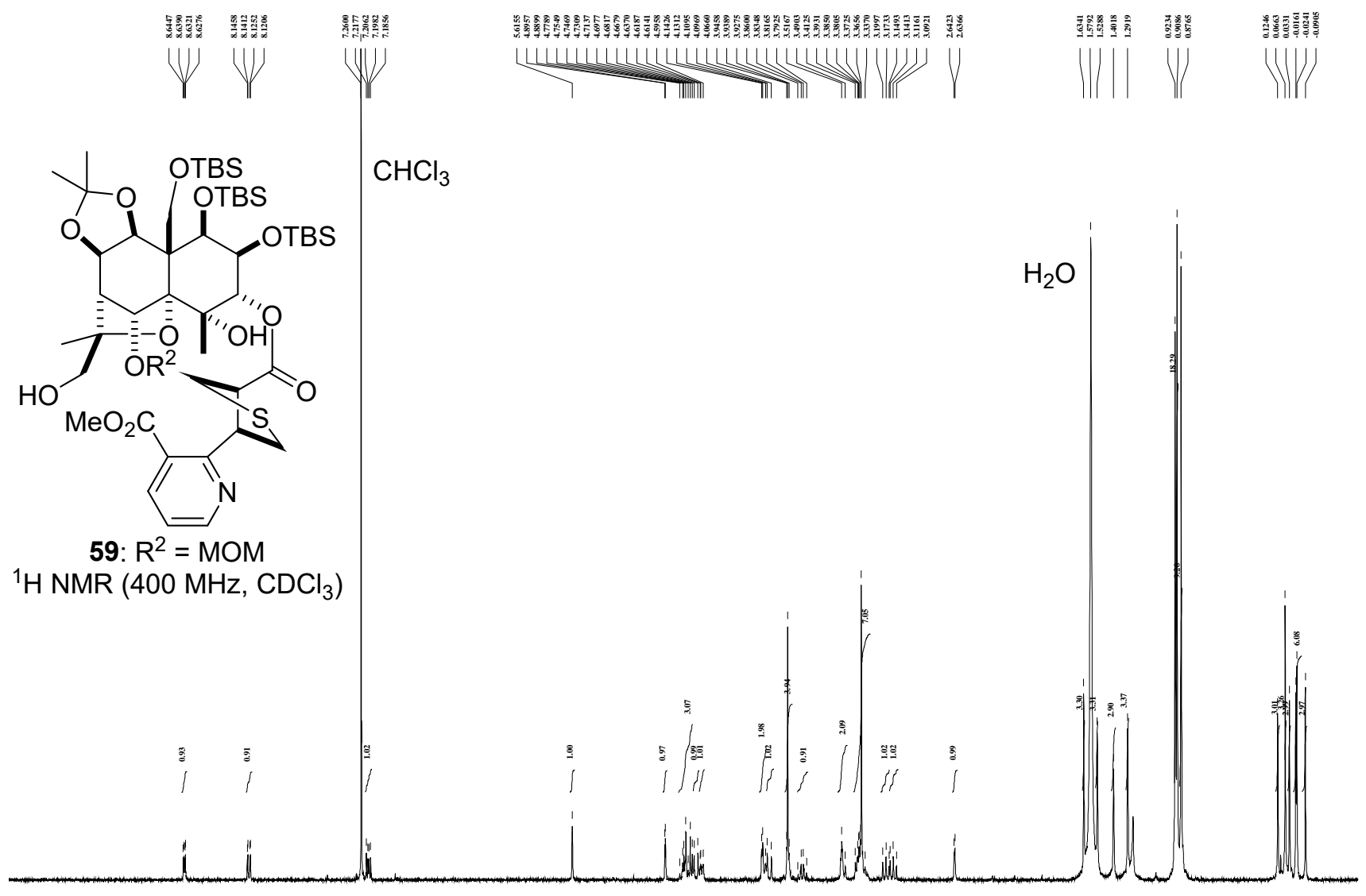

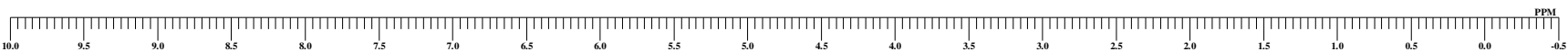
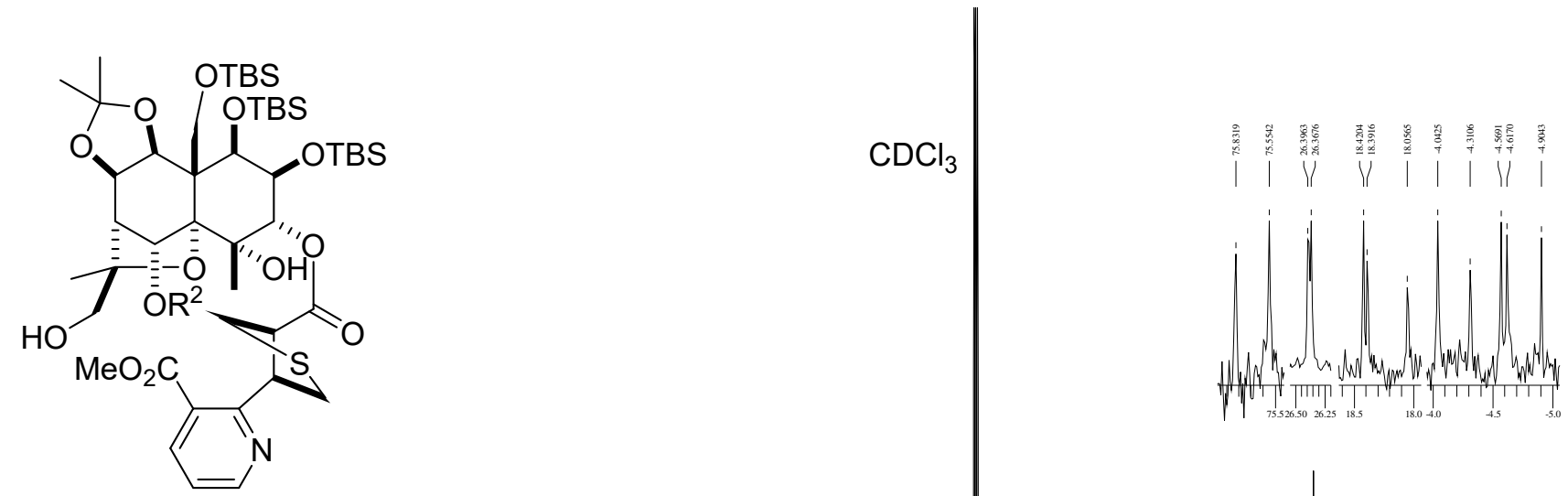

59: $R^{2}=$ MOM

${ }^{13} \mathrm{C}\left\{{ }^{1} \mathrm{H}\right\}$ NMR $\left(125 \mathrm{MHz}, \mathrm{CDCl}_{3}\right)$

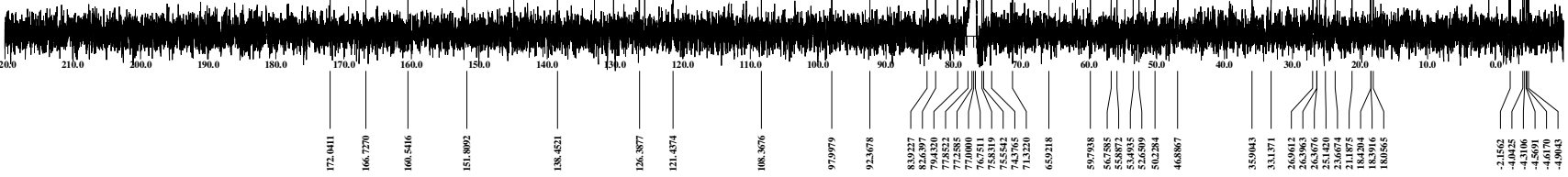




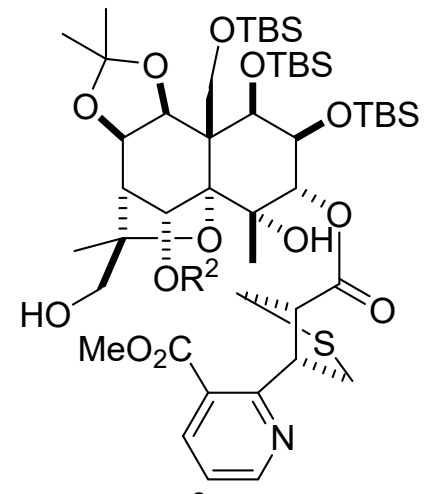

60: $\mathrm{R}^{2}=\mathrm{MOM}$

${ }^{1} \mathrm{H}$ NMR $\left(500 \mathrm{MHz}, \mathrm{CDCl}_{3}\right)$

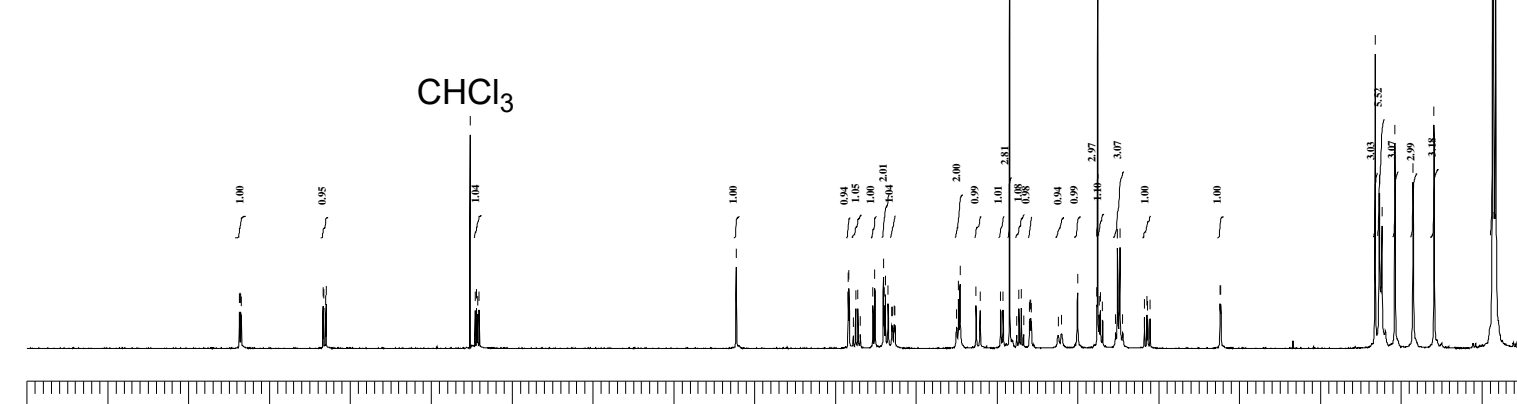

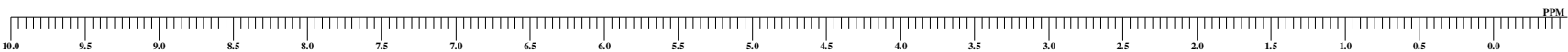

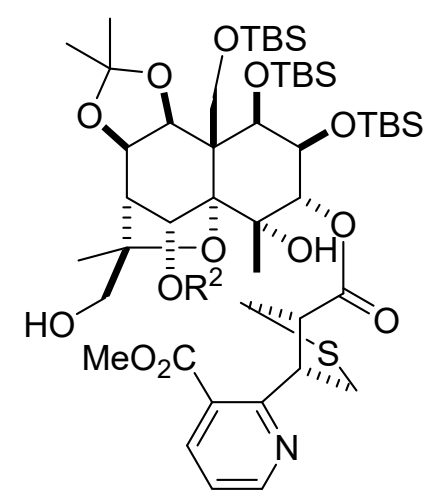

60: $\mathrm{R}^{2}=\mathrm{MOM}$

${ }^{13} \mathrm{C}\left\{{ }^{1} \mathrm{H}\right\} \mathrm{NMR}\left(125 \mathrm{MHz}, \mathrm{CDCl}_{3}\right)$
$\mathrm{CDCl}_{3}$
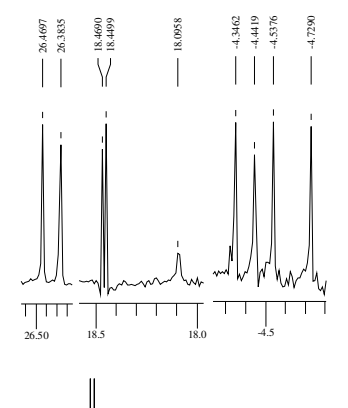

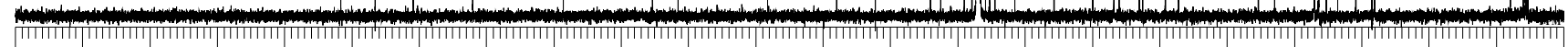

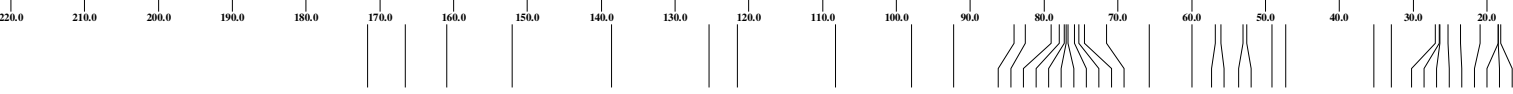




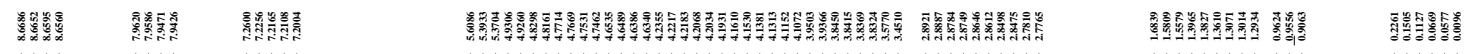

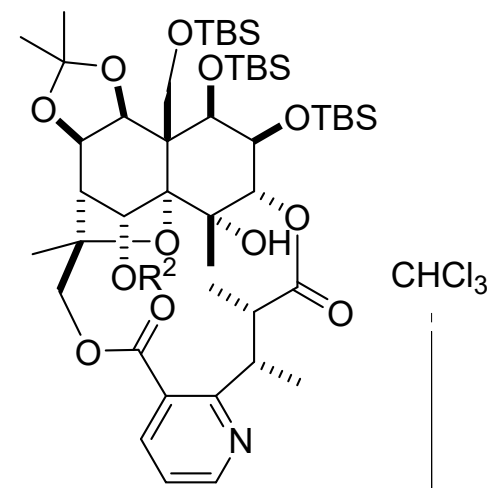

63: $\mathrm{R}^{2}=\mathrm{MOM}$

${ }^{1} \mathrm{H}$ NMR $\left(500 \mathrm{MHz}, \mathrm{CDCl}_{3}\right)$

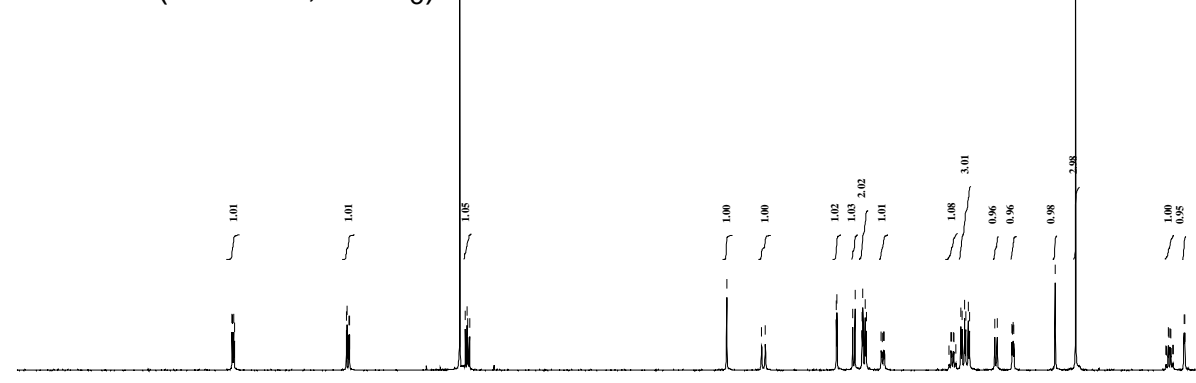

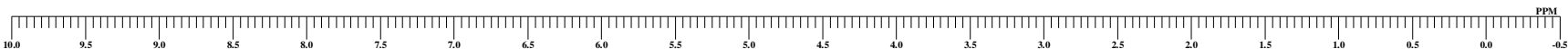
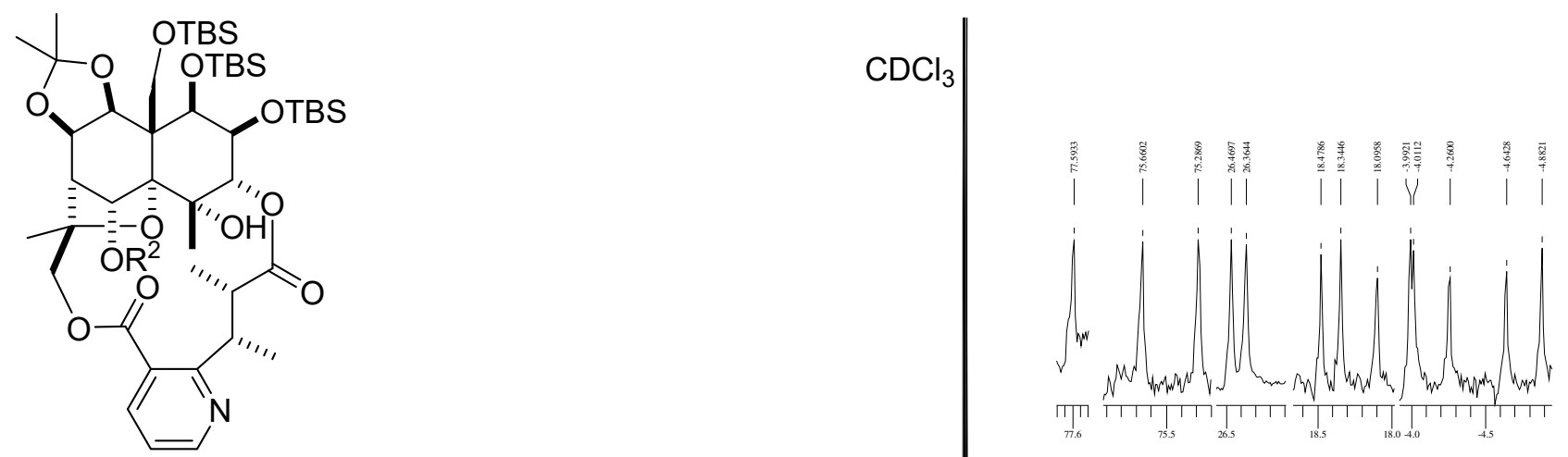

63: $\mathrm{R}^{2}=\mathrm{MOM}$

${ }^{13} \mathrm{C}\left\{{ }^{1} \mathrm{H}\right\}$ NMR $\left(125 \mathrm{MHz}, \mathrm{CDCl}_{3}\right)$
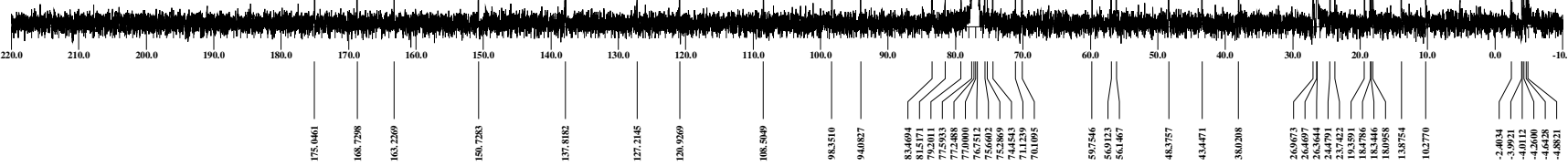


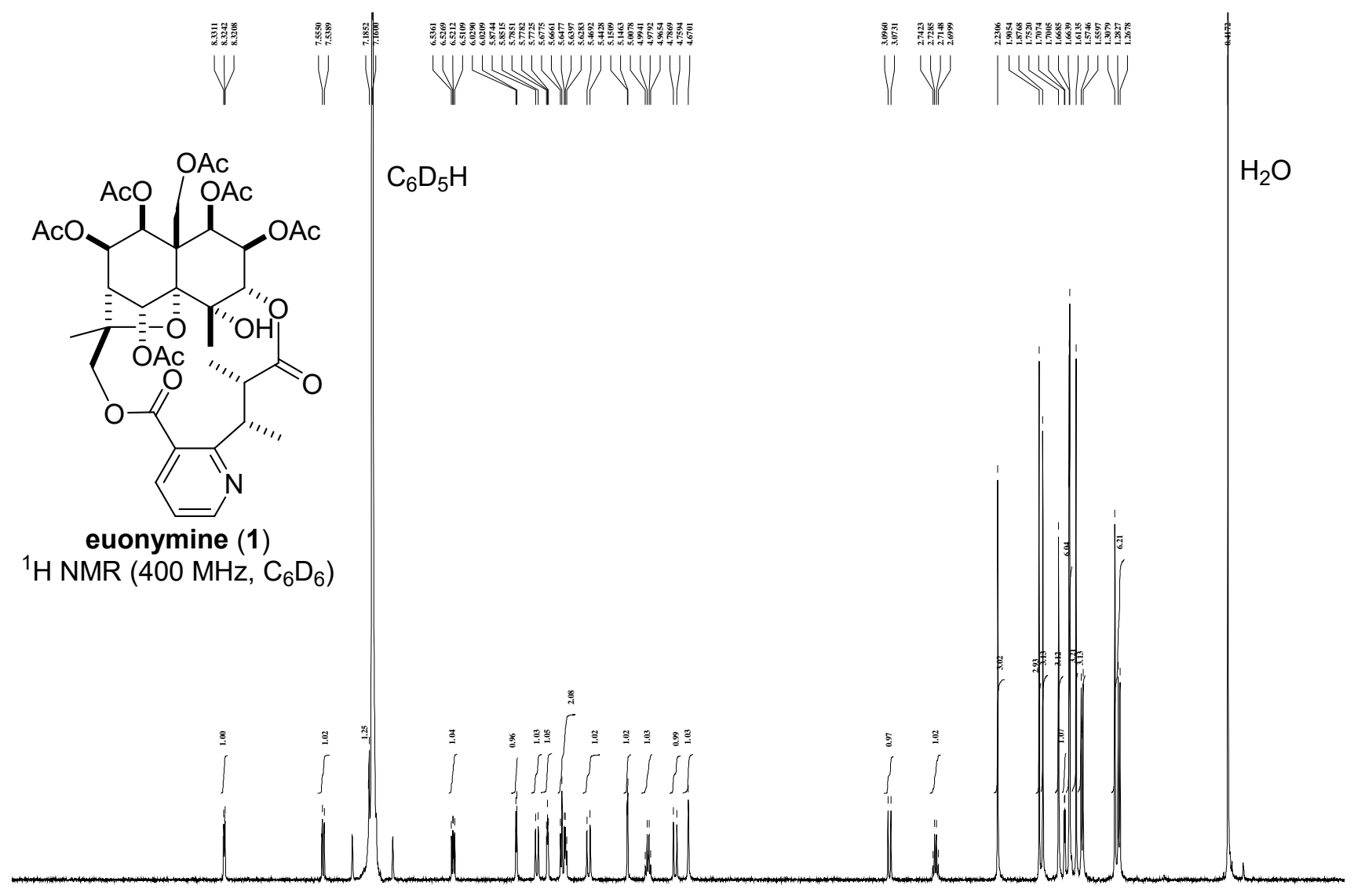

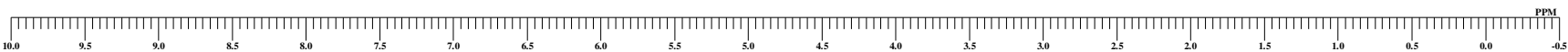

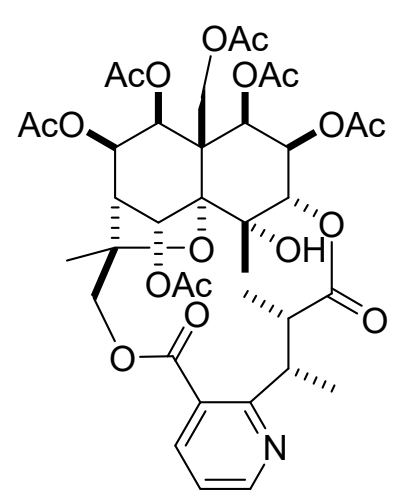

$\mathrm{C}_{6} \mathrm{D}_{6}$

euonymine (1)

${ }^{13} \mathrm{C}\left\{{ }^{1} \mathrm{H}\right\} \operatorname{NMR}\left(125 \mathrm{MHz}, \mathrm{C}_{6} \mathrm{D}_{6}\right)$

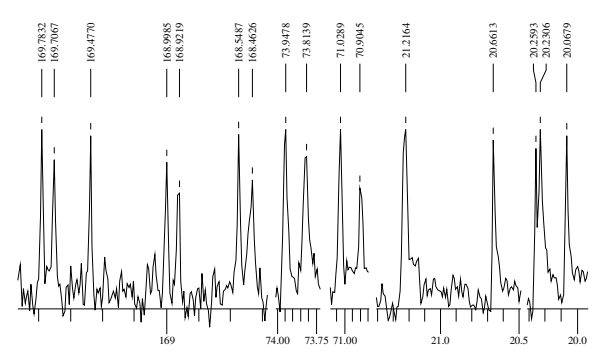




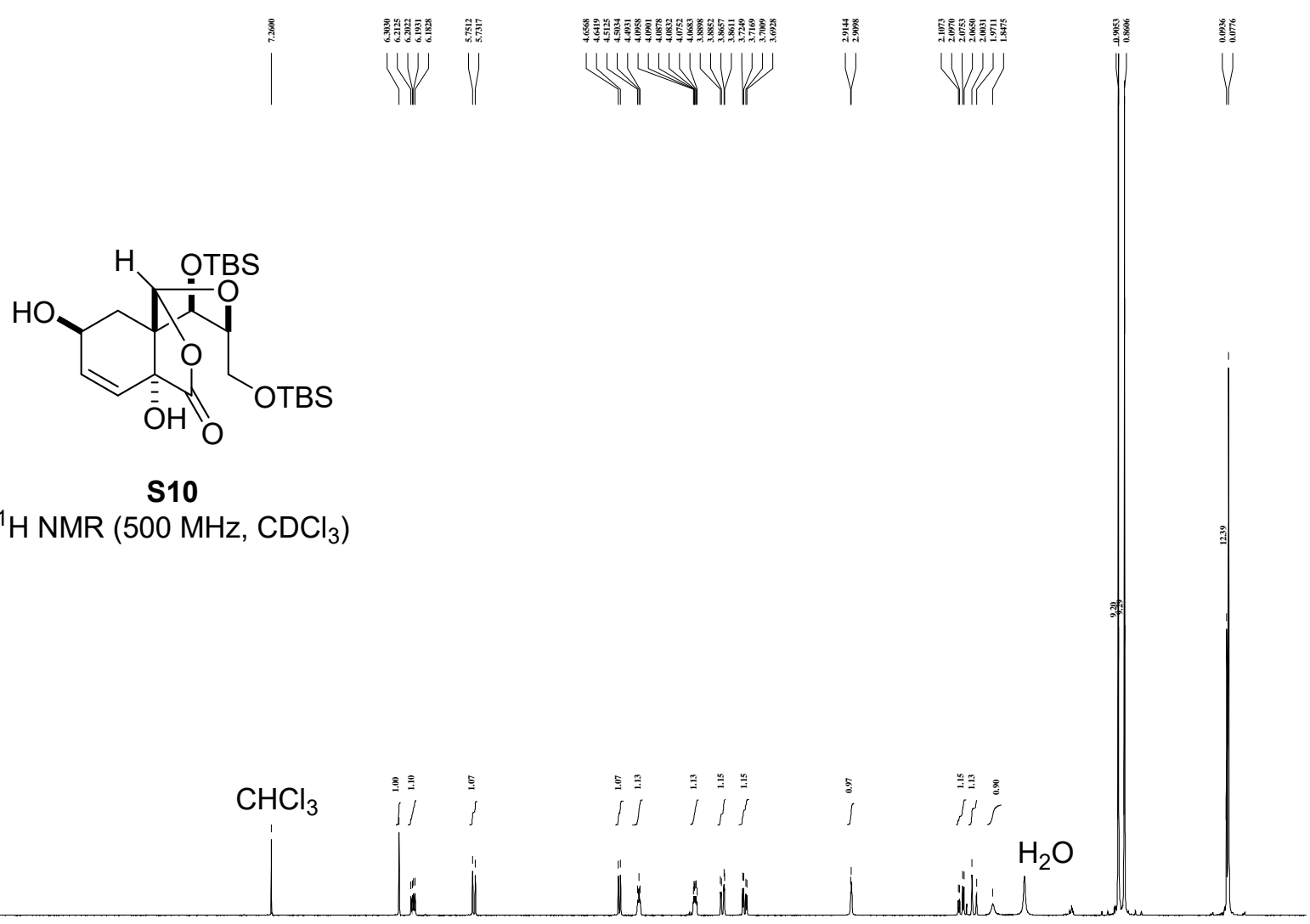

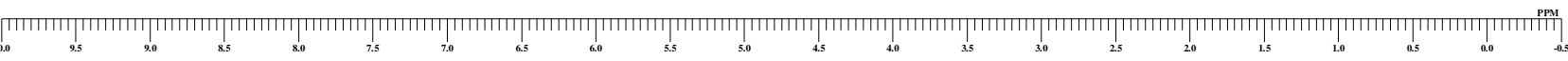

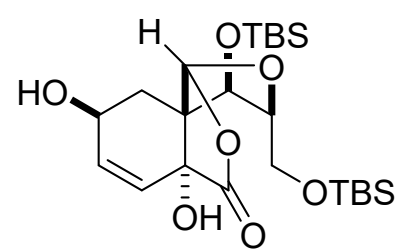

S10

${ }^{13} \mathrm{C}\left\{{ }^{1} \mathrm{H}\right\}$ NMR $\left(125 \mathrm{MHz}, \mathrm{CDCl}_{3}\right)$
$\mathrm{CDCl}_{3}$

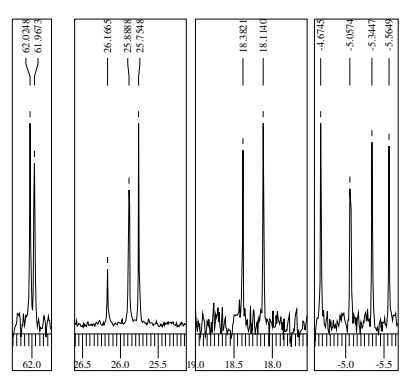




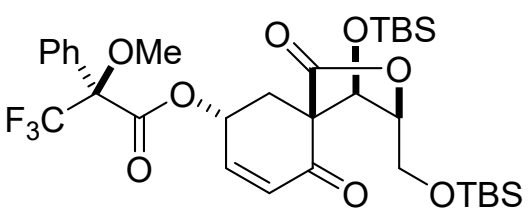

S11a

${ }^{1} \mathrm{H}$ NMR $\left(400 \mathrm{MHz}, \mathrm{CDCl}_{3}\right)$

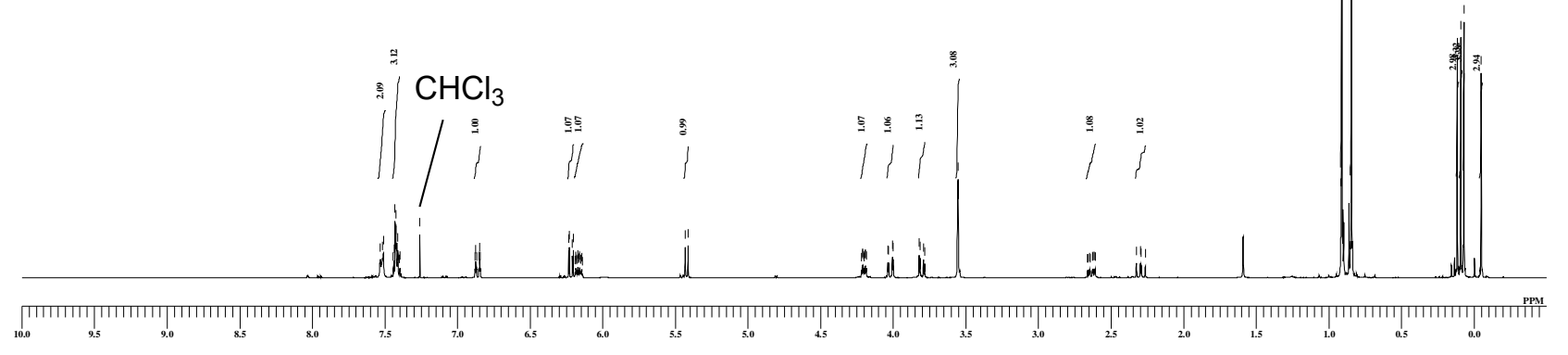

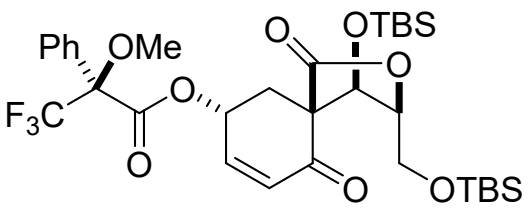

S11a

${ }^{13} \mathrm{C}\left\{{ }^{1} \mathrm{H}\right\} \operatorname{NMR}\left(125 \mathrm{MHz}, \mathrm{CDCl}_{3}\right)$

$\mathrm{CDCl}_{3}$

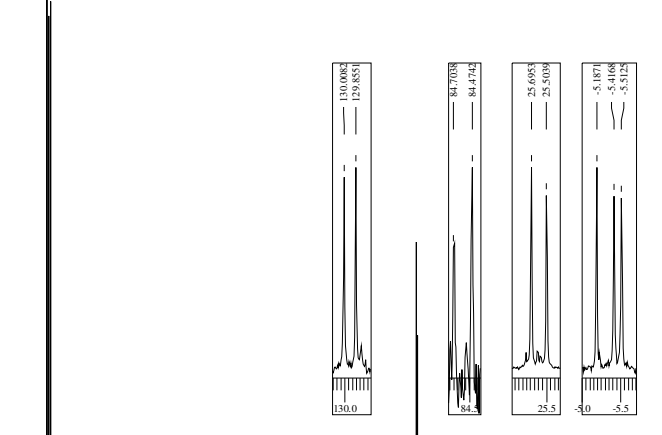

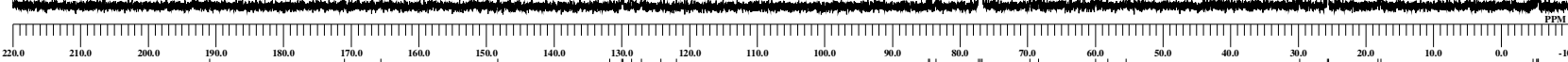




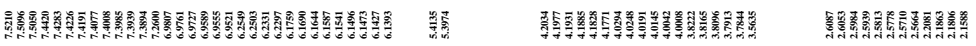
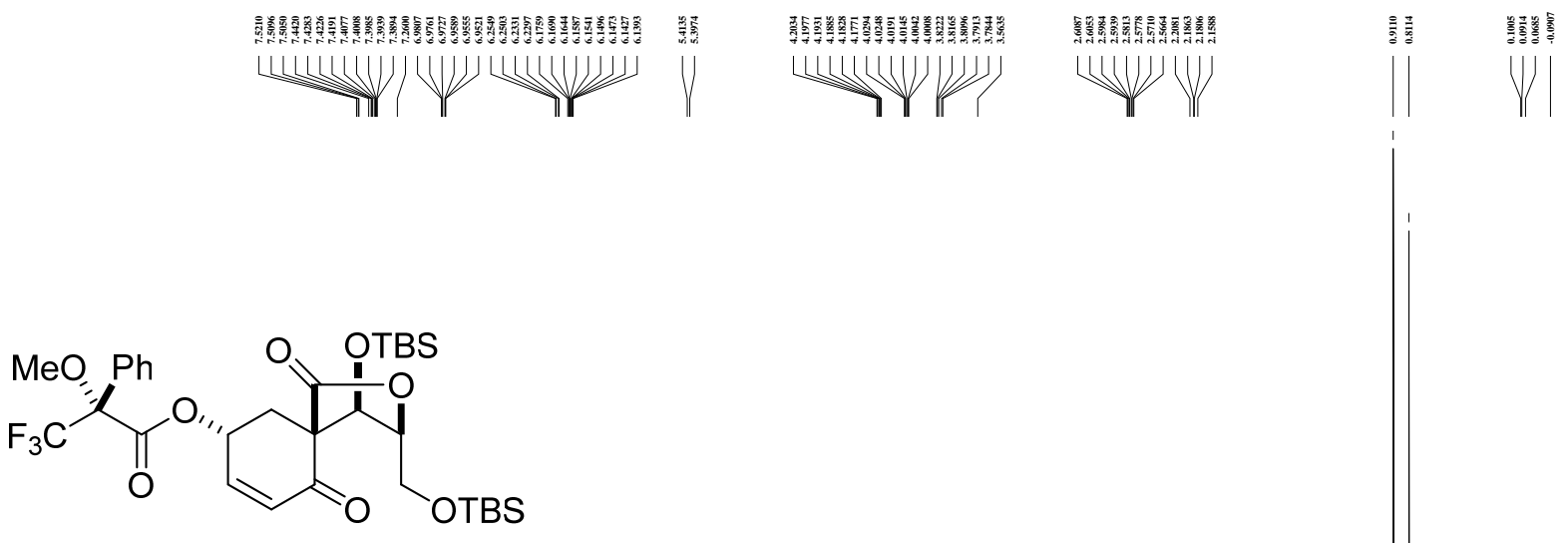

S11b

${ }^{1} \mathrm{H}$ NMR $\left(500 \mathrm{MHz}, \mathrm{CDCl}_{3}\right)$

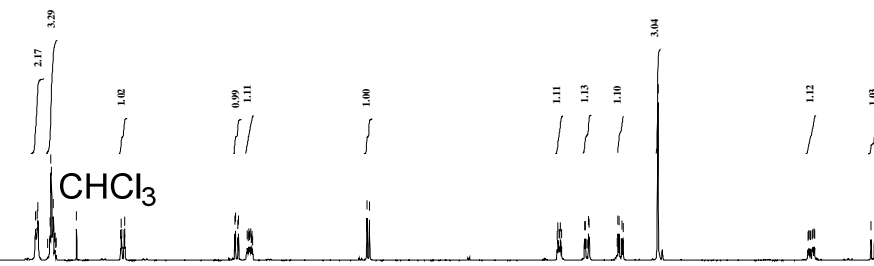

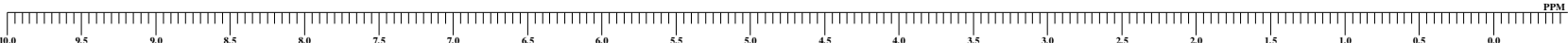

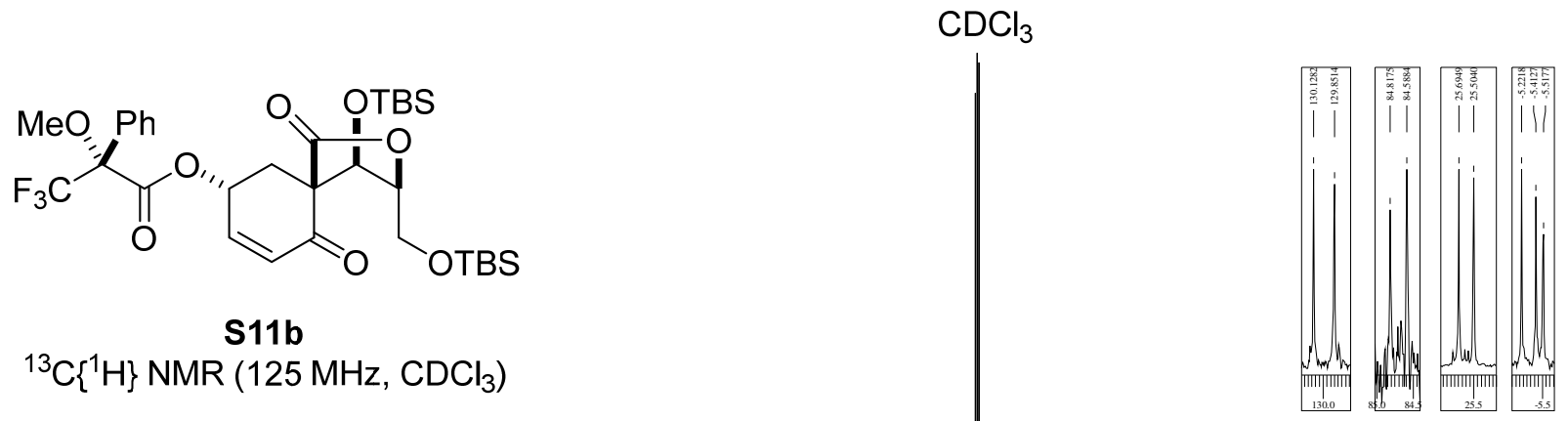

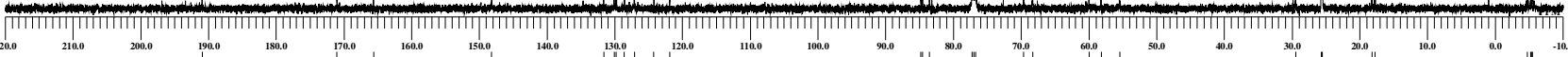

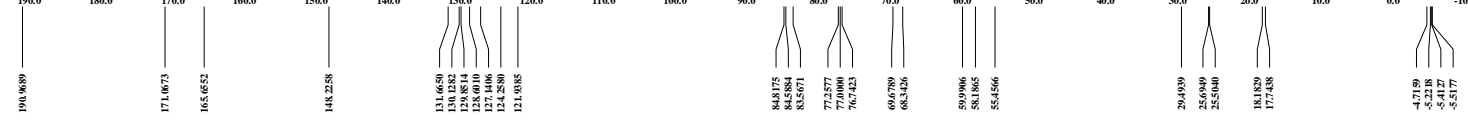

